
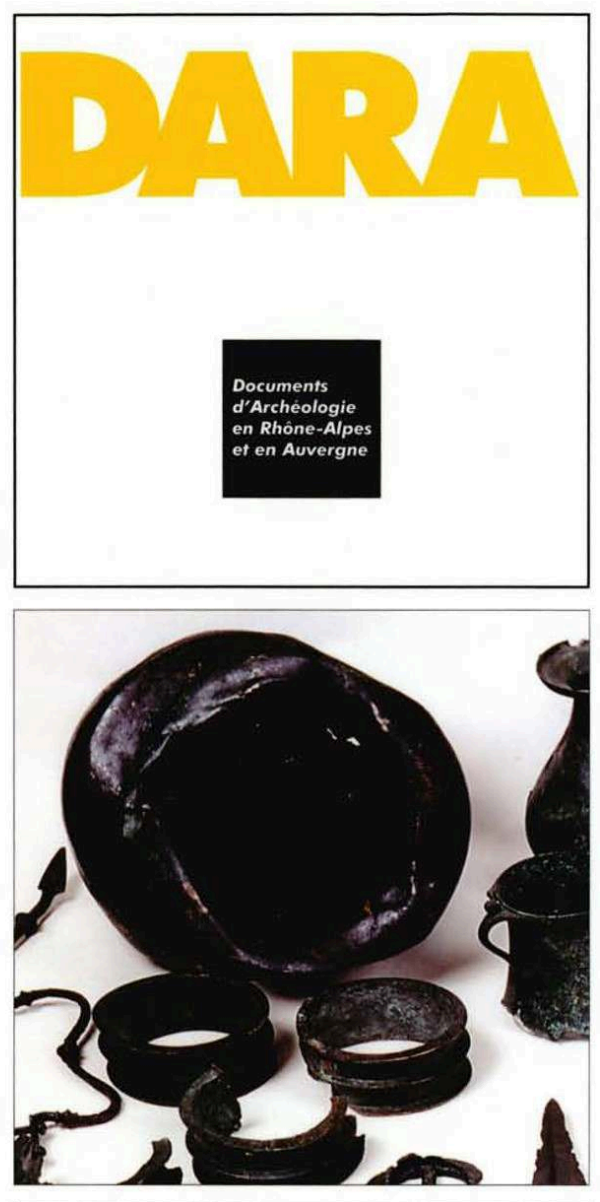

LA TOMBE À CHAR DE VERNA (ISÈRE) :

TÉMOIGNAGE

DE L'ARISTOCRATIE CELTIQUE EN TERRITOIRE ALLOBROGE

sous la direction de

Franck Perrin et Martin Schönfelder 


\section{La tombe à char de Verna (Isère)}

Témoignage de l'aristocratie celtique en territoire allobroge

\section{Franck Perrin et Martin Schönfelder (dir.)}

DOI : 10.4000/books.alpara.1921

Éditeur : Alpara

Lieu d'édition : Lyon

Année d'édition : 2003

Date de mise en ligne : 2 juin 2016

Collection : DARA

ISBN électronique : 9782916125404

\section{Qbooks}

http://books.openedition.org

Édition imprimée

ISBN : 9782951614543

Nombre de pages : 156

Référence électronique

PERRIN, Franck (dir.) ; SCHÖNFELDER, Martin (dir.). La tombe à char de Verna (Isère) : Témoignage de l'aristocratie celtique en territoire allobroge. Nouvelle édition [en ligne]. Lyon : Alpara, 2003 (généré le 12 janvier 2021). Disponible sur Internet : <http://books.openedition.org/alpara/1921>. ISBN : 9782916125404. DOI : https://doi.org/10.4000/books.alpara.1921.

Ce document a été généré automatiquement le 12 janvier 2021. II est issu d'une numérisation par reconnaissance optique de caractères.

(c) Alpara, 2003

Conditions d'utilisation:

http://www.openedition.org/6540 
En 1818 , à une trentaine de kilomètres à l'est de Lyon, fut fouillé près du château de Verna un tumulus qui livra un exceptionnel ensemble du début du I ${ }^{\text {er }}$ S. av. J.-C. (100 à 80) alors décrit par François Artaud: un char à quatre roues, des vases italiques en bronze, plusieurs panoplies militaires dont des épées volontairement pliées, ainsi qu'un bassin étrusque daté, pour sa part, du IV s. av. J.-C.

Cette collection appartient aujourd'hui à la commune de Hières-sur-Amby (Isère). À l'initiative de Franck Perrin, universitaire spécialiste de l'âge du Fer, elle est ici publiée près de deux siècles après sa découverte. Le mobilier métallique est étudié avec précision par Martin Schönfelder, du Römisch-Germanisches Zentralmuseum de Mayence. Des fragments brûlés d'os humains et animaux, des traces manifestes d'un "sacrifice", des objets permettent d'interpréter cet ensemble comme une sépulture fastueuse, probablement celle d'un aristocrate allobroge de haut rang qu'acompagnaient sur le bûcher funéraire ses biens les plus précieux, à l'image des cérémonies "magnifiques et somptueuses" décrites par Poséidonios d'Apamée, auteur grec contemporain de ces princes gaulois, qui trouvent ici leur plus spectaculaire illustration archéologique.

Das Wagengrab von Verna (Dép. Isère), einige Kilometer südwestlich der befestigten Höhensiedlung von Larina gelegen, ist eine der ältesten archäologischen Ausgrabungen in Frankreich. Der Grabhügel mit den verbrannten Beigaben wurde bereits 1818 ausgegraben und im selben Jahr durch François Artaud, den Archäologen und späteren Direktor des Museums von Lyon, in der Presse publiziert. Das Originalmanuskript und eine Reihe heute noch erhaltener Tafeln bilden eine bewundernswerte Dokumentation, einerseits aufgrund der Exaktheit der Befundbeobachtungen, andererseits aufgrund der Schlussfolgerungen, zu denen der Autor gelangte. Wenngleich die Erinnerung an diese Entdeckung von einigen Gelehrten weitergegeben wurde, ist es der Ankauf der Sammlung 1995 durch das Maison de patrimoine de Hières-surAmby gewesen, der es bewirkte, dass eine Restaurierung in den Werkstâtten des RömischGermanischen Zentralmuseums in Mainz und eine wissenschaftliche Aufarbeitung der Funde in die Wege geleitet wurde. Es handelt sich um einen der reichsten Fundkomplexe vom Ende der keltischen Epoche, aus der Zeit um 100 bis 80 v. Chr. Er beinhaltet unter anderem Schmuck, Waffen, zahlreiche Gefäße, vor allem aus Bronze, und einen Wagen. Dieses Fahrzeug, einer der seltenen Vierrädrer dieser Zeit, ist ein wirklicher, keltischer Prunkwagen mit bronzenen Nabenringen, bronzeverzierten Achsnägeln und einem mit Blechen und Ringen verzierten Wagenkasten ; in Teilbereichen belegt er trotzdem die Übernahme technischer Neuerungen aus der römischen Welt. Die Kriegerausstattungen sind ungewöhnlich reich : fünf Schwerter, sechs Lanzenspitzen, drei Schildbuckel; hinzufiigen muss man noch einen Helm, der in den Originalhandschriften erwähnt wurde, der aber heute fehlt. Die Mehrzahl der Bronzegefäße eine Kanne, Griffschalen, Becken, ein Becher und Situlen - wurde im Rahmen des Bestattungszeremoniells zerstört; es sind die republikanischen Gefäßtypen. die im Reinigungsritual und beim Trinken von Wein verwendet wurden. Sie fallen durch ihre besondere Größe auf, die die anderer Stücke übersteigt; u.a. daraus wird auf einen sozial sehr hochgestellten Status des Bestatteten geschlossen. Das Bronzegeschirr beinhaltet auch einzigartige und sehr seltene Gefäßtypen, darunter ein etruskisches Becken des 4. Jhs. v. Chr. wohl ein Familienerbstück, das ebenfalls mit verbrannt wurde. Diese Untersuchung mit all ihren Unsicherheiten, die der Bearbeitung von Altfunden anhaften, führt zur Hypothese, dass zu Beginn der Romanisierung ein Adeliger der Allobroger im Grenzgebiet seines Stammes einen Grabritus gewählt hat, der in der frühen Eisenzeit verankert ist; überdies liegt sein Bestattungsplatz inmitten eines Grabhügelfeldes vom Ende der Bronzezeit. 


\section{SOMMAIRE}

Les Auteurs

\section{Remerciements}

\section{Avant-propos \\ Jean Guibal}

\section{Chapitre 1. Historique de la découverte} Franck Perrin

La « collection de Verna »

Une sépulture mégalithique campaniforme et une tombe à char de la Tène finale La documentation écrite : publication et archives

Conclusions

\section{Chapitre 2. Le contexte de la découverte} Franck Perrin et Robert Royet

Les allobroges à la fin de l'âge du Fer

Choix de textes antiques sur les allobroges (fin IIe-milieu Ier s. av. J.-C.)

L’Isle Crémieu : géographie et données archéologiques

\section{Chapitre 3. Le dépôt funéraire}

OSSEMENTS HUMAINS ET ANIMAUX. Fr. Blaizot, D. Lalaï

L'ÉTAT DE CONSERVATION DU MOBILIER MÉTALLIQUE. B. Herbold

LES PARURES VESTIMENTAIRES. J.-P. Guillaumet, M. Schönfelder

LA VAISSELLE EN BRONZE. M. Schönfelder, avec une contribution de A.-M. Adam LES PANOPLIES GUERRIÈRES. M. Schönfelder avec une contribution de B. Herbold

LE CHAR. M. Schönfelder

LES ÉLÉMENTS DU HARNACHEMENT. M. Schönfelder

L'OUTILLAGE. J.-P. Guillaumet, M. Schönfelder LES OBJETS MÉTALLIQUES DIVERS. M. Schönfelder

LE MOBILIER CÉRAMIQUE. V. Guichard, F. Olmer

\section{Synthèse}

La cohérence des données archéologiques

Interprétation du dépôt à la lumière des connaissances modernes : sépulture ou dépôt ?

Sélection et traitement du mobilier déposé dans la tombe

Datation de la tombe

La tombe de Verna parmi les sépultures aristocratiques de la fin de l'âge du Fer

Annexes

Catherine Bellon et Stéphane Carrara

Bibliographie

Crédits illustrations 


\section{Les Auteurs}

A.-M. Adam : Professeur, Université Marc-Bloch, Strasbourg.

C. Bellon : INRAP Rhône-Alpes/Auvergne.

F. Blaizot : Anthropologue, INRAP Rhône-Alpes/Auvergne.

4 S. Carrara : Doctorant Université Lyon 2.

5 V. Guichard: Directeur général, Centre Archéologique Européen du Mont Beuvray.

6 J.-P. Guillaumet : Directeur de Recherche CNRS, UMR 5594, Dijon.

7 B. Herbold : Römisch-Germanisches Zentralmuseum, Mainz.

8 D. Lalaï : Archéozoologue, INRAP Rhône-Alpes/Auvergne.

9 F. Olmer : Chercheur CNRS, UMR 5594, Dijon.

10 F. Perrin : Maître de Conférences, Université Lyon 2, UMR 5138 Lyon.

11 R. Royet : Conservateur, Service Régional de l'Archéologie, Lyon.

12 M. Schönfelder: Chercheur associé UMR 5594, Dijon; Römisch-Germanisches Zentralmuseum, Mainz. 
Remerciements 
1 Nous tenons à remercier ici MM. Jean-Pascal Jospin et José Dias, respectivement Conservateur au Musée dauphinois de Grenoble et Directeur de la Maison du Patrimoine de Hières-sur-Amby pour l'intérêt qu'ils ont bien voulu porter à l'étude de la collection de Vema; ils ont su, l'un et l'autre, faciliter l'accès au mobilier archéologique et par là-même son étude.

2 Nos remerciements également à M. Ulrich Schaaff, ancien directeur du département protohistorique du Römisch-Germanisches Zentralmuseum de Mayence et à son équipe pour la restauration des objets métalliques et pour leur accueil et soutien.

3 L'étude sur le mobilier métallique a été réalisée par l'un d'entre nous grâce à une bourse post-doctorale du Conseil Régional de Bourgogne en 2000/2001. L'équipe, qui a assuré un accueil très chaleureux et très motivant pendant une année, était celle de Jean-Paul Guillaumet (UMR 5594/3), basée au Centre Archéologique Européen du Mont Beuvray. Un grande merci à celui-ci et à tous les étudiants et étudiants-chercheurs, qui nous ont soutenu, aidé et inspiré. C'est également au CAE du Mont Beuvray, et grâce à son directeur Vincent Guichard, que l'illustration photographique de ce volume a été en grande partie réalisée.

4 L'illustration graphique quant à elle a pu être effectuée par Marion Baudrand au Musée de la Civilisation Gallo-Romaine de Lyon, grâce à l'amabilité des Conservateurs Jacques Lasfargues et Hugues Savay-Guerraz.

5 Nous exprimons toute notre gratitude à M. et Mme Huon de Kermadec pour nous avoir confié les manuscrits originaux des archives en leur possession.

6 Gérard Bruyères, Conservateur des Archives Municipales de la ville de Lyon a su, par sa connaissance de François Artaud, témoigner de son attention pour cette étude, alors que la consultation des diverses archives a été effectuée à l'Académie des Sciences Belles Lettres et Arts de Lyon.

7 Plus largement, nous exprimons notre reconnaissance à l'ensemble des collègues qui ont su faire don de leur temps et nous apporter leur aide grâce à leurs connaissances ou à leur soutien technique, avec une mention spéciale à Catherine Bellon pour sa relecture du manuscrit, notamment pour les chapitres écrits dans "le français d'un étranger", et pour avoir assuré la coordination du volume avec le secrétariat des DARA. 


\title{
Avant-propos
}

\author{
Jean Guibal
}

1- La plaine où a été mise au jour la tombe à char de Verna, au pied du plateau de l'Isle Crémieu

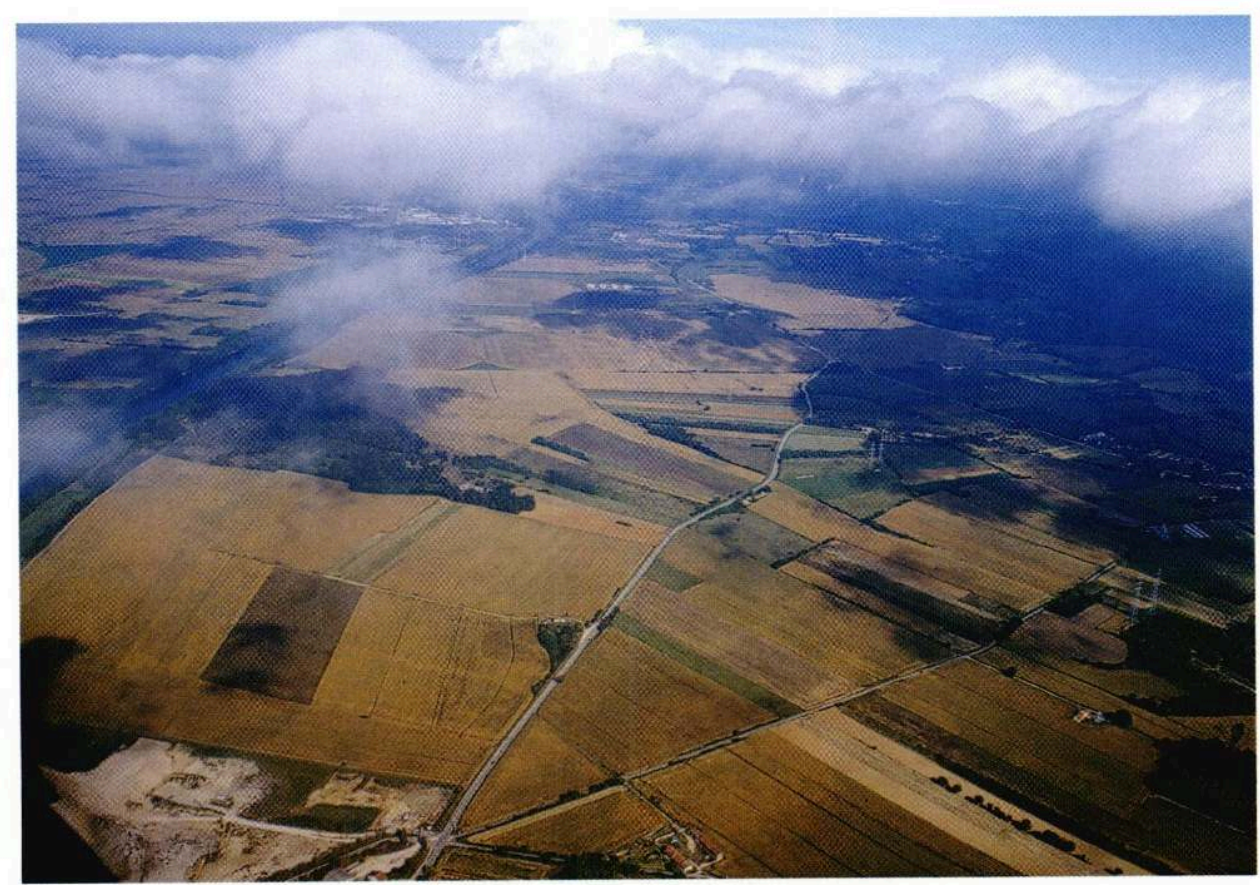

1 Au moment où l'on s'interroge sur le devenir de l'archéologie, au moment où l'on tente quelques timides expériences de décentralisation dans le domaine de la gestion du patrimoine, cet ouvrage vient consacrer de précieuses et exemplaires collaborations. Un musée municipal, la Maison du Patrimoine de Hières-sur-Amby, a littéralement sauvé le mobilier de la tombe celte de Verna, promis à la dispersion lors d'une vente à Drouot en 1995: que son conservateur d'alors, Patrick Porte, et la maire de la commune $^{1}$, Madame Dominique Blanc, en soient vivement remerciés. Il a fallu ensuite attendre plusieurs années pour trouver une solution face à la très onéreuse opération de restauration que réclamait la centaine d'objets métalliques qui composent ce 
mobilier. La générosité du laboratoire de restauration du Romisch-Germanisches Zentralmuseum de Mayence (Allemagne) a permis cette intervention : nous exprimons notre gratitude au docteur Ulrich Schaaff, alors directeur du laboratoire, et à Martin Schönfelder, spécialiste des tombes à char, qui a bien voulu conduire cette restauration et co-diriger la présente publication. Collaboration également de Jean-Paul Guillaumet, chercheur au CNRS (Archéologie, cultures et sociétés en Bourgogne et en France orientale) qui a rendu possible l'accueil de Martin Schönfelder et l'acquisition d'une bourse délivrée par le conseil régional de Bourgogne. Et enfin, celle d'un enseignantchercheur de l'Université Lumière-Lyon 2, Franck Perrin, qui a accepté de conduire la recherche sur cet ensemble et son contexte. Mais il fallait encore l'engagement d'une association spécialisée dans ce type de publications archéologiques (l'Alpara), le soutien du Service régional de l'archéologie (relevant de la Drac Rhône-Alpes, ministère de la Culture) et celui de la Conservation du Patrimoine de l'Isère (service du Conseil général) pour que la publication voit le jour dans les meilleures conditions.

2 Malgré son caractère exceptionnel, la tombe de Verna était tombée dans l'oubli. Découvert en 1818 par la famille Dauphin, le mobilier qui la composait avait été conservé dans la bibliothèque du château familial. Seul François Artaud, conservateur chargé de l'archéologie au Musée des Beaux-Arts de Lyon, fit dès la découverte une première et unique description des objets. Le manuscrit de son rapport fut heureusement conservé par la famille de Verna qui a bien voulu nous autoriser à le reproduire et permettre ainsi au lecteur de mesurer la qualité et la pertinence de ses analyses.

3 La protohistoire de cette région du nord de l'Isère (fig. 1), délimitée par une large boucle du Rhône, s'enrichit ainsi d'une nouvelle somme de connaissances. Après l'ouvrage consacré, dans la même collection, à l'énigmatique dépôt cultuel du Camp de Larina étudié par le même Franck Perrin ; après la fastueuse tombe du Ville s. av. J.-C., fouillée en 1987 par Jean-Paul Guillaumet et Stéphane Verger; ou encore le véritable palais rural de Saint-Romain-de-Jalionas dont les origines gauloises sont désormais attestées par les recherches en cours de Robert Royet, le savoir accumulé par l'archéologie régionale s'avère prestigieux. En témoignent les collections des musées, mais tout autant la production éditoriale, complément indissociable de la conservation, et notamment celle que permet la présente collection.

4 Le caractère exceptionnel du mobilier de la tombe de Verna a déjà justifié l'exposition de quelques éléments au Musée de la Civilisation celtique du Mont-Beuvray, en 1999 (Les tombes des derniers aristocrates celtes), sous la direction de Vincent Guichard et Franck Perrin; sa présentation complète a eu pour cadre la grande exposition consacrée aux Allobroges, au Musée dauphinois de Grenoble, en 2003, conduite sous la direction de Jean-Pascal Jospin. Et l'ensemble du mobilier retournera par la suite, pour exposition permanente, à la Maison du Patrimoine de Hières-sur-Amby, dont la muséographie et l'ensemble de l'aménagement intérieur doivent être rénovés.

5 Loin d'être une fin en soi, les projets culturels présentent aussi pour intérêt de permettre aux chercheurs de réaliser de nouvelles investigations et de plus larges synthèses; et surtout engagent les institutions culturelles à les publier. C'est donc avec plaisir que la Conservation du Patrimoine de l'Isère s'est associée à cette édition, qui vient compléter et prolonger l'exposition du Musée dauphinois et la publication qui l'accompagne. Et qu'à ce titre, je suis heureux d'en remercier tous les collaborateurs, avec lesquels il faut désormais tenter d'approcher et de comprendre ce personnage et 
la culture à laquelle il appartenait, ce "prince » inhumé avec faste dans la plaine du Rhône, il y a plus de deux mille ans...

\section{NOTES DE FIN}

1. La commune de Verna s'écrit avec ou sans (s), la collection de Verna s'écrit sans (s) en référence à l'ancien propriétaire du site, le comte de Verna.

\section{AUTEUR}

JEAN GUIBAL

Directeur de la Conservation du Patrimoine de l'Isère 


\section{Chapitre 1. Historique de la découverte}

\section{Franck Perrin}

2- Vues des tumulus et de la sépulture mégalithique, planche IV de la collection privée

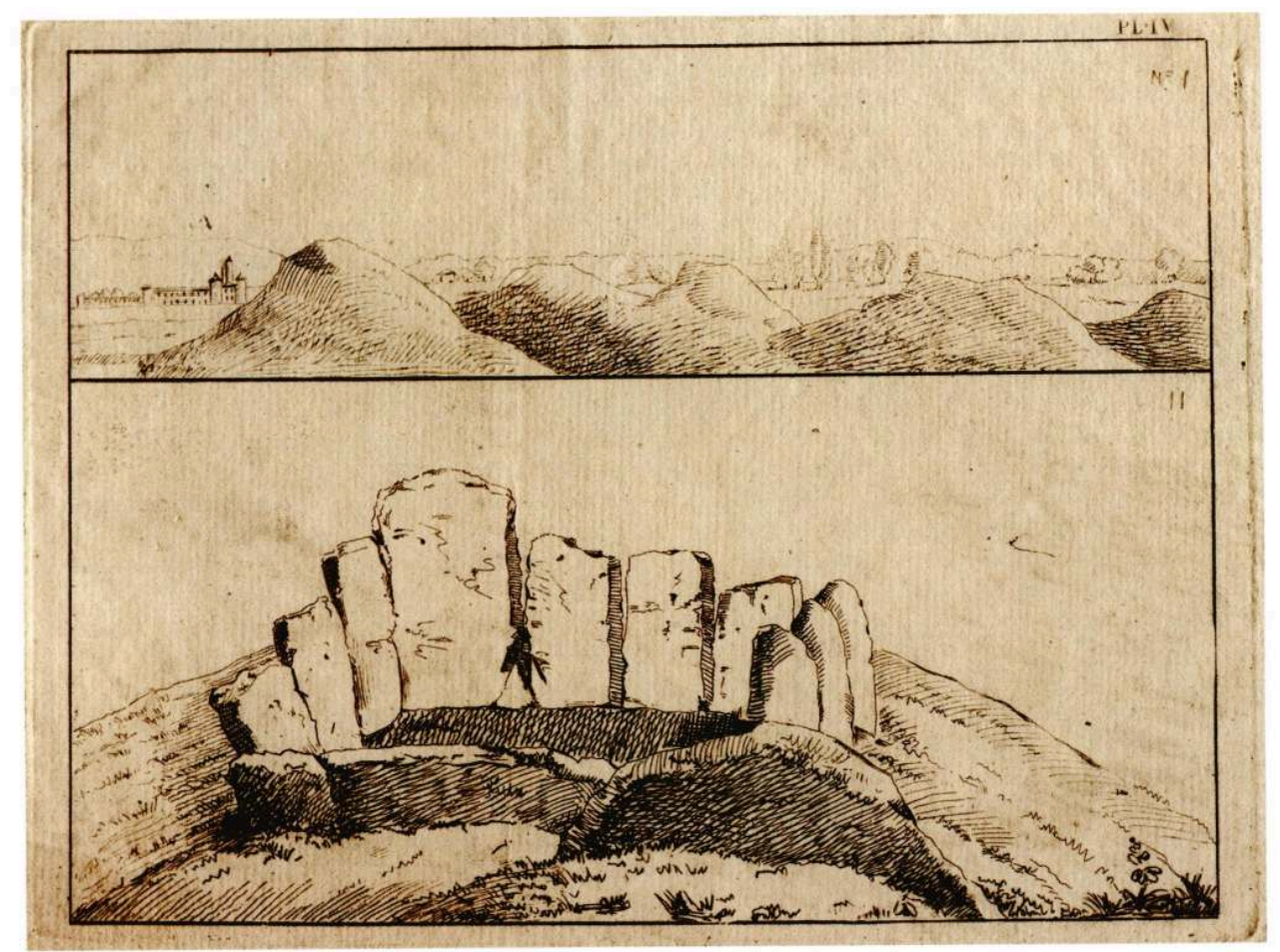

\section{La « collection de Verna »}

En 1995, une collection composée d'une centaine d'objets archéologiques fut mise en vente par un particulier à l'Hôtel Drouot à Paris et acquise par la Maison du Patrimoine de Hières-sur-Amby (Isère) qui en connaissait l'origine. En effet, quelques années 
auparavant, en 1986, ce mobilier avait été prêté par les anciens propriétaires (la famille de Verna) à la Maison du Patrimoine pour être photographié ; à cette occasion, un lot d'archives manuscrites et de gravures fut partiellement photocopié et cliché, puis transmis à différents chercheurs (J.-P. Guillaumet, S. Verger, F. Perrin) travaillant sur la protohistoire de l'Isle Crémieu. En 1997, sur nos recommandations, le mobilier entra au Laboratoire du Romisch-Germanisches Zentralmuseum de Mayence (Allemagne), pour y être restauré et étudié sous la direction du Dr. U. Schaaff.

2 Cette collection privée, que nous appellerons par commodité "collection de Verna ", était connue depuis longtemps, dès le début du XIXe s. en fait, époque à laquelle elle était apparue dans la littérature archéologique. Plusieurs chercheurs avaient eu le privilège de l'examiner, envisageant même de la publier: Ernest Chantre, alors directeur du musée d'Histoire naturelle de Lyon, Gabriel Chapotat, chercheur au CNRS, Aimé Bocquet, directeur du Centre de Préhistoire et de Documentation Alpine de Grenoble... Dès les années 1970, G. Chapotat avait mentionné l'existence de cette tombe à char d'un type nettement différent de celui de La Côte-Saint-André, alors en cours d'étude; le chercheur avait eu l'opportunité d'examiner le mobilier et connaissait, semble-t-il, l'emplacement de la nécropole ${ }^{1}$; en 1986, lors du colloque de l'AFEAF de Yenne-Chambéry, P. Porte, conservateur au service régional de l'Archéologie de RhôneAlpes et J.-P. Guillaumet, chercheur au CNRS, présentèrent une brève communication non publiée ${ }^{2}$ (Guillaumet, Porte 1986) sur la collection de Verna interprétée comme une tombe à char de La Tène finale découverte au début du XIXe s., puis peu à peu oubliée de la communauté scientifique, malgré quelques mentions dans le célèbre Manuel de Joseph Déchelette.

\section{Une sépulture mégalithique campaniforme et une tombe à char de la Tène finale}

3 Afin de faciliter l'exposé de cette découverte, il faut, avant toute chose, en préciser le contenu et la nature. En effet, comme toute collection privée, celle de la famille de Verna était susceptible de rassembler des objets de provenances variées, des pièces trouvées localement, mais aussi des acquisitions extra-régionales. Néanmoins, la collection comprenait deux ensembles archéologiques de provenance locale.

\section{Une sépulture mégalithique campaniforme réutilisée}

On doit à Aimé Bocquet d'avoir le premier observé que la collection de Verna regroupait deux lots d'objets chronologiquement distincts, mais découverts à peu de distance l'un de l'autre. En 1970, lors d'une visite au château de Verna, alors en la possession de M. Gailly de Taurine, il identifia un poignard chalcolithique et plusieurs tessons d'un vase campaniforme. L'examen des dessins et des annotations conservés à l'époque avec les objets apporta les précisons suivantes: le poignard avait été trouvé "dans le tombeau germain "; quant aux tessons contemporains, ils figuraient dans une restitution graphique sous la légende "trouvés au Mas du Charnier ou des Trois Pierres ». Cette localisation permit, par la suite, de situer près du lieu-dit "Le Charnier $\|^{3}$ les vestiges de ce qui s'avère une sépulture mégalithique réutilisée à la fin de l'âge du Bronze (Bronze Final IIb). Ces travaux furent publiés en 1976 (Bocquet 1976: 23-32), illustrés par une gravure provenant des archives de la famille de Verna, figurant une 
illustration ou une reconstitution de la sépulture mégalithique. Cette tombe mégalithique (fig. 2) avait fait l'objet d'une communication le 14 juin 1825, devant l'Académie des Sciences, Belles Lettres et Arts de Lyon, par François Artaud alors directeur du musée et de l'école royale des Arts de Lyon. Lors de cette intervention concernant les mégalithiques de Carnac, Fr. Artaud précisa avoir

« eu l'occasion d'examiner des monuments analogues au château de M. de Verna,

dans le département de l'Isère »:

le texte figure dans les archives de l'Académie des Sciences, Belles Lettres et Arts de Lyon ${ }^{4}$. Dans son ouvrage posthume Lyon souterrain, publié en 1846, l'auteur notait à propos des tumuli des environs du château de Verna que

«A une distance assez rapprochée (du dolmen) nous avons distingué une sépulture circulaire formée de pierres debouts, dans le genre de celles de Stonehenge, que l'on attribue à des Germains ou plutôt des Saxons. Celles de Verna qui, à notre persuasion, ont eu la curiosité de la fouiller (sic), y ont trouvé des vases grossiers, des squelettes rangés les uns à côté des autres, recouverts par de grandes briques (sic) inclinées... ».

Pour être complet, il faut signaler la présence d'un bracelet datable du Bronze final; compte-tenu des résultats des travaux d'A. Bocquet, il faut attribuer cet objet à une tombe secondaire de la sépulture mégalithique. Ainsi, le dolmen de Verna fut fouillé avant 1836, année de la mort de Fr. Artaud. En 1894, cette découverte fut mentionnée par François Gabut, sous le nom du «tombeau du Général Romain », une appellation née, selon ses dires, d'une tradition locale narrant le siège, par César en personne, du Camp de Larina voisin. A son époque, le dolmen était situé entre deux fermes appartenant au comte de Verna. Fouillé en 1972 par A. Bocquet, le monument est parfaitement localisé à proximité du croisement des routes D65 et D65b (altitude $204 \mathrm{~m}$ ) (fig. $10 \mathbf{n}^{\circ} \mathbf{2 2}$ ).

7 Ainsi, en résumé, c'est à l'initiative de Fr. Artaud que des fouilles archéologiques furent pratiquées dans la nécropole tumulaire des environs du château de Verna, notamment sur ce site funéraire campaniforme-Bronze final ${ }^{5}$, mais aussi dans un tumulus voisin fouillé au cours de l'hiver 1818 et dont le mobilier constitua l'essentiel de la collection de Jean-Marie Victor de Verna (1776-1841).

\section{Une tombe à char de la fin du second âge du Fer}

Outre ces quelques pièces de la fin du Néolithique, la collection de Verna est constituée de plus d'une centaine objets ou fragments d'objets, en large majorité en métal (bronze et fer, parfois incrustés d'émail) que l'on désignera, en préalable à toute étude, du nom de « sépulture à char de La Tène finale de Verna ».

\section{La documentation écrite : publication et archives}

\section{L'article du moniteur en 1818}

En fait, immédiatement après sa découverte ${ }^{6}$, la tombe à char de Verna avait été publiée anonymement dans le journal Le Moniteur Universel, daté du 24 décembre $1818^{7}$. L'auteur. de l'article - «un ami des Arts... de Lyon» rapportait la fouille d'un tumulus renfermant les restes "d'un guerrier gaulois qui a vécu à l'époque des colonies romaines", une sépulture tumulaire appartenant à une nécropole plus vaste, à cette date, non explorée et dénommée «Les Quatre Molards» (dans le parler régional «les quatre 
monticules »). Un inventaire sommaire de la trouvaille était alors exposé : casque, deux épées dont l'une pliée, «marmite ", " aiguière » de bronze, fragments de " casseroles ", boucles, fibules, mors en fer et ferrures d'un char (moyeux, clavettes, " accoudoirs »), faux et grenouille en bronze... Cette liste d'objets correspond au contenu de la collection de Verna décrit par les différents visiteurs et aujourd'hui à la Maison du Patrimoine de Hières-sur-Amby, exceptés le casque et quelques objets observés en 1986 par J.-P. Guillaumet.

\section{Les manuscrits : fonds public, collection privée}

La question s'est posée de savoir qui était l'auteur de ce texte.

Deux manuscrits de l'article du Moniteur sont déposés aux archives de l'Académie des Sciences, Belles Lettres et Arts de Lyon (Ms 101/3 et Ms 357) ${ }^{8}$. Ils diffèrent quelque peu de l'article publié puisqu'ils comportent des références à des planches, absentes (chiffres romains, suivi de lettres alphabétiques, parfois redoublées : AA, BB...). Dans le manuscrit 357, intitulé "Extrait du Moniteur", les références ont été rajoutées. Manifestement le texte a été, a posteriori, accompagné des illustrations, précisément de cinq planches figurant les pièces les plus significatives du mobilier funéraire. Le manuscrit a été signé «Artaud », mot qui a été biffé, pour des raisons de place, l'auteur se limitant à écrire l'initiale de son nom de famille.

\section{François Artaud (1767-1838)}

Fr. Artaud, né à Avignon le 17 Avril 1767 dans une famille de drapiers, arrive à Lyon en 1787 afin de se former au métier de dessinateur de soie. Suite à un voyage en Italie, en 1803, où il découvre Pompéi et Herculanum, il renonce à son métier pour se vouer à sa passion : l'archéologie. En 1804, la ville de Lyon acquiert l'abbaye de Saint Pierre, qui devient musée, réunissant les antiquités de la ville. En 1806, Fr. Artaud est nommé inspecteur général du Conservatoire des Arts et antiquaire de la ville (1806-1812), puis sera successivement directeur du Conservatoire des Arts (18121824), puis directeur du musée et de l'Ecole royale des arts de Lyon (1824-1830). Créateur du musée lapidaire, il forme aussi son cabinet particulier, racheté en 1835 par la ville. Le docteur A. Comarmond lui succédera pour la section archéologique du musée. Les ouvrages imprimés, monographies, articles dans la presse locale et régionale, revues scientifiques de Fr. Artaud concernent essentiellement l'archéologie : corpus de mosaïques, fouilles, excursions archéologiques, objets antiques et protohistoriques. De plus, son oeuvre manuscrite est elle aussi importante. Ses carnets, conservés à l'Académie des Sciences et Belles Lettres et Arts de Lyon rassemblent ses observations de fouille. L'ouvrage posthume Lyon souterrain, édité en 1846, est un recueil d'observations archéologiques et géologiques effectuées entre 1794 et 1836 (Bruyère 1986).

C. Bellon 
3- Planche I de la collection privée

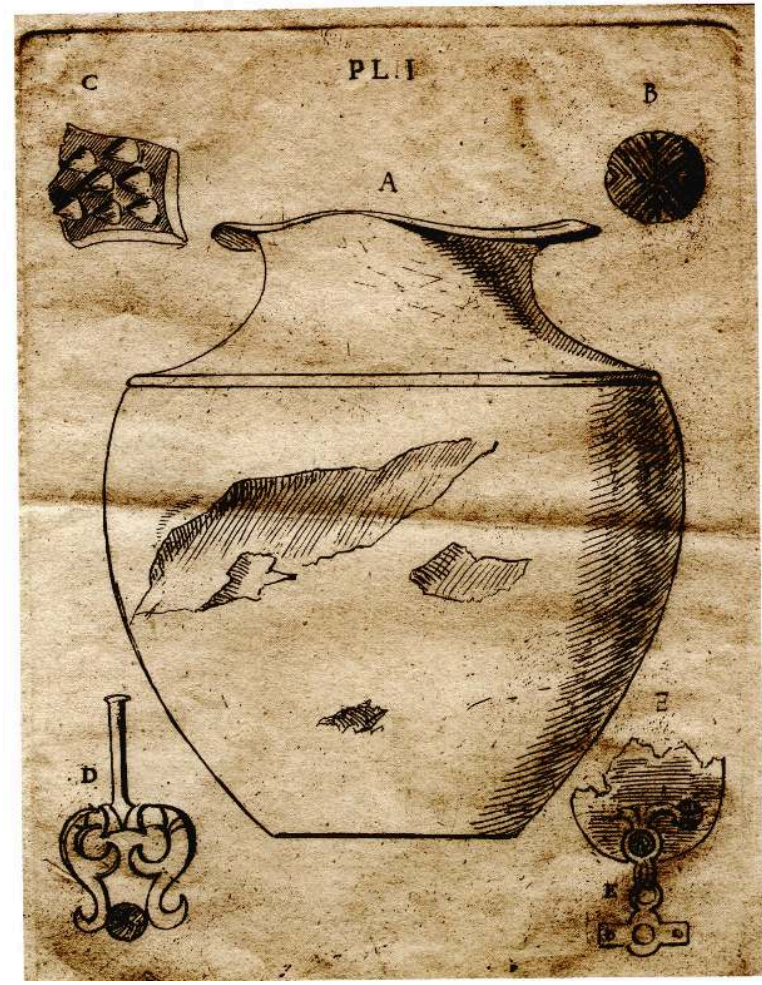

4- Planche III de la collection privée

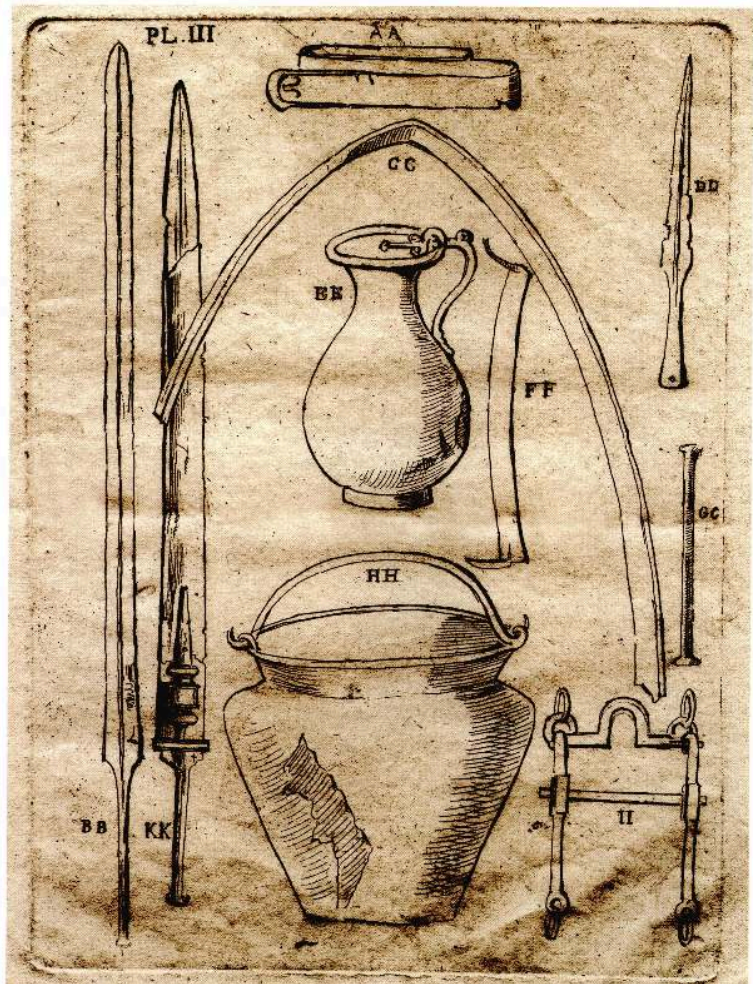




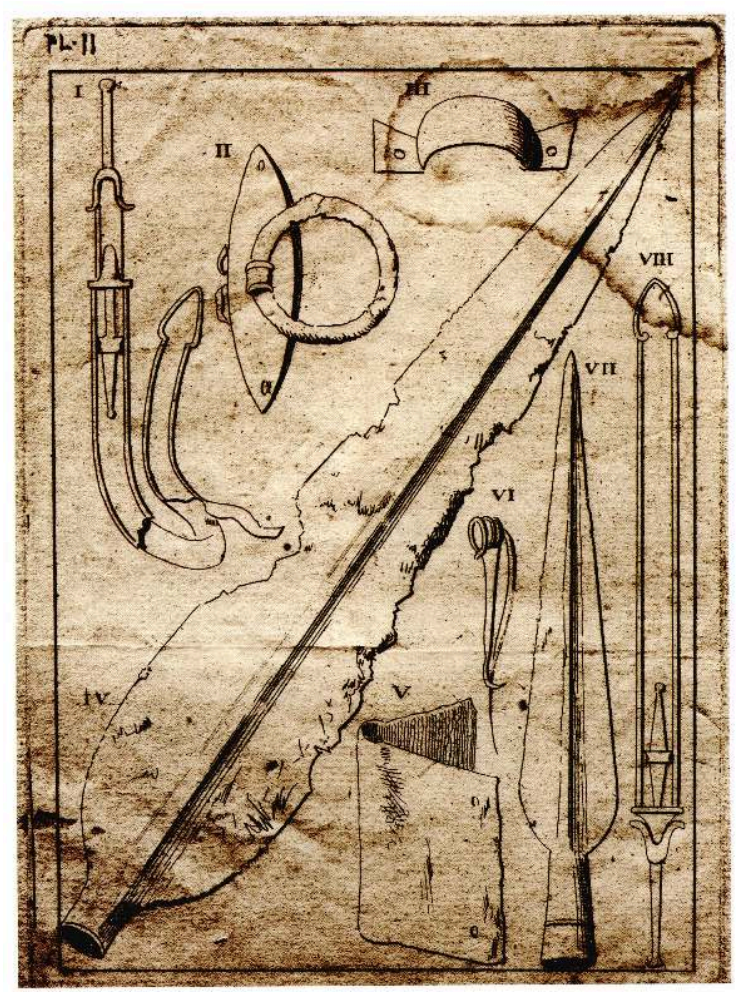

12 Si aucune des planches citées n'est associée au texte, y figure une illustration d'une tombe de cavalier sarmate découverte, sans précision, «sur les bords de la Mer Noire » (preuve supplémentaire de l'intérêt porté par Artaud aux tombes antiques de cavaliers et à chars).

13 Le Ms 101/3, sans titre et sans signature, est légèrement différent dans son introduction et possède quelques mots supplémentaires "ossements animaux" « enseveli avec ses chevaux », des notes, des appels d'illustrations insérés dans le texte. De plus, il contient une liste d'objets numérotés avec leurs dimensions ( $c f$. annexe 1).

Si les planches sont absentes de ces deux manuscrits, par contre deux d'entre elles (III, V) figurent à la fin du manuscrit du Lyon souterrain de Fr. Artaud (Ms 104), associée à une planche supplémentaire particulièrement intéressante (D) ${ }^{9}$. Il s'agit d'une vue générale du tumulus (TVMVLVS en capitales latines) (cf. quatrième de couverture) en fin de fouille sur laquelle celui-ci apparaît à proximité de 3 ou 4 autres tertres de taille variable, d'où l'appellation locale de "Trois ou Quatre Molards» (la variante s'expliquant peut-être par le fait que le plus grand était coupé par une tranchée lui donnant l'apparence de 2 tertres accolés). Manifestement, Fr. Artaud avait fait dessiner le mobilier du tumulus exploré par M. de Verna et gravé des planches dont des tirages sont parvenus entre les mains de l'inventeur de la découverte.

Lors de la publication du Lyon souterrain (1846), l'ouvrage majeur d'Artaud publié après sa mort par l'éditeur Monfalcon - où donc seules deux planches ont été retenues Artaud annonçait la publication prochaine de la tombe à char de Verna :

«Tout cet équipage de mort sera le sujet d'une notice plus détaillée, accompagnée de quelques dessins lithographiés. » (Artaud $1846: 109$ ). 
été ajouté (l'écriture est différente) un bref commentaire : « L'on doit ajouter à cette notice insérée par M. Artaud, Directeur du Musée de Lyon, quelques détails oubliés... » (cf. annexe 1). L'auteur anonyme, probablement le fouilleur, le comte de Verna, atteste ainsi que Fr. Artaud avait été informé immédiatement après la découverte. Sans doute s'était-il rendu sur place puisque dans son Lyon souterrain, Fr. Artaud donne des précisions inconnues ailleurs, en particulier les dimensions du tumulus ( 15 pieds de haut sur 80 de circonférence »).

\section{6- Tirage, non signé, de la lithographie de Lefebvre (Raynard 1819)}

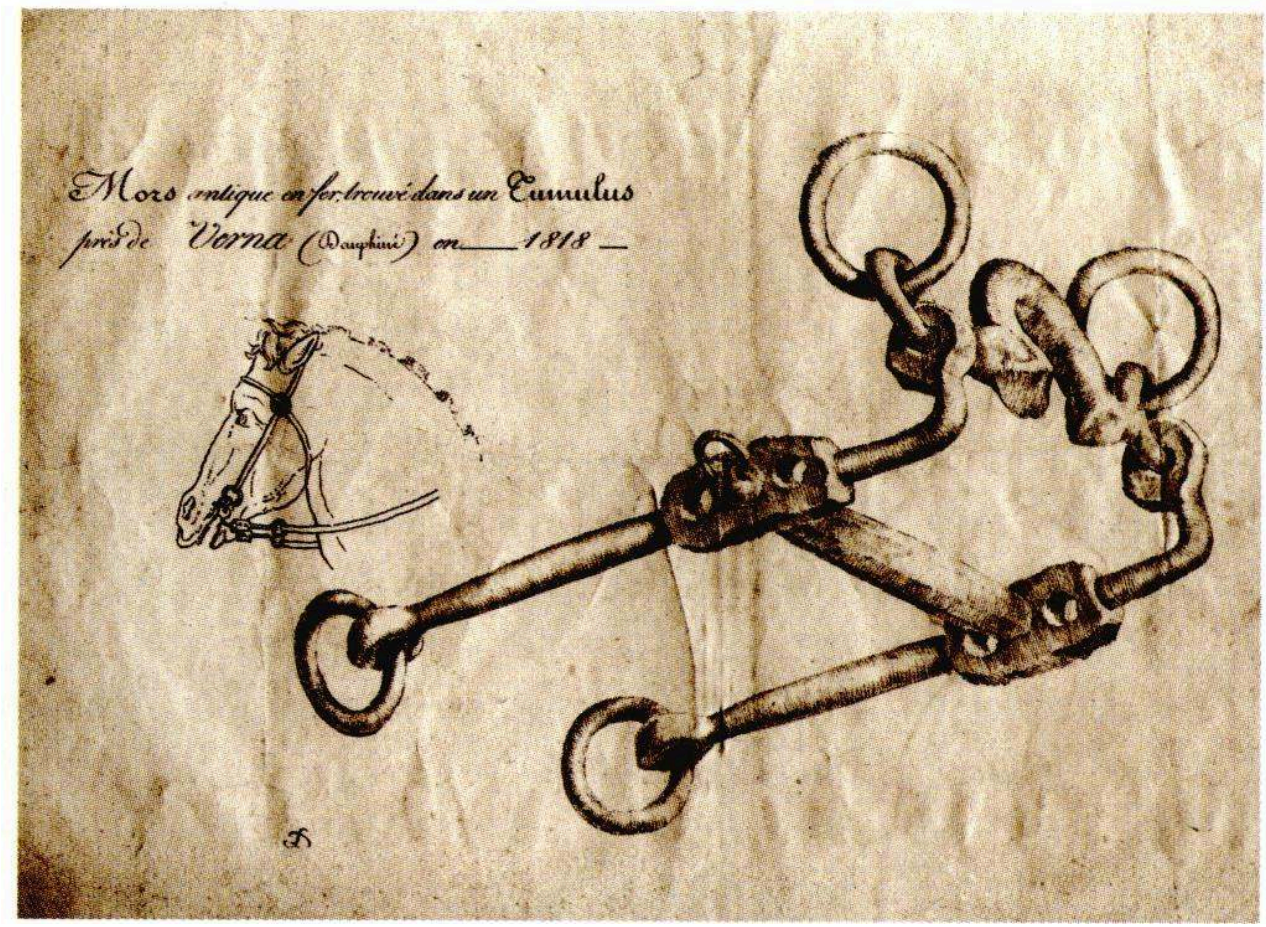

Collection privée

Le dernier manuscrit, certainement une mise au net des autres versions, comporte des appels aux planches insérés dans le texte (idem Ms 101). Certaines planches sont ici présentes (fig. 2 à 5 et $\mathbf{5 2}$ ) pour la première fois, à l'exception de la planche avec le casque. Dans cette collection privée existent une gravure des quatre tumulus et de la sépulture mégalithique (fig. 2), une planche d'objets, notée VIII et réctifiée II (fig. 5) qui n'a pas d'appel dans le texte et des brouillons grands formats figurant des objets du dolmen et des objets de la tombe à char, et enfin le dessin d'un mors.

Une telle découverte ne pouvait être passée inaperçue localement. Effectivement, en 1819, lors d'une séance de l'Académie de Lyon, Fr. Artaud mentionne la trouvaille d'une tombe tumulaire à "Jalionax » en Isère, localisant le site sur l'actuelle commune de Saint-Romain-de-Jalionas, limitrophe de celle de Verna. Seule la présence d'un casque et d'épées était signalée, la communication portant également sur l'existence d'autres tumulus en Dauphiné. La même année, M. Raynard, professeur à l'école vétérinaire de Lyon, publie « recherches sur un mors de bride antique » (Raynard $1819: 193$ ), illustré d'une planche (lithographie de Ch. Lefebvre, Lyon) comportant l'un des mors et une restitution d'un harnachement de Verna (dans laquelle figure un gros cabochon) (fig. 6). 
Quatre-vingts ans plus tard, en 1899, lors de la séance du 1er décembre de l'Académie des Sciences, Belles Lettres et Arts de Lyon, Ernest Chantre (1845-1924), alors sousdirecteur du Museum de Lyon, présente une communication sur «Les nécropoles gauloises du Dauphiné », texte qui reprend celui paru en 1880 dans ses «Etudes paléoethnologiques dans le bassin du Rhône", notice "Leyrieu» (Chantre 1880: 43). L'auteur fouilla dans l'Isle Crémieu dès 1865 (grotte de Bethenas), notamment en 1866 et 1868 , années où ses recherches le conduiront sur le Camp de Larina, près duquel il dit avoir exploré des tumulus protohistoriques (Chantre 1892, 1899, 1900, 1923). Vers 1898, entre deux campagnes en Egypte, il rapporte la découverte de «la nécropole de Leyrieux» - une commune proche de celle de Verna et de Saint-RomaindeJalionas-, « lors de défrichement dans une forêt durant l'hiver 1864... par M. le comte de Verna, propriétaire $d u$ sol». Ignorant manifestement les articles et manuscrits d'Artaud, E. Chantre énumère les objets exhumés selon lui dans un « tombeau... fait de grandes dalles brutes qui ont été prises pour des montants de dolmens »: 4 vases en céramique, 2 en bronze rappelant les oenochoés, 7 grandes épées en fer dont 2 pliées ; parmi celles non pliées une dans son fourreau en fer, mais ornée de bronze, 2 lances en bronze et en fer type La Tène, débris de ceinture et de cuirasses en bronze, nombreux débris de roues en fer, plaques, rondelles, chaînettes et tiges diverses en bronze d'un char.

Il est possible qu'E. Chantre fasse là une confusion avec le mobilier découvert en 1818, bien que les inventaires soient différents. On ne peut exclure l'hypothèse de l'existence d'une deuxième tombe à char qui semblerait toutefois plutôt dater de la transition Bronze-Fer, avec peut-être des tombes adventices de La Tène $C$ et $D$. A l'appui de cette hypothèse, on note que figure dans les collections archéologiques de la Mairie de Crémieu une frette de moyeu du type Wehringen sans provenance, mais sans doute d'origine locale (Bocquet, Haussman 2001). L'objet peut être daté de l'extrême fin de l'âge du Bronze, ou du tout début de l'âge du Fer (bois du char de Wehringen, daté de $778+/-5$ av. J.-C.). On note que de «Crémieu» provient également une épée hallstattienne en bronze (Bocquet 1969: 235). Ainsi, à la fin du XIXe s., l'article du Moniteur est déjà oublié et avec lui la tombe de Verna dont il ne subsiste qu'une collection confuse à l'origine imprécise.

\section{Conclusions}

Que retenir de cette documentation? La fouille eut lieu en novembre 1818 (Artaud 1846 : 107), sur les terres de

"Jalionas, près de Saint-Romain, non loin des bords du Rhône et du château de Verna » (Artaud $1846: 107)$

ou "à peu de distance du Rhône et du château de Verna" (le Moniteur de 1818 et manuscrits). Le tumulus était proche du lieu-dit Les Quatre Molards, appellation locale désignant tumulus, motte et tout relief isolé de ce genre. Sur la planche du manuscrit du Lyon souterrain, légendée "TVMVLVS ", on remarque au premier plan (quatrième de couverture) un tumulus creusé d'une tranchée centrale, et, en arrière-plan, quatre autres tumulus relativement hauts, "proches» du précédent, dont la fouille fut envisagée par M. de Verna. Artaud précise que les sites sont distincts

« les tumuli que l'on voit dans les balmes viennoises, aux Trois-Molards et près du château de Verna, sur un terrain plus élevé que celui qui borde le Rhône » (Artaud $1846: 105)$. 

dernier loue les méthodes de fouille de l'inventeur - «tous les objets trouvés dans le tumulus ont été recueillis avec soin par M. de Verna, amateur éclairé" - remarque particulièrement valable comme le montrera le catalogue des objets conservés. Dans Lyon souterrain, Fr. Artaud donne les dimensions du tertre (1846:107) : 15 pieds de haut pour 80 de circonférence, soit environ $4,5 \mathrm{~m}$ pour un diamètre de 7,60 $\mathrm{m}$ seulement; les gravures montrent effectivement des tumulus hauts et peu érodés, mais leur diamètre semble ne pas correspondre à cette dimension inhabituelle (une confusion entre diamètre et circonférence, soit $24 \mathrm{~m} \times 4,50 \mathrm{~m}$ ?). Selon probablement M. de Verna, la fosse funéraire, carrée, mesurait (cf. annexe 1) « 6 pieds carrés », soit un peu moins de deux mètres de côté et son comblement de charbons et d'ossements " un pied et demi ", soit environ 0,50 cm d'épaisseur. Comme le montre la gravure "TVMVLVS » (D), elle était située dans l'axe médian du tertre, au centre sans doute, bien que ce ne soit précisé nulle part. Selon Fr. Artaud, lors du creusement de la tranchée, au-dessus de la tombe « à fleur de terre » furent découverts des ossements de chevaux, non attribués à la tombe malgré la présence de nombreux éléments de harnachement ensevelis immédiatement « en raison de l'odeur qu'ils dégageaient ».

Quant au mobilier exhumé inventorié, décrit et illustré à la demande de Fr. Artaud, les diverses relations témoignent de sa crémation partielle, parfois violente. Quelques divergences portent sur le nombre d'armes. En effet, le conservateur du Musée des Beaux-Arts mentionne dans tous ses écrits deux épées, l'une repliée l'autre non, alors que M. de Verna parle de «trois sabres » découverts lors de la fouille,

«tous repliés en trois parties... deux de ces fourreaux étaient enfer, et l'autre en cuivre... ». 
Autre variante, les mors de chevaux cités sans décompte dans l'article du Moniteur étaient en fait au nombre de quatre selon M. de Verna (comptage confirmé par Fr. Artaud uniquement dans son manuscrit Ms 101/3). La collection rassemblait un grand nombre d'objets et de fragments d'objets dont la présence étonne à une époque où les techniques de fouilles étaient loin d'être développées. Certains d'entre eux, mentionnés dans les archives ou dessinés lors de la sortie du mobilier en 1986 ne figurent plus dans la collection actuelle du Musée dauphinois : c'est le cas d'un casque en bronze, d'une épée, d'une applique ( ?) en forme de grenouille ${ }^{11}$ (fig. 7), d'un couvercle émaillé, d'une anse anthropomorphe de bassin en bronze et d'une fibule. Si on ne dispose aujourd'hui d'aucune illustration du casque (pl. II disparue), les autres pièces sont connues par des dessins et/ou des photos. Ces pièces semblent conservées chez les héritiers de la collection de Verna.

$\mathrm{Au}$ terme de cette présentation, il semble exclu que la collection de Verna soit un rassemblement d'objets de provenances lointaines. De plus, rien dans les archives ne permet de penser que des objets étrangers aient été introduits dans une découverte locale; toutefois, comme on le verra, la présence d'un bassin étrusque, un vase rare dont la chronologie est distincte de celle de l'ensemble des objets de la sépulture, doit nécessiter quelques explications. Quant à d'éventuels mélanges à partir d'autres découvertes gauloises locales (plusieurs tumuli sont mentionnés et devaient être fouillés), rien ne vient véritablement les confirmer. Naturellement, en l'absence de relevé, il demeure possible - mais cela serait original - que des sépultures adventices de la fin de l'âge du Fer aient fourni du mobilier laténien mélangé ensuite à une éventuelle tombe centrale. Toutefois, la présence, au vu du seul document «de fouille», d'une seule tranchée centrale ne plaide guère en faveur de la fouille d'autres tombes. Non seulement le mobilier intact a été récolté, mais aussi celui réduit à l'état de fragments longs de quelques centimètres à peine, des ossements et de modestes fragments de céramiques. L'explication de cette fouille apparemment méticuleuse figure peut-être dans les textes de Fr. Artaud qui mentionne ce qui semble être l'une des premières comparaisons archéologiques. D'après lui, «le traducteur d'Athénée " certainement Athénée de Naucratis, le célèbre auteur du Banquet des Sages (Deipnosophistes), ajoute que "l'on a trouvé en Amérique cet usage d'élever des tertres ou buttes de terres sur des tombeaux». L'information pourrait provenir de Thomas Jefferson, ambassadeur en France de 1784 à 1789 et futur président des Etats-unis d'Amérique, qui avait exploré en Virginie un des ces mounds des cultures Adena et Hopewell. Sa méthode de travail stratigraphique très en avance sur ce qui se faisait à l'époque a peut-être été diffusée au sein de la communauté des antiquisants de ce temps. Peut-être ses idées sont-elles parvenues jusqu'à Fr. Artaud ou M. de Verna. Quant à l'analyse finale de Fr. Artaud, elle est, comme on le verra, tout aussi remarquable que le fut cette fouille du début du XIXe s. 


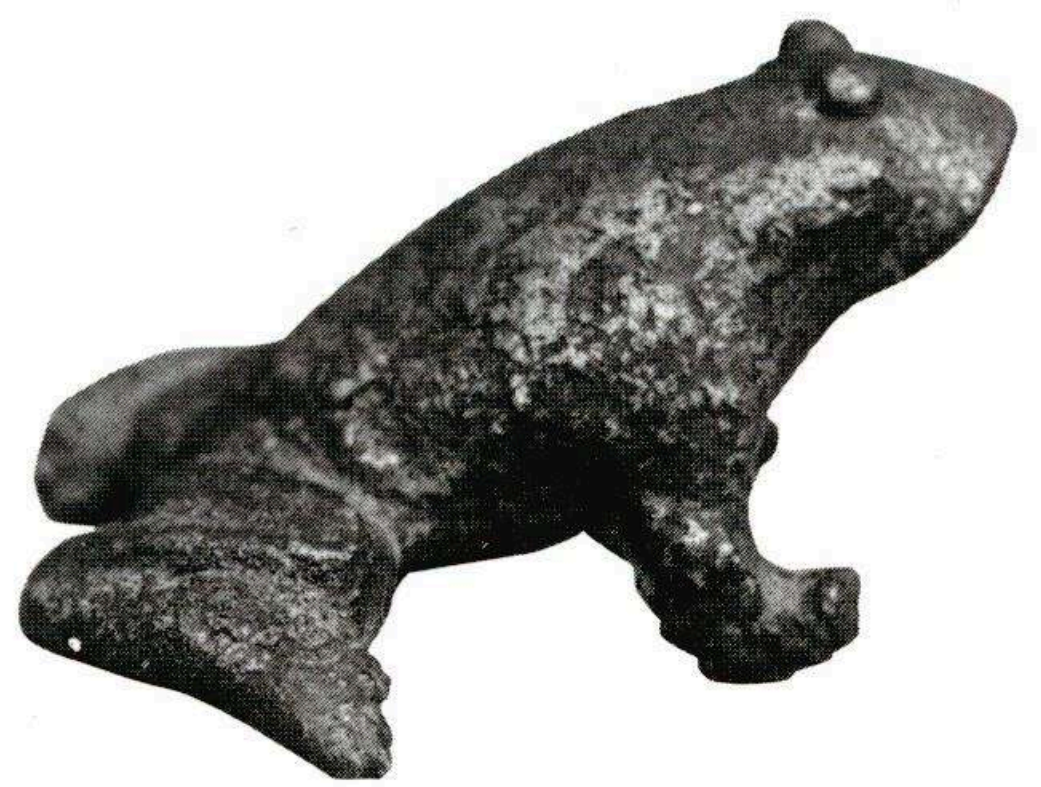

\section{NOTES}

1. Information orale personnelle ; rien, apparemment, n'est conservé dans les notes de Gabriel Chapotat déposées au Centre d'Etudes et de Recherches Archéologiques de Vienne (Isère) ; la documentation de G. Chapotat n'est toutefois pas classée

2. Voir aussi l'annonce de l'étude dans : Verger (S.), Guillaumet (J.-P.) 1988:231.

3. 25 000e IGN, feuille XXXI-31 (Montluel 7-8).

4. BAL, MS 278, P-V. qui contient le résumé de la communication de Fr. Artaud sur les publications de M. Le Penhouet à propos des monnaies armoricaines et des monuments de "Cranac" (sic), lesquels auraient servi de "sépultures de Germains".

5. La parure figure à ce jour dans le mobilier, ainsi que le poignard et le vase campaniforme.

6. On notera que le 20 novembre 1818, à l'initiative du Comte de Montalivet (reprenant un projet de Pierre Legrand d'Aussy), un projet de circulaire-questionnaire sur les monuments et antiquités nationales est discuté par l'Académie des Sciences, Belles Lettres et Arts de Lyon. Faute de crédits, le projet est abandonné, mais en 1819 sera créée la Commission des Antiquités de la France. Ces tentatives d'organiser l'inventaire du patrimoine archéologique explique peut-être la rédaction de la note de Fr. Artaud. Cf. Gran-Aymerich $1988: 36-37$.

7. Le Moniteur Universel, $\mathrm{n}^{\circ} 358,24$ décembre $1818: 1495$.

8. Au dos de l'un d'eux (Ms 357) figure le brouillon d'un texte sur l'emploi du char dans les textes antiques, sans doute dû à Fr. Artaud.

9. Planche du tumulus = Msl04 : fol. 117 vo/p. 224, dite “(pl.D)" ; Planche III = Ms $104:$ fol.117 r $/ p$. 223, dite "pl. II (D bis)" ; Planche V = Ms 104, fol. 106 r\%/p.222, dite “(pl.III)". Ce manuscrit Ms 104 est également déposé à l'Académie des Sciences, Belles Lettres et Arts de Lyon. 
10. Après une longue recherche, les originaux dont des copies sont conservées à la Maison du Patrimoine de Hières-sur-Amby ont été retrouvés chez l'actuel héritier de la famille de Verna qui avait mis en vente les objets. Ce dernier nous a aimablement transmis les documents qu'il avait en sa possession, à savoir textes et dessins, mais a également confirmé détenir quelques objets conservés comme souvenirs.

11. Si la grenouille n'a pas figuré dans la vente, elle est toutefois connue par un jeu de photographies réalisées par J.-P. Guillaumet (fig. 7). 


\title{
Chapitre 2. Le contexte de la découverte
}

\author{
Franck Perrin et Robert Royet
}

8- La costière ouest de l'Isle Crémieu

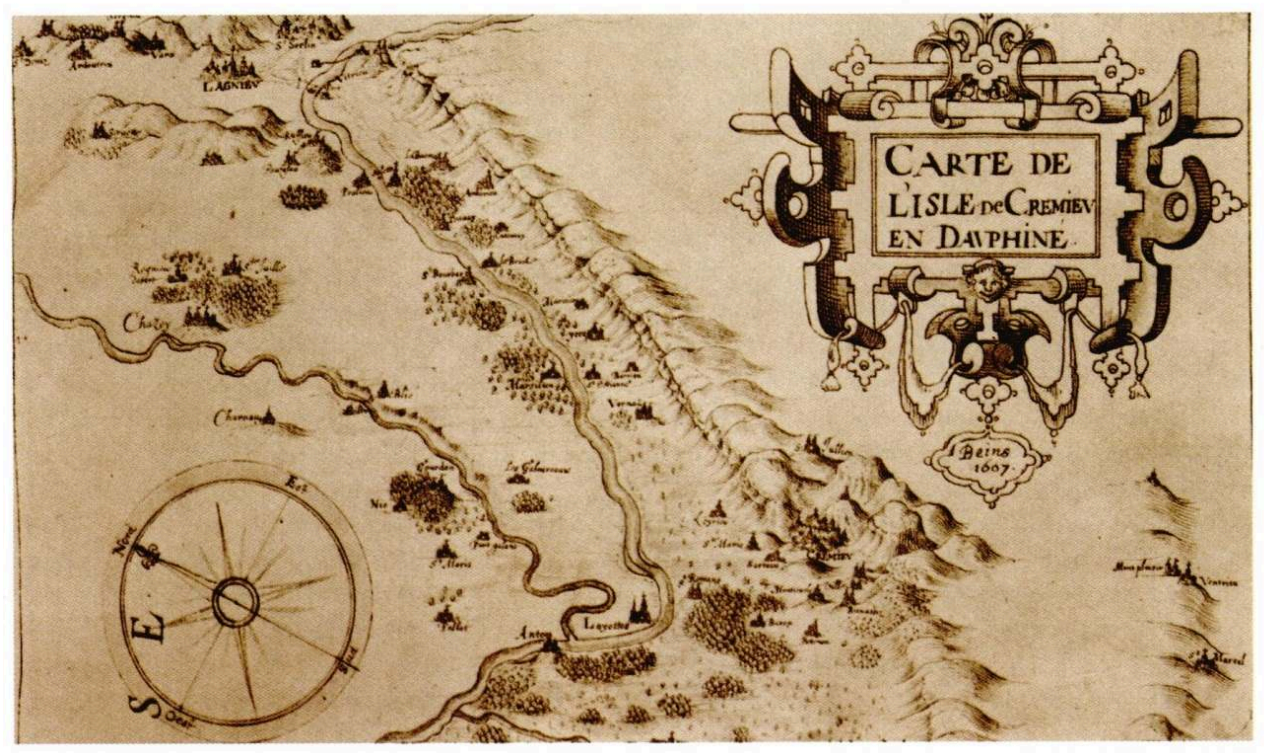

D’après Jean de Beins, 1607

\section{Les allobroges à la fin de l'âge du Fer}

Mentionnés dès le IIIe s. av. J.-C. à propos du passage de l'expédition d'Hannibal, les Allobroges, et notamment les principaux personnages de ce peuple, sont ensuite régulièrement cités par les auteurs gréco-latins à propos d'événements guerriers des IIe-Ier s. av. J.-C. Les informations disponibles tant sur l'époque à laquelle fut enseveli le 
guerrier de Verna que sur la classe sociale à laquelle il appartenait sont toutefois bien minces.

Les Allobroges comme bien d'autres Gaulois semblent avoir connu des systèmes politiques oligarchiques, antérieurement à la conquête. Hannibal, selon Polybe, se heurta à des " chefs de secteurs " allobroges et non à un roi ou roitelet. Strabon, utilisant les Histoires de Poséidonios, indique que la noblesse allobroge transforma Vienna, métropolis (ville mère) des Allobroges de simple village en ville (polis). Il est probable que cette évolution décisive a été réalisée avant la conquête de la Transalpine. En 121 av. J.-C., l'attaque par les Allobroges et le refus de ces derniers de livrer le roi salyen Teutomalus conduisent à la perte de leur indépendance. Seul Appien mentionne un roi allobroge nommé Bituitos; les fastes triomphaux conservent quant à eux le souvenir des défaites « des Allobroges » et du roi (Betultus) des Arvernes. A priori il s'agit d'une erreur d'Appien confondant Arvernes et Allobroges, mais on ne peut totalement écarter l'hypothèse d'une homonymie plus ou moins complète amenant une certaine confusion dans les sources littéraires; il est également possible que Bituitos, Arverne, ait été roi des Allobroges, ce type de royauté étant plusieurs fois attesté, tant en Gaule qu'en Bretagne. Alors que chez leurs alliés arvernes, la royauté disparaît, aucun changement notable ne semble concerner les Allobroges. L'aristocratie de ce peuple nous est connue grâce à Cicéron qui, dans sa défense du gouverneur Fontéius, critique Indutiomaros, ambassadeur des Allobroges et des autres provinciaux. Malheureusement, à part quelques attaques sur la barbarie gauloise, son discours n'apporte guère d'éléments à la connaissance des élites allobroges de cette époque.

Même remarque à propos des députés allobroges envoyés à Rome en 63 av. J.-C. Seul Plutarque mentionne l'envoi de lettres aux Allobroges, ce qui laisse penser que certains d'entre eux lisaient le latin (Indutiomaros affirmant qu' « il savait » comment s'était enrichi Fontéius, dut s'exprimer en latin). L'année suivante, un certain Catugnatos sera " chef de toute la nation » pour le dernier soulèvement allobroge. Cette magistrature fut sans doute strictement militaire, le personnage étant éventuellement désigné par le peuple réuni en assemblée. Selon César, Abducillos, père de deux fils qui combattirent à son côté pendant la guerre des Gaules, fut « à la tête de sa nation durant de longues années ». Pourtant les Allobroges disposaient à cette époque d'un sénat; César y fera admettre à titre exceptionnel Ecos et Roucillos, les deux fils d'Abducillos. Le fonctionnement de ce sénat était-il analogue à celui des Eduens? Chez ce peuple, l'entrée au sénat était réservée à un membre de chaque famille, le père excluant ses fils; César aurait donc usé de son autorité sur une institution provinciale pour contourner cet usage. Toutefois, chez les Eduens, le magistrat supérieur Vergobretos ne pouvait occuper qu'une seule année sa charge, la cédant à un autre selon " un antique usage ». Mais en ce temps-là (le début du Ier s. av. J.-C.), Abducillos représentait sans doute un parti romain; César récompensera ses fils en leur accordant «les plus hautes magistratures ", sans malheureusement les citer (Goudineau 2002 : note 5). 


\section{Choix de textes antiques sur les allobroges (fin Ile- milieu ler s. av. J.-C.)}

Les campagnes romaines de 121 av. J.-C. Tite-Live, Abrégés, Livre LXI (123-120 av. J.-C.)

Éd. P. Jal, CUF

4 Le proconsul C. Sextius, après avoir vaincu la peuplade des Salyens, fonda la colonie d'Aquae Sextiae, ainsi appelée en raison à la fois de l'abondance des eaux provenant des sources chaudes et froides et de son propre nom à lui. Le proconsul Cn. Domitius remporta un succès sur les Allobroges, près de la ville de Vindalium. Si on leur avait fait la guerre, c'est parce qu'ils avaient recueilli Toutomotulus, roi des Salyens, en fuite, et lui avaient apporté toute l'aide qu'ils pouvaient; parce qu'ils avaient aussi ravagé le territoire des Eduens, alliés du peuple romain. (...)

5 Le consul Q. Fabius Maximus, petit-fils de Paulus, lutta victorieusement contre les Allobroges et Bituit, le roi des Arvernes. De l'armée de Bituit, 120000 hommes furent tués; comme le roi lui-même s'était rendu à Rome pour donner réparation au Sénat, on l'envoya à Albe pour être détenu, parce qu'il semblait contraire à la paix de le renvoyer en Gaule. On décida aussi d'arrêter son fds Congonnetiacus et de l'envoyer à Rome. On reçut la soumission des Allobroges.

6 Le consul Q. Fabius Maximus gagna le surnom d'Allobrogique grâce à sa victoire.

7 Autres récits de ces épisodes avec anecdotes: Strabon, Géographie, IV, 1, 11; Velleius Paterculus, Histoire romaine, II, 10 et 39 ; Valère Maxime, Faits et dits mémorables, III, 5, 2 ; VI, 9, 4 ; IX, 6, 3 ; Pline l'Ancien, Hist. Nat., VII, 166 ; Suétone, Néron, II, 1 ; Florus, Epitomé, I, 37 ; Appien, Celtica, I, 2 ; Eutrope, Abrégé, IV, 10 ; Orose, Contre les Païens, V, 13, 2 ; V, 14, $1-4$.

Vienne (fin lle s. av. J.-C. ?)

Strabon, Géographie, IV I, II (Cl86),

Éd. F. Laserre, CUF

8 Les Allobriges, autrefois, participaient à des opérations guerrières par dizaines de milliers, mais aujourd'hui ils cultivent les plaines et les vallons des Alpes. Ils vivent dans des villages, mais les plus illustres d'entre eux vivent à Vienne. De ce qui était auparavant un village portant pourtant le titre de métropole de ce peuple, ils ont fait une ville.

\section{Bituitos, roi des Allobroges? (121 av. J.-C.)}

\section{Appien, Histoire romaine IV. Celtique, Frgt 12 2-5.}

\section{Éd. P. Viereck, A. G. Roos, E. Gabba, Teubner}

$\mathrm{Au}$ moment où il (Gnaius Domitius) traverse le territoire des Salyens, vient à sa rencontre un ambassadeur du roi des Allobroges Bituitos, en riche équipage; des gardes du corps l'accompagnaient en tenue d'apparat et des chiens; car les Barbares de cette région ont une escorte constituée de chiens. Un musicien chantait, sur une 
musique barbare, le roi Bituitos, puis les Allobroges, puis l'ambassadeur lui-même, célébrant sa naissance, son courage et sa richesse. C'est pour cette raison surtout que ceux des ambassadeurs qui sont illustres emmènent ces gens.

\section{La conjuration de Catilina (63 av. J.-C.) \\ Salluste, La conjuration de Catilina, 40-41 \\ Éd. A. Ernout, CUF}

10

Il (Lentulus) charge donc un certain P. Umbrenus de s'aboucher avec les députés des Allobroges, et de les déterminer, si possible, à s'allier à lui, dans la pensée que ces hommes accablés sous le poids de leurs dettes publiques et privées, et de nature belliqueuse comme le sont tous les Gaulois, se rallieraient aisément à un tel dessein. Umbrenus, qui avait fait du négoce en Gaule, était connu de la plupart des chefs de cités et les connaissait lui-même. Aussi sans perdre de temps, dès qu'il aperçut au forum les députés Allobroges, après quelques brèves questions sur l'état de leur cité, feignant de s'apitoyer sur leur sort, il leur demanda quelle issue ils espéraient à de tels maux. Quand il les voit se plaindre de la cupidité des magistrats, accuser le Sénat de leur refuser toute assistance et n'attendre d'autre remède que la mort à leurs misères : « Eh bien moi, dit-il, si seulement vous voulez être des hommes, je vous montrerai le moyen d'échapper à tous ces maux. » A ces paroles, les Allobroges, pleins d'espoir, supplient Umbrenus d'avoir pitié d'eux; il n'était rien de si pénible, de si ardu qu'ils ne fussent prêts à faire avec enthousiasme, pour délivrer leur cité de la dette qui l'accablait. Umbrenus les amène dans la maison de D. Brutus, car elle était toute proche du forum, et n'ignorait rien du complot grâce à Sempronia: Brutus, lui, était alors absent de Rome. Il fait en outre venir Gabinius, pour donner plus de poids à ses propos. En sa présence, il expose la conspiration, nomme les conjurés, auxquels il ajoute les noms de nombreuses personnes de toute espèce, absolument innocentes, afin d'affermir le courage des députés gaulois ; leur concours une fois promis, il les renvoie chez eux.

11 Cependant les Allobroges hésitèrent longtemps sur le parti à prendre. Ils mettaient en balance d'un côté leurs dettes, leur amour de la guerre, les grands avantages que laissait espérer la victoire ; de l'autre la supériorité des forces, l'absence de risques, et au lieu d'une espérance douteuse des récompenses assurées. Après de mûres réflexions, ce fut la Bonne Fortune de la République qui finit par l'emporter. Aussi révèlent-ils toute l'affaire, comme ils l'avaient apprise, à Q. Fabius Sanga, le protecteur attitré de leur cité. Cicéron, instruit par Sanga du projet qui se trame, enjoint aux députés de feindre un zèle ardent pour la conjuration, de se mettre en rapport avec tous les autres complices, de leur faire de belles promesses et de tâcher de les amener à se découvrir le plus possible.

\section{La révolte de Catugnat (62/61 av. J.-C.) Dion Cassius, Histoire Romaine, XXXVII47-48, Éd. E. Gros, Firmin-Didot}

Les Allobroges commettaient des dégâts dans la Gaule Narbonnaise. C. Pomptinus, gouverneur de cette province, envoya contre eux ses lieutenants : quant à lui, il campa dans un lieu d'où il pouvait observer tout ce qui se passait; afin de leur donner, en toute occasion, des conseils utiles et de les secourir à propos. Marius Lentinus se mit en 
marche contre Ventia et il effraya tellement les habitants que la plupart prirent la fuite : le reste lui envoya une députation pour demander la paix. Sur ces entrefaites, les gens de la campagne coururent à la défense de la ville et tombèrent à l'improviste sur les Romains. Lentinus fut forcé de s'en éloigner; mais il put piller la campagne sans crainte, jusqu'au moment où elle fut secourue par Catugnat, chef de toute la nation, et par quelques Gaulois des bords de l'Isère. Lentinus n'osa dans ce moment les empêcher de franchir le fleuve; parce qu'ils avaient un grand nombre de barques : il craignit qu'ils ne se réunissent, s'ils voyaient les Romains s'avancer en ordre de bataille. Il se plaça donc en embuscade dans les bois qui s'élevaient sur les bords du fleuve, attaqua et tailla en pièces les barbares, à mesure qu'ils le traversaient; mais s'étant mis à la poursuite de quelques fuyards, il tomba entre les mains de Catugnat lui-même, et il aurait péri avec son armée, si un violent orage, qui éclata tout à coup, n'eût arrêté les barbares.

Catugnat s'étant ensuite retiré au loin en toute hâte, Lentinus fit une nouvelle incursion dans cette contrée et prit de force la ville auprès de laquelle il avait reçu un échec. L. Marius et Servius Galba passèrent le Rhône, dévastèrent les terres des Allobroges et arrivèrent enfin près de Solonium. Ils s'emparèrent d'un fort situé audessus de cette place, battirent dans un combat les barbares qui résistaient encore et brûlèrent quelques quartiers de la ville dont une partie était construite en bois: l'arrivée de Catugnat les empêcha de s'en rendre maîtres. A cette nouvelle, Pomptinus marcha avec toute son armée contre Catugnat, cerna les barbares et les fit prisonniers, à l'exception de Catugnat. Dès lors, il fut facile à Pomptinus d'achever la conquête de ce pays...

\section{Les nobles allobroges Roucillos et Ecos (58-48 av. J.-C.) César, Guerre civile, III 59,1-2,}

\section{Éd. L.-A. Constans, CUF}

Il y avait, auprès de César, dans la cavalerie, deux frères allobroges, Roucillus et Aécus, fils d'Adbucillus, qui avait été pendant de longues années à la tête de sa nation; c'étaient des hommes d'un courage extraordinaire, de qui César avait éprouvé dans toutes les campagnes des Gaules les services éminents et la très grande vaillance. Pour cette raison, il leur avait confié dans leur patrie les plus hautes magistratures, il s'était occupé de les faire nommer à titre exceptionnel au sénat, il leur avait distribué des terres gauloises prises à l'ennemi ainsi que de grosses récompenses en argent, et de pauvres qu'ils étaient, ils les avaient rendus riches.

\section{L'Isle Crémieu : géographie et données archéologiques}

\section{Le contexte géographique}

15 L'Isle Crémieu se situe à l'extrémité nord du département de l'Isère, à une quarantaine de kilomètres à l'est de l'agglomération lyonnaise et à une cinquantaine au nord-est de Vienne (fig. 8 et 9). Ce massif calcaire jurassique est entouré au nord, à l'est et à l'ouest par le cours du Haut-Rhône et délimité au sud par les cours de la Bourbre et de la Save ainsi que par de grandes zones de marais, d'où son surnom d'Isle. 
9- Proposition de localisation du dépôt funéraire de Verna

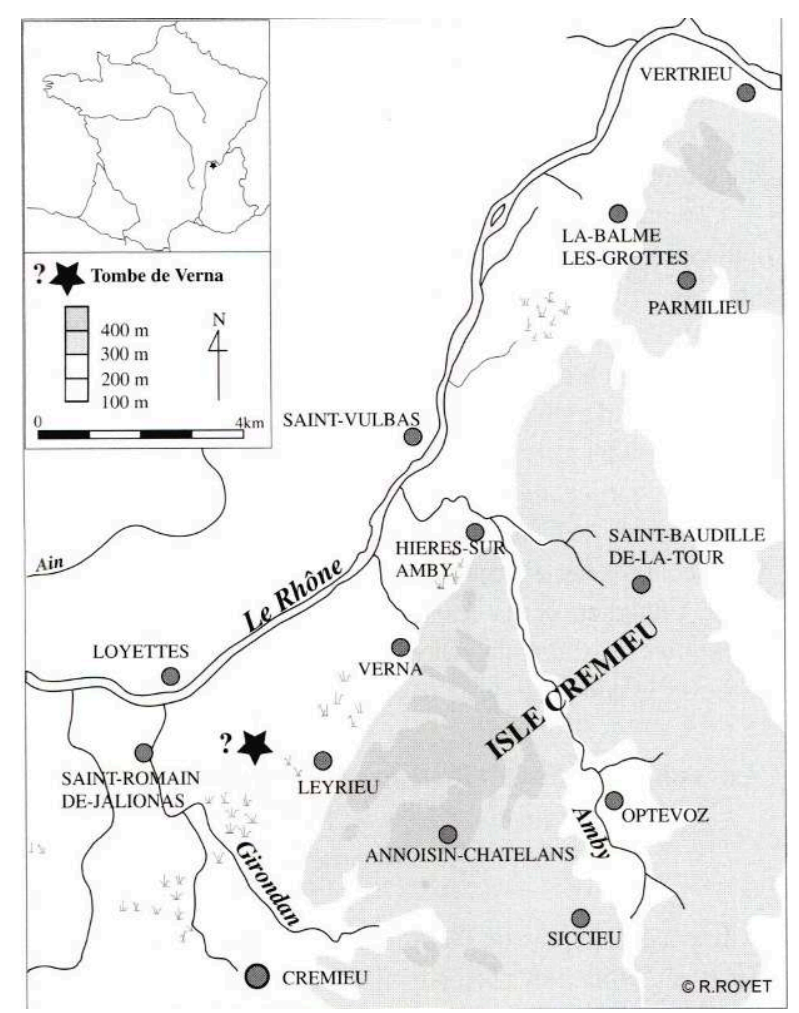

Plateau triangulaire de $225 \mathrm{~km}^{2}$, il est entaillé par des vallées qui en permettent l'accès à l'ouest, notamment le val d'Amby, qui, après avoir longé le plateau de Larina, un éperon délimité sur deux côtés par des falaises, débouche au coeur du massif. Les falaises dominent de 200 mètres le cours du Rhône, distant d'un kilomètre ; c'est dans cette plaine, non loin de l'occupation protohistorique du Camp de Larina qu'a été mis au jour la tombe à char de Verna (Perrin 1990 : 12-13).

Historiquement et géographiquement, l'Isle Crémieu dépend de la cité allobroge. Elle s'inscrit dans une situation périphérique et frontalière à la cité des Ambarres. Avec la défaite des Allobroges en 121 av. J.-C. et la création de la Provincia en 118 av. J.-C., leur territoire est intégré à cette dernière. Par ailleurs, le plateau de l'Isle Crémieu occupe une position particulière à la frontière entre la Gaule Chevelue et la province de Narbonnaise. La rive droite du Rhône, aujourd'hui dans le département de l'Ain, pose des problèmes d'attribution ethnique, puisqu'une partie de cette zone pourrait être rattachée au territoire allobroge, notamment Saint-Vulbas. Le monnayage gaulois recueilli sur le plateau de Larina et plus largement dans l'Isle Crémieu (cf. annexe 2) confirme clairement le lien de dépendance de ce secteur géographique vis à vis du pouvoir allobroge.

\section{Le plateau de Larina}

Dominant les plaines du Rhône et de l'Ain, le plateau de Larina (Hières-sur-Amby, Isère) est assurément le site archéologique majeur de ce secteur de l'Isle Crémieu; avec 27 hectares enclos dans une fortification de près d'un kilomètre, le camp de Larina est aussi le plus vaste site du territoire allobroge, juste après Vienne la capitale, un habitat sans fortification reconnue, estimé à une quarantaine d'hectares. A Larina, l'espace circonscrit par le rempart s'organise autour d'une vaste doline cultivable jouxtant un 
plateau et entourée de collines plus propices à l'élevage. Les premières recherches datent du XIXe s. (E. Chantre, F. Gabut...). Après une longue interruption, elles ont repris dans le courant des années 1950, puis à l'occasion de destructions occasionnées par des carrières d'extraction de lauzes. Les travaux les plus importants ont porté sur un habitat, une nécropole et une chapelle de l'Antiquité tardive et du haut Moyen Age établis à l'extrémité nord du site. Fouilles, sauvetages urgents et prospections de surface ont révélé l'ancienneté de la fréquentation du site. En effet, outre quelques témoins du Néolithique, une quinzaine de structures fossoyées ovalaires ou circulaires et des lambeaux de niveaux de sol en place, ont été observées par J.-R Pelatan et P. Porte. Le mobilier recueilli (plus de 6000 fragments de vases uniquement dans les fouilles gallo-romaines et médiévales) permet d'envisager une occupation du plateau aux débuts du Bronze Final (Fosse M), mais surtout aux phases IIIa et IIIb du Bronze Final (Xe-IXe s. av. J.-C.) (Froquet 1994; Pélatan 1986). L'existence de fours " polynésiens » et de fosses-dépotoirs sans structures de stockage ni vestiges d'habitats pourraient laisser penser à un espace à vocation spécialisée, éventuellement un lieu de rassemblements festifs en marge d'un habitat détruit par les carrières, ou lié à des habitats dispersés dans les alentours du site de Larina. De plus, dans une faille - celle de La Chuire - située dans la falaise défendant naturellement le site, à l'aplomb de la tourbière de Verna et en contrebas de la doline centrale, un important mobilier du Bronze Final (III essentiellement) a été exhumé à l'occasion d'une série de sondages portant sur environ $60 \mathrm{~m}^{2}$ (Perrin 1990).

Sur le plateau, le Premier âge du Fer est individualisé par de rares structures datées par la méthode $\mathrm{du}{ }^{14} \mathrm{C}$. La faille de La Chuire a également livré du mobilier du début du Ville s. av. J.-C. et de la fin du VIe s. av. J.-C., notamment pour cette époque des importations provençales et massaliotes. La fréquentation du site semble s'arrêter vers la fin du Ve s. av. J.-C., car aucun mobilier ne peut assurément être rapporté aux phases Hallstatt D3 et La Tène A et B. Dans la faille de La Chuire, un important mobilier des phases B2 à D1 de La Tène constitué de près de 400 objets en métal et de plusieurs milliers de fragments de céramiques provient des sondages pratiqués dans les années 1970/1980. Ce mobilier métallique est surtout constitué d'objets de la vie quotidienne (parures, outils, ustensile de cuisine) intacts, souvent peu usagés et parfaitement réutilisables ; quelques pièces (fourchette, faux, fibules, gouge, gril, fourreau probablement...) présentent des destructions assurément volontaires, analogues aux manipulations reconnues sur des objets funéraires et cultuels. La question se pose de savoir s'il s'agit du produit d'un sanctuaire non localisé situé sur le plateau ou d'un dépôt dans un site naturel remarquable dépourvu - du moins en apparence - d'aménagements construits (Perrin 1990).

20 Parallèlement, aucun document du IIIe s. av. J.-C. n'a été à ce jour identifié sur le plateau. Sur ce dernier, plusieurs structures ainsi que du mobilier récolté hors contexte lors des fouilles et des prospections réalisées dans la doline centrale permettent de cerner une occupation laténienne durant la phase D1. Environ un millier de fragments d'amphores républicaines, de la vaisselle campanienne à vernis noir, des vases en bronze, plusieurs dizaines de monnaies allobroges et massaliotes ainsi qu'une trentaine de bracelets en verre caractériseraient cet horizon. 


\section{Le contexte archéologique}

21 Selon toute vraisemblance, le Rhône délimite la frontière du territoire allobroge au nord du Dauphiné (Rémy 2000), les éventuelles possessions de ce peuple mentionnées par César (B.G., I. 11) sur la rive droite ne paraissant être que des têtes de pont.

Malgré cette situation de frontière, cette portion de la vallée du Rhône est un important noeud de communication et de passage. Cette vallée, qui se rétrécit très rapidement du sud au nord, forme un couloir de circulation naturelle qu'il convenait de contrôler. En effet le Rhône en amont de Lyon est facilement navigable pour les bateaux à faible tirant d'eau, au moins jusqu'au verrou de Sault-Brenaz, situé à une quinzaine de kilomètres en amont de Saint-Romain et où se situe le point de rupture de charge. L'Antiquité gallo-romaine a ainsi légué plusieurs témoignages de nautes et d'utriculaires assurant notamment l'approvisionnement de Lyon (épitaphe de M. R. CATULLUS à Murs et Gélignieux, C.I.L., XIII, 2494). La vallée est aussi sillonnée par plusieurs itinéraires routiers plus ou moins bien identifiés.

23 Entre La Balme-les-Grottes et Saint-Romain-de-Jalionas, au moins trois points de passage du fleuve sont utilisés pendant l'Antiquité sans que l'on connaisse exactement leur importance réelle (Royet, Faucher 2002). Au nord, sur la commune de La Balme-lesGrottes (hors figure), deux gués ou aménagements permettaient traditionnellement le passage du Rhône. Le plus septentrional traverse l'Ile de Saint-Vulbas pour aboutir à La Balme-les-Grottes au lieu-dit Sous le Pré du Seigneur ; le second relie le coeur du gisement antique de Saint-Vulbas Les Sétives, au lieu-dit Sous le Château, vers le hameau de La Brosse. Sur la commune de Hières-sur-Amby, il est possible qu'un passage ait existé entre le Port du Noyer et le hameau de Marcilleux (lieu-dit Le Prieuré) sur la rive droite (fig. $10 \mathbf{n}^{\circ}$ 16). Une récente monographie consacrée au site de Saint-Vulbas (Vaireaux 1996) rappelle toutefois l'absence de certitudes archéologiques sur l'ancienneté de ces points de passage. Par contre, l'utilisation durant la Protohistoire du gué de Port SaintOyand, entre Saint-Romain-de-Jalionas et Loyettes, ne fait l'objet d'aucune incertitude (fig. 9 et $10 \mathbf{n}^{\circ}$ 3). En effet, si les aménagements de berge détectés en prospection (rapport, Convert 1994) ainsi que plusieurs routes qui y convergent en provenance de Vienne et de Lyon ne datent peut être que de l'époque gallo-romaine, ce gué sert de centre de gravité à la nécropole tumulaire à laquelle appartient la tombe de Verna (cf. infra). Il permettait aux voies provenant du Jura ou de la Saône de traverser aisément le Rhône et ainsi de rejoindre la route de l'Italie passant au sud du plateau. 
10- Tombes protohistoriques fouillées et habitats laténiens sur les communes de Saint-Romain-deJalionas, Leyrieu, Verna, Hières-sur-Amby

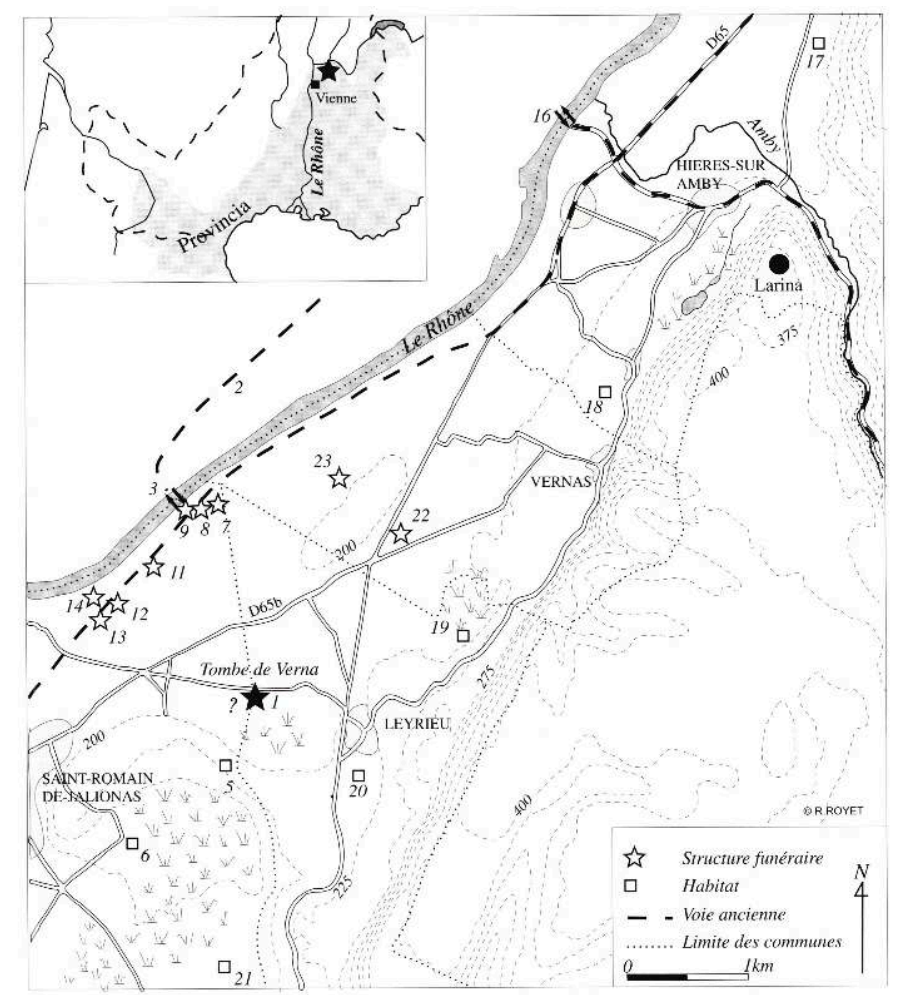

1 : tombe de Verna, 2 : voie ancienne, $3:$ gué de St Oyand, $5:$ habitat du Perrier, $6:$ Le Vernai, $7:$ tumulus Géraud, 8 : tumulus Nicole, 9 : tumulus Roger, 11 : Enclos des Tâches, 12 : tumulus du Peillard, 13 : gravière BétonRhône, 14 : tumulus Stéphane, 16 : gué du port de Noyer, 17 : habitat de Marignieu, 18 : habitat de Mollard Reynard, 19 : habitat de Pré Moly, 20 : habitat des Plantées, 21 : habitat de SainteMarie-de-Tortas, 22 : tombe du Général Romain, 23 : La Tour Berthet, (pour les sites n, 10 et 15 cf. fig. 11)

Les axes de circulation terrestres protohistoriques et antiques restent en partie hypothétiques. L'axe S-O/N-E le plus connu, longe le fleuve en reprenant peu ou prou le chemin dit du Peillard à Saint-Romain-de-Jalionas puis l'assiette de la route départementale $65 \mathrm{~h}$, le long de laquelle sont installés les hameaux de Travers et de La Brosse (fig. $10 \mathbf{n}^{\circ}$ 2). Il est partiellement visible sur la photo aérienne au niveau du lieudit Les Tâches, à Saint-Romain-de-Jalionas. Cette route suit à peu près le talus de séparation des basse et moyenne terrasses. Subsistant aujourd'hui sous forme de chemins vicinaux discontinus, cet itinéraire présentait jadis un intérêt régional. Sur le cadastre napoléonien il est, par exemple, désigné comme voie menant de Lyon à Lagnieu. Faute de fouille, sa datation reste problématique. Son tracé s'est légèrement déplacé au cours des temps. Aux Tâches, où il est localisable grâce à la trace laissée par ses deux fossés bordiers, il recoupe un enclos circulaire. Quelques centaines de mètres plus à l'ouest, il semble au contraire que le fossé laténien du tumulus Stéphane se soit installé contre le chemin (fig. $10 \mathbf{n}^{\circ} \mathbf{1 4}$ ).

Aucun indice archéologique ou morphologique ne permet de restituer un second itinéraire parallèle longeant les falaises de l'Isle Crémieu. Les hypothèses de voies pénétrantes qui se dirigeraient vers le plateau sont encore moins assurées. Il est vraisemblable que des axes empruntaient les combes d'Amblérieu et surtout d'Amby qui permettent une montée facile vers le sommet du plateau où était installé l'important oppidum de Larina (fig. 10), mais les routes actuelles paraissent modernes. 
Le chemin qui, actuellement, mène presque en ligne droite du bord du Rhône au val d'Amblérieu parait totalement indifférent aux sites protohistoriques ou gallo-romains. Plus au sud, à Hières-sur-Amby, la route qui relie le Port du Noyer, où existait un bac à l'époque moderne, au Val d'Amby suit les courbes de niveaux. Là encore, elle n'est associée à aucun contexte archéologique précis.

Contrairement à la voie longeant le fleuve, ces itinéraires ne possèdent qu'une fonction de desserte locale et ne pouvaient vraisemblablement pas constituer un raccourci pour des voies commerciales inter-régionales. Le gain de distance et de temps procuré par un raccourci passant par le plateau et se dirigeant vers un gué en amont par rapport à la voie bordant le fleuve paraît dérisoire ; il serait largement annulé par les difficultés engendrées par le relief d'une part et par la traversée des dépressions marécageuses occupant le centre du plateau d'autre part.

Le réseau des implantations humaines est dominé par l'oppidum de Larina qui occupe l'éperon au débouché du Val d'Amby (cf. supra).

28 Curieusement, l'habitat perché et fortifié dans l'Isle Crémieu, que le Rhône longe sur près de $50 \mathrm{~km}$, paraît secondaire. Une prospection systématique du rebord occidental du plateau n'a pas permis de déceler un seul autre établissement fortifié de hauteur alors que plusieurs sites paraissaient, a priori, très favorables. Sur le rebord oriental du plateau, qui n'a toutefois pas fait l'objet d'un examen détaillé, aucune découverte protohistorique n'attire l'attention sur un site éventuel.

Grâce aux prospections réalisées ces dernières années, il est par contre possible d'identifier au moins sept habitats laténiens de plaine. Faute de fouilles extensives, il convient de rester prudent dans l'estimation de leur importance et dans leur interprétation.

Sur les communes de Saint-Romain, Leyrieu et Hières-sur-Amby, quatre sites galloromains importants de la fin du Ier s. av. J.-C., Le Vernai, Marignieu, Pré Moly et Sainte Marie-de-Tortas (fig. $10 \mathbf{n}^{\circ} 6,17,19,21$ ) ont été précédés par un établissement de tradition indigène. Parmi eux, seul le site du Vernai à Saint-Romain, à $2 \mathrm{~km}$ au sud-ouest de l'emplacement présumé de la tombe dite de Verna, a fait l'objet de fouilles sur une surface assez importante (rapport, Royet et al. 2001 ; Royet 2002). Des niveaux et des vestiges de construction en matériaux légers y ont été étudiés sur $1500 \mathrm{~m}^{2}$, mais des sondages suggèrent une extension sur au moins un hectare. Les fouilles montrent que la rupture architecturale entre un établissement laténien et une villa gallo-romaine précoce est contrebalancée par une permanence dans les domaines alimentaires, économiques et culturels. Ce constat suggère un maintien et une romanisation de l'aristocratie allobroge plutôt que l'arrivée d'une nouvelle population de colons. Le matériel assez abondant permet de proposer pour cet établissement indigène une occupation durable entre la 2e moitié du IIe s. et le milieu du Ier s. av. J.-C. Cet établissement rural, le seul fouillé dans le secteur étudié, ne résulte donc pas d'une réorganisation agricole consécutive à la conquête. Les sites de Mollard Reynard (fig. 10 $\mathbf{n}^{\circ}$ 18), des Plantées (fig. $10 \mathbf{n}^{\circ}$ 20) et du Perrier (fig. $10 \mathbf{n}^{\circ}$ 5) n'ont en revanche pas survécu à la romanisation.

31 L'établissement du Vernai semble être représentatif d'une catégorie d'habitats de plaine durables où résiderait l'aristocratie allobroge locale. Ces établissements constituent les principaux centres de peuplement. Le scénario du passage d'un habitat de hauteur laténien à un réseau de villae gallo-romaines en plaine ne semble pas pertinent dans cette microrégion. Il n'a pas été repéré de gisement évoquant une proto-agglomération. 
Seul le supposé vicus d'Optevoz, au centre du plateau, a fourni des indices localisés d'occupation préromaine sans que l'on sache s'il s'agit de traces d'un habitat groupé ou isolé.

On peut s'interroger sur les modalités d'exploitation agricole de cette plaine. Les travaux récents sur le marais de Grand Plan à Saint-Romain-de-Jalionas (rapport, Royet et al. 2001) ont mis en évidence les témoins d'une mise en culture de ces terres limoneuses aujourd'hui marécageuses dès le IIe s. av. J.-C. En fait, l'analyse sédimentaire montre qu'une pluviométrie alors moins importante a permis le développement de cultures céréalières (blé amidonnier et surtout orge) dans des dépressions qui ont connu une paludification à partir du Ier s. av. J.-C.

Ces habitats situés à l'intérieur des terres, souvent au bord d'une dépression cultivable, ont en commun d'éviter les sols fersiallitiques trop perméables. Sur ce type de sols, de nombreux monuments funéraires ont été identifiés et quelquefois fouillés.

\section{La nécropole tumulaire}

Les découvertes de monticules funéraires protohistoriques se sont multipliées sur les communes de Saint-Romain-de-Jalionas, Leyrieu et Verna depuis près de deux siècles. En bordure du Rhône, ces tombeaux se concentrent dans un espace de deux kilomètres d'ouest en est, entre le Peillard à Saint-Romain et la Tour Berthet à Verna, à l'écart des habitats contemporains (fig. $10 \mathbf{n}^{\circ} \mathbf{1 2}$ et 23). L'ancienneté de certaines découvertes liée à l'absence d'une approche globale de cet espace ont généré une certaine confusion. Il n'est donc pas inutile d'en rappeler la succession.

\section{Historique des découvertes}

Outre les découvertes du XIXe s. (cf. supra), et en particulier celle de la tombe de Verna en 1818, puis celle du Général Romain (fig. $10 \mathbf{n}^{\circ}$ 22), après un arrêt des recherches de plus d'un siècle, plusieurs tumulus ont fait l'objet de recherches ces trente dernières années.

En 1971, J. Rebillard repère, lors d'une prospection aérienne, un monticule de $8 \mathrm{~m}$ de diamètre entouré d'un fossé (fig. $10 \mathbf{n}^{\circ}$ 23) au lieu-dit La Tour Berthet à Verna (rapport, Rebillard 1971). La fouille, annoncée, ne sera jamais effectuée.

Au cours du printemps 1971, des labours font apparaître des dalles au nord-ouest de la tombe du Général Romain. Un sondage de contrôle mené par J. Rebillard ne permet pas de distinguer de vestiges en place.

La même année un sauvetage est organisé sous la direction de A. Bocquet pour réétudier la tombe du Général Romain (Bocquet 1976) elle-même. Cette allée couverte d'origine chalcolithique connaît deux phases d'agrandissement ultérieures, peut-être datables du Bronze ancien et du Bronze final.

En 1974, Ch. Revellin (rapport. Revellin 1974) fouille le tumulus dit du Peillard. Il découvre alors une incinération associée à quelques fragments métalliques non datés (fig. $10 \mathrm{n}^{\circ}$ 12).

40 A. Dufourg ouvre durant l'été 1976 une tranchée de reconnaissance à travers le tumulus dit des Trois Tilleuls ou tumulus Géraud (fig. $10 \mathbf{n}^{\circ}$ 7), à Saint-Romain, qu'il attribue à la fin de l'âge du Bronze (Dufourg, Antoine 1978) grâce à trois tessons trouvés dans le 
remplissage. Il détecta à cette occasion les traces d'une fouille antérieure, mais n'atteignit pas la chambre sépulcrale.

41 Un décapage dans la gravière BétonRhône, à quelques mètres à l'ouest du tumulus du Peillard, entraîne l'organisation d'un sauvetage dirigé par P. Porte (inédit) durant le printemps 1986 (fig. $10 \mathbf{n}^{\circ}$ 13). Un ou plusieurs enclos quadrangulaires laténiens auraient été fouillés à cette occasion.

En 1987, J.-P. Guillaumet et S. Verger, après une tentative infructueuse sur l'oppidum de Larina, reprennent la fouille du tumulus des Trois Tilleuls (rapport, Verger, Guillaumet 1987). Il fut rebaptisé tumulus Géraud ou Tombe du Prince celte après la découverte d'un ensemble remarquable daté du Bronze final IIIb comportant des armes et de la vaisselle de bronze (Verger, Guillaumet 1988) (fig. $10 \mathbf{n}^{\circ}$ 7).

Le même été, ils examinent un tertre voisin distant d'une centaine de mètres (fig. $10 \mathbf{~ n}^{\circ}$ 9), baptisé Roger (rapport, Verger, Guillaumet 1987).

Une troisième butte, dénommée Abel, située à la pointe sud du Bois des Tâches est identifiée, mais n'est pas fouillée (fig. $11 \mathbf{n}^{\circ} \mathbf{1 0}$ ).

Ce programme d'exploration est poursuivi en 1990 par S. Hettiger qui sonde alors quatre structures (rapport, Hettiger 1990):

- Le tumulus $d u$ Peillard, déjà fouillé par Ch. Revellin, qui se révèle entouré d'un fossé dont la base du comblement comporte des tuiles; il semble que cet enclos ait été installé sur une structure fossoyée circulaire plus ancienne visible sur une photo aérienne.

- Le tumulus Stéphane (fig. $10 \mathbf{n}^{\circ}$ 14), à $100 \mathrm{~m}$ au nord-ouest du précédent.

- Le tumulus Nicole (fig. $10 \mathbf{n}^{\circ}$ 8), à proximité du tumulus Géraud.

- Enfin l'examen d'un cercle repéré au lieu dit Les Tâches, visible sur les photos aériennes (fig. $\left.10 \mathbf{n}^{\circ} 11\right)$, n'est pas couronné de succès.

A. Bocquet et L. Haussmann ont étudié en 1998 plusieurs pièces retrouvées par J.-R Jospin dans les réserves de la mairie de Crémieu. Cet ensemble comportait, entre autres, un fragment de frette de moyeu et deux pièces de décoration pouvant provenir d'un char de la fin de l'âge du Bronze final ou du début du Hallstatt. Un fragment de bracelet à godrons hallstattien et une statuette en fer « italique » attribuée au VIe-Ve s. complètent ce lot. Les circonstances de leur découverte et leur provenance est inconnue. Une découverte locale est toutefois probable. Aucun tumulus n'ayant été retrouvé sur la commune de Crémieu, il est vraisemblable que ces pièces proviennent d'une cachette de fondeur ou, plus probablement, d'un ou plusieurs tumulus situés aux alentours de la tombe de Verna (Bocquet, Haussmann 2001).

En 1999, des campagnes d'évaluation sont réalisées à l'occasion de l'agrandissement de la gravière Verdolini (ex BétonRhône), $100 \mathrm{~m}$ au sud-ouest des précédentes découvertes. Les sondages sont tous négatifs (rapport, Hénon 1999).

\section{Organisation de la nécropole}

Seuls neuf tumulus ou enclos, éparpillés sur $2 \mathrm{~km}^{2}$, ont fait l'objet de recherches sur lesquelles on dispose d'archives qui permettent aujourd'hui de les localiser. L'importance de certaines des découvertes (tombe de Verna et tumulus Géraud) ne doit pas masquer la méconnaissance du contexte funéraire dans lequel elles s'inscrivent. Il faut recourir à la photo-interprétation pour suppléer partiellement aux lacunes de la 
documentation. Malheureusement, cette zone est spécialisée dans la céréaliculture intensive. Les missions récentes de l'I.G.N. ne fournissent donc que peu d'informations.

Une partie seulement de la nécropole est couverte par une mission ancienne à grande échelle ${ }^{12}$, qui permet néanmoins de déceler dix-huit enclos indétectables par prospection pédestre. Un croquis d'interprétation de ces photos (fig. 11) montre que la nécropole tumulaire s'étend sur la basse et la moyenne terrasses et occupe notamment les terrains fersiallitiques délaissés par l'habitat. A l'ouest, elle est cantonnée par des bras d'un ancien cours d'eau se jetant dans le Rhône. Il semble que ce paléochenal appartienne à la Bourbre, qui actuellement coule $5 \mathrm{~km}$ plus à l'ouest, plutôt qu'au Girondan, petit affluent du Rhône qui utilise aujourd'hui la même confluence (Bernigaud 2002) que ces bras anciens. Le recensement des sites en bord de ce cours paraît indiquer qu'il fonctionnait pendant l'âge du Bronze. Le déplacement du lit et l'atterrissement de ces bras interviendraient antérieurement au IIe s. av. J.-C. A l'est, la limite de la nécropole est plus floue et ne correspond à aucun obstacle topographique. La tombe dite du Général Romain (fig. $10 \mathbf{n}^{\circ}$ 22) est à ce jour la découverte la plus orientale et, avec la tombe de La Tour Berthet, la seule située sur la commune de Verna. Cette tombe du Général Romain se distingue, par son ancienneté, des autres sépultures du secteur. On ne peut donc exclure qu'elle se situe hors de l'emprise du reste de la nécropole. Toutefois, au sud-ouest de cette tombe, plusieurs micro-reliefs peuvent, éventuellement, signaler la présence de sépultures arasées.

Entre ces points extrêmes, les tombes paraissent, à première vue, essentiellement localisées près du Rhône, à la limite des terrasses basse et moyenne. En fait, cette répartition le long d'un axe de circulation ancien est artificiellement accentuée par des conditions de visibilité différentes. En premier lieu, il faut rappeler que la limite entre les deux terrasses est encore partiellement boisée et moins intensément cultivée que la partie centrale de la plaine. Les témoins archéologiques y sont donc aujourd'hui plus facilement repérables. En second lieu la lisibilité des vestiges sur les photographies n'est pas partout similaire: presque aucun tumulus n'est visible à l'est du bois des Tâches alors que plusieurs y ont été fouillés. Ce secteur se révèle aussi très pauvre en traces de parcellaires antiques par ailleurs très bien conservés à l'ouest du bois (fig. 11 $\mathrm{n}^{\circ} \mathbf{4 )}$. Les conséquences de cette différence de visibilité sont d'autant plus importantes que cet ensemble a été l'objet de fouilles ponctuelles, mais jamais d'une tentative de reconnaissance systématique.

51 Au nord, la voie ancienne ne borde pas la nécropole. Contrairement à l'assertion reprise par certains fouilleurs, la terrasse basse du Rhône livre plusieurs tombes, notamment vers l'ouest.

Enfin, l'extension vers le sud est problématique. Comme à l'est du bois, le laniérage du parcellaire au sud de la route départementale D65b, de création récente, contribue à masquer les vestiges. Un enclos rectangulaire est néanmoins visible en bordure de la route départementale $65 \mathrm{~h}$ dans une parcelle plus large que la moyenne (fig. $11 \mathbf{n}^{\circ} 15$ ). Autour de cet enclos, plusieurs cercles sont éparpillés dans l'espace compris entre les D65b et $65 \mathrm{~h}$.

53 Par contre aucune tombe n'a été décelée sur les reliefs ou, au contraire, dans les dépressions humides en bordure desquelles se trouve l'habitat laténien. Vernes (fig. 10, 11), correspond à l'extrémité des sols fersiallitiques, au plus près des 
terrains cultivés et habités. A l'intérieur du périmètre circonscrit l'organisation de l'espace est très mal connue. La seule contrainte topographique est le talus entre les terrasses du Rhône. Le problème du tracé exact de la voie protohistorique a déjà été évoqué.

\section{1- Photo-interprétation du secteur des Tâches à Saint-Romain-de-Jalionas}

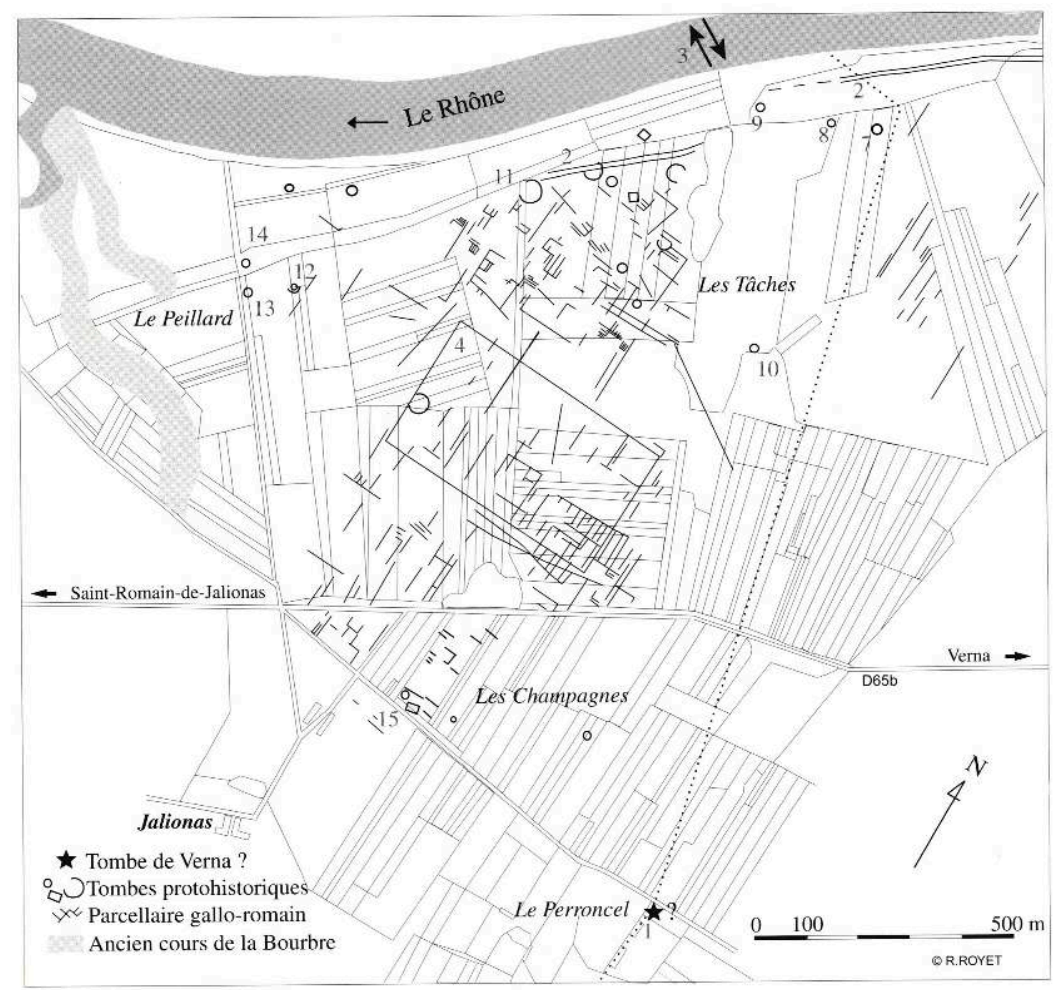

1 : localisation proposée pour la tombe de Verna, 2 : Voie ancienne, $3:$ Gué de St Oyand, 4 : Parcellaire gallo-romain, 7 : tumulus Géraud, 8 : tumulus Nicole, 9 : tumulus Roger, 10 : tumulus $A b e l, 11$ : enclos des Tâches, 12 : tumulus du Peillard, 13 : gravière BétonRhône, 14 : tumulus Stéphane, 15 : Les Champagnes, (pour les sites $n^{\circ} 5$ et 6 cf. fig. 10)

\section{Synthèse des données issues des fouilles}

Les recherches ont été trop partielles et trop aléatoires pour que l'on puisse dresser une typologie ou restituer des regroupements familiaux. En fait, chaque tumulus est unique par sa structure comme par son dépôt (tab. 1).

\section{Phasage}

Les tombes identifiées lors de fouilles peuvent être regroupées en trois phases.

La plus ancienne, représentée uniquement par la tombe du Général Romain, correspond au Chalcolithique (fig. $10 \mathbf{n}^{\circ} \mathbf{2 2}$ ).

La seconde phase, à la fin de l'âge du Bronze, comprend le tumulus Géraud et les tumulus Nicole et Roger ( $\mathbf{n}^{\circ} 7,8$ et 9$)$, tous trois concentrés immédiatement à l'est du gué des Taches. Un second groupe peut être restitué un kilomètre à l'ouest. A proximité de l'ancien cours de la Bourbre, les tumulus dits du Peillard et Stéphane ( $\mathbf{n}^{\circ} 12$ et 14) semblent comporter un état ancien. Leur datation précise est toutefois aléatoire. 
59 La tombe de Verna ( $\left.\mathbf{n}^{\circ} \mathbf{1}\right)$ appartient à la troisième période, datable de La Tène. Un deuxième ensemble, à $200 \mathrm{~m}$ à l'ouest, est repéré en bordure de la route menant à Leyrieu, au lieu-dit Les Champagnes (fig. $11 \mathbf{n}^{\circ}$ 15). Associé à trois cercles, on distingue un enclos fossoyé rectangulaire de $22 \mathrm{~m} \mathrm{x} \mathrm{16,5} \mathrm{m.} \mathrm{Il} \mathrm{peut} \mathrm{être} \mathrm{rapproché} \mathrm{de} \mathrm{l'enclos}$ funéraire collectif laténien de la Noue Mauroy à Acy-Romance (Lambot et al. 1994).

Un troisième groupe d'enclos quadrangulaires comprenant les tumulus Stéphane et du Peillard ( $n^{\circ} 12$ et 14 ), ainsi que les enclos fouillés dans la gravière BétonRhône, se dessine à l'ouest. Au cours de cette dernière phase il semble que plusieurs tumulus anciens fassent l'objet d'une restauration et d'un réemploi. La nécropole paraît connaître alors son extension maximale.

61 Faute de fouilles, l'homogénéité et la datation du groupe de neuf enclos repérés immédiatement à l'ouest du bois des Tâches reste hypothétique. Il est vraisemblable que la proximité du gué a attiré très tôt l'installation de tombes, mais la forme quadrangulaire d'un des enclos pourrait se rapprocher des tombes laténiennes connues par ailleurs. Le recoupement de certains de ces enclos par les fossés de la voie d'origine ancienne ne peut guère être retenu comme indice de datation, cette route ayant pu subir des modifications de tracé limitées et tardives.

62 Il convient en tout cas de ne pas dresser trop vite un parallèle entre les emprises et les significations des deux phases d'enfouissement. S'il est possible d'associer la nécropole de l'âge du Bronze à l'oppidum de Larina, selon un schéma rencontré au premier âge du Fer dans d'autres gisements (Vix - Mont Lassois), il ne semble pas pertinent d'en faire de même pour la nécropole de l'âge du Fer. L'organisation sociale est alors très différente et le peuplement n'est pas concentré dans l'oppidum, mais éclaté dans la plaine.

\section{La tombe de Verna : proposition de localisation}

63 L'emplacement du tumulus a été décrit de façon très approximative dans les différents comptes rendus relatant la fouille (Le Moniteur du 24 décembre 1818) (cf. supra). Il n'existe évidemment aucun report cadastral et les hypothèses de localisation émises depuis lors ne s'accordent même pas sur la commune de découverte.

Les travaux de synthèse les plus récents (rapports, Verger, Guillaumet 1987 ; Hettiger 1990) suggèrent, sans plus de précision, de le situer aux abords du château de Verna, propriété du fouilleur. Cette proposition repose, d'une part, sur une confusion entre le nom de l'inventeur et la commune où est implantée son domaine et, d'autre part, sur la proximité supposée de la tombe du Général Romain, fouillée au même moment par le même M. de Verna. Cette allée couverte campaniforme a été réexaminée et précisément localisée (Bocquet 1976) sur la commune de Verna au lieu-dit Le Charnier à $750 \mathrm{~m}$ à l'ouest du château.

65 En fait, une approche régressive des documents anciens associée à une vérification de terrain permet de proposer une nouvelle localisation, aux confins des communes de SaintRomain-de-Jalionas et de Leyrieu (fig. 10). Le compte-rendu d'Artaud situait explicitement la découverte sur les terres de Jalionas, une maison forte du XIVe s., et non chez M. de Verna. Le domaine de Jalionas occupait, au XIXe s., la partie nord-est de l'actuelle commune de Saint-Romain-de-Jalionas et la pointe septentrionale de Leyrieu, immédiatement au nord-est. Il ne s'est, par contre, jamais étendu jusqu'aux alentours du château de Verna qui était entouré de vastes possessions. 

par la planche de 1818 (quatrième de couverture). Sur ce document qui représente la fouille au premier plan, le château de Verna, au second plan, se découpe devant les falaises du plateau de l'Isle Crémieu. Cette représentation, avec le corps de bâtiment construit au XVIIIe s. devant le donjon médiéval, correspond à une vue depuis le sudouest. Cet angle d'observation exclut à la fois les piémonts de l'Isle Crémieu et le secteur des Tâches et du Peillard au nord de Saint-Romain-de-Jalionas, où l'on rencontre la plus grande densité de tumulus ( $c f$. infra). Dans ce dernier cas, le château de Verna serait, de plus, invisible parce que caché par le bois des Tâches déjà présent sur le cadastre napoléonien. Pour les mêmes raisons, il n'est pas non plus possible de retenir une localisation en bordure du Rhône, à l'est du bois des Tâches, dans le secteur de l'actuel toponyme Les trois Mollards, au voisinage duquel Artaud situe la tombe (dans l'esprit de ce dernier, ce toponyme pouvait s'appliquer à toute cette portion, monotone, de la plaine entre Saint-Romain et Saint-Etienne-d'Hières).

Ce mamelon, qui a attiré l'attention des archéologues dès le début du XIXe s., devait être suffisamment visible pour avoir servi de point de repère dans ce paysage plat et déboisé.

Tableau 1 - Synthèse des données issues des fouilles

\begin{tabular}{|c|c|c|c|c|c|c|c|}
\hline Numéro & Nom/année de fouille & Tertre & Fossé & Rite & Mobilier & Datation & Bibliographie \\
\hline 22 & $\begin{array}{l}\text { Tombe du Géneral Romain } \\
1818 ; 1971\end{array}$ & $\begin{array}{l}\text { Allée couverte } \\
\text { avec } 2 \text { phases } \\
\text { d'agrandissement de } \\
\text { la chambre }\end{array}$ & & Inhumation & $\begin{array}{l}\text { Poignard a languette et } \\
\text { gobelet campaniforme, } \\
\text { pointe de flèche, } \\
\text { plat à cannelures, } \\
\text { tuiles, céramique }\end{array}$ & $\begin{array}{l}\text { Chalcolithique/Bronze Ancien } \\
\text { Bronze Final II b ou III a } \\
\text { Bas-Empire? }\end{array}$ & Bocquet 1976 \\
\hline 14 & $\begin{array}{l}\text { Tumulus Stéphane } \\
1990\end{array}$ & $\begin{array}{l}\text { Remblai circulaire } \\
\text { de } 12 \mathrm{~m} \text { de dia. } \\
\text { et d'1 } \mathrm{m} \text { de haut }\end{array}$ & $\begin{array}{l}\text { Trapézooidal de } 24 \mathrm{~m} \text { de côté } \\
\text { et } 1.80 \mathrm{~m} \text { de profondeur, } \\
2 \text { etats de comblement }\end{array}$ & ? & Céramique & $\begin{array}{l}\text { Néolithique } \\
\text { ou âge du Bronze }\end{array}$ & Inédit \\
\hline 12 & $\begin{array}{l}\text { Tumulus du Peillard } \\
\qquad 1974 ; 1990\end{array}$ & $\begin{array}{l}\text { Butte irrégulière de } \\
14 \mathrm{~m} \times 11 \mathrm{~m}, \\
\text { haute de } 1,50 \mathrm{~m}\end{array}$ & 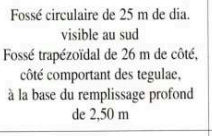 & Crémation & $\begin{array}{l}\text { Anneaux et clous } \\
\text { en fer et } \\
\text { tôles de bronze, } \\
\text { charbons }\end{array}$ & $\begin{array}{l}\text { Âge du Bronze? } \\
\text { Fin de lâge du Fer? }\end{array}$ & Inédit \\
\hline 9 & $\begin{array}{l}\text { Tumulus Roger } \\
\quad 1987\end{array}$ & $\begin{array}{l}\text { Butte de sable de } 25 \mathrm{~m} \\
\text { de dea. } \\
\text { haute de } 0,80 \mathrm{~m} \text {. } \\
\text { Couronne dorthostates. } \\
2 \text { pierres sur le sommet } \\
\text { du tertre }\end{array}$ & $\begin{array}{l}\text { Fossé circulaire "peu profond" } \\
\text { non décrit }\end{array}$ & Crémation & $\begin{array}{l}\text { Céramiques (urne et couvercle), } \\
\text { anneaux en alliage à } \\
\text { base cuivre à forte teneur en étain }\end{array}$ & Âge du Bronze (III b) & $\begin{array}{l}\text { Mention in Verger, } \\
\text { Guillaumet 1988: } \\
\text { 239. Mobilier } \\
\text { inédit }\end{array}$ \\
\hline 8 & $\begin{array}{l}\text { Tumulus Nicole } \\
1990\end{array}$ & $\begin{array}{l}\text { Butte circulaire de } \\
16 \mathrm{~m} \text { de dia. } \\
\text { haute de } 1,60 \mathrm{~m}\end{array}$ & $\begin{array}{c}\text { Fossé circulaire de } 23 \mathrm{~m} \text { de dia. } \\
0,80 \mathrm{~m} \text { de profond. } \\
\text { Couronne d'orthostates } \\
\text { en périphérie }\end{array}$ & $\begin{array}{l}\text { Inhumation } \\
\text { sous dalles }\end{array}$ & $\begin{array}{l}\text { Céramiques, } \\
\text { épingle en bronze }\end{array}$ & Âge du Bronze (III b) & Inédit \\
\hline 7 & $\begin{array}{c}\text { Tumulus Géraud } \\
\text { ou "du prince celte" } \\
\text { ou "Des Trois Tilleuls" } \\
\text { 1976, } 1987\end{array}$ & & & Inhumation & $\begin{array}{l}\text { Orfèverie, } \\
\text { vaisselle métallique, } \\
\text { panoplie guerrièrc, } \\
\text { faune }\end{array}$ & Âge du Bronze (III b) & $\begin{array}{l}\text { Verger. } \\
\text { Guillaumet 1988; } \\
\text { étude en cours }\end{array}$ \\
\hline 11 & Enclos des Tâches & $\begin{array}{l}\text { Fossé circulaire } \\
\text { de } 35 \mathrm{~m} \text { de dia.. }\end{array}$ & & & & ? & \\
\hline
\end{tabular}

Une description du secteur est fournie par une enquête détaillée de délimitation des territoires respectifs des mandements de Saint-Romain-de-Jalionas et de Crémieu datant de 1460. Les limites utilisent en général soit des chemins soit des points remarquables du paysage où sont alors placées des bornes. Un point se singularise toutefois au lieu-dit Le Perroncel où fut implantée une borne (fig. 11), imposant à cette frontière un tracé qui n'est pas rectiligne et ignore tout du parcellaire et des chemins qu'il coupe en oblique. On peut supposer que les officiers delphinaux chargés du découpage ont utilisé un point remarquable préexistant dans le paysage, vraisemblablement un tumulus. Aujourd'hui, la parcelle est recouverte d'un bosquet, ce qui rend malaisé le contrôle du terrain, mais le sol y est plus élevé que dans les parcelles avoisinantes, et il présente de surcroît un aspect chaotique pouvant résulter 
de terrassements anciens. Cette proposition de localisation, issue d'un faisceau de présomptions concordantes, reste donc hypothétique ${ }^{13}$.

\section{NOTES}

12. Mission CDP 1376 de 1958.

13. La paternité de cette proposition est à porter au crédit de M. Géraud Fustier, propriétaire du domaine de Jalionas. 


\section{Chapitre 3. Le dépôt funéraire}

1 Les relations de la découverte archéologique à l'origine de la collection de Verna (fig. 12) conduisent à conclure qu'on a affaire à la fouille d'une sépulture gauloise. La question se pose de savoir si les mobiliers attribués à cette découverte et présentés comme un ensemble corroborent une telle interprétation. En effet, en Gaule, à la fin de l'âge du Fer et au début du Haut-Empire, plusieurs découvertes d'aspect funéraire (chambre boisée, important dépôt de vaisselle, armes etc.) se caractérisent par l'absence de tout reste osseux humain (Boulestin, Buisson, Gomez de Soto 2002). Ces découvertes encore énigmatiques (cénotaphes, dépôts cultuels...) doivent être distinguées des ensembles dans lesquels le corps du défunt est encore présent avec des restes plus ou moins nombreux récupérés dans les cendres du bûcher.

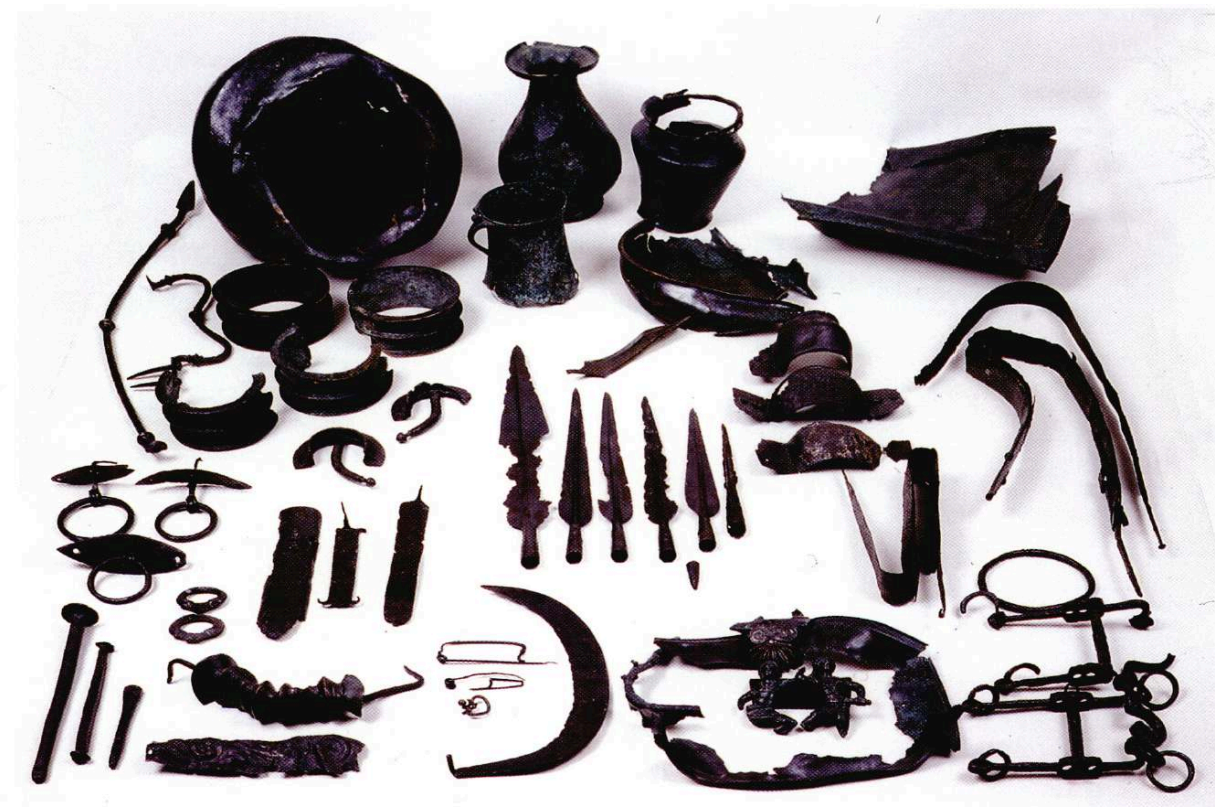

12 - Objets métalliques de la collection de Verna

2 A Verna, la présence d'ossements fut consignée dans les premiers comptes rendus de Fr. Artaud. Toutefois, ce dernier a écrit en marge du Ms 101 qu'il s'agissait "d'ossements d'animaux", "d'ossements" dans les autres versions. En revanche, l'auteur insiste sur la 
présence "parmi les ossements dont nous venons de parler... à fleur de terre d'ossements de chevaux répandant une odeur infecte, que nous fimes enterrer". Aucun reste humain ne fut alors signalé, mais les inventeurs ne disposaient sans doute pas des connaissances permettant de les identifier dans un contexte de crémation. Par chance, dans la collection de Verna figurent plusieurs esquilles et fragments d'os qui offrent l'opportunité de valider l'interprétation ancienne de "sépulture" et de confirmer la présence de faune.

\section{OSSEMENTS HUMAINS ET ANIMAUX. Fr. Blaizot, D. Lalaï}

La collection de Verna conserve deux fragments osseux assurément humains :

- Un fragment d'un pariétal droit humain mature conservé au niveau de l'obélion (postérolatérale droit) de 6,5 sur 4,5 cm, porte le foramen pariétal droit, cassé sur la pars postica de la suture sagittale, ouverte. En vue exocrânienne, le fragment est légèrement altéré: l'os cortical est décoloré et a un aspect mousse; en vue endocrânienne, l'aspect est normal. L'épaisseur varie de 6,5 à $8 \mathrm{~mm}$.

- Un fragment de voûte crânienne humaine mature, correspondant à la région du bregma, de 4 sur $5 \mathrm{~cm}$, portant la pars bregmatica des sutures coronale et sagittale. L'oblitération des sutures est du stade 1 de Broca en vue exocrânienne et des stades 3 (coronale) et 4 (sagittale) en vue endocrânienne. L'épaisseur est de 5 à $6 \mathrm{~cm}$, l'aspect de l'os est sain sur ses deux faces.

Ces deux fragments ne sont pas brûlés, ni même chauffés. Il est difficile d'affirmer qu'ils appartiennent ou non au même sujet. L'aspect de surface en vue exocrânienne n'est pas le même, mais l'altération du pariétal peut être due aux conditions d'enfouissement, si le bloc crânio-facial reposait au contact du sol par sa partie postérosupérieure. L'état des sutures diffère, mais l'on sait que la coronale se synostose plus rapidement que la sagittale. Toutefois, bien que l'épaisseur soit généralement plus importante au niveau de l'obélion qu'au niveau du bregma, la différence d'épaisseur entre les deux fragments est ici notable. Enfin, les particules de terre, incrustées à la surface du diploë au niveau des cassures, diffèrent profondément. Celles que l'on retrouve sur le fragment de la région bregmatique sont de couleur brun-rouge et relativement argileuses, tandis que celles qui adhéraient encore au fragment postérolatéral du pariétal droit sont plus claires et plutôt sablonneuses. Après lavage, seules les particules du premier ont coloré le diploë. Il semblerait donc que ces deux fragments ne proviennent pas du même contexte, et nous émettrons ainsi des doutes qu'ils appartiennent au même sujet.

De plus, aux restes du bassin de bronze adhère un fragment de côte brûlé, conservé sur une longueur de $16 \mathrm{~mm}$ et une largeur de $6 \mathrm{~mm}$. Il se présente par sa face médullaire et a donc été cassé longitudinalement, ce qui correspond au mode de fragmentation classique des côtes durant la crémation. L'organisation des travécules, qui sont longs et parallèles, est compatible avec un fragment de côte humaine. La couleur grise de l'os, teint dans la masse, indique qu'il a été porté à une température proche de 550-560 (Bonucci, Graziani 1975).

6 La faune, quant à elle, fut sans doute mieux représentée que ne le laissent penser les quelques vestiges conservés. L'échantillon comprend cinq fragments d'os brûlés ou chauffés dont deux recollent. On identifie l'épiphyse proximale d'un tibia droit, 
achevée, mais non soudée appartenant à un porcin juvénile. Sa couleur blanche indique que l'os a été porté a une température de plus de $650^{\circ}$. Deux fragments qui collent entre eux appartiennent à un métatarsien gauche d'équidé. Il est conservé sur une longueur de $7 \mathrm{~cm}$, son diamètre est de $32 \mathrm{~mm}$ environ. Cet os a été chauffé, comme l'indique sa couleur qui varie du brun foncé au noir (température portée de $250-300^{\circ}$ à $350^{\circ}$ ). Un fragment de diaphyse du même type équidé que le précédent est conservé sur une longueur de $3,5 \mathrm{~cm}$. Sa couleur varie du gris au blanc (température portée de $550^{\circ}$ à plus de $650^{\circ}$ ). Enfin, on recense encore un fragment de diaphyse indéterminée, conservé sur une longueur de $3 \mathrm{~cm}$ est de couleur noire (température portée à $\left.300-350^{\circ}\right)$.

\section{L'ÉTAT DE CONSERVATION DU MOBILIER MÉTALLIQUE. B. Herbold}

7 Le mobilier de Verna a été trouvé à une époque où les fouilles archéologiques n'étaient pas vraiment scientifiques. Cependant, lors de la découverte, les grands et les beaux objets n'ont pas été les seuls ramassés ; l'intérêt s'est également porté sur de petits fragments, qui ne permettent pas toujours d'identifier les objets : les fragments de tôle de bronze et les gouttes de bronze fondu par exemple. Malgré cela, certains éléments manquent et d'autres ont pu être négligés complètement.

8 La description des circonstances de la trouvaille de 1818 indique quelques détails sur l'incinération de la tombe principale: "Un lit de charbon et d'ossements en partie brûlés, d'un pied et demi d'épaisseur, sur six en carré". L'état de conservation des objets, qui étaient dans le feu, a été aussi remarqué : "le feu même a du être si violent, que l'on a trouvé quelques parties des ornements du char et de l'armure entièrement fondues". On a aussi noté le pliage antique des épées: "à cette observation, on peut joindre celle, que les trois sabres que cette fouille a produite, étaient tous repliés en trois parties [...]" (cf. annexe 1).

9 Tout les éléments en fer portent des traces de feu qui se signalent par une couche d'oxydation particulière qui a très bien conservé les objets. La surface originelle pu être retrouvée par micro-sablage (Berducou $1990: 198-199$ ). Aucune trace de tissus ou de restes organiques n'a été observée.

10 L'inventeur a essayé de détordre quelques objets en bronze et en fer, comme les épées, après la découverte en 1818. Cette action se remarque grâce à l'éclatement de la couche d'oxydation et par la mise à nu du noyau métallique. Aujourd'hui, il est possible de bien discerner ces manipulations modernes, des pliages et des dégradations antiques, qui ont fait partie des rites funéraires.

11 La surface de quelques objets en fer montre des tâches rouges éclatantes, qui ont été identifiées par Mme Dr. S. Greiff (RGZM) comme de l'oxyde de fer en forme d'hématite. La présence d'une couche d'hématite est la cause d'un rougissement des objets en atmosphère oxydante. Cette méthode est un moyen de conservation très simple et très ancien qui laisse éclater la couche de crasses (Rosenberg 1917; Scharff et al. 2000 : 64-65). La couche d'hématite se trouve sur la surface conservée par l'incinération antique ; des couches d'oxydation ont pu parfois réapparaitre.

12 Les objets en bronze sont tous très bien conservés, lorsqu'ils n'ont pas été fondus par le feu de l'incinération. La grande situle de type Beaucaire ( $c f$. infra) était le seul objet 
couvert par une couche noire d'oxydation. Les couches d'oxydation ont été enlevées par grattage avec des outils appropriés.

\section{LES PARURES VESTIMENTAIRES. J.-P. Guillaumet, M. Schönfelder}

\section{LES FIBULES}

Le mobilier de Verna comprend plusieurs fragments de fibules en fer qui ne figurent pas sur les planches anciennes - témoignant du peu d'attention qui leur était alors accordée - à l'exception d'un exemplaire, sans doute du type de Nauheim (fig. 5, objet $\mathbf{n}^{\circ}$ VI). L'état de conservation de ces fibules, avec une patine noire caractérisant les objets passés au feu correspond bien aux autres objets de la tombe à char.

\section{Inventaire :}

- Une grande fibule de schéma La Tène moyenne est conservée en deux parties (arc et ardillon); l'arc filiforme est en forme de U très allongé, avec le début du porte-ardillon (long, conservée 9,5 cm). Le fil formant ce dernier, d'un diamètre plus petit, se replie sur toute la longueur de l'arc et y est maintenu étroitement par deux bagues. L'une se situe vers le pied et la seconde s'appuie sur les spires du ressort. La seconde partie (long, conservée $13,2 \mathrm{~cm}$ ), l'ardillon, possède une spire d'un ressort à corde externe et à l'origine à trois spires. Le pied, ou porte - ardillon, non conservé, était rectangulaire (fig. 13, a).

- Une petite fibule de schéma La Tène moyenne ; l'arc filiforme est en forme de U très allongé, (long, conservée 3,1 cm). Le fil provenant du pied disparu, d'un diamètre plus petit, se replie sur toute la longueur de l'arc et y était maintenu étroitement par deux bagues. L'une, qui s'appuie sur les spires du ressort est conservée. Le ressort est à corde externe et à quatre spires. Un anneau (dia. 1,3 cm) passé dans le ressort indique qu'elle faisait partie d'une paire reliée par une chaînette (fig. 13, b). 


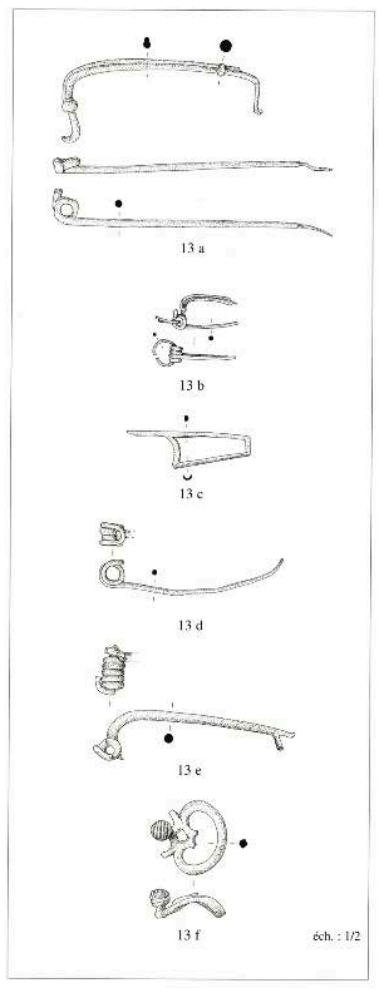

13 - Fibules et l'agrafe de ceinture

Les deux fibules appartiennent à un petit groupe peu courant et mal connu des fibules de schéma La Tène moyenne; elles pourraient en être d'un type évolué. Les comparaisons sont très rares et les contextes ne permettent qu'une datation peu précise : les oppida de Bibracte (dép. Saône-et-Loire/Nièvre), du Fossé des Pandours au Col de Saverne (dép. Bas-Rhin) et du Mont Terri (canton du Jura, $\mathrm{CH}$ ) ont donné des exemplaires comparables ${ }^{14}$ dans des contextes de la Tène $\mathrm{D} 1$. Une fibule, très proche a été trouvée dans la tombe 1192 de Wederath (Kr. Bernkastel-Wittlich, D), associée à une épée avec fourreau de type Ludwigshafen en fer (Haffner 1978: pl. 302, 1). Le fil formant le pied en partie disparu se replie sur toute la longueur de l'arc et y est maintenu étroitement par une bague, très proche des spires. Le ressort est aussi à corde externe et à quatre spires.

-Un porte-ardillon ouvert, rectangulaire, avec amorce de l'arc (long, conservée 5,8 cm); schéma La Tène finale (fig. 13, c).

-Un ardillon et ressort à quatre spires et corde interne avec départ de l'arc de section rectangulaire (long. 8,6 cm); schéma La Tène finale (fig. 13, d).

18 Ces fragments de fibules sont des types classiques de La Tène finale; toutefois leur taille ne permet pas de donner des précisions sur les types et sur les datations.

19 -Une fibule à ressort à dix spires, à corde externe, arc facetté et départ du porteardillon ouvert rectangulaire (long, conservée 9,3 cm); schéma La Tène finale (fig. 13, e).

Le type de cette dernière fibule est bien connu à Bibracte par plusieurs exemplaires (Guillaumet 1993 : pl. 5, 20-21). Leur construction filiforme et à corde externe, sans ergot ou griffe, en fait un type comparable aux constructions de la première série typologique de Bibracte datée de La Tène $\mathrm{Dl}$. Cette proposition de datation est 
confirmée par les travaux de $\mathrm{R}$ Gebhard sur les fibules de l'oppidum de Manching (Gebhard 1991: 22 pl. 47,738-740). Cette fibule appartient donc bien au dépôt de la tombe à char, daté de La Tène Dlb.

\section{L'AGRAFE DE CEINTURE}

21 Il s'agit d'un anneau réniforme en alliage à base cuivre (dia. max $3,4 \mathrm{~cm}$ ) avec deux pointes recourbées vers l'intérieur. En symétrie, un bouton émaillé (dia. $0,8 \mathrm{~cm}$, hauteur $1,3 \mathrm{~cm}$; pour une ceinture de $0,2 \mathrm{~cm}$ d'épaisseur) se trouve au milieu de deux "cornes" (fig. 13, f et fig. 90).

Ce type d'agrafe se rapproche du type $4 \mathrm{E}$ de la classification de G. Bataille ("agrafes à languette à appendices latéraux" "agrafes à ailettes" "geflügelte Ringgiirtelhaken") ${ }^{15}$, daté par l'auteur de La Tène D1-D2. L'agrafe de Verna forme, avec quelques autres, un groupe particulier à cause de son anneau portant des appendices dirigés vers l'intérieur de l'agrafe. Ce petit sous-type regroupe les agrafes de Nîmes/Chemin de la Ranquette (dép. Gard), de Saint-Marcel/Argentomagus (dép. Indre), de Mâlain (dép. Côte d'Or) et de Nanteuil-sur-Aisne (dép. Ardennes) ${ }^{16}$. L'agrafe de la tombe de Nîmes est dans un contexte bien daté de La Tène D1 confirmé par l'usage de l'émail caractéristique de cette époque.

\section{LA VAISSELLE EN BRONZE. M. Schönfelder, avec une contribution de A.-M. Adam}

23 L'ensemble de onze vases républicains en bronze, composé de types rares et de formes uniques, fait de Verna l'une des collections les plus importantes pour ces objets de prestige en Europe tempérée pour l'époque de La Tène. On recense une grande situle et deux plus petites, un gobelet, une cruche, deux patelles à manche, un bassin rectangulaire, un bassin à bec verseur, un couvercle et un fond de vaisselles inconnues, et enfin une pièce ancienne, un bassin étrusque, soit au moins onze pièces. 


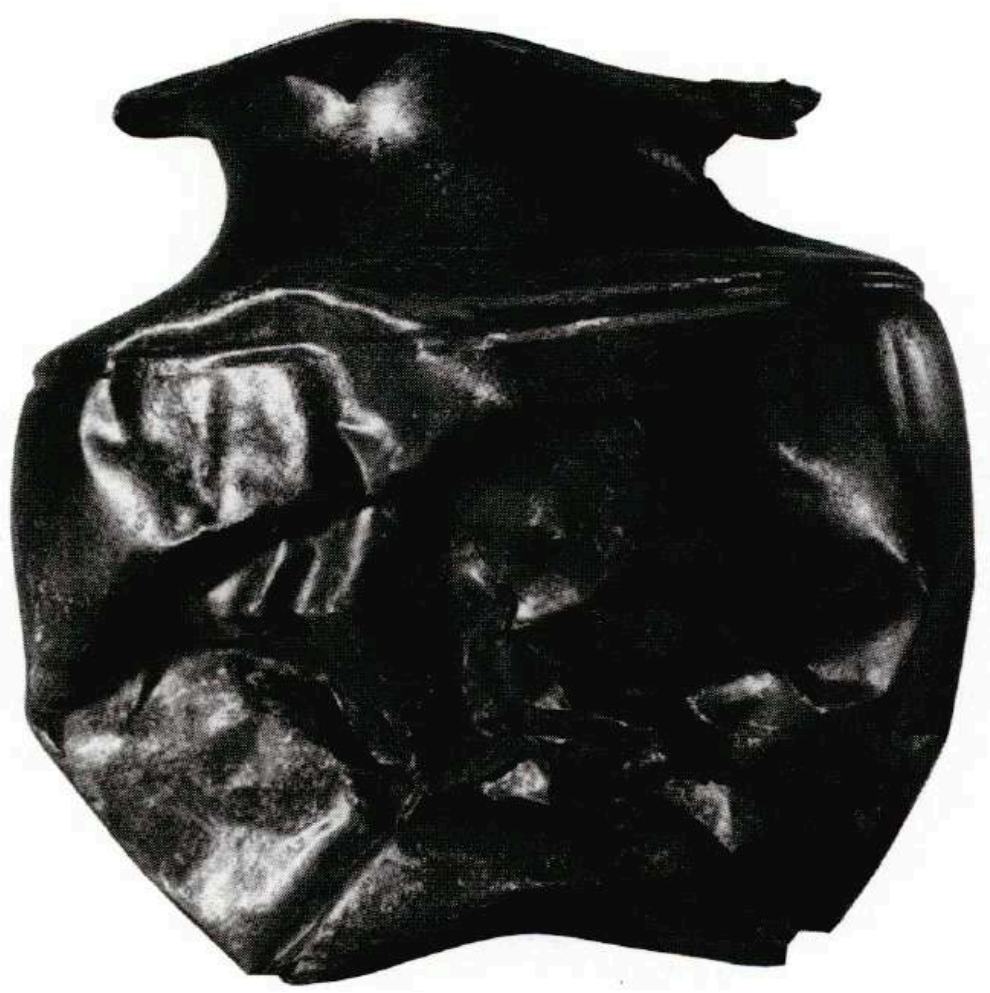

14 - Situle détériorée, $n^{\circ} 1$

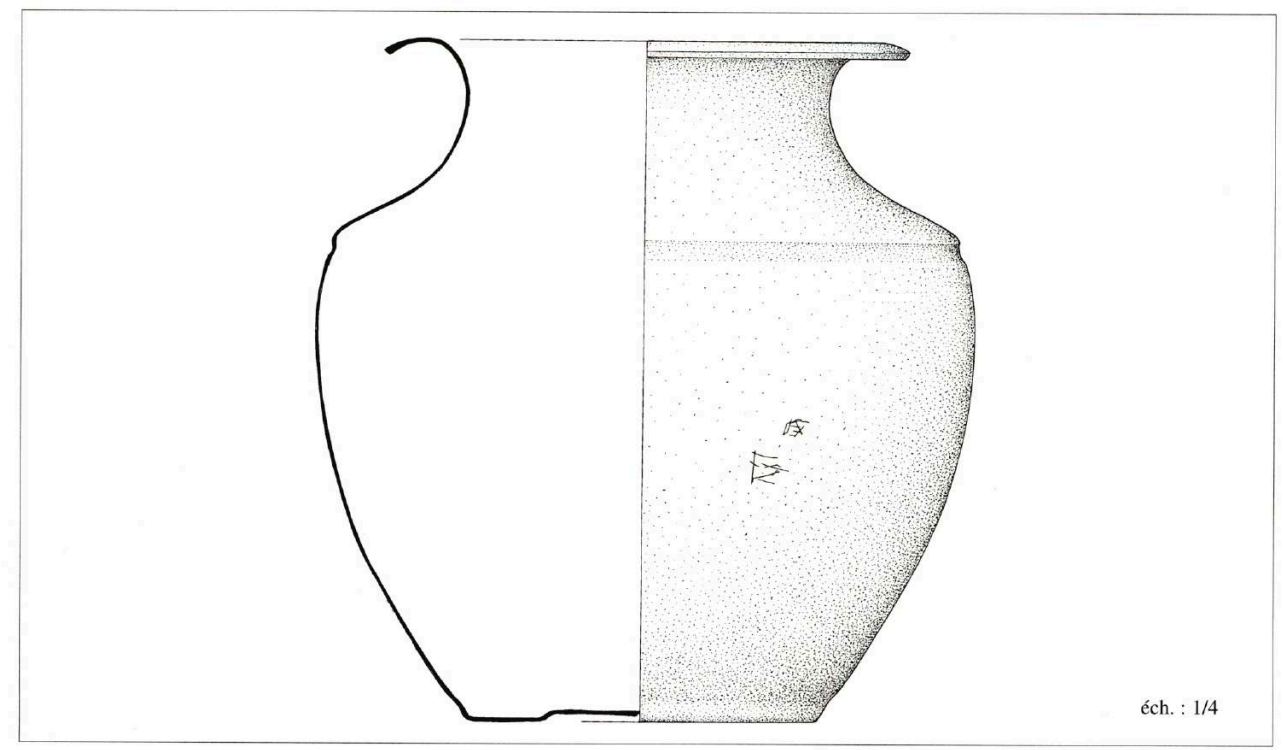

15-Situle de type Beaucaire (bronze), $n^{\circ} 1$

On peut clairement observer sur ces vases des traces de déformations anciennes. Des coups ont détérioré les parois de la situle de type Beaucaire (fig. 14) et de la cruche de type Kelheim. Les deux patelles à manche de type Aylesford ont des bords déchirés et leurs manches sont tordus. Quelques objets possèdent des surfaces avec des parties huileuses; ces stigmates montrent que les objets ont été partiellement brûlés. Toutes les attaches, les pieds et les éléments figuratifs comme les têtes de canards des patelles à manche, ont été détruits intentionnellement. Les cassures possédant la même patine que la surface des vaisselles, il faut alors considérer ces déformations comme faisant 
partie du rituel funéraire. Le bassin étrusque montre également les mêmes altérations avec en plus ses anses fortement brûlées, ce qui donne l'impression qu'il a été déposé en même temps que les autres vases; de plus, au XIXe s., on a tenté de les détordre, ce qui est reconnaissable aux éclats modernes visibles sur la surface de l'objet. Seule l'anse du gobelet de type Idria est relativement bien conservée, ainsi que deux des quatre pieds et certains fragments d'une anse du bassin rectangulaire. Les traces de destruction importante sur des vases en bronze sont exceptionnelles pour ces types d'objets qui sont généralement déposés intacts dans les tombes à incinération. Au cours de la restauration, des traces dues au feu, ont été également observées à la surface des vases. Avec ces destructions intentionnelles des bronzes, le rite funéraire de Verna fait exception à la règle des autres tombes de richesse comparable. De rares autres cas du Ier s. av. J.-C. sont connus à Acy-Romance (dép. Ardennes) ${ }^{17}$ et à Lamadelaine près de Titelberg $(\mathrm{L})^{18}$. Par contre, la dégradation des vaisselles métalliques est un rite plus courant à l'époque romaine, aux Ier et IIe s. ap. J.-C., comme à Ludwigshafen-Maudach (Kr. Ludwigshafen, D) ${ }^{19}$ ou dans la tombe 5 de Wölfersheim-Wohnbach (Wetteraukreis, D) ${ }^{20}$.

\section{SITULE DE TYPE BEAUCAIRE $\left(\mathrm{N}^{\circ} 1\right)$}

25 Parmi les vases en bronze de tradition italique, le plus grand est une situle (fig. 15) de type Beaucaire (H. originale $36,4 \mathrm{~cm}$, dia. max. $35,5 \mathrm{~cm})^{21}$ Si la plupart des exemplaires de ce type possèdent des pieds et des attaches pour les anses, celui de Verna n'en porte aucune trace. Cette pièce se distingue également par sa taille exceptionnelle (tab. 2). De plus, elle a été détruite par un petit nombre de coups, notamment des coups de pied et sa surface porte aussi de légères traces de feu ; enfin, la restauration a peut-être mis au jour des signes "gravés", qui ne sont pas vraiment identifiés ${ }^{22}$ (fig. 15).

26 Ce type de vaisselle est très rare, tant dans les habitats que dans les tombes. Dans la tombe 700 de Ehestorf-Vahrendorf (Kr. Harburg, D), une situle a été utilisée comme urne cinéraire; elle est datée de la fin de La Tène D1 grâce à deux fibules de type "geknickte Fibel" (Kostrzewski Var. K) (Wegewitz 1982: 43). Un autre exemplaire incomplet provient d'un terrain marécageux à côté de Fällanden (Canton de Zurich, $\mathrm{CH})^{23}$ Deux autres exemplaires, originaires de la tombe 19 de Beaucaire/Les Marronniers (dép. Gard), caractérisés par leurs attaches triangulaires avec des pointes foliacées, ont été décrits comme illustrant la variante A, par ailleurs bien connue. Les attaches plus larges en forme de feuilles de vigne, comme il en existe sur la situle de la tombe 12 de Beaucaire, définissent la variante B. Dans ces deux tombes, les situles sont accompagnées de simpula en bronze et de céramiques, qui permettent de dater cet ensemble entre 80 et 20 av. J.-C. (Fitzpatrick 1987: 102). Un fragment d'attache de Cáceres el Viejo (Prov. Extremadura, E) est daté avec un terminus ante quem de 80/77 av. J.-C.

27 A cause du petit nombre d'exemplaires connus, une datation exacte de la situle de Verna est difficile. Mais il est remarquable que ce type ne soit presque jamais exporté vers l'Europe centrale celtique. Les attaches caractéristiques, qui sont assez bien identifiées, sont absentes dans les habitats (Boube in : Feugère, Rolley [éd.] 1991: 19 sqq.). Ce type de situle était peut-être déjà remplacé à l'époque césarienne par d'autres formes (par exemple le type Eggers 18) alors que les importations de vases en bronze étaient plus importantes. Ainsi, la forme Eggers 18 est très fréquente dans l'Est de 
l'Europe centrale ${ }^{24}$ De plus d'autres grandes attaches, comme celles de Manching (Lkr. Pfaffenhofen a. d. Ilm, D) et de Podmokly (Okr. Rokycany, CZ) (Van Endert 1981 : 81), nous laissent penser qu'il existe d'autres formes de grands vases dont on ne connaît pas d'exemplaires complets.

Dans l'ensemble de Verna, il n'est pas possible d'assigner une fonction précise à cette grande situle de type Beaucaire. A. Fitzpatrick est très prudent en parlant de son utilisation dans le service à vin, et il propose comme hypothèse l'emploi des grandes situles avec les strigiles. Pourtant on ne connait à ce jour qu'une seule tombe avec cette association (Fitzpatrick 1987: $108 \mathrm{spp}$.). Dans le service à vin, plusieurs types de vases sont utilisés, pour l'eau, le vin et leur mélange, mais ces vases n'ont pas encore été identifiés. Peut-être peut-on regarder les grandes situles comme des récipients à eau ? Elles ne sont pas forcément nécessaires au service du vin lors du rite funéraire, les petites situles pour le vin étant suffisantes ; cela explique peut-être le petit nombre des grandes situles (à eau) dans les tombes, la petite situle à vin caractérisant à elle seule le rite.

\section{PETITES SITULES À ATTACHES D'ANSES. EN FER ( $\left.{ }^{\circ} 2-3\right)$}

Outre la situle de type Beaucaire, existent deux petites situles à attaches d'anses en fer. L'une est entièrement conservée (fig. 16) ; elle a une épaule arrondie, un corps droit et une partie inférieure conique (H. reconstituée $16,5 \mathrm{~cm}$, dia. de l'ouverture reconstituée $14,5 \mathrm{~cm}$, dia. du fond 10,4 cm). Les attaches en forme de deux triangles, joints par une passerelle, sont fixées au col par deux rivets. Des attaches similaires se trouvent à Ciringhelli (Prov. Verona, I) et à Wiebendorf (Kr. Hagenow, D) ${ }^{25}$ La forme précise n'est pas figurée dans le catalogue de H.-J. Eggers, mais on classe tout de même ces vases dans l'ensemble des situles de types 21-23, car les parties terminales des attaches sont plus élaborées, seul critère qui les distingue des formes standard.

Un autre fragment (fig. 16 b) de col incomplet (dia. 13,6 cm) et une anse en fer peuvent être attribués à un autre groupe de vases, dans lequel les crochets des anses sont fixés dans un anneau en fer qui entoure tout le $\mathrm{col}^{26} \mathrm{Ce}$ genre de dispositif se retrouve sur des formes de panses complètement différentes ${ }^{27}$. Les exemplaires avec des panses ovoïdes de Montefortino (Prov. Áscoli Piceno, I) et de Zubowice (Woi. Zamość, PL) sont datés des IIIe et IIe s. av. J.-C. (Brizio 1900, pl. 4,21). Des pièces avec un corps droit et une épaule courbée ou arrondie de Villanueva de Córdoba, (Prov. Córdoba, E) ${ }^{28}$ ou des 
"puits rituels" des environs de Toulouse ${ }^{29}$ proviennent de complexes datés après la fin du IIe ou au début du Ier s. av. J.-C.

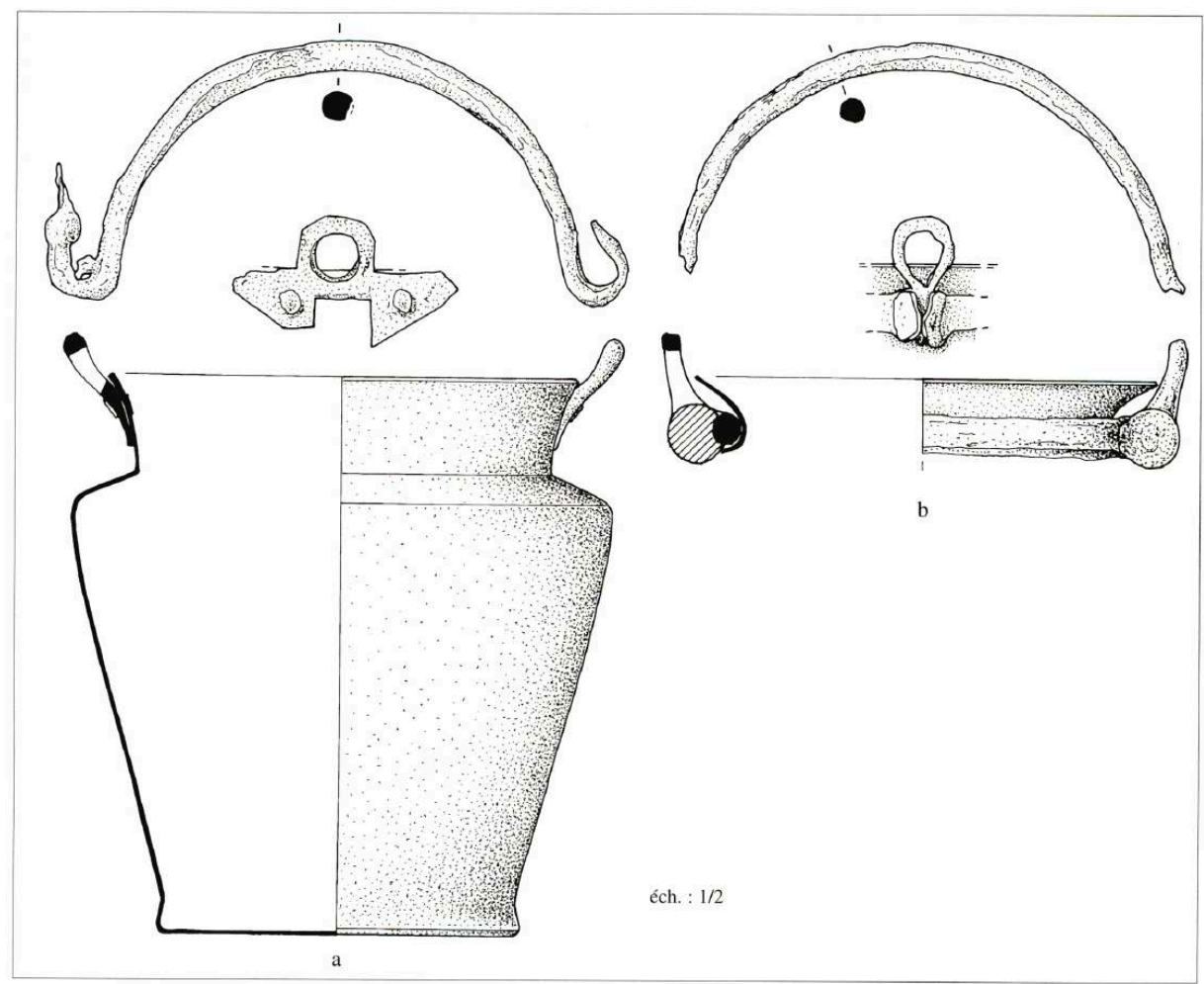

16-Situles (bronze et fer), $n^{\circ} 2$ et 3

31 La typologie des petites situles en bronze fondée sur les descriptions existantes ne peut donc pas être considérée comme satisfaisante. Si on observe les vases entiers, l'impression qui domine est celle d'une absence de types propres, avec au contraire des combinaisons multiples entre différents critères de formes d'anses et de corps. Pour ces vaisselles au repoussé, il existe également plusieurs critères pour les épaules (courbées/ arrondies), les corps (droits/ovoïdes) et les parties inférieures (coniques/rentrées) (tab. 3). Les variantes de cols et de fonds semblent n'avoir aucune importance pour le type. De plus, des yeux sont attachés de manières différentes pour les crochets des anses, avec de petites ou grandes attaches à noeuds et avec un ou deux rivets. Les combinaisons entre la forme des attaches et celle des panses ne nous semblent pas assez fréquentes pour pouvoir définir des types. Une attribution de variantes n'est donc pas nécessaire.

Nous proposons une description standardisée (tab. 3) ou une appellation générale comme "situle de type Eggers 21-23". Dans sa carte de répartition, H.-J. Eggers luimême n'a pas fait de différence entre ses types et il a parlé de "seaux en bronze en forme de situles à attaches en fer" (Eggers 1951 :161 suppl. 11). Au vu de la multitude de combinaisons (tab. 4), on peut conclure que les situles sont produites dans des ateliers différents, qui n'ont pas eu besoin de suivre des règles uniformes. L'idée d'une fabrication standardisée n'existait donc ni pour le fabricant, ni pour l'utilisateur. 


\begin{tabular}{|c|c|c|}
\hline Lieu de découverte & $\begin{array}{c}\text { Hauteur, } \\
\text { sans pieds }(\mathrm{cm})\end{array}$ & Bibliographie \\
\hline Verna (Dép. Isère, F) & 36,4 & \\
\hline Ehestorf-Vahrendorf, tombe 700 (Kr. Harburg, D) & 30 & Wegewitz $1982: 41$ \\
\hline Fällanden (Kt. Zürich, CH) & 27,2 & Wegewitz $1982: 47$ \\
\hline Beaucaire/Les Marronniers, tombe 19 situla n 1 (Dép. Gard, F) & 26,1 & Dedet $1978: 107$ fig. $65,5$. \\
\hline Beaucaire/Les Marronniers, tombe 19 situla n 2 (Dép. Gard, F) & 25,8 & Dedet $1978: 107$ fig. 65,4 . \\
\hline Beaucaire/Les Marronniers, tombe 12 (situla var. B) (Dép. Gard, F) & 24 & Dedet $1978: 94$ fig. $55,2$. \\
\hline
\end{tabular}

Tableau 2 -Situles en bronze de type Beaucaire. Hauteur des vaisselles complètes

\begin{tabular}{|l|l|l|l|}
\hline \multicolumn{1}{|c|}{ Epaule } & \multicolumn{1}{|c|}{ Corps } & Partie inférieure & \multicolumn{1}{c|}{ Attache } \\
\hline arrondie & droit & conique & attache de deux triangles joints (2 rivets) \\
\hline courbée & ovoïde & rentrée & attache d'un triangle massif \\
\hline & & & attache à grand noeud à 4 rivets \\
\hline & & & attache à petit nœud à 2 rivets \\
\hline & & & manchette à cercle de fer \\
\hline & & & uniquement des trous de rivets conservés \\
\hline
\end{tabular}

Tableau 3 -Petites situles à attaches à anses en fer : liste des critères possibles

\begin{tabular}{|c|c|c|c|}
\hline Vaisselle & Forme des attaches & Rivets & Lieux de trouvaille \\
\hline $\begin{array}{l}\text { épaule arrondie / corps droit / } \\
\text { partie inférieure conique }\end{array}$ & $\begin{array}{l}\text { uniquement des trous } \\
\text { de rivets conservés }\end{array}$ & 2 & Idria, tombe 17 \\
\hline $\begin{array}{l}\text { épaule arrondie / corps droit / } \\
\text { partie inférieure conique }\end{array}$ & attache à grand nœud & 4 & $\begin{array}{l}\text { Idria, tombe } 2 \\
\text { Musée Chalon } n^{\circ} 48 \text { (seulement rivet) }\end{array}$ \\
\hline $\begin{array}{l}\text { épaule arrondie / corps droit / } \\
\text { partie inférieure rentrée }\end{array}$ & attache à petit næud & 2 & $\begin{array}{l}\text { Cavriana } \\
\text { Musée Chalon } n^{\circ} 47 \text { (nœud long, mais seulement } 2 \text { rivets) }\end{array}$ \\
\hline $\begin{array}{l}\text { épaule arrondie / corps droit / } \\
\text { partie inférieure rentrée }\end{array}$ & attache à grand nœud & 4 & Kappel \\
\hline $\begin{array}{l}\text { épaule arrondie / corps ovoïde / } \\
\text { partie inférieure conique }\end{array}$ & attache à un rivet & 1 & $\begin{array}{l}\text { Vieille-Toulouse } \\
\text { «puits funéraire » } 23, \mathrm{n}^{\circ} 313\end{array}$ \\
\hline $\begin{array}{l}\text { épaule arrondie / corps ovoïde / } \\
\text { partie inférieure rentrée }\end{array}$ & attache à petit nœud & 2 & Valeggio sul Mincio, tombe 4 \\
\hline $\begin{array}{l}\text { épaule courbée / corps droit / } \\
\text { partie inférieure conique }\end{array}$ & $\begin{array}{l}\text { uniquement des trous } \\
\text { de rivets conservés }\end{array}$ & 2 & $\begin{array}{l}\text { Slap ob Idrijci } \\
\text { Reka, tombe } 3 \text { (restes de fer) ; Viadana }\end{array}$ \\
\hline $\begin{array}{l}\text { épaule courbée / corps droit / } \\
\text { partie inférieure conique }\end{array}$ & attache d'une pièce & 2 & Wiebendorf, tombe 99 \\
\hline $\begin{array}{l}\text { épaule courbée / corps droit / } \\
\text { partie inférieure conique }\end{array}$ & attache à grand nœud & 4 & Musée Chalon $\mathrm{n}^{\circ} 49$, (seulement des trous de rivets) \\
\hline $\begin{array}{l}\text { épaule courbée / corps droit / } \\
\text { partie inférieure rentrée }\end{array}$ & $\begin{array}{l}\text { uniquement des trous } \\
\text { de rivets conservés }\end{array}$ & 2 & Goeblingen-Nospelt, tombe A \\
\hline $\begin{array}{l}\text { épaule courbée / corps droit / } \\
\text { partie inférieure rentrée }\end{array}$ & attache à grand nœud & 4 & Wiebendorf, tombe 1 \\
\hline
\end{tabular}

Tableau 4 -Petites situles à attaches à anses en fer : liste des vaisselles complètes (Bolla In :Feugère, Rolley (éd.) 1991)

\section{GOBELET À ANSE DE TYPE IDRIA (N4)}

Un gobelet à anse de type Idria ${ }^{30}$ est quasi complet (fig. 17); il ne manque que le fond et il ne présente pas de déformations importantes. Une marque est due à un coup de pioche récent. Le fond a été probablement perdu lors de la récupération de l'objet en 1818 car, dans les vaisselles battues, le passage entre celui-ci et le corps est habituellement très fin et très sensible à la corrosion, qui ici a rongé ses bords. Sur d'autres vases, on peut parfois observer des réparations antiques $d u$ fond $^{31}$. L'anse soudée a été arrachée et détériorée délibérément. Avec une hauteur de $13 \mathrm{~cm}$, ce gobelet est le plus grand appartenant à ce type (tab. 5), car la plupart des autres exemplaires mesurent entre 10 et $11 \mathrm{~cm}$. 
La fonction de ces gobelets a suscité plusieurs interprétations : ils furent tout d'abord interprétés comme des gobelets à boire. $\mathrm{M}$. Feugère, quant à lui, a proposé une fonction liée aux ablutions en contexte de symposium (Feugère, Rolley [éd.] 1991 :54) et enfin J. Metzler a proposé une utilisation avec des passoires, à cause de leurs diamètres semblables. Il conçoit un modèle où l'on puise le vin avec le simpulum, on le verse à travers la passoire dans le gobelet, pour ensuite le reverser dans d'autres récipients à boire (Metzler 1995 : 332).

On a probablement plutôt bu dans des gobelets hémisphériques (Raddatz 1969 : 86 sqq. ; Graue 1974: 29 sqq.) que dans des gobelets type Idria, si toutefois on a utilisé des récipients métalliques. Pour les ablutions rituelles, l'utilisation des cruches de type Kelheim est plus fréquente que celle des gobelets. Dans les tombes avec patelles d'ablution (tab. 7) d'Italie du Nord, les cruches ont peut-être également été remplacées par des gobelets à anses. La fonction de ce type de vaisselle est peut-être multiple. Par contre, l'ensemble des tombes ne contient pas systématiquement des gobelets associés avec des patelles à manche ${ }^{32}$.

La représentation quantitative de chaque type est également significative : les passoires sont les formes de vaisselle en bronze les plus courantes en Europe celtique. On retrouve souvent leurs petits éléments faiblement soudés qui se détachent. S'ils sont plus rares que les passoires, les gobelets le sont moins que les cruches, les situles et les patelles à manche ${ }^{33}$.

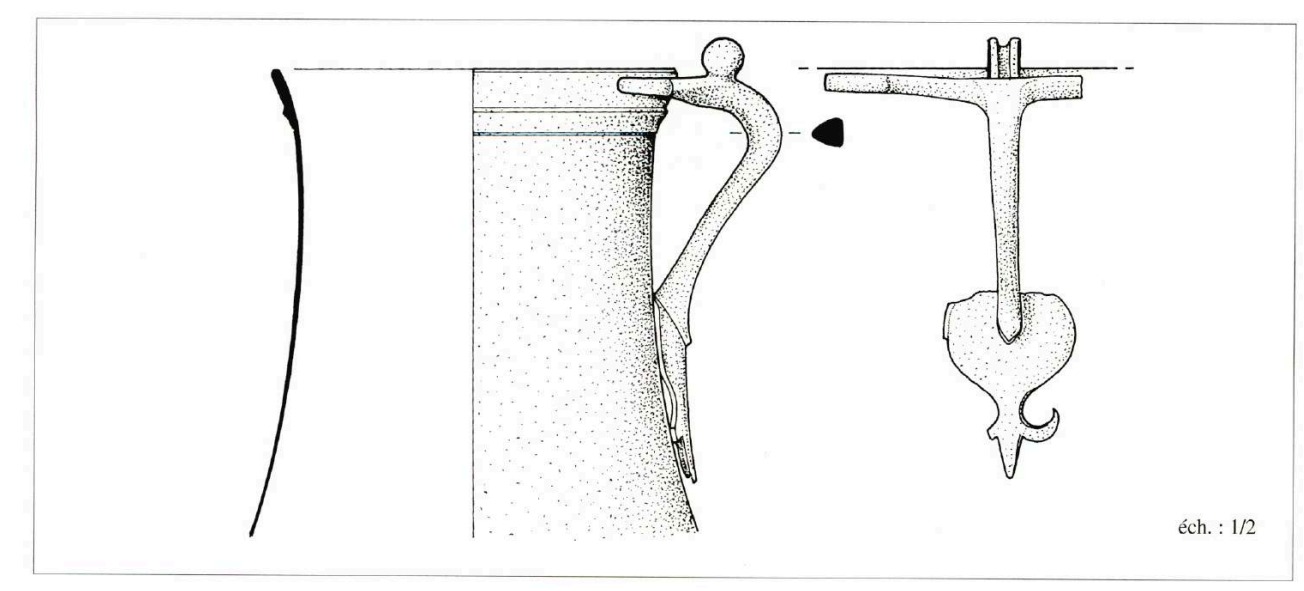

17-Gobelet à anse, de type Idria (bronze), $n^{\circ} 4$ 


\begin{tabular}{|c|c|c|c|c|}
\hline Lieu de découverte & $\begin{array}{c}\text { Hauteur } \\
(\mathrm{cm})\end{array}$ & $\begin{array}{l}\text { Dia. du } \\
\text { fond (cm) }\end{array}$ & $\begin{array}{l}\text { Dia. de } \\
\text { la levre (cm) }\end{array}$ & Bibliographie \\
\hline Verma (Dép. Isère. F) & 13 & 12,8 & 11,2 & \\
\hline $\begin{array}{l}\text { Ormavasso/San Bermardo, tombe } 15 \\
\text { (Prov, Novara, D) }\end{array}$ & 11,6 & 10.9 & 9,8 & Graue 1974, pl. 17.2. \\
\hline Delos (GR) & 11.2 & 10,9 & 9,4 & Feugère, Rolley (éd.) 1991 : 56 fig. $5,41 \mathrm{a}$ \\
\hline $\begin{array}{l}\text { Ornavasso/San Bernardo, tombe } 4 \\
\text { (Prov. Novara, I) }\end{array}$ & 11.2 & 11 & 10.4 & Grave 1974, pl. 5, 5. \\
\hline $\begin{array}{l}\text { Ornavasso/San Bernardo, tombe } 3 \\
\text { (Prov, Novara, I) }\end{array}$ & 11,1 & 10.8 & 10.5 & Graue 1974, pl. 3, 4 . \\
\hline $\begin{array}{l}\text { Valeggio sul Mincio, tombe } 4 \\
\text { (Prov, Verona, I) }\end{array}$ & 10,9 & 10,2 & 9,4 & Salzani $1995: 14$ pl. $4,6$. \\
\hline $\begin{array}{l}\text { Omavasso/San Bermardo, tombe } 84 \\
\text { (Prov. Novara, I) }\end{array}$ & 10,9 & 10,3 & 9,1 & Graue 1974, pl. $30,5$. \\
\hline $\begin{array}{l}\text { Omavasso/San Bernardo, tombe } 130 \\
\text { (Prov. Novara, 1) }\end{array}$ & 10.9 & 11.6 & 9,6 & Graue 1974, pl. 33, 2. \\
\hline Volterra (Prov. Pisa, 1) & 10,8 & 11 & 9,6 & Feugère, Rolley (ed) $1991: 56$ fig. $5,31$. \\
\hline Povegliano $\mathrm{n}^{\circ} 1$ (Prov, Verona, I) & 10,6 & 10 & 9 & Salzani $1983 / 84: 366$ pl. 3, 7. \\
\hline Povegliano $n^{\circ} 2$ (Prov. Verona, 1 ) & 10,2 & 10.4 & 9 & Salzani $1983 / 84: 366$ pl. 3,8 \\
\hline Ciringhelli (Prov: Verona, I) & 10.5 & 10,4 & 9 & Salzani 1983/84: 370 pl. 7,2 . \\
\hline Casalandri. tombe 1981 (Prov. Verona, I) & 10.5 & 11 & 9.2 & Salzani 1998 : 93, pl. 2 A, 4. \\
\hline Casalandri, tombe 46 (Prov, Verona, I) & 10,5 & 11.1 & 9 & Salzani 1998 : 93 pl. 27 A, 2. \\
\hline Agde (Dép. Hérault, F) & 10,4 & 10,6 & 9,2 & Feugère $1989: 7$. \\
\hline Manching (Lkr. Pfaffenhoofen a. d. Ilm, D) & 10 & 10 & 9 & Van Endert 1991. pl. 25, no. 400. \\
\hline $\begin{array}{l}\text { Wederath, tombe } 142 \\
\text { (Kr. Bernkastel-Wittlich, D) }\end{array}$ & 8,6 & 7,9 & 7,3 & Haffner 1971, pl. $29,19$. \\
\hline Tristse (SLO) & 8.3 & 7.8 & 6.9 & Gusțin $1991: 69$ fig. $32,5$. \\
\hline Idria pri Baži, tombe 5 (SLO) & 8,1 & 8,6 & 7,6 & Gustin 1991 : 14, pl. 8, 3. \\
\hline
\end{tabular}

Tableau 5 -Gobelets de type Idria. Dimensions d'exemplaires complets

\begin{tabular}{|c|c|c|c|c|}
\hline Lien de découverte & $\begin{array}{l}\text { Hauteur } \\
(\mathrm{cm})\end{array}$ & $\begin{array}{l}\text { Dia. du } \\
\text { fond (cm) }\end{array}$ & $\begin{array}{c}\text { Dia. de } \\
\text { la lère (cm) }\end{array}$ & Bibliographie \\
\hline $\begin{array}{l}\text { Hannogne-Saint-Rémy } \\
\text { (Dép. Ardennes, F) }\end{array}$ & 23 & 12 & 11,3 & Boubc in Feugère, Rolley (éd) 1991 : 40. \\
\hline Châtcau-Porcien (Dép. Ardennes, F) & 23 & 11.7 & 13 & Boube in Feugère, Rolley (éd). 1991 : 40. \\
\hline Verma (IDép. Isère, $F$ ) & 22,9 & 11,1 & 12 & \\
\hline Sanzeno (Prov. Trento, I) & 22,2 & 13 & 12 & Boube in Feugc̀re, Rolley (éd.) 1991 : 40. \\
\hline $\begin{array}{l}\text { Trebenišstc/Trehenísko Kale, tumbe } 5 \\
\text { (MAK) }\end{array}$ & 22 & 10,7 & 10,7 & Boube in Feugère, Rolley (é.) 1991 : 40. \\
\hline $\begin{array}{l}\text { Toutlouse/Estarac, puits } 9 \\
\text { (Dép. Hautc-Garronne, F) }\end{array}$ & 21,7 & - & 12 & Boube in Fcugère, Rolley (éd) $1991: 40$. \\
\hline $\begin{array}{l}\text { Raitenbucher Forst (I.kr. Weiisenburg- } \\
\text { Gunzenhausen, D) }\end{array}$ & 21,5 & & & Römer $2000: 312$. \\
\hline Kappcl (Kr. Biberach, D) & 21,2 & 10,9 & 11,6 & Boubc in Feugère, Rolley (cd.) 1991 : 40. \\
\hline $\begin{array}{l}\text { Pomas-Rouffiac-d' Audc/La Lagaste } \\
\text { (Dép. Aude, F) }\end{array}$ & 20,5 & 11 & 12 & Boube in Feugère, Rolley (ćd). 1991 : 40. \\
\hline $\begin{array}{l}\text { Chấtillon-sur-Indre, tombc } \\
\text { (Dép. Indre, F) }\end{array}$ & 20 & 10 & 10 & Boube in Feugère, Rollcy (éd) $1991: 40$. \\
\hline $\begin{array}{l}\text { Eyguiùres } \\
\text { (D)epp. Bouchcs-du-Rhône, F) }\end{array}$ & 20 & 10 & 15 & Boubc in Feugère, Rolley (êd.) 1991 : 40. \\
\hline Alt Mülln (Kr. Herrogtum Lauenburg, D) & 19,9 & 12,1 & & Buube in Feugère, Rolley (čd.) 1991 : 40. \\
\hline $\begin{array}{l}\text { Pomas-Rouffiac-d' Aude/La Lagaste } \\
\text { (Dép. Aude, F) }\end{array}$ & 19,8 & 10,3 & 9,8 & Boube in Feugère, Rolley (ćd.) 1991 : 40. \\
\hline Kelheim (Lk. Ketheim, D) & 19,5 & 9 & 10,5 & Boube in Fevgìrr, Rolley (éd). 1991: 40. \\
\hline $\begin{array}{l}\text { Toulouseffstarac, puits } 8 \\
\text { (Dép. Haute-Garonne, F) }\end{array}$ & 19,3 & 10.4 & 10,6 & Boube in Fengère. Rolley (éd.) 1991: 40. \\
\hline Turbino Nosate (Prov. Milano, D) & 18,8 & 10 & 10 & Boube in Fcugère, Rolley (éd) 1991 : 40. \\
\hline Nosate (Pruv, Milano, I) & 17.9 & & 11 & Boube in Feugàre, Rolley (ed,) $1991: 40$. \\
\hline
\end{tabular}

Tableau 6 -Cruches de type Kelheim. Dimensions des vases entiers 


\section{CRUCHE DE TYPE KELHEIM ( $\left.{ }^{\circ} 5\right)$}

La cruche en bronze comporte une panse piriforme avec un centre de gravité bas ( $\mathrm{H}$. totale $22,9 \mathrm{~cm}$ ) et un bord redressé avec une lèvre verticale (fig. 18) ${ }^{34}$ Ses trois pieds, caractéristiques pour le type Kelheim, ont laissé des traces de soudure au fond (dia. 11,1 $\mathrm{cm}$ ). Des marques circulaires signalent une production à l'aide du tour, avec lequel le fond a été rectifié. Le tour a aussi aidé à obtenir la forme finale ainsi qu'à polir la surface de la panse. Le vase est dans un très bon état de conservation, mais la panse a reçu un coup oblique avec un objet émoussé. Une partie de la surface couverte de quelques petites bulles témoigne que la cruche a été partiellement exposée au feu. L'anse soudée de cette "aiguière à anse" qui figurait sur une planche du manuscrit d'Artaud (fig. 4, objet EE), n'existe plus.

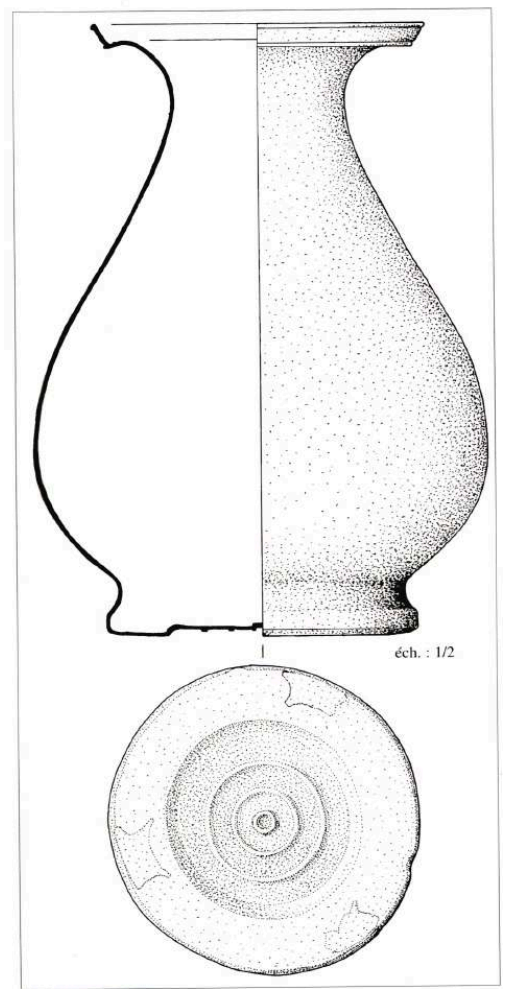

18-Cruche de type Kelheim (bronze), $n^{\circ} 5$

Les cruches de type Kelheim font partie des types classiques des vases importés en Europe centrale à La Tène $\mathrm{D} 1^{35} \mathrm{Si}$ des tombes et des dépôts ont livré des exemplaires complets, par contre, les habitats ne fournissent généralement que les pieds ou des fragments d'anses coulés en bronze massif ${ }^{36}$ Les dimensions de la cruche de Verna sont remarquables (tab. 6), comme celles du gobelet de type Idria et de la grande situle de type Beaucaire. Des vases semblables par leurs dimensions proviennent de la tombe de Hannogne-Saint-Rémy (dép. Ardennes), richement équipée avec des armes et un char, ainsi que de l'ensemble funéraire détruit de Château-Porcien (dép. Ardennes) (Flouest, Stead 1977 : 63-64, 69-72). 


\section{COUVERCLE À RIVETS ÉMAILLÉS ( $\left.N^{\circ} 6\right)$}

à bouton" se trouvent dans l'ancienne collection de Vieille-Toulouse (dép. HauteGaronne), sans que leur datation à La Tène finale soit assurée (Fouet, Savès 1971: 69, fig. 14, J. 14); un disque en bronze avec deux rivets fragmentés en fer, trouvé dans le camp romain de Numantia (Prov. Soria, E), peut aussi, être interprété comme un couvercle $^{39}$

Le couvercle se compose d'un disque en bronze avec des cercles concentriques (dia. 10 $\mathrm{cm}$ ) et d'un élément appliqué par trois rivets émaillés (fig. 19). Seuls les dessins de 1818 et de 1994/96 en témoignent car aujourd'hui l'objet n'est plus à Hières-sur-Amby. Sur la planche III (fig. 4, objet EE) le couvercle est figuré sur la cruche de type Kelheim. On ne peut dire si cette association doit être considérée comme un montage, ou si elle est fondée; la cruche ayant perdu son anse. On note toutefois que les diamètres de la cruche et du couvercle concordent.

Le disque rappelle les couvercles de vases en bronze qui sont bien connus à partir de l'époque augustéenne. Ces "couvercles à bouton" sont décrits pour la première fois dans la publication du camp légionnaire augustéen de Haltern (Kr. Recklinghausen, D) (Millier 1997 : 25, fig. 16-17). Les formes entières des vaisselles concernées sont connues uniquement par des découvertes dans des tombes, comme la tombe B de GoeblingenNospelt (L) ${ }^{37}$ ou celle d'Antran (dép. Vienne) (Pautreau 1991; Pautreau 1999: 38 sq.). Par son diamètre, le couvercle de Verna est très proche de l'exemplaire d'Antran, qui est associé à une forme plutôt trapue à col large. L'exemplaire de Goeblingen-Nospelt ressemble à une bouteille à col effilé. A Verna, aucun fragment de bronze (attaches massives ou fragments de panse) ne peut être attribué à un vase - dont la forme ne peut être déterminée - associé au couvercle. Les fouilles faites à Yverdon (canton de Vaud, $\mathrm{CH})$ ont livré, sans contexte daté, le fragment d'une anse de style républicain à charnière ${ }^{38}$ qui ouvre d'autres pistes.

Dans les habitats laténiens, les couvercles sont très rares : deux fragments de "manches $\mathrm{Au} v \mathrm{vu}$ du petit nombre d'objets connus, une datation précise de l'exemplaire de Verna n'est pas possible par comparaison avec d'autres vases à couvercles. Toutefois une datation à La Tène finale est assurée par la fixation de la charnière elle-même ; elle est réalisée à l'aide de trois rivets émaillés. Ces modifications appellent la remarque suivante: d'autres éléments de vases interprétés comme des anses laténiennes de cruches de type Kelheim sont fixés par des clous ou rivets émaillés aux vases (Feugère, Rolley [éd.] $1991: 129$ fig. 11). 


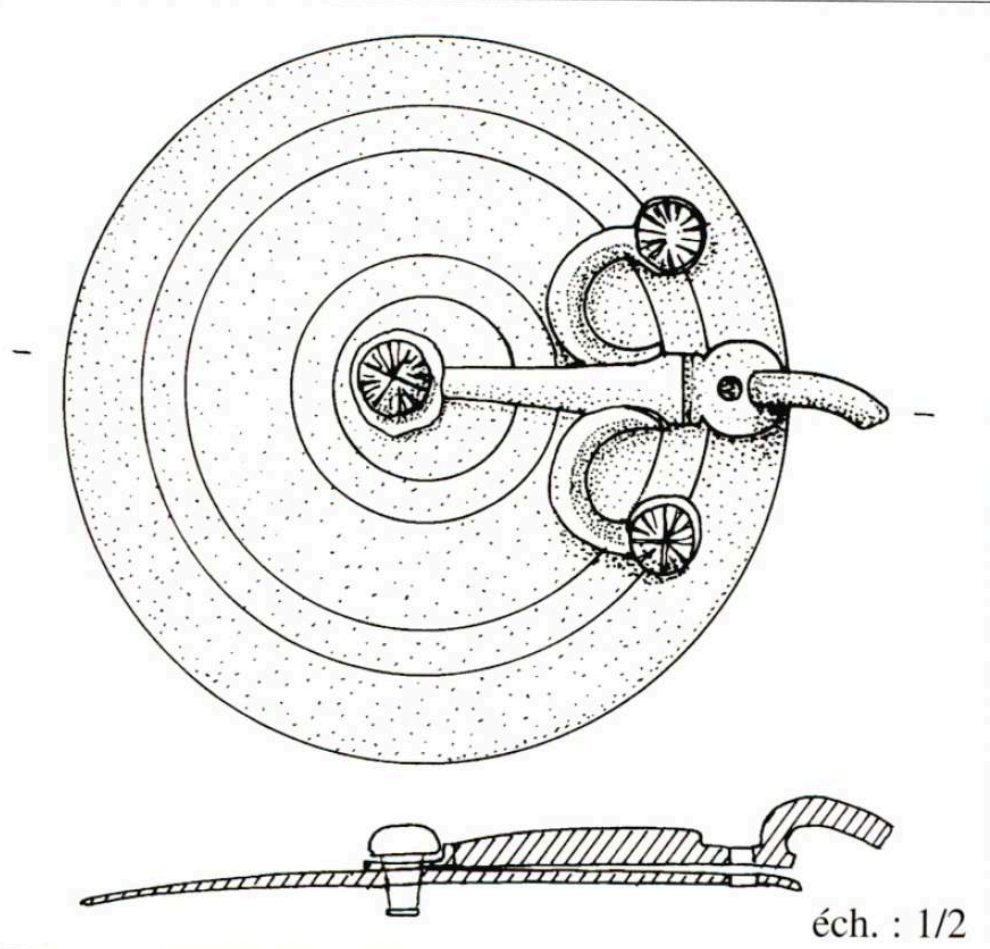

19 - Couvercle (bronze), n), $n^{\circ} 6$

\section{PATELLES À MANCHE DE TYPE AYLESFORD ( $\left.\mathrm{N}^{\circ} 7-8\right)$}

et pliés (fig. 20, $\mathbf{a}$ et b). Il manque la terminaison caractéristique des manches en forme de tête de canard, courante et souvent trouvée isolée dans les habitats. Ces objets semblent faire partie des rituels dans les habitats et nécropoles de l'époque. A Verna, un manche porte des incisions sur les côtés, interprétées comme des marques de fabrication ou de poids, que l'on retrouve par exemple à Saint-Germainmont (dép. Ardennes) (Feugère, De Marinis in :Feugère, Rolley [éd.] 1991 : 109, fig. 11).

Par leur diamètre $\left(\mathrm{n}^{\circ} 7: 27,5 \mathrm{~cm} ; \mathrm{n}^{\circ} 8\right.$ : environ $\left.26,6 \mathrm{~cm}\right)$ et leur hauteur $\left(\mathrm{n}^{\circ} 7: 6,7 \mathrm{~cm}\right)$ ainsi que par leur décoration sur le bord et sur les côtés des anses, les deux vases peuvent très bien être attribués au type Aylesford ${ }^{40}$ Leur fond est plat et sans trace de pieds soudés ; ils adoptent à plusieurs reprises la forme de coquillage ${ }^{41}$ et plus rarement celle, classique, de pelte comme par exemple ceux des tombes augustéennes précoces de Goeblingen-Nospelt, tombe B (L) et Gautzsch (Kr. Leipzig, D) ${ }^{42}$

patelle à manche et la cruche sont généralement considérées comme l'association classique du service des ablutions ${ }^{43}($ tab. 7). Peut-on pour autant douter de la provenance des deux patelles?

M. Feugère propose que des gobelets à anse de type Idria puissent aussi être utilisés pour des ablutions. Ainsi le gobelet remplace-t-il peut-être aussi la cruche dans le deuxième service de Verna, comme dans quelques autres (tab. 7). 


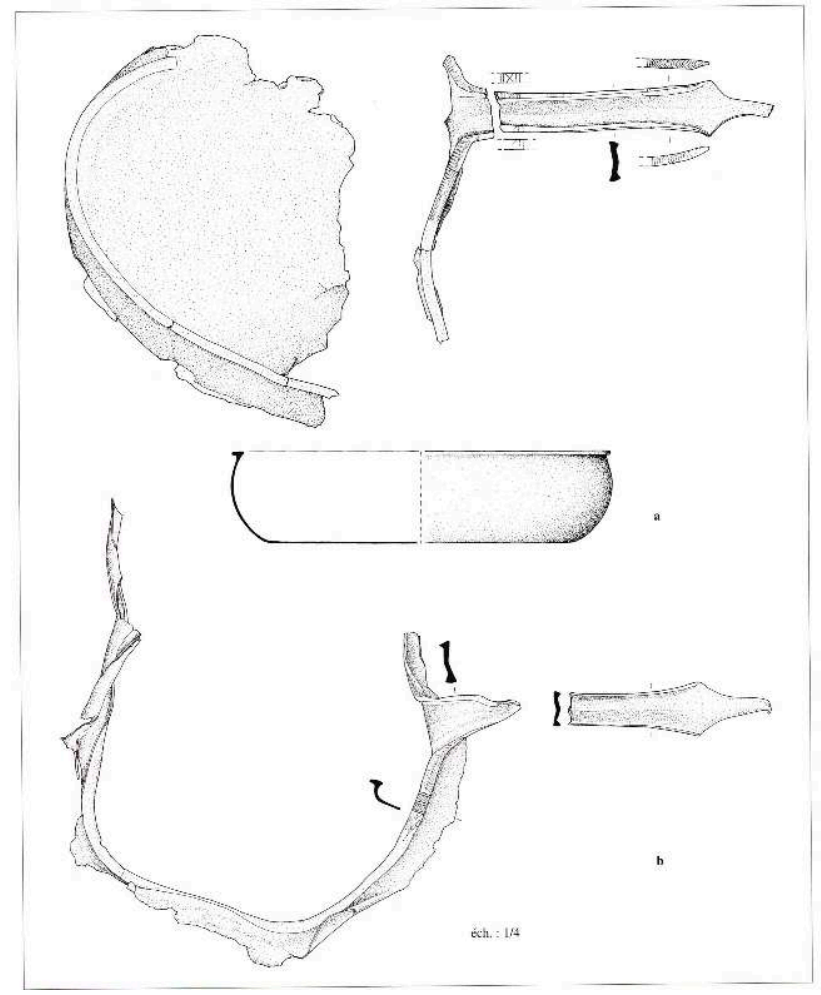

20-Patelles à manche type Aylesford (bronze), $n^{\circ} 7$ et 8 et restitution de la vasque

\begin{tabular}{|c|c|c|c|c|}
\hline Lieu de découverte & Patelle a manche & Cruche & Autres vaisselles métalliques & Biblliggraphie \\
\hline Dithren (Rhein-Neckar-Kreis, D) & type Muntefortino & type Piara Neamt & & Schunacher 19II. \\
\hline $\begin{array}{l}\text { Castiglione delle Stiviere } \\
\text { (Prow: Manova, I) }\end{array}$ & type Mantefortino & $\begin{array}{l}\text { cruche à anses a sece } \\
\text { des atraches en forme } \\
\text { de double dauphins }\end{array}$ & 22 gourdes, vase a pied & De Marinis 1997. \\
\hline $\begin{array}{l}\text { Isola Rines/Cusalandri, tombe } \\
46 \text { (Prux: Verona, D) }\end{array}$ & type Poręliano & & 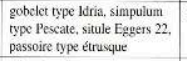 & Sultrani 1998, pl. 26-27. \\
\hline $\begin{array}{l}\text { Welwyn. lombe B } \\
\text { (Herttordshitr, GB) }\end{array}$ & Iypc Aylicsford & type Kelheim & 2 kantharoi en argent & Stead $1967: 58$ \\
\hline $\begin{array}{l}\text { Chátllion sur-Indre } \\
\text { (Dép. Indre. F) }\end{array}$ & Iype Aylesford & type Kelheim & Écuelle plate & $\begin{array}{l}\text { Ferditiref/illard 1993: } \\
\text { 96-107. }\end{array}$ \\
\hline $\begin{array}{l}\text { lannogne-Saint-Rémy } \\
\text { (Dép. Ardennes, F) }\end{array}$ & type Aylefford & type Kelheim & & Hlowest, Stead $1977:$ : 63-64. \\
\hline 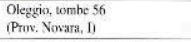 & type Aylesford & type Kelheim & & $\begin{array}{l}\text { Spragnolo Garzoli (div) } 1999 \\
118 \text { sq. fig. } 100 \text {. }\end{array}$ \\
\hline Gallarate (Prow. Varesce, I) & type Aylesford & type Gällarate & & $\begin{array}{l}\text { Feugerer, Rolley (ed.) } 1991 \text { : } \\
\text { 157. }\end{array}$ \\
\hline $\begin{array}{l}\text { Mezzano, tombe } 1 \\
\text { (Prow, Milano, 1) }\end{array}$ & type Aylesford & type Gallarate & & $\begin{array}{l}\text { Feugerec, Rolley (ed.).) } 1991 \text { : } \\
\text { 157? }\end{array}$ \\
\hline $\begin{array}{l}\text { Me/rano, trimbc } 2 \\
\text { (Prw. Milano, I) }\end{array}$ & type Aylesfond & type Gallaratc & & $\begin{array}{l}\text { Feugèr, Rolley (dd.) 1991: } \\
\text { 15?. }\end{array}$ \\
\hline $\begin{array}{l}\text { Valeggio "Le Buse" } \\
\text { (Prow. Verona, I) }\end{array}$ & type Aylesford & type Gallarate & simpulum type Pescate & $\begin{array}{l}\text { Feugtere, Rolley (ét) 1991: } \\
\text { 158. }\end{array}$ \\
\hline $\begin{array}{l}\text { Raillencourt Sainte-Olle } \\
\text { (Ditp. Nord, F) }\end{array}$ & Lype Aylefford & & bassin à bec verseur & Renseignement N. Ginoux. \\
\hline 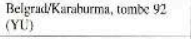 & type Aylesford & & simpulum type Pescate & $\begin{array}{l}\text { Tulorrvič 1972, pl. 28, } 14 . \\
\text { 15. }\end{array}$ \\
\hline $\begin{array}{l}\text { Acy-Rutuance, tumbe I, } 103 \mathrm{NM} \\
\text { (Dép.AAlemes, F) }\end{array}$ & type Aylestord & & & Larmbot 1998: 77 fig. 76. \\
\hline $\begin{array}{l}\text { Omavasaso "San Bemarda" ", } \\
\text { tombe } 3 \text { (Prov. Pedemante, I) }\end{array}$ & type Aylesford & & 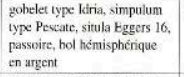 & Grave 1974, pl. 2-3. \\
\hline $\begin{array}{l}\text { Ornavasso" "San Bemado", } \\
\text { tombe } 130 \text { (Prov. Pedemome, I) }\end{array}$ & Lype Aylestorid & & 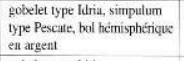 & Grauc 1974, pl. 33. \\
\hline Garlasco (Prow. Paviu, I) & type Aylesford & & guteelet type Idria & $\begin{array}{l}\text { Feugerere, Rolley (éd). 1991: } \\
\text { 158. }\end{array}$ \\
\hline 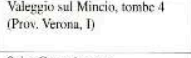 & type Aylesford & & $\begin{array}{l}\text { gobclet type Idria. simpulum } \\
\text { type Pescatic, situla Eggers } \\
21 \text {, pauscirc }\end{array}$ & Sal/ani 1995, pl. 4.5. \\
\hline $\begin{array}{l}\text { Saint Girmainmont } \\
\text { (Dép. Artenes, F) }\end{array}$ & Lype Aylesfind & type Oravasso-Ruvo & & $\begin{array}{l}\text { Fuggère, Rolley ( } 6 \mathrm{~d} \text { ) } 1991 \text { : } \\
109 \text { fig. } 11 .\end{array}$ \\
\hline $\begin{array}{l}\text { Ornarasso" "San Bermando", } \\
\text { lombe } 6 \text { (Prow. Pedemonte, i) }\end{array}$ & type Aylesford & type Omavasso-Ruvo & $\begin{array}{l}\text { simpulum type Pescate, } 2 . \\
\text { simpultum, situlat type Beacaire }\end{array}$ & Graue 1974, pl. 6-7. \\
\hline Aylessiond, tombe $Y$ (Kent, GB) & lype Aylesford & lype Kjacrumgarard & & Evans 1890. \\
\hline Guchlingen-Nospelt, tombe B (L) & $\begin{array}{l}\text { type Alesforid, } \\
\text { ave piects }\end{array}$ & type Kjaerumgard & $\begin{array}{l}\text { cruche avec couvertle, puisoir, } \\
\text { bassin, chaudirnn }\end{array}$ & Thill 1967. \\
\hline Gatusch (Kr. Leipzig, D) & $\begin{array}{l}\text { type Allestiond, } \\
\text { ave piect }\end{array}$ & & situla Eggers I8 & Eggers 1965 \\
\hline
\end{tabular}

Tableau 7 -Associations des vaisselles en bronze dans les tombes à patelle à manche (ensembles clos) 


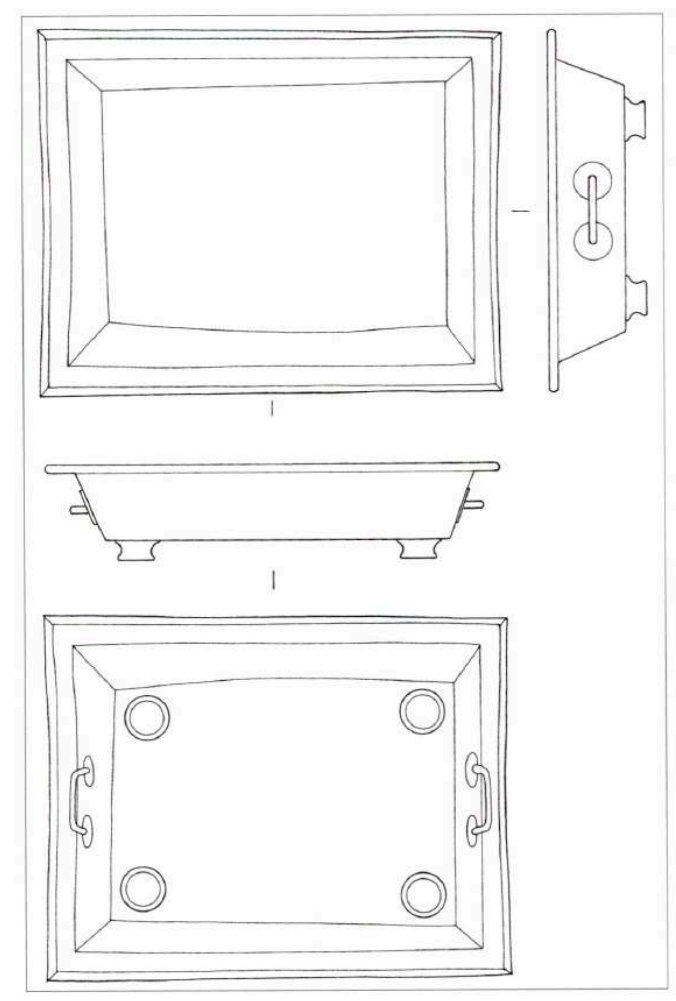

21-Reconstitution du bassin, $n^{\circ} 9$

\section{BASSIN RECTANGULAIRE ( $\left.{ }^{\circ} 9\right)$}

D'après plusieurs fragments de tôle en bronze, déchirés et pliés, il est possible de reconstituer un récipient en forme de bassin rectangulaire (fig. 21). Jusqu'à aujourd'hui, ce type reste unique. Le laboratoire de Mayence a beaucoup travaillé pour restituer sa forme: les fragments ont été copiés en résine artificielle, dépliés et assemblés par la restauratrice. Cela a permis de mettre en évidence un bassin avec un fond plat $(39 \mathrm{~cm} \times 26 / 27 \mathrm{~cm})$, un bord haut et droit avec une lèvre pliée qui s'insère dans un tube coulissé $(51 \mathrm{~cm} \mathrm{x} \mathrm{39/40,5} \mathrm{cm;} \mathrm{H.} \mathrm{10,2} \mathrm{cm;} \mathrm{dia.} \mathrm{tube} \mathrm{0,9} \mathrm{cm).} \mathrm{La} \mathrm{tôle} \mathrm{de}$ bronze est plus épaisse que celle des autres vases martelés.

$\mathrm{Au}$ fond, sont visibles les traces de soudure de quatre pieds ronds (dia. 4,8 cm), (fig. 22 $\mathbf{a}, \mathbf{b}, \mathbf{c})$, alors que des traces semblables se retrouvent sur les petits côtés à mi-hauteur (dia. 3,8 cm), provenant de la double fixation des anses. Au cours de la restauration, aucune trace de feu n'a été découverte à l'intérieur ou à l'extérieur du bassin. Deux des pieds sont conservés (dia. 4,8 cm, H. 2,1 cm), ils portent des traces importantes de feu et ont la forme d'une bobine. Des gouttes de bronze sont détachées de la surface. Les pieds sont ouverts d'un côté et fermés de l'autre. Un trou central sur le côté fermé indique un travail au tour. Par contre, les traces de soudure ne sont pas conservées, dues au feu secondaire. La moitié d'une anse brûlée se rapporte également au bassin (fig. $22 \mathrm{~d}$ ). De section ronde, elle est courbée et se termine par un disque par lequel elle était soudée au bassin. Ce montage des attaches trouve des parallèles dans la vaisselle hellénistique, comme par exemple le haut bassin en argent de la tombe royale de Es Soumâa du IIe s. av. J.-C., ainsi que dans d'autres vases en bronze ${ }^{44}$ Autre exemple, un vase étrusque du 
IVe s. av. J.-C., trouvé dans une tombe de La Tène finale à Langâ (Svendborg amt, DK) porte des anses de ce type (Sehested 1878, 174 pl. 37, b).

Le bassin de Verna reste sans parallèle. Par contre, on connaît des plateaux rectangulaires de l'époque augustéenne, par exemple dans le dépôt d'argenterie de Hildesheim (Kr. Hildesheim, D) (Gehrig 1981 : 32, fig. p. 33), mais ils sont moins hauts, sans anses et sans pieds soudés. Ils sont alors interprétés comme faisant partie de la vaisselle à manger. Pour l'époque républicaine, nous ne connaissons aucune forme rectangulaire ${ }^{45}$. Tous les vases en bronze sont martelés et en partie rectifiés au tour. Aucune imitation en céramique d'une forme analogue comme il en existe ensuite à l'époque impériale n'a été à ce jour mentionnée. Seule l'anse est un élément qu'on peut retrouver dans le monde méditerranéen, mais sa fabrication manque de finesse et de décor pour qu'on y voit un vrai produit de la toreutique de première qualité. Cependant, il ne faut pas écarter l'hypothèse d'une production en marge de la fabrication républicaine en masse des vases en bronze. Bien que les anses de Verna n'aient pas de parallèles dans les formes connues de vaisselle en bronze du Ier s. av. J.C., rien ne permet d'attribuer une datation ancienne au vase. De même il n'y a aucune raison pour une attribution à une production laténienne; sa provenance reste simplement inconnue.

La fonction de ce récipient n'est pas encore claire; au vu de sa hauteur, il convient de parler d'un bassin plus que d'un plateau. Nous n'avons pas trouvé de traces de feu à l'intérieur, indices d'un emploi comme brasero, ni aucune trace de découpe au couteau qui indiquerait un plat à servir.

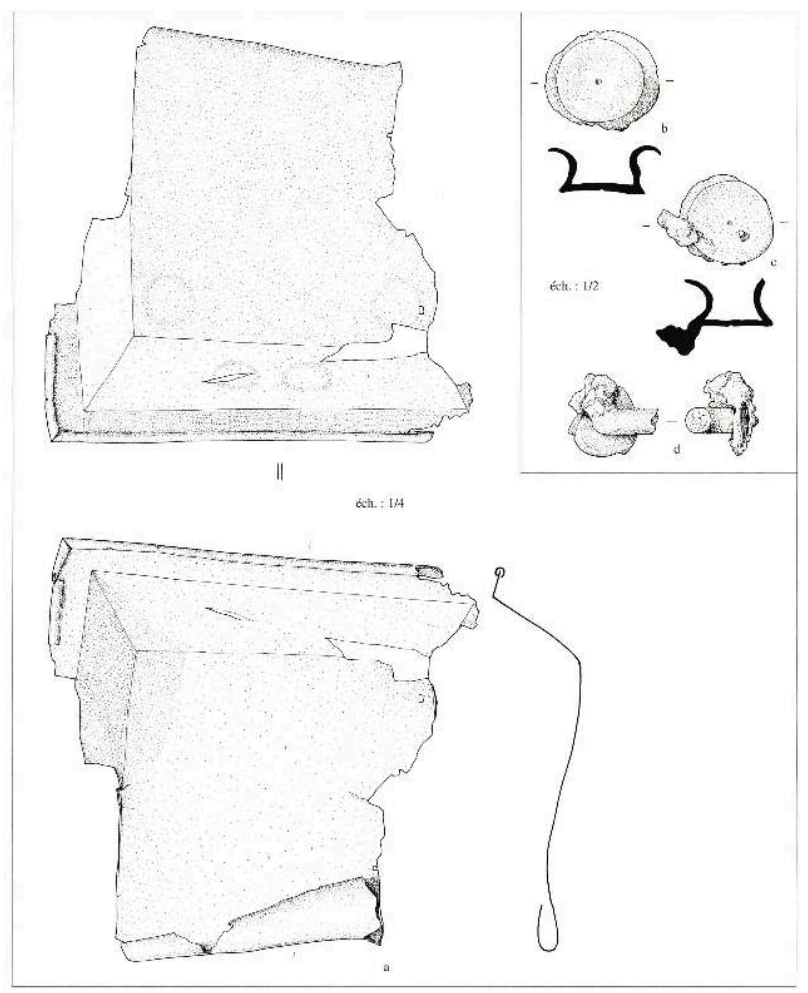

22 a - bassin rectangulaire (bronze), $n^{\circ} 9 ; b, c$ : pieds du bassin ; $d$ : anse du bassin 


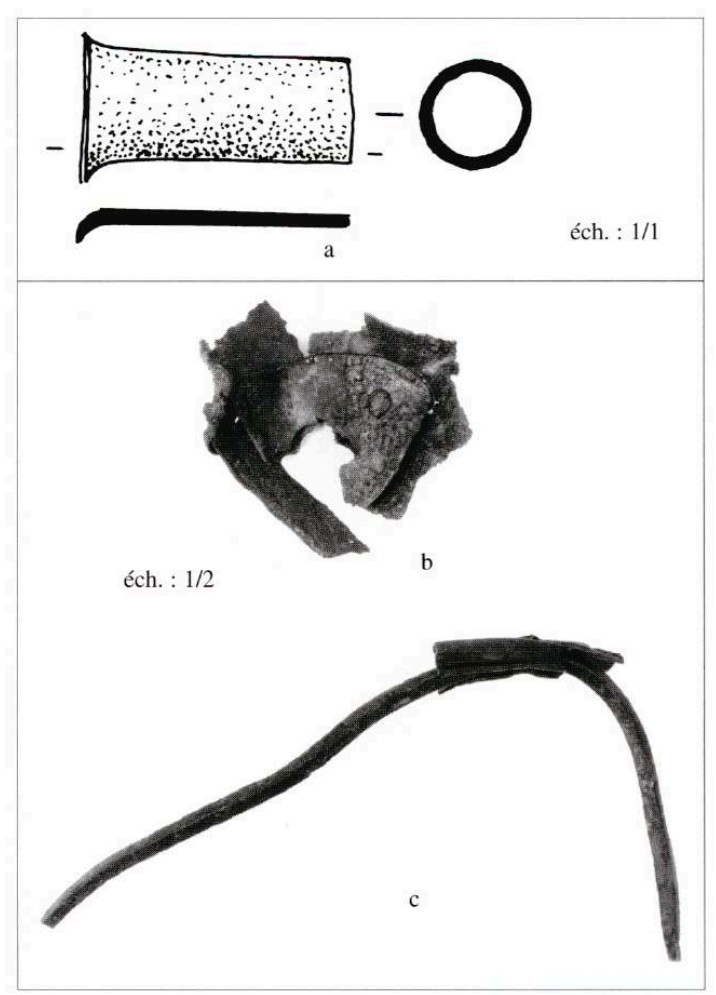

23 a -bec verseur (bronze), $n^{\circ} 10$; b et c : fragments du bord du bassin à bec verseur

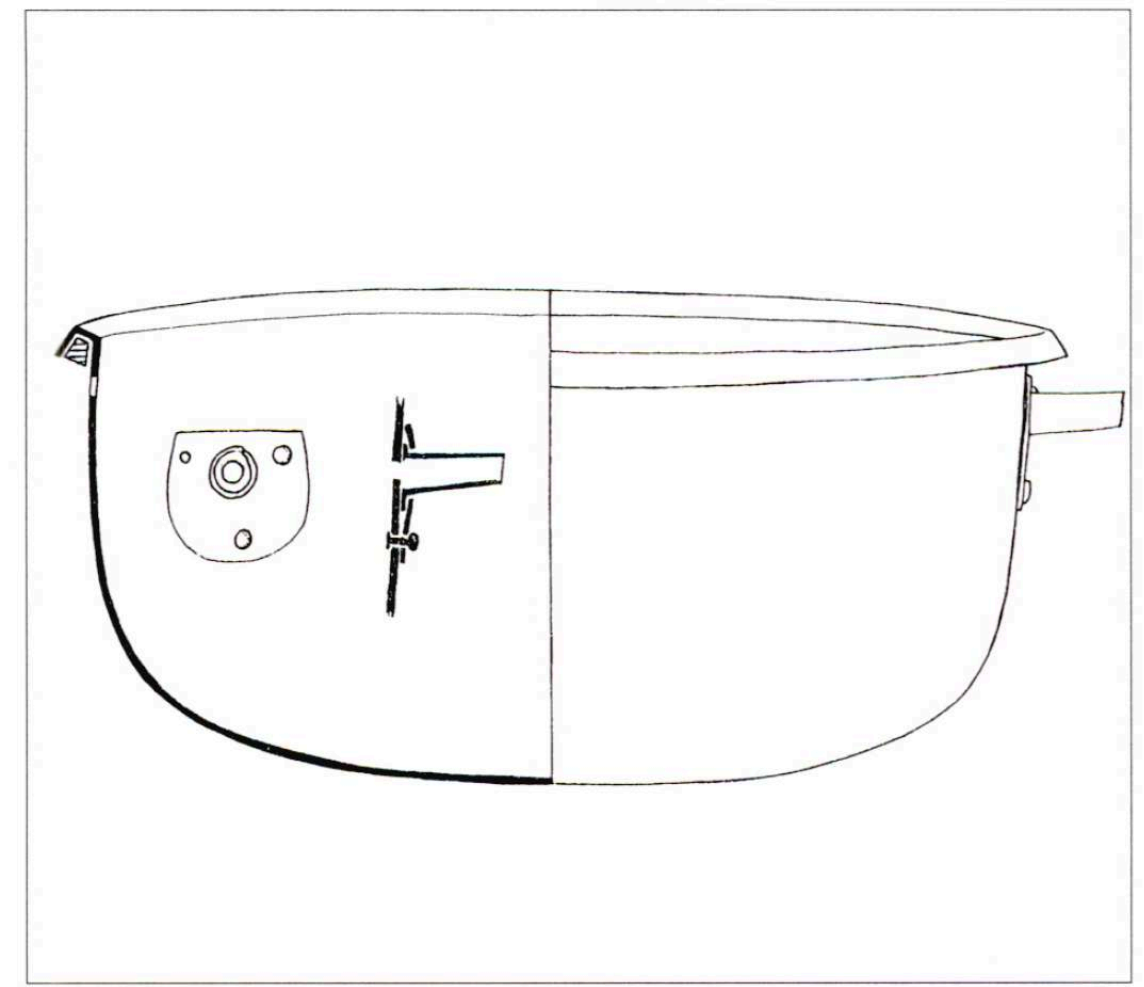

24-Wiebendorf, Kr. Hagenow, D, tombe 26 -Bassin à bec verseur (type Eggers 74), bronze et fer d'après Keiling 1984 : 8, pl. 6 


\section{BASSIN À BEC VERSEUR, DE TYPE EGGERS $73 / 74\left(\mathrm{~N}^{\circ} 10\right)$}

51 Un petit tuyau (long. 3,2 cm, dia. 1,2 cm) (fig. 23 a), une tôle triangulaire avec des rivets $(5,2 \times 4,0 \mathrm{~cm})$ (fig. $23 \mathrm{~b}$ ) et un anneau en fer qui renforce une bordure en bronze (fig. 23 c), peuvent être attribués à un bassin à bec verseur (type Eggers 73/74). L'anneau en fer, de section carrée, est conservé en trois fragments, qui permettent de restituer un diamètre minimum de $29,5 \mathrm{~cm}$. Les fragments sont déchirés et pliés. La forme du bassin à bec verseur est presque inconnue pour la protohistoire, elle est en revanche très courante à l'époque gallo-romaine ${ }^{46}$ Pour La Tène D, H.-J. Eggers a fait la différence entre un type à panse rectangulaire (type Eggers 73) et un type à panse ronde (type Eggers 74$)^{47}$ Comme il ne disposait que de quatre exemplaires de ces bassins, sa typologie n'est pas assez précise ${ }^{48}$ pour une description plus détaillée, le détail technique de la construction du bord renforcé par un anneau en fer est de la même importance que la forme.

Aucune de ces deux formes ne figure dans les tombes celtiques jusqu'à récemment. Les exemplaires, connus depuis longtemps, se trouvent en Scandinavie du Sud et en Allemagne du Nord, comme dans la tombe 26 de Wiebendorf (Kr. Hagenow, D), avec un anneau en fer de section rectangulaire qui renforce le bord (fig. 24), comme à Verna (Keiling $1984: 8$, pl. 6). Ce bassin caractérise mieux le type Eggers 73 que son propre exemplaire de référence, plus proche des bassins romains ${ }^{49}$ H.-J. Eggers les a quand même considérés comme des importations celtiques en Europe du Nord à La Tène D. Les oppida ont seulement livré quelques petits fragments difficiles à identifier ${ }^{50}$ Une tombe riche de Raillencourt-Sainte-Olle (dép. Nord), fouillée en 2001, contient un bassin à bec verseur et à anse mobile très proche des exemplaires de Verna et Wiebendorf ${ }^{51} \mathrm{La}$ combinaison du bassin de Raillencourt-Sainte-Olle avec une patelle à manche de type Aylesford pose à nouveau la question de la fonction.

53 Les becs verseurs en forme de tête de chien, de la gueule duquel sort un tuyau comme ceux de Manching (Lkr. Pfaffenhofen a. d. Ilm. D) et de Kelheim (Stadt Kelheim, D) indiquent une variante locale ${ }^{52}$ Ces éléments sont aussi fixés par un rivet à un vase dont la forme reste inconnue. Un bassin à bec verseur avec une anse mobile en forme d'oméga venant de la tombe de Welwyn Garden City (Hertfordshire, GB) constitue une autre variante de ce genre (Stead 1967: 23-25 fig. 12-13): son fond comporte un omphalos et le vase est fermé par un couvercle-passoire en demi-lune. L'anse montre une origine romaine, mais les modifications indigènes (Stead 1967 : 25) attestent une utilisation locale pour les liquides à tamiser, peut-être des vins de fruits ou des boissons enrichies d'herbes.

Il est difficile de décider si ces types sont des récipients de tradition méditerranéenne ou de tradition celtique. La forme du bassin correspond bien aux bassins romains, mais le bec verseur riveté et le renfort en fer du bord ne correspondent pas aux traditions de la vaisselle romaine. Il évoque plutôt les chaudrons celtiques en bronze ou en fer à bord renforcé. En conclusion, si la forme de ces bassins est bien d'origine romaine, les versions connues sont plutôt des vases celtiques. Dans tous les cas, une production en Allemagne du Nord est exclue, comme pour toute la vaisselle métallique. 


\section{AUTRES FRAGMENTS : FOND $\left(\mathrm{N}^{\circ} 11\right)$}

Un fond plat (dia. environ 10,2 cm) avec des cercles concentriques interprétés comme des marques de tour ne peut être attribué à une forme connue (fig. 25). En tout cas, il $\mathrm{n}^{\prime}$ appartient pas à un gobelet type Idria et probablement pas à l'autre petite situle $\left(\mathrm{n}^{\circ}\right.$ 3). Il est possible qu'il fasse partie du récipient anciennement fermé par le couvercle ( ${ }^{\circ}$ 6).

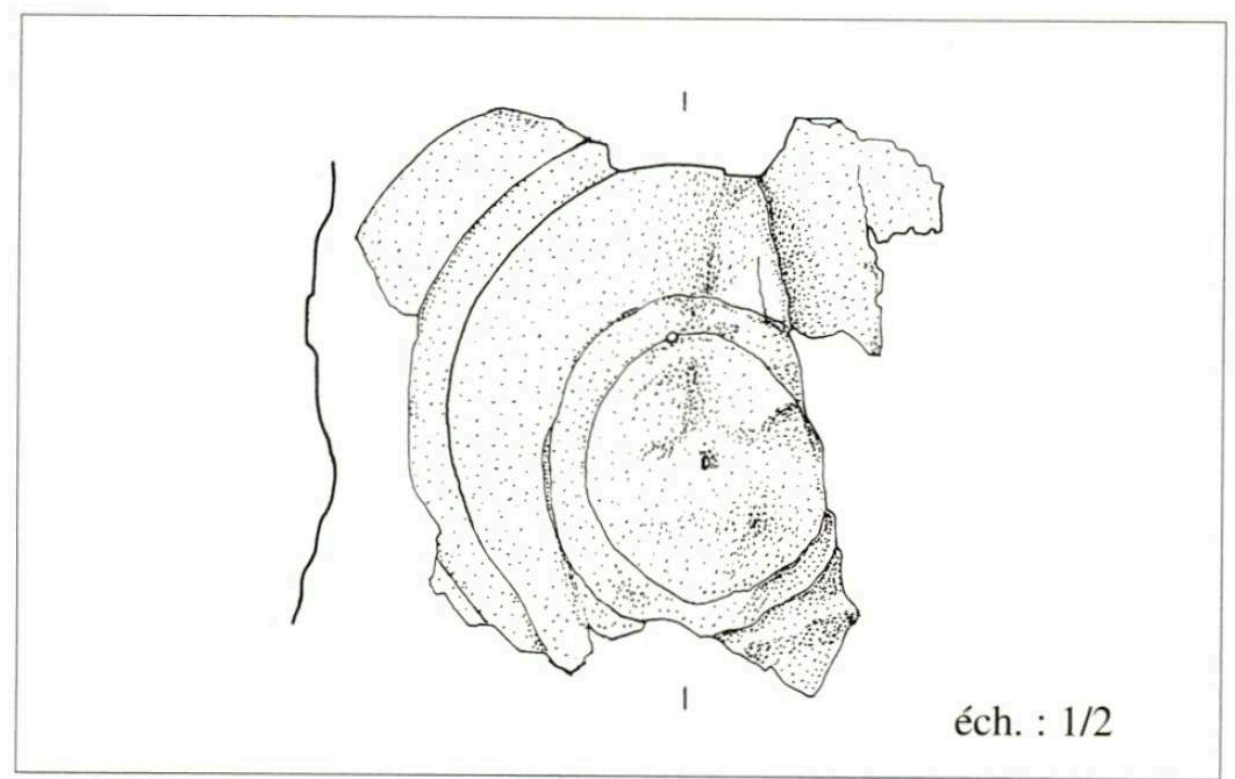

25-Fond d'un récipient (bronze) n 11

\section{LE BASSIN ETRUSQUE ( $\left.{ }^{\circ} 12\right)$. A.-M. Adam}

Parmi le matériel de la tombe à char de Verna, sont conservés plusieurs fragments d'un exceptionnel bassin étrusque de type podanipter (bassin pour le lavement des pieds). Ces fragments ne permettent pas de reconstituer le récipient dans son intégralité, mais celui-ci appartient à une catégorie d'objets plusieurs fois attestée par ailleurs et caractérisée par deux anses à décor plastique qui représentent des couples de lutteurs. 


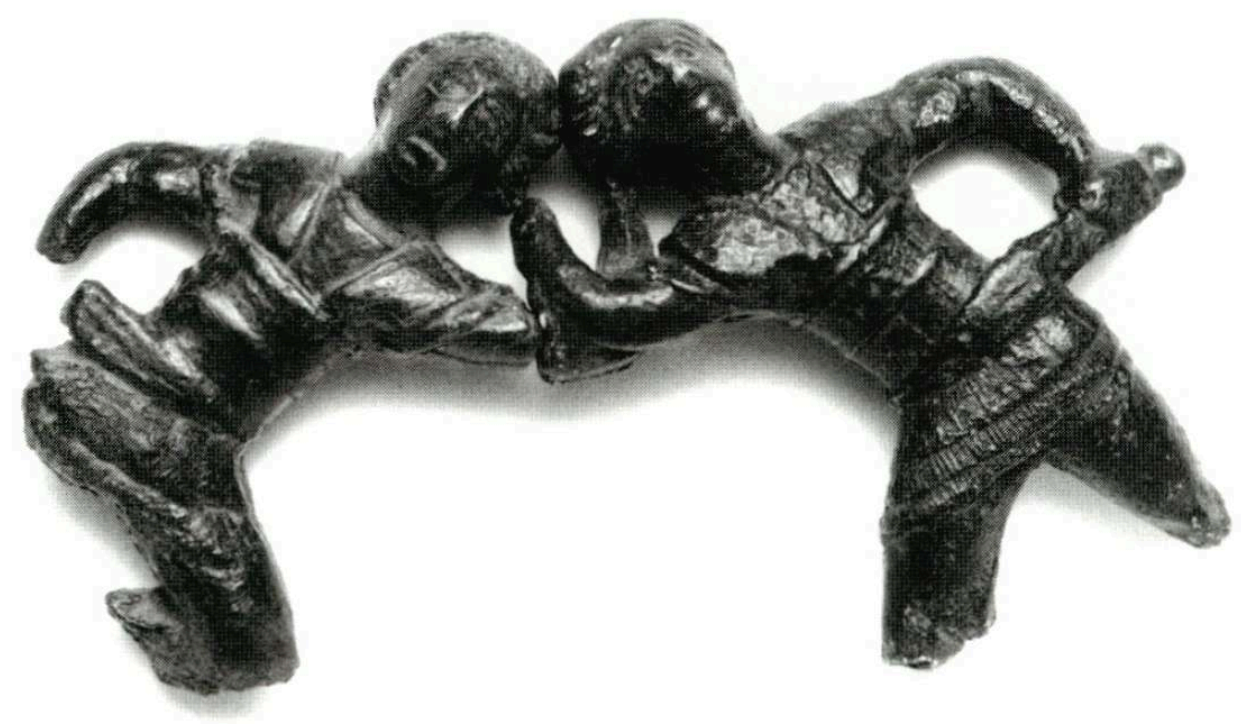

26 a -Anse du bassin étrusque, $n^{\circ} 12$

\section{DESCRIPTION}

57 La plus complète des deux anses de Verna est connue par un moulage ancien (inv. 90-14-1), l'original étant conservé dans une collection privée (fig. 26 a et b). L'essentiel du corps des lutteurs est conservé, sauf les jambes qui ont été brisées au niveau des cuisses et la main droite de l'un des personnages qui tenait, tout comme son compagnon, une épée dont on voit encore la pointe plaquée contre sa taille. Tous les deux sont vêtus d'une cuirasse à lambrequins et épaulières; ils sont tête nue, avec les cheveux coiffés vers l'arrière.

De l'autre anse, on conserve, outre plusieurs morceaux très corrodés de l'un des deux personnages (la tête et le haut du torse, le bassin, un bras et une jambe incomplets), le second guerrier, reconstitué à partir de plusieurs fragments (fig. 26 c). Il repose sur une grande palmette à sept pétales aigus. Entre les deux grandes volutes qui surmontent cette palmette, un bandeau est orné de croisillons incisés. Le haut de cette attache, de même que les pieds de la statuette, sont très corrodés. L'attache est traversée par plusieurs rivets en fer (l'un sur le bandeau supérieur, l'autre sous le cœur de la palmette entre le deuxième et le troisième pétale à gauche). Ces rivets, disposés de façon irrégulière et sans le moindre soin, semblent témoigner d'une tentative maladroite de restauration. 


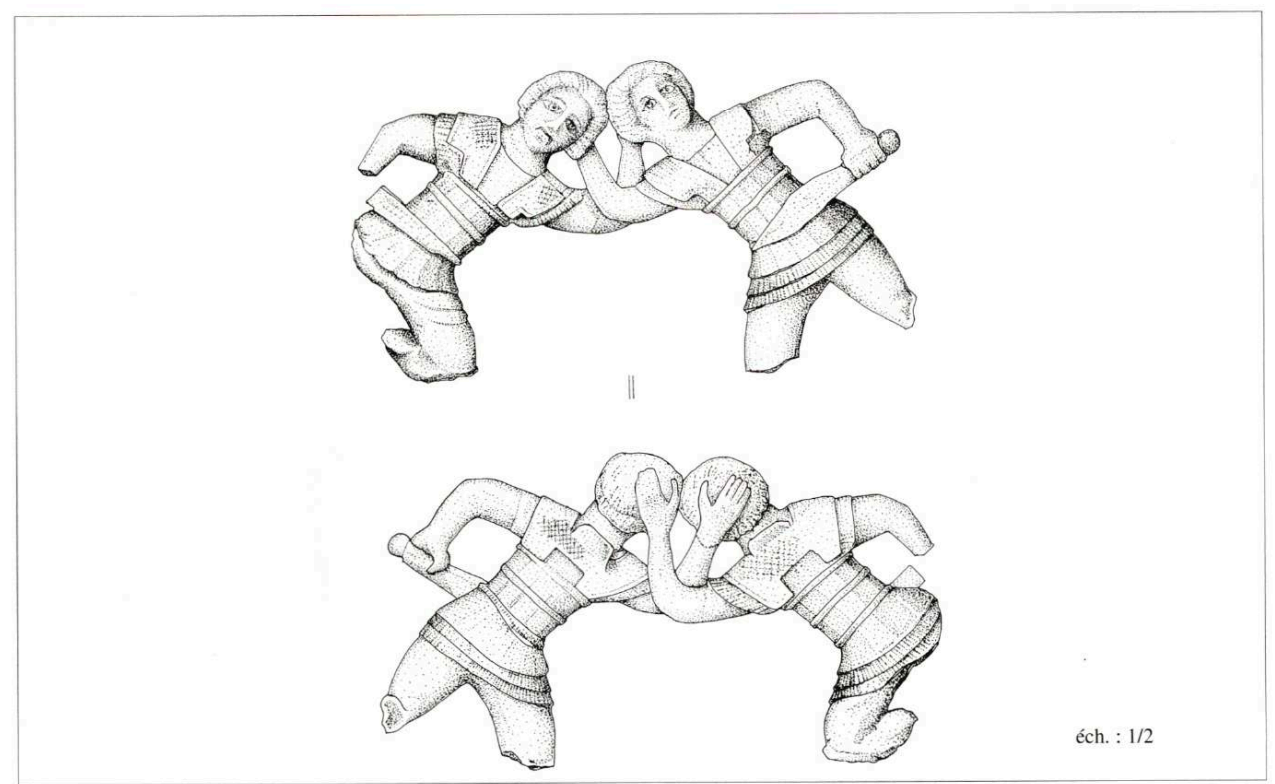

$26 \mathrm{~b}$ - Anse du bassin étrusque, dessin d'après un moulage ancien, $n^{\circ} 12$ tordus et brisés certainement volontairement. Cette destruction volontaire est intervenue avant le contact avec le feu qui a fortement corrodé et même fondu l'essentiel des fragments. On constate, en effet, que cette action du feu s'est exercée de façon inégale selon les morceaux, voire même d'un côté à l'autre des mêmes fragments. La présence d'un morceau de bord complètement fondu auquel adhère encore une parcelle d'os incinéré fournit l'explication du traitement subi par le récipient : après destruction volontaire et violente, il a été probablement jeté sur le bûcher funéraire, où ses morceaux dispersés ont été soumis à une chaleur plus ou moins forte.

Les mêmes signes d'une détérioration délibérée et d'un passage au feu marquent les autres récipients en bronze de la sépulture (supra) et cette application commune d'un traitement exceptionnel confirme l'appartenance du bassin étrusque au contexte de la sépulture principale du tumulus, malgré les questions que soulève sa présence même dans un ensemble funéraire de La Tène Finale. 
62

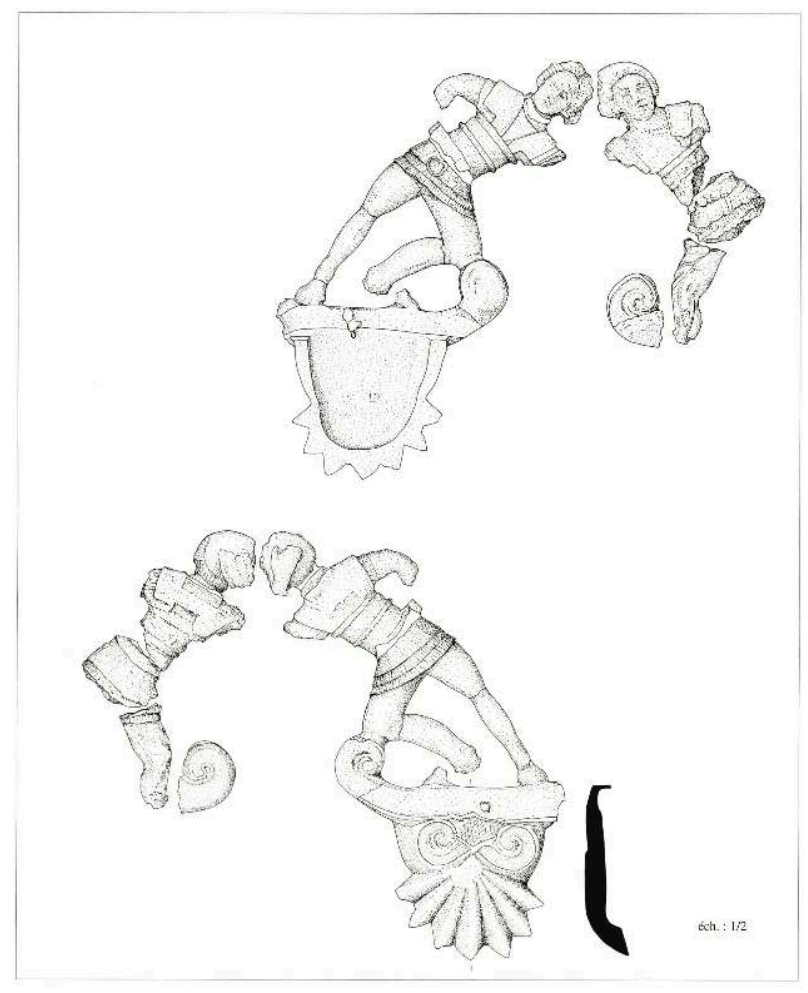

26 c - Anse du bassin avec attache, $n^{\circ} 12$

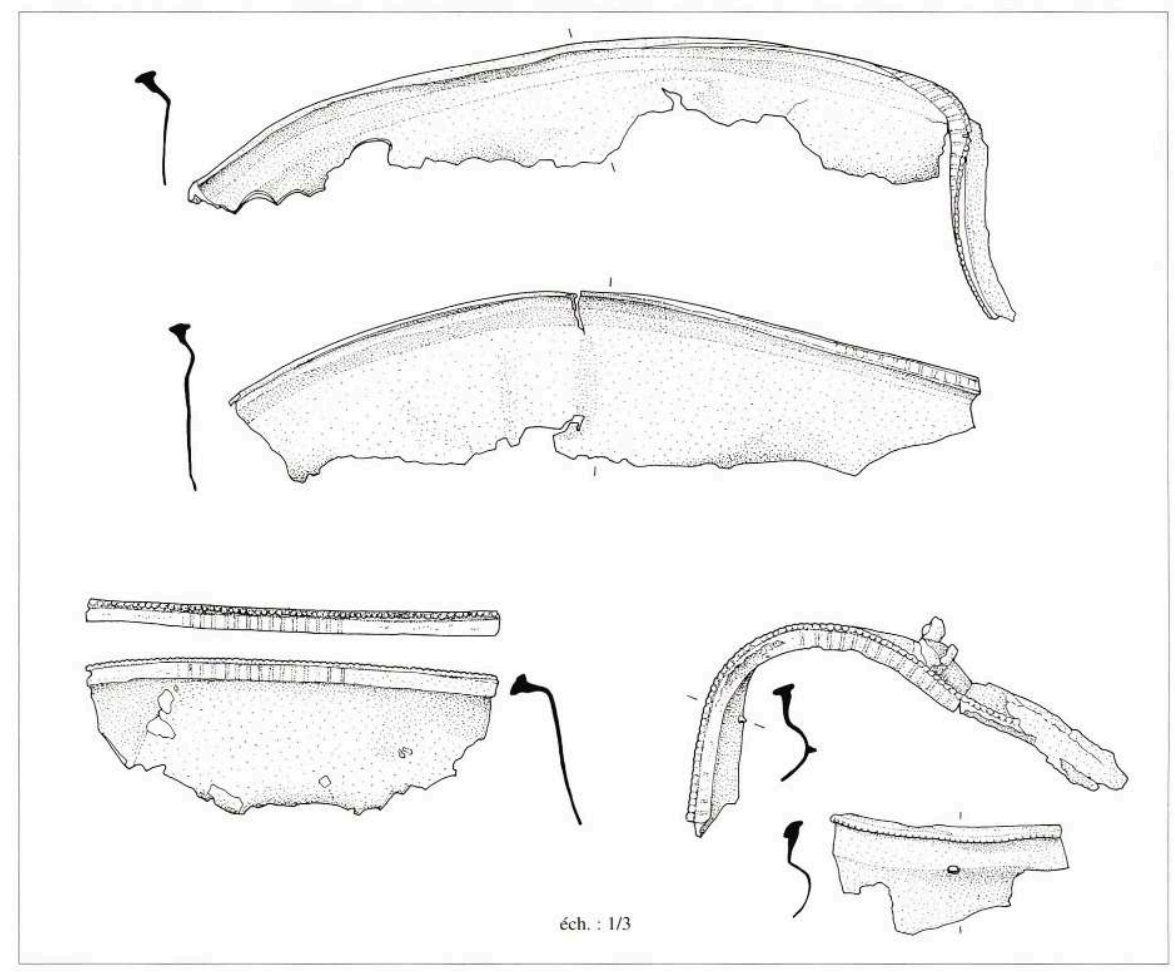

27-Bord du récipient 


\section{TYPOLOGIE ET COMPARAISONS}

63 En effet, si les autres pièces du service en bronze constituent un groupe homogène de vaisselle tardo-républicaine, le bassin aux lutteurs se rattache, lui, à une catégorie, recensée à plusieurs reprises et nettement plus ancienne, de récipients originaires d'Italie (Boucher 1986; Kimmig 1990). Même si l'on peut distinguer, à l'intérieur du type général, plusieurs séries en fonction de l'aspect des couples de personnages qui ornent les anses, le schéma d'ensemble est plusieurs fois reproduit, comme le montrent les deux bassins conservés complets (bassin provenant sans doute du Picénum, au musée de Boston - $\mathrm{n}^{\circ} 1$ de notre liste ; bassin trouvé dans la tombe II de la nécropole de Filottrano - $\mathrm{n}^{\circ} 6$ de la liste, $c f$. p. 57) (fig. 28,29).

Ce sont des bassins larges et peu profonds, pourvus de chaque côté de deux anses horizontales plastiques: deux personnages luttant, corps inclinés et têtes appuyées l'une contre l'autre; chacun des personnages repose sur un support qui permet la fixation de l'anse à la cuve et se compose d'une grande palmette, complétée par des volutes et une tige terminée en fleuron.

Un certain nombre de détails morphologiques distinguent, en fait, le bassin de Boston, qu'il faut sans doute considérer comme le prototype de la série (fig. 28). Sa cuve, plus profonde et de profil arrondi, repose sur un petit pied annulaire : la forme s'apparente donc à celle des bains de pieds connus dans le monde grec, en particulier par des représentations figurées (Milne 1944: pl. I-II). Un jonc décoratif (oves et perles) est rapporté sur le bord droit du récipient. Les deux lutteurs de chacune des anses combattent face à face : leur corps est donc vu de profil, sauf la tête tournée de troisquarts. Les deux têtes sont appuyées l'une contre l'autre et les personnages s'agrippent mutuellement les deux bras. La composition des attaches est très complexe, avec plusieurs éléments superposés : une palmette formée de sept pétales à extrémité bouletée et surmontée de deux doubles volutes; puis trois larges pétales retombants, d'où émergent à leur tour une tige redressée, terminée par un motif floral large et plat, à petit bouton central, et une tige plate recourbée en volute. Au-dessus de cette dernière, une courte tige en forme de double volute sert de support au genou fléchi de chaque personnage. Les deux anses plastiques sont complétées par deux poignées mobiles ovales, placées elles aussi en vis-à-vis sur le bord de la cuve et maintenues par une bobine moulurée, décorée comme les poignées elles-mêmes de bagues de motifs de perles.

Podanipteres àlutteurs, liste des exemplaires connus

\section{Lutteurs nus}

1. Boston, Museum of Fine Arts, inv. $n^{\circ} 03.999$, provenance : "Picénum" (Pernice 1925 : 36-37, pl. 9 ; Milne 1944, n 85 ; M. Comstock, C.C. Vermeule, Greek, Etruscan and Roman Bronzes in the Museum of Fine Arts. Boston, 1971, n 429 ; Rolley 1983, $n^{\circ} 130$, p. 140 ; Boucher 1986, n 8, fig. 14-15; Kimmig 1990, n A1, p. 83, pl. 4).

2. Darmstadt, Hessisches Landesmuseum, provenance : Borsdorf (Boucher 1986, $\mathrm{n}^{\circ}$ 6, fig. 10 ; Kimmig 1990, n A2, p. 83, pl. 2-3 ; Menke 1991).

3. Bologne, Museo Civico, Archeologico (Collection Palagi), provenance inconnue (II Museo Civico, Archeologico di Bologna. Bologna, 1982, p. 191-192 ; Boucher 1986, n 7, fig. 12-13; Kimmig 1990, n A3-4, p. 83).

\section{Guerriers}

4. Paris, Musée du Louvre, provenance inconnue (A. de Ridder, Les bronzes 
antiques du Louvre II. Paris, 1915, n² 2631, pl. 95 ; Provost 1983 :213, fig. 4 ; Boucher 1986, n² 2, fig. 3-4 ; Kimmig 1990, nº B2, p. 83).

5. Londres, British Museum, provenance inconnue (H.B. Walters, Catalogue of the Bronzes, Greek, Roman and Etruscan in the Department of Greek and Roman Antiquities, British Museum. Londres, 1889, n 674 ; Flaynes 1985, n 153, p. 213 ; Boucher 1986, n 1, fig. 1-2 ; Kimmig 1990, n B3, p. 83).

6. Ancone, Museo Arch. Nazionale delle Marche, provenance : Filottrano, Santa Paolina, Tombe 2 (Baumgärtel 1937, pl. XX ; Boucher 1986, $\mathrm{n}^{\circ}$ 4, fig. 6-7 ; Landolfi 1987, p. 452-454; Kimmig 1990, nº B1, p. 83).

7. Vienne, Kunsthistorisches Museum, provenance inconnue (Maule 1977, p. 498-499, fig. 14 ; Boucher 1986, n 3, fig. 5 ; Kimmig 1990, nº B4, p. 83).

8. Hières-sur-Amby (Isère), provenance : Verna.

9. Angers, Musée, provenance : Sainte-Gemmes-sur-Loire (Maine-et-Loire) (Provost 1983, fig. 2-3 ; Boucher 1986, n 5, fig. 8-9 ; Kimmig 1990, n B5, p. 83 ; Jannot 1995, p. 83).

\section{Palmette isolée}

10. Provenance : Barzan (Charente-Maritime), Le Fâ, fouilles K. Robin (2001) (cf.note 76).
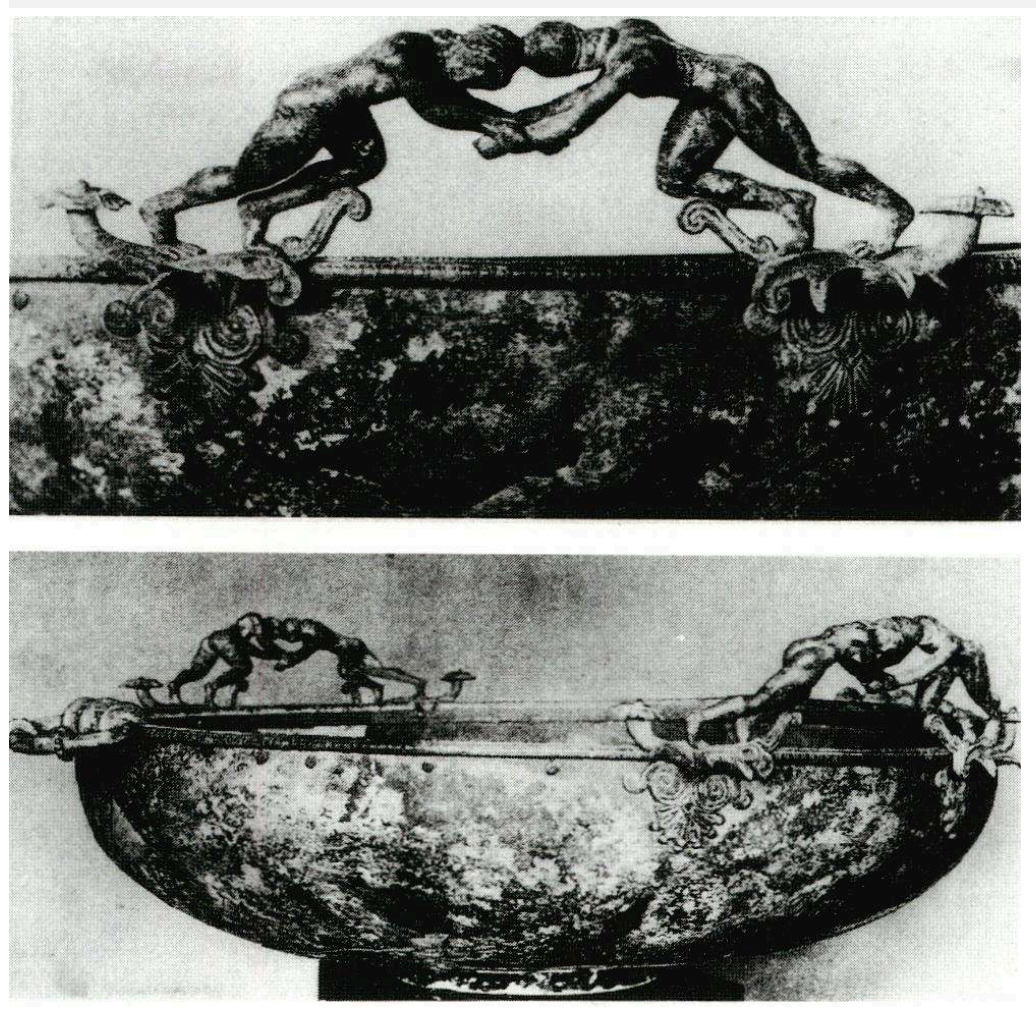

28- Bassin à lutteurs, d'après Pernice 1925, pl. 9. Musée de Boston, 

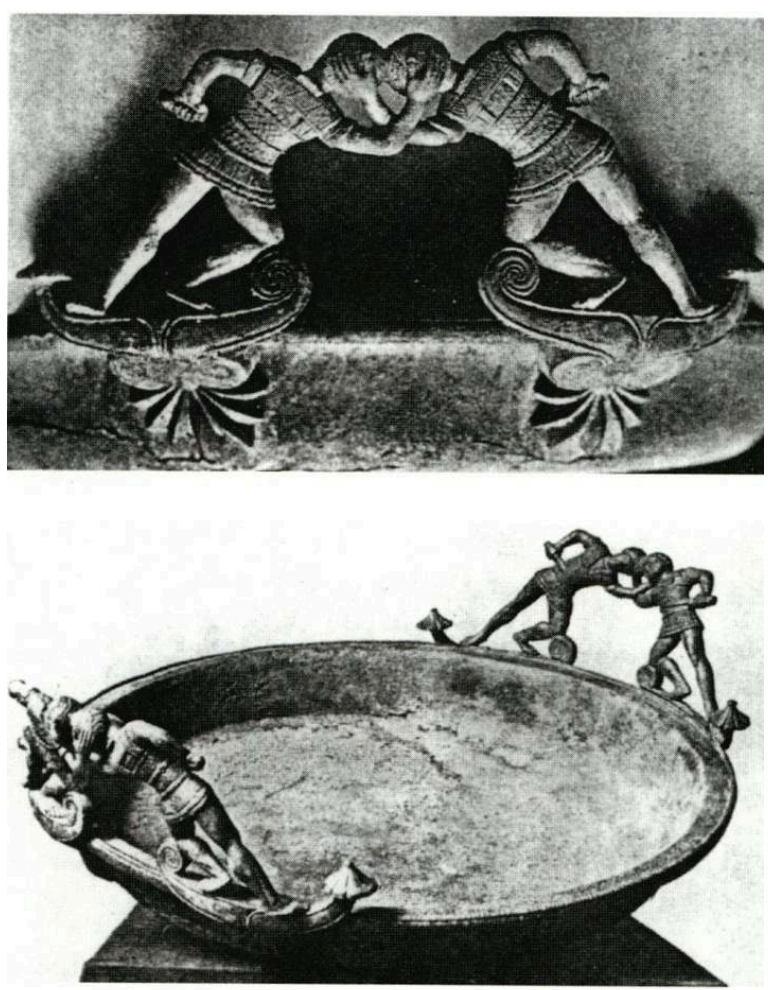

29- Bassin à lutteurs, nécropole de Filottrano (Italie), d'après Baumgartel 1937, pl. XX

On peut considérer que ce bassin, dont, selon les indications muséographiques successives, la provenance a été attribuée tour à tour à l'Italie méridionale et à la côte adriatique (Picénum) (Pemice 1935: 36), constitue sur le plan typologique un intermédiaire entre les modèles grecs que nous présenterons plus loin et les séries étrusques. De style assurément non grec, les lutteurs allient à la rondeur encore très ionisante de certaines parties de leur corps un dynamisme du mouvement que l'on rencontre surtout dans l'art tardo-archaïque d'Italie centrale. On peut supposer que ce bassin a été fabriqué, dans le premier tiers du Ve s., par un atelier étrusco-campanien ${ }^{53}$ ce qui rendrait bien compte de son caractère hybride : en effet, certains de ses détails morphologiques, comme les poignées ovales fixées par une bobine à trois moulures, n'apparaissent guère en Etrurie et sont au contraire largement présents sur les récipients de bronze grecs ${ }^{54}$ Même si nous manquons pour les figures de lutteurs de comparaisons précises, une certaine parenté avec des statuettes décorant les lébès de Capoue $^{55}$ confirme cette probable origine campanienne.

Les autres exemplaires du type (il s'agit, sauf pour l'objet trouvé à Filottrano, de poignées détachées de leur bassin d'origine) ont tous en commun la position frontale des lutteurs, qui se tiennent de profil l'un par rapport à l'autre, et surtout le modèle des attaches, plus simples que pour le bassin de Boston : la palmette à sept pétales aigus autour d'un petit coeur losangique est surmontée de deux grandes volutes, "liées" entre elles par un bandeau, rectangulaire ou losangique, qui porte un motif incisé différent d'un exemplaire à l'autre (généralement des croisillons et sur la poignée de Borsdorf des triangles hachurés entre deux lignes de perles). Au-delà de cette barrette, les prolongements des volutes s'écartent de part et d'autre pour épouser le bord du bassin; leurs extrémités redressées se terminent, d'un côté par une nouvelle volute où 
s'appuie le genou fléchi du lutteur, de l'autre par une courte tige quadrangulaire et un fleuron conique dont les pétales arrondis se rejoignent au sommet en une petite boule.

Seul le bassin complet de Filottrano (et les fragments très endommagés de celui de Verna) (fig. 29) permettent de restituer l'aspect des récipients, qui se distinguaient du bassin de Boston par une profondeur moindre, un fond plat et probablement une absence de pied. Le rebord plat porte sur son pourtour externe une lèvre un peu épaissie, décorée d'une ligne de perles et d'une bande d'oves.

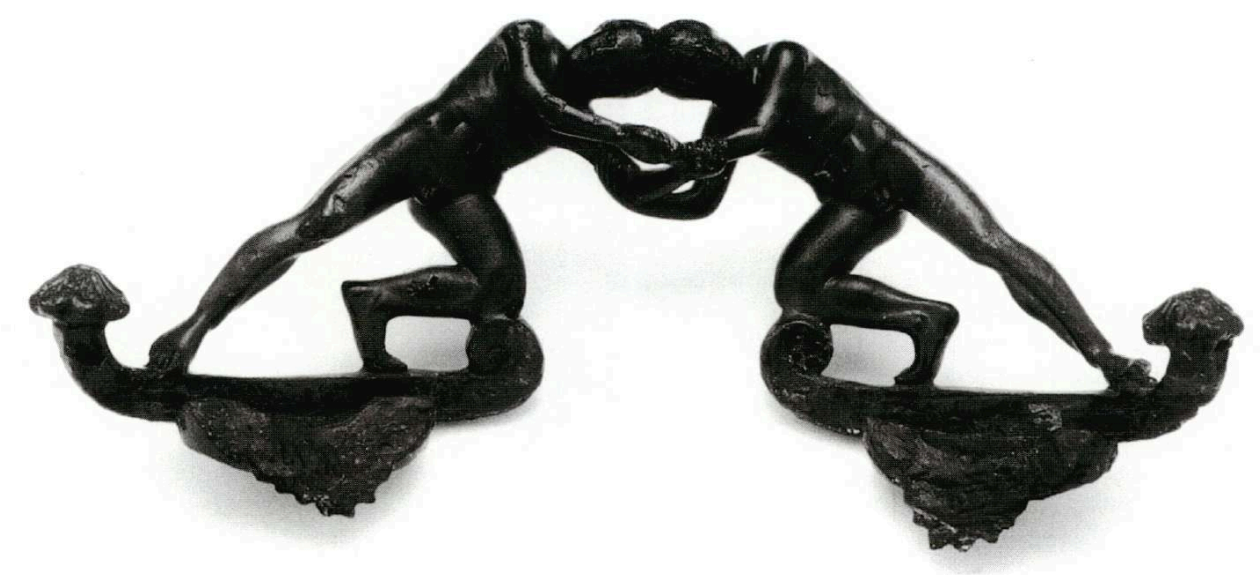

30-Anse d'un bassin à lutteurs : Borsdorf (Wetteraukreis, D)

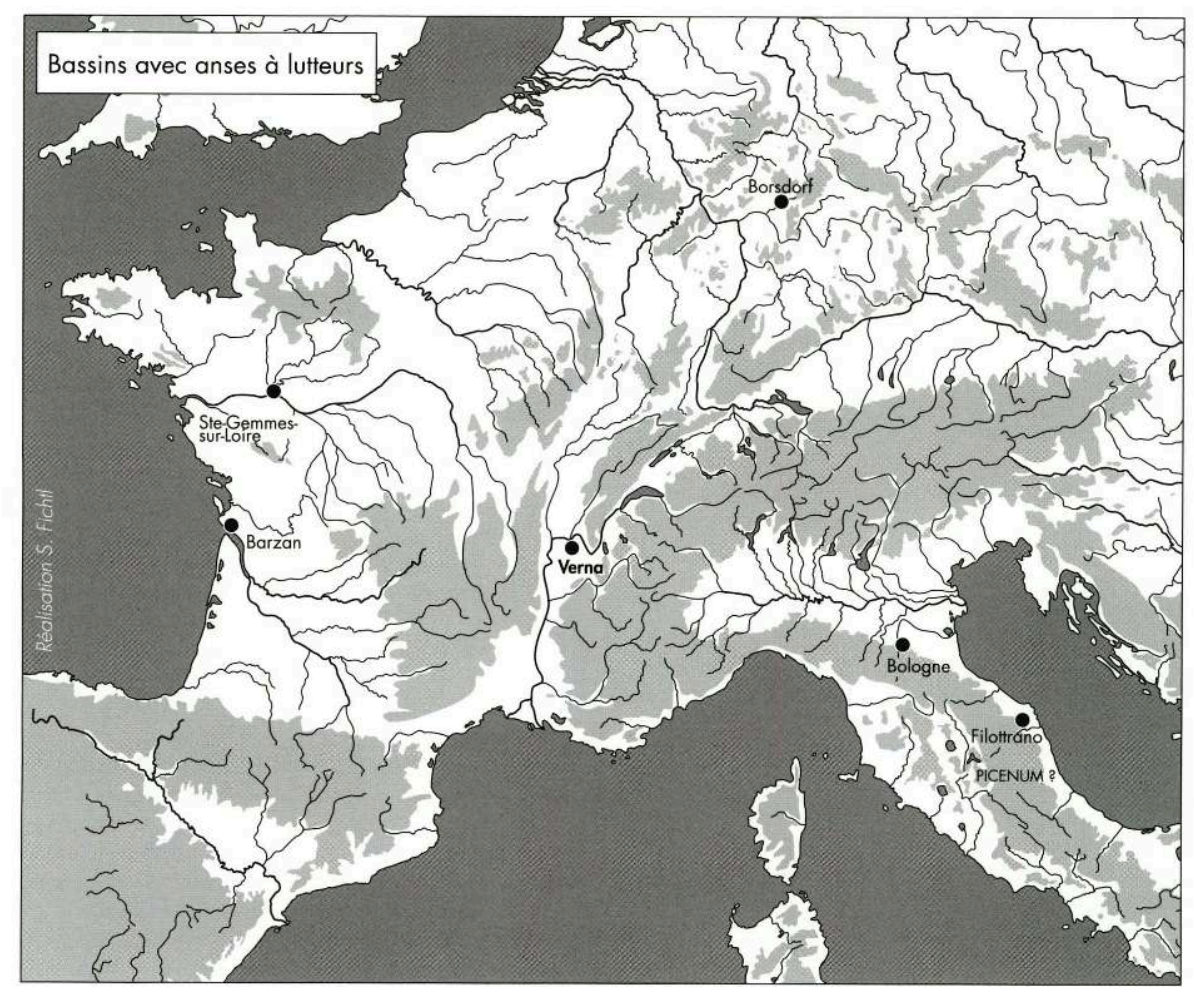

31-Répartition des bassins à lutteurs

69 Au-delà de cette relative unité morphologique, l'aspect et le style des figures de lutteurs peuvent varier sensiblement d'un exemplaire à l'autre. En dehors du bassin de Boston, deux autres objets sont caractérisés par la nudité des personnages: l'anse trouvée à Borsdorf, en Hesse (liste, $n^{\circ}$ 2) (fig. 30), se rapproche de celles de Boston par 
le geste des deux hommes qui se saisissent mutuellement les bras. Au contraire, les lutteurs de la paire d'anses de Bologne (liste, $\mathrm{n}^{\circ} 3$ ) s'empoignent par la chevelure ; leur main opposée, appuyée sur la hanche, pourrait, d'après sa position, avoir tenu une épée, mais aucune trace de celle-ci n'est conservée.

Un second groupe, d'aspect plus homogène, réunit toutes les autres poignées, où les personnages, tête nue, sont revêtus d'une cuirasse à épaulières et lambrequins et armés d'une épée (liste, $n^{\circ} 4$ à 9).

\section{L'ORIGINE DES BASSINS À LUTTEURS}

71 En dehors de ce type particulier des bassins à décor plastique, d'autres modèles de podanipter sont connus en Italie centrale dès le Vie s. av. J.-C., en particulier sur le flanc oriental de la péninsule (fig. 31) : leur cuve assez profonde, à profil arrondi, repose sur trois pieds minces d'inspiration animale (pattes de fauve ou d'équidé), fixés à la paroi du bassin par des plaquettes incurvées qui en épousent le galbe. Ils sont caractérisés surtout par la forme des anses arrondies ou rectilignes, coulées d'une seule pièce avec leur plaque d'attache, qui dessine sur son bord inférieur deux arcs surbaissés. Ce modèle est attesté dans les riches tombes de la seconde moitié du VIe s. en Ombrie (Monteleone di Spoleto et Castel San Mariano), à Orvieto et en Romagne (San Giovanni in Compito) ${ }^{56} \mathrm{Il}$ a été exporté en Italie méridionale (Vaste, tombe 569)(Rolley 1991: 204 et pl. XIII) et un exemplaire a été trouvé également en Slovaquie ${ }^{57}$.

72 Pour ce qui concerne l'Italie méridionale, on y rencontre plus fréquemment les podanipteres de type grec où les trois pieds de lion, de plus grande dimension, sont réunis par une couronne de bronze où vient se poser le fond de la cuve ${ }^{58} \mathrm{D}$ 'autres sépultures, à la périphérie du monde grec, en ont livré un nombre important (à Trebenischte ou Novi Pazar en Illyrie) et la fabrication de ces objets grecs a été attribuée, soit à des ateliers corinthiens, soit aux ateliers de Grande-Grèce (Rolley 1991 : 196 ; Gauer 1991 : 79-81). En Etrurie, le modèle classique du podanipter grec n'a pas été retrouvé, mais il est illustré à plusieurs reprises dans des scènes de banquet à Chiusi, dans la peinture funéraire ("Tomba del Colle") ou sur les cippes à reliefs (Adam 1995 : 106, 113, fig. 2).

73 Werner Gauer (Gauer 1981; Gauer 1991: 70-82) a réparti les bains de pieds grecs en plusieurs classes en fonction de l'aspect de leurs anses. Un premier groupe, attesté par de nombreux exemplaires à Trebenischte et quelques-uns également en Italie méridionale, est caractérisé par des poignées à attaches discoïdes (Scheibenattasche), en forme de rosettes ou rosaces de différents modèles. Ce type de poignée apparait un peu avant le milieu du VIe s. Les plaquettes de fixation peuvent être décorées également d'une palmette gravée, surmontée d'une double volute ${ }^{59}$.

Dans l'autre groupe, la fixation des poignées à la cuve se fait de chaque côté au moyen de deux éléments incurvés (Bogenattasche) puis redressés au-dessus du bord du récipient, pour se terminer en un motif floral (rosace, bouton) ou animal (serpents ou protomès de bélier) ${ }^{60}$ Une autre rosace se dresse souvent perpendiculairement au centre de la poignée elle-même. De façon générale, les exemplaires de ce second groupe se caractérisent par la richesse plus grande de leurs décors. Dans le podanipter de NewYork publié par M. J. Milne, le système d'attache des poignées est complété par deux grandes palmettes avec double volute, appliquées sur le bassin avant l'appendice arqué, lui-même terminé par un bouton floral (Milne 1944 : fig. 2) (fig. 32). 


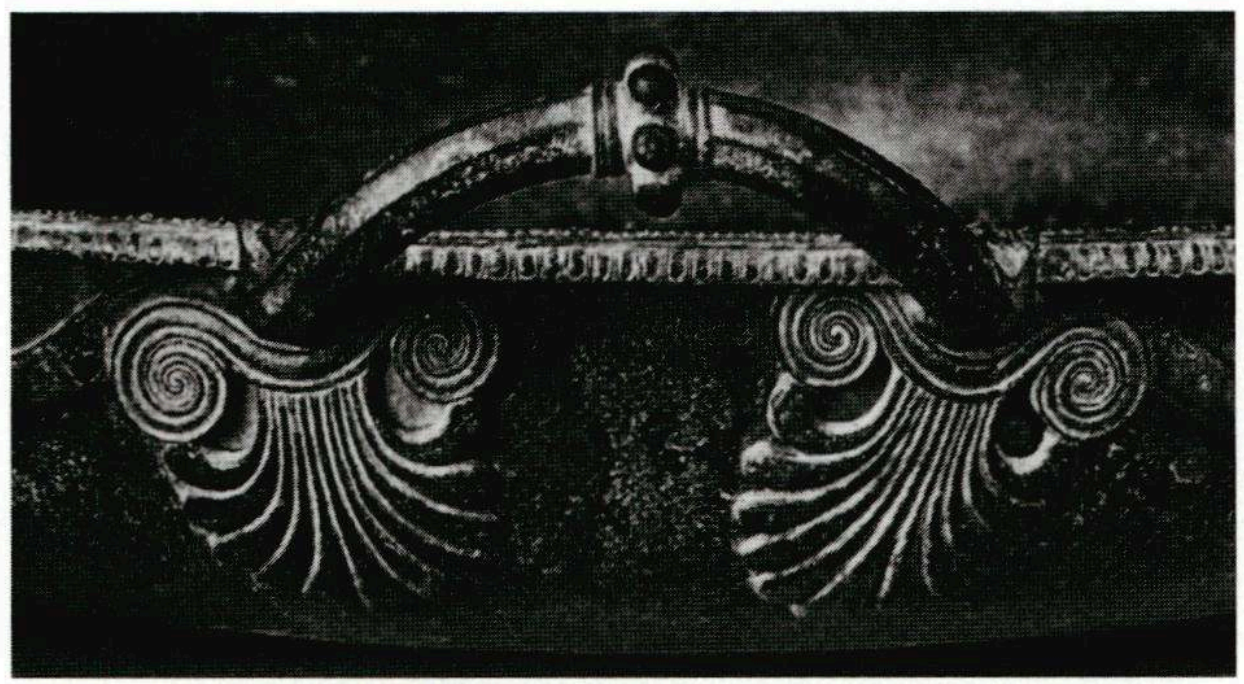

32-Anse du podanipter de New York, d'après Milne 1944, fig. 2

75 Le corps de l'anse peut être remplacé par un motif plastique : le plus souvent, il s'agit de deux lions couchés, opposés par leur arrière-train, de part et d'autre d'une rosace ${ }^{61}$; une variante (attestée à Dodone et sur l'Acropole d'Athènes) remplace les lions par deux lézards disposés de la même façon ${ }^{62}$ Un bassin complet du British Museum présente une autre variante encore : les anses plates à attaches arquées, terminées en serpents, portent chacune un couple de banqueteurs étendus côte à côte. Ce bassin n'est pas grec: il a été attribué de façon hypothétique à la Campanie ou encore à l'Etrurie méridionale (les visages des banqueteurs, avec leur profil aigu, rappellent en effet des antéfixes campaniennes mais aussi certaines figures de terre cuite fabriquées à Cerveteri à la fin du VIe s. $)^{63}$ Ce bassin, sans doute un peu antérieur au bassin à lutteurs de Boston (dernier quart du VIe s.), constituerait donc un autre exemple d'adaptation étrusco-campanienne des modèles grecs.

Si nous poursuivons l'inventaire des types d'anses connus dans la production grecque, une dernière catégorie peut apparaître comme le plus proche modèle des poignées italiennes à lutteurs. Etudiée en détail par W. Gauer (1981) à partir d'un exemplaire d'Olympie $^{64}$, elle comporte un riche décor plastique : deux lions affrontés se disputent un cervidé placé entre eux ; leurs pattes postérieures reposent sur le système d'attache de la poignée : une palmette surmontée d'une tige à fleuron et d'une grande volute. En dehors du fragment d'Olympie, deux autres exemplaires seraient connus : l'un d'eux, provenant de l'Acropole d'Athènes, a conservé d'un côté la palmette de fixation complète (Gauer 1981 : 123-125, fig. 54-55 et 59) (fig. 33). Un groupe de trois animaux, conservé à Copenhague (Musée Thorvaldsen) pourrait être complété, selon la reconstitution proposée par W. Gauer, par un fragment d'attache trouvé à Locres (Gauer 1981: 124-128, fig. 56 à 58 et 60) (fig. 34 a et b). Il faut donc considérer le modèle représenté par ces trois anses comme le véritable prototype de nos anses à lutteurs, à la fois pour ce qui est du schéma des combattants affrontés, dont les corps inclinés forment l'arc de la poignée, et pour le système d'attache dont les bassins étrusques semblent s'être directement inspirés.

77 Les trois poignées couvrent, selon la datation proposée par W. Gauer (1981:142), une période étendue : le groupe de l'Acropole, considéré comme le plus ancien, est situé vers 540 , celui de Copenhague-Locres à la fin du VIe s. et le groupe d'Olympie à 
l'extrême fin de l'archaïsme (vers 480). Les trois exemplaires peuvent être issus d'ateliers différents : à l'origine de la série, Gauer restitue une production d'un atelier attique, il rattache la poignée d'olympie plutôt à l'activité des ateliers corinthiens, et pour celle de Copenhague-Locres, il admet la possibilité d'une fabrication en Italie méridionale, probablement à Locres même.

Sans entrer dans le détail de son argumentation ni à plus forte raison dans le débat récurrent concernant l'attribution de nombreux récipients en bronze à des ateliers corinthiens ou sud-italiens, il est surtout intéressant de constater que de nombreux modèles de podanipteres grecs sont connus sur les sites de Grande-Grèce, notamment les plus richement décorés et en particulier celui décoré d'animaux combattants, dont le premier représentant de notre série à lutteurs paraît s'être directement inspiré, avec un décalage de quelques décennies seulement. Rappelons que le schéma des lutteurs de profil, penchés l'un vers l'autre et se tenant par les poignets, est connu par ailleurs dans l'iconographie étrusque dès le VIe s. (par exemple dans la célèbre tombe peinte "des Augures", à Tarquinia, vers 530). Le modèle du bassin à anses plastiques est ensuite repris et mis en oeuvre, avec un nouveau décalage (du moins dans l'état actuel de nos connaissances) par des ateliers d'Etrurie qui, au terme de l'évolution, modifient encore le schéma, en revêtant les deux combattants affrontés d'une cuirasse et en les armant d'une épée.

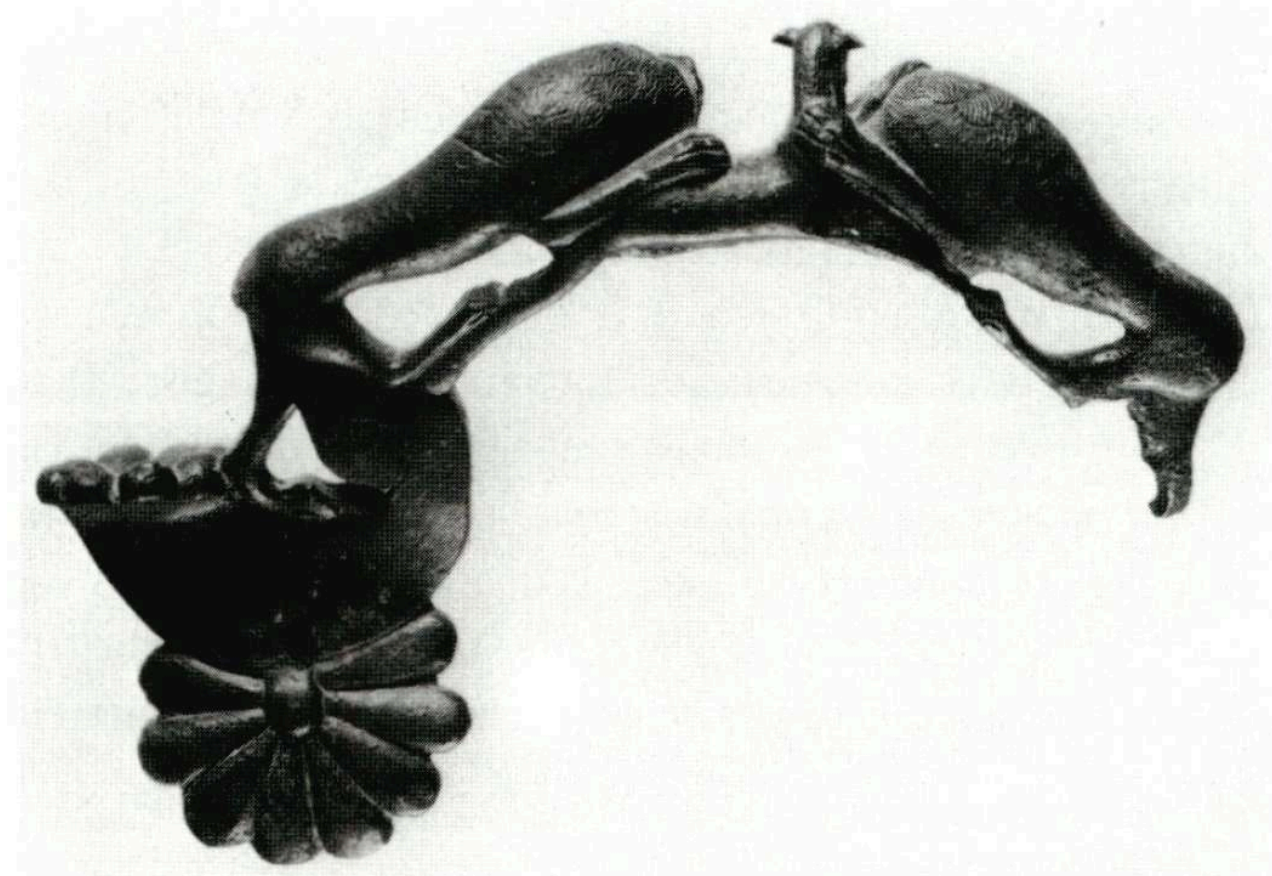




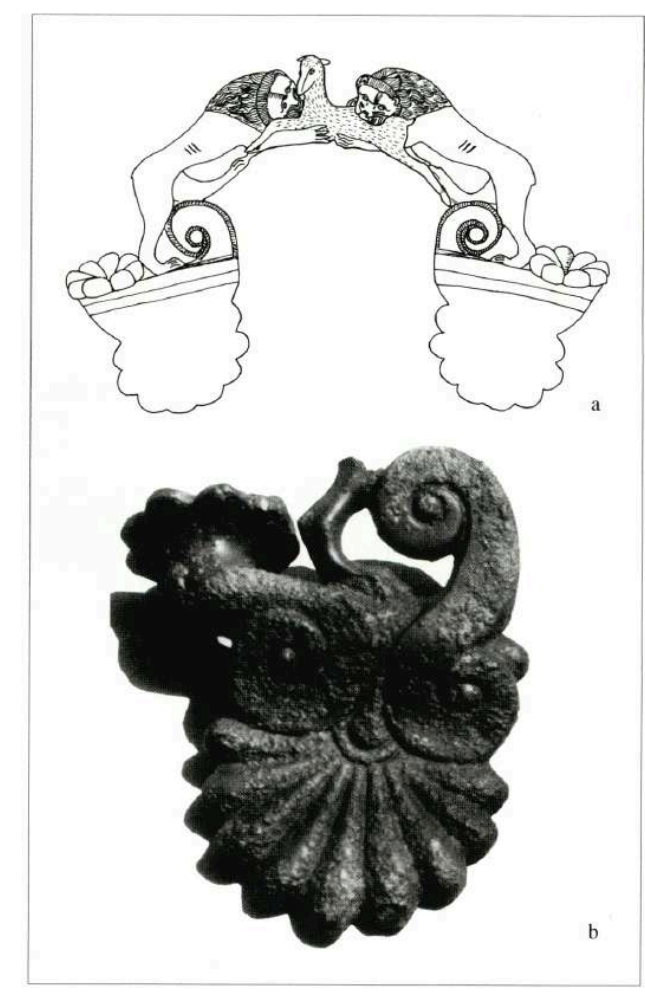

34 a -Restitution proposée par Gauer ; b : palmette de Locres, d'après Gauer 1981

\section{STYLISTIQUE ET DATATION}

79 Sur le plan stylistique, les trois poignées présentant des lutteurs nus sont assez sensiblement différentes les unes des autres et proviennent probablement d'ateliers distincts. La paire conservée au Museo Civico de Bologne n'a pas de provenance archéologique connue, mais il est fort probable qu'il s'agisse d'une découverte locale ou du moins originaire d'Etrurie padane. Un certain nombre de rapprochements avec des petits bronzes de cette région, en particulier plusieurs couronnements de candélabre caractéristiques de la production padane ${ }^{65}$, permettent de supposer que les ateliers de ce secteur ont produit également notre type de bain de pieds. Ces figures ont en commun avec celles qui nous occupent le traitement anatomique (aux proportions plutôt trapues, avec le cou épais, la musculature du torse accentuée) et aussi le style du visage, avec de grands yeux en amande, cernés d'un liseré saillant. Ces comparaisons, dont la datation s'appuie à la fois sur des critères stylistiques et, surtout pour Spina, sur les contextes archéologiques, autorisent à proposer, pour la paire d'anses de Bologne, une datation vers 400 .

La situation de la poignée de Borsdorf (fig. 30), en termes de style et d'atelier, est plus délicate à définir. Son aspect renvoie globalement à la production de l'Etrurie intérieure, qui a comporté probablement de la fin du VIe s. jusqu'au IIIe s., plusieurs centres (Orvieto, Chiusi, l'Etrurie septentrionale), dont les caractéristiques spécifiques ne sont pas toujours faciles à cerner, du fait des liens qui ont dû se tisser entre eux et d'une certaine communauté d'inspiration, résultant d'un contact privilégié avec les produits du classicisme grec (Maule 1977 ; Dohrn 1982 ; Cristofani 1985 : 33-34). On peut considérer que les ateliers d'Etrurie padane constituent aussi, par les relations que cette région a entretenues avec l'Etrurie septentrionale interne, et également avec 
Chiusi, un prolongement de ce courant. D'autant plus que la plaine du Pô et surtout les ports du delta, par leurs contacts privilégiés, au Ve s., avec la culture grecque, ont certainement joué un rôle de premier plan dans la transmission des schémas plastiques d'origine hellénique.

D'ailleurs, les statuettes de Borsdorf rencontrent elles aussi des parallèles dans la petite plastique de la zone padane, par exemple le célèbre "Ephèbe Fruga" (statuette d'offrant de Monte Capra) ou l'Hercule drapé dans la léontè, trouvé dans le sanctuaire de Villa Cassarini à Bologne ${ }^{66}$. Plus largement, on trouve des rapprochements dans le nord de l'Etrurie, comme plusieurs figures, de taille diverse, dans le dépôt de Monte Falterona Le bronze de Borsdorf est peut-être un peu plus ${ }^{67}$. ancien que la paire d'anses de Bologne, mais pas de façon notable (dernières années du Ve s. av. J-C., ou vers 400 ?).

$C^{\prime}$ 'est probablement aussi dans ces régions septentrionales qu'a lieu la transformation des lutteurs en guerriers. On y rencontre, en tout cas, dès la seconde moitié du Ve s., la figure du guerrier en armes sur des cimaises de candélabre ${ }^{68}$, tout comme parmi les bronzes votifs ${ }^{69}$. Un certain nombre d'entre eux, sur les candélabres, présentent la même tenue que les figurines des bassins : cuirasse laissant dépasser légèrement le bord plissé de la tunique, absence de casque. On peut citer, en particulier, les deux groupes, presque semblables, de Bologne et de Paris, où le guerrier est associé à un homme âgé barbu, et qui doivent dater de la fin du Ve s. (Sassatelli 1987, fig. 4-5-11 et 6 à 9), ou encore une autre paire de cimaises, un peu plus récentes (première moitié ou milieu du IVe s.), avec deux jeunes guerriers côte à côte ${ }^{70}$ (fig. 35).

Les six anses ou paires d'anses ornées de guerriers ne sont pas toutes contemporaines, comme le style de chacune permet de le reconnaître : elles doivent jalonner une partie du IVe s. La plus ancienne de la série pourrait être l'anse isolée du Musée du Louvre ( ${ }^{\circ}$ 4 de notre liste, vers 375 ?), suivie de peu par celle du British Museum ( $\left.n^{\circ} 5\right)$ qui lui est apparentée par plusieurs aspects (traitement des visages et des chevelures). Le seul des bassins trouvé dans un contexte datable (Filottrano, Santa Paolina, tombe II, $\mathrm{n}^{\circ} 6$ de notre liste) (fig. 29) doit appartenir lui aussi à la première moitié, ou au plus tard au milieu du IVe s. En effet, la sépulture contenait également deux cratères attiques à figures rouges du "Peintre de Filottrano", datables autour de 350 et qui fournissent probablement la datation du contexte funéraire ${ }^{71}$. L'anse de Vienne $\left(n^{\circ} 7\right)$ est proche de celles du bassin de Filottrano et pourrait ainsi dater du milieu du IVe s. 

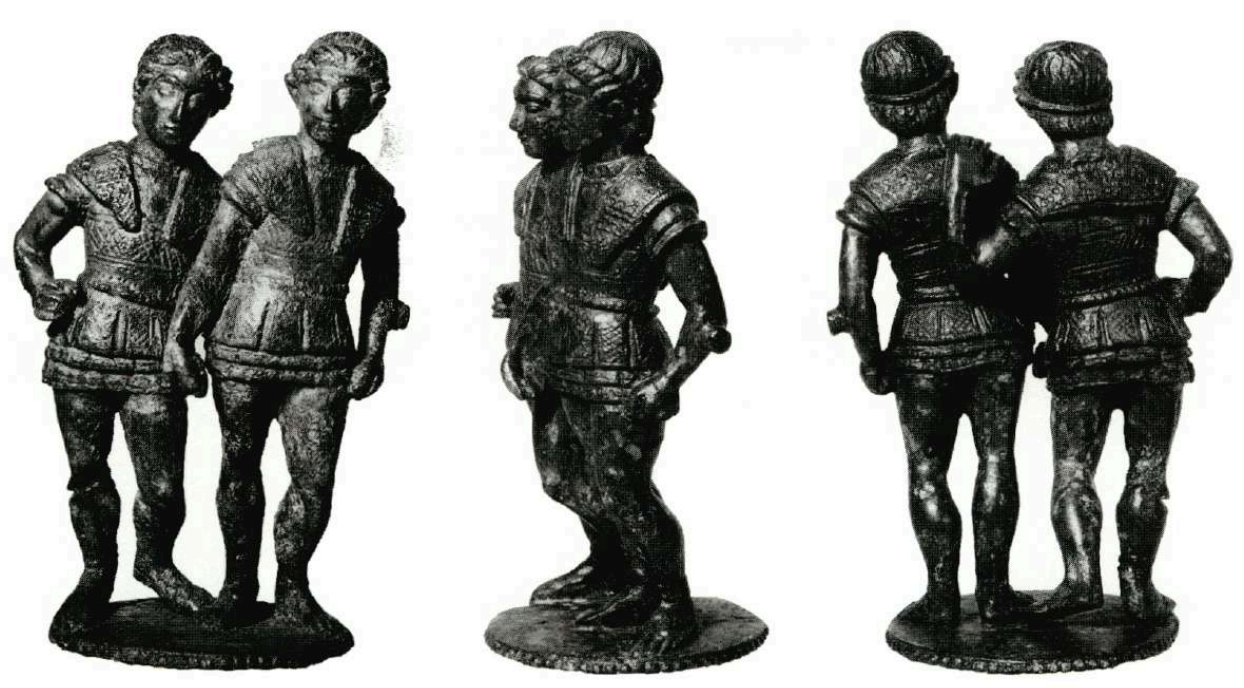

35-Cimaise de candélabre avec deux guerriers. Romisch-Germanisches Zentralmuseum, Mainz (Inv. $n^{\circ}$ 0.8919). Cl. V. Iserhardt, RGZM

C'est également à partir de 350, ou peu après, que nous proposons de situer les anses de Verna, en particulier à cause du traitement des chevelures, plus "floues" que dans les exemplaires précédents. Il est vrai que l'analyse stylistique des bronzes de Verna est rendue difficile par l'état de conservation de l'ensemble, mais le style des figurines, si on le compare au reste de la série, paraît s'écarter un peu plus encore des schémas tardo-classiques en vigueur en Etrurie jusqu'à la première moitié du IVe s. On peut les rapprocher des deux guerriers des couronnements de candélabres de la collection Sarti et du Musée de Mayence (supra, note 70) (fig. 35), qui manifestent de la même façon une fusion des traditions classiques avec des éléments nouveaux (là aussi perceptibles notamment dans le traitement des chevelures). On peut dater les deux cimaises vers 350 au plus tard.

En ce qui concerne le fragment d'anse trouvé en Anjou au milieu du XIXe s. (n 9) (fig. 36), l'aspect émoussé de certains de ses reliefs, notamment le visage du personnage, l'a fait parfois soupçonner d'être un "surmoulage", mais cela provient sans doute en partie des restaurations successives, et parfois brutales, auxquelles l'objet a pu être soumis depuis plus d'un siècle et demi ${ }^{72}$ Nous y verrions volontiers l'exemplaire le plus récent de toute la série, postérieur en tout cas à 350 et proche de certaines poignées de cistes prénestines, qu'on attribue en général à la seconde moitié du IVe s.

Il est intéressant, en effet, de comparer l'évolution de nos anses à lutteurs avec celle des poignées des cistes prénestines qui, au cours du IVe s., présentent de plus en plus un schéma proche des poignées de podanipteres, mettant aux prises deux lutteurs aux bras entrecroisés. Un exemplaire ancien (probablement première moitié du IVe s.) est fixé sur le couvercle d'une ciste à corps ajourép ${ }^{73}$. D'autres, qui correspondent à des cistes à décor gravé, datent au plus tôt de 350 , ou de la seconde moitié du siècle, et certains sont assez proches par le style des personnages des anses de Verna (poignées avec lutteurs masculins nus ${ }^{74}$ ). On peut d'ailleurs se demander si la composition des groupes de lutteurs de nos bassins a influencé la mise au point du schéma récurrent sur les poignées de ciste. Il a été en tout cas déjà souligné à quel point la production plastique des ateliers bronziers prénestins était redevable d'influences provenant d'Etrurie septentrionale et padane ${ }^{75}$. 

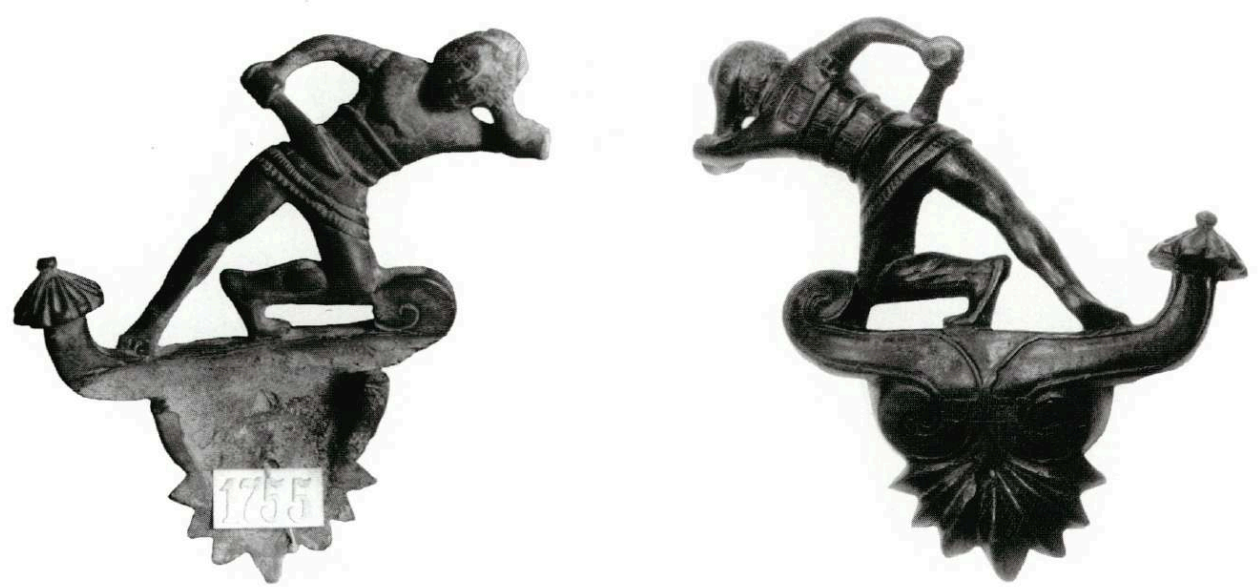

36-Anse de bassin à lutteurs : Sainte-Gemmes (Maine-et-Loire)

Pour résumer ces considérations sur la situation chronologique des bassins aux lutteurs, on peut souligner que ce type de récipient semble occuper une place particulière au sein de la production de vaisselle en bronze étrusque, à la fois par son caractère relativement exceptionnel et par la longévité du modèle : héritière d'une tradition venue de la production grecque, par l'intermédiaire de l'artisanat campanien, sa fabrication paraît connaître un nouvel essor à la fin du Ve s. et pendant une partie du IVe s., sans que l'on puisse reconstituer le cheminement qui a pu relier, au cours du Ve s., le bassin de Boston au reste des exemplaires, même si la filiation ainsi mise en évidence entre ateliers campaniens et ateliers d'Etrurie interne, septentrionale et padane, ne doit pas surprendre et connaît au cours du Ve s. d'autres illustrations.

\section{LE BASSIN DE VERNA DANS LE CONTEXTE DES IMPORTATIONS}

Une autre particularité de ce groupe est la proportion importante d'exemplaires découverts dans des contextes nord-alpins (fig. 31), d'autant plus que la date de fabrication que nous avons assignée à plusieurs d'entre eux (jusqu'à la seconde moitié du IVe s.), en fait une catégorie exceptionnelle parmi les importations de vaisselle de bronze étrusque en milieu celtique. On constate, en effet, à partir de la fin du Ve s., un net ralentissement du flux, qui était caractérisé, en particulier, depuis plusieurs décennies, par l'arrivée de Schnabelkannen, de stamnoi et de plats avec ou sans anses.

On rencontre encore, dans les dernières années du Ve s. et au début du IVe s., quelques pièces intéressantes pour leur rareté, parmi lesquelles on peut placer le bassin de Borsdorf et peut-être celui dont une seule palmette a été récemment retrouvée à Barzan, Le Fâ, en Charente-Maritime ( $n^{\circ} 10$ de notre liste) ${ }^{76}$ (fig. 37), sur un site qui a livré de façon diffuse du matériel de La Tène Ancienne. La datation stylistique du bronze de Borsdorf peut fort bien correspondre à celle de son contexte de découverte, malheureusement inconnu : les récentes et spectaculaires découvertes du tumulus du Glauberg ${ }^{77}$ rendent plausible la présence dans ce secteur, à proximité du site de hauteur fortifié, d'autres tombes fastueuses datables au tournant de La Tène A et de La Tène B, dont l'une aurait pu contenir le bassin étrusque.

Peu d'autres récipients ont été découverts dans des contextes postérieurs à 400 avant J.-C. Un kyathos de bronze provient du tumulus 9 de la nécropole de Bescheid "Bei den Hübeln", près de Trèves. Chronologiquement, la tombe se situe à la transition des 
phases Hunsrück-Eifel-Kultur II A 2/IIA 3, c'est-à-dire en chronologie absolue, vers 370. Mais le kyathos, qui appartient à une série bien attestée en Etrurie padane (Bologne, Sasso Marconi, Spina) dans la seconde moitié du Ve s. ${ }^{78}$ est antérieur d'environ un demi-siècle à la date de la déposition. On doit supposer une conservation de l'objet pendant environ deux générations, et sa déposition tardive montre que de tels objets conservaient encore tout leur prestige au début du IVe s. objets conservaient encore tout leur prestige au début du IVe s. C'est pour l'instant le seul récipient de ce type découvert en contexte assuré au nord des Alpes. Un autre de même forme (mais dépourvu de tout décor, alors que la plupart des kyathoi du "type Bologne" portent une ou plusieurs bandes d'un décor de tresse gravé) est conservé au Musée Denon de Chalon-sur-Saône : il proviendrait de Pourlans (Saône-et-Loire) sur la rive droite du Doubs (Guillaumet, Szabó 1985 : 71-72, fig. 1). L. Husty date ce vase, de manière globale, dans la deuxième moitié du Ve s., mais on ne peut préciser davantage et le contexte de la découverte est inconnu.

91 En revanche, l'oenochoè à bec de Saint-Etienne-de-Chigny (Indre-et-Loire) ne peut figurer dans cette liste, comme on l'a parfois suggéré ${ }^{79}$ Même si la forme du vase ("forme VI" de Beazley) est encore connue dans la production étrusque jusqu'au IIIe s., elle apparait dès 500, et le personnage en course agenouillée qui orne la cruche de Touraine incite à placer ce vase dans la première moitié du Ve $s .^{80}$ La forme est effectivement exceptionnelle en milieu celtique, mais cela ne suffit pas à indiquer une importation récente.

En dehors des bassins aux lutteurs, et dans l'état actuel de nos connaissances, les quelques récipients en bronze méditerranéens parvenus au nord des Alpes aux IVe s. et IIIe s. sont d'origine grecque et non pas étrusque. Le plus fameux d'entre eux est la situle de Waldalgesheim qui date du troisième quart du IVe s. Ce vase a été longtemps attribué à un atelier de Grande-Grèce, voire de Campanie, et cette attribution figure encore dans la récente publication de la tombe de Waldalgesheim ${ }^{81}$ Pourtant, depuis plusieurs années, $C$. Rolley et B. Shefton ont réexaminé parallèlement la situle et conclu que ce vase, plutôt que d'Italie, est originaire de Grèce propre (origine "égéenne" pour B. Shefton ${ }^{82}$ ou de Macédoine selon C. Rolley ${ }^{83}$ ). En revanche, ces deux auteurs ne s'accordent pas en ce qui concerne une autre situle de la même catégorie trouvée elle aussi en pays nordique : celle de Keldby, dans l'île de Møn, au Danemark (Copenhague, Musée national). B. Shefton (1994: 592-593) l'intègre à sa liste des produits du "Waldalgesheim Group", tandis que C. Rolley, qui la rapproche de deux situles de Florence, semble la considérer comme un produit italique.

C'est sans doute encore à la Grèce proprement dite qu'il faut attribuer une oenochoé à embouchure tréflée, dépourvue de son anse, qui faisait partie de la masse de bronzes pillés par les Alamans au IIIe s. de notre ère, dans des sanctuaires ou des villas romains de Rhénanie et retrouvés dans le Rhin à Neupotz ${ }^{84}$. Le type, dont la diffusion est large, de l'Italie à la Thrace, a été fabriqué vers la fin du IVe s. ${ }^{85}$ Le contexte originel de l'objet est bien sûr inconnu ; on a supposé qu'il pouvait se trouver encore à l'époque romaine conservé dans un sanctuaire, jusqu'au pillage de celui-ci par les Alamans ${ }^{86}$. 


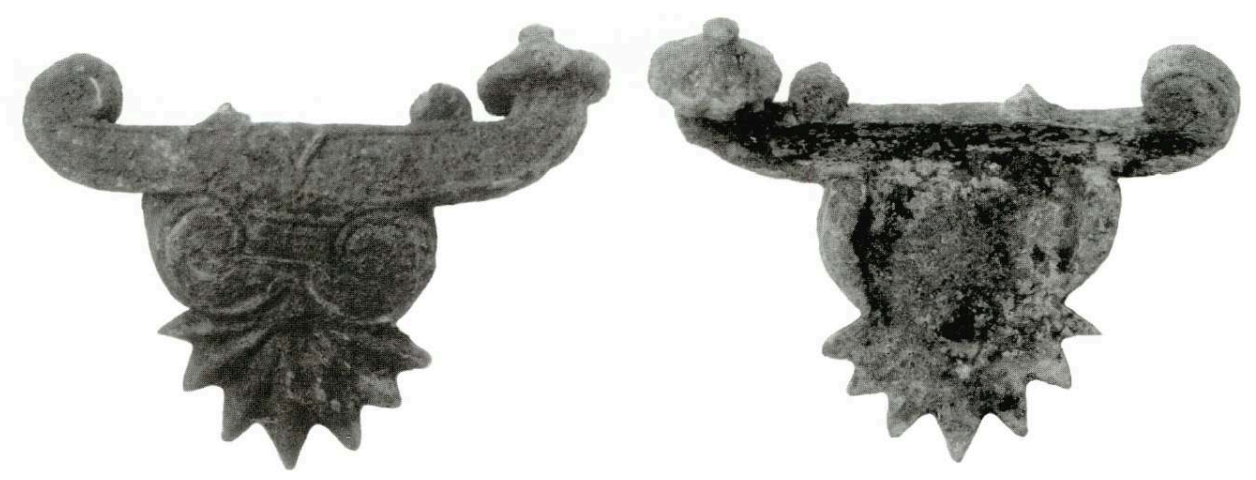

37-Anse de bassin à lutteurs Barzan (Charente-Maritime) des relations transalpines, les courants d'importation, qui se réduisent très sensiblement au IVe s., surtout avec l'Italie centrale, tandis que les quelques objets méditerranéens sont en majorité d'origine grecque. Sans doute même n'est-il plus très approprié de parler de "courant d'importation": les différents objets sont plus 
vraisemblablement parvenus au nord des Alpes de façon aléatoire et dispersée, à la faveur de conditions historiques nouvelles, y compris les plus récents des bassins aux lutteurs, même si ce modèle est attesté plusieurs fois hors d'Italie.

On a, à propos des objets grecs, évoqué la possibilité d'une arrivée "dans les bagages" de mercenaires (Morel 1990 : 281). Les rares objets originaires d'Italie ne seraient pas non plus liés à un circuit d'échanges de type "commercial", mais plutôt à la circulation d'individus entre le nord des Alpes et l'Italie celtique ${ }^{89}$. Malgré une situation géographique favorable qui lui assure une prospérité certaine pendant toute la protohistoire, on remarque d'ailleurs que la région de Verna n'a jamais été concernée auparavant par la diffusion de la vaisselle en bronze (alors que pour les régions de France occidentale qui ont fourni d'autres exemplaires de nos bassins, les courants d'importation d'objets en bronze originaires d'Italie semblent mieux attestés depuis le début de l'âge du Fer). Seule une coupe cannelée étrusque du VIIe s. av. J.-C., conservée à Lyon et peut-être d'origine locale, pourrait se rattacher à ce secteur, antérieurement à l'arrivée du bassin. Les importations de céramiques méditerranéennes, notamment attiques, intéressant la région lyonnaise, doivent participer d'un autre courant, qui utilise la voie rhodanienne (Perrin, Bellon 1992).

Peut-on, en conséquence, considérer le bassin de Verna comme un objet de prestige rapporté par un lointain Allobroge revenant d'Italie? Les élites celtiques d'Italie connaissaient ce type d'objets, comme le prouve le bassin de Filottrano.

Pour une période à peine plus récente (fin du IVe s. ou début du IIIe s.), les premières céramiques campaniennes trouvées au nord des Alpes ont été interprétées comme le signe possible de mouvements de personnes entre l'Italie et le Bassin Parisien ou la Suisse ${ }^{90}$ et même plus précisément du retour "au pays" de guerriers ayant opéré en Italie. Des fragments de céramiques à vernis noir anciennes sont signalés aussi dans la région lyonnaise Les mercenaires connus sous le nom de Gésates, recrutés par ${ }^{91}$. les Insubres et les Boïens contre Rome au IIIe s., étaient originaires, selon Polybe $(2,22)$, des Alpes et de la vallée du Rhône. Selon Strabon $(5,1,6)$, ces Gésates interviennent plus tôt dans les mouvements transalpins, puisqu'ils auraient accompagné les Sénons, lors de l'attaque de Rome au début du IVe s. Ces mouvements contribuent-ils à expliquer la présence dans la région d'objets d'origine italique aux IVe-IIIe s. ?

Pour le bassin de Verna, une autre donnée vient compliquer l'interprétation: le décalage entre la date de fabrication du bassin et celle de son dépôt dans la sépulture. Cela pose d'ailleurs la question du moment où l'objet est arrivé d'Italie. Compte tenu des réflexions précédentes, il est plus probable que cette date d'arrivée est de peu postérieure à celle de la fabrication. Le récipient, rapporté par un "ancêtre", aurait alors été conservé plusieurs générations avant sa déposition funéraire. Le cas n'est pas isolé, comme nous le verrons plus loin.

Mais on ne peut exclure totalement une arrivée plus récente. Certains objets d'origine étrusque ou italique semblent avoir fait l'objet d'une circulation tardive en Gaule, comme objets de "collection" ou d'“antiquariat". L'hypothèse a été avancée en particulier pour expliquer la présence d'un nombre non négligeable de petits bronzes votifs italiques sur des sites connus surtout à l'époque gallo-romaine (Adam $1992: 22$ ). Un miroir étrusque du IIIe s. av. J.-C. faisait partie du mobilier d'une sépulture parisienne du IIe s. de notre ère ${ }^{92}$ et deux fibules d'or étrusques du VIe s av. J.-C. ont été retrouvées à Saint-Aignan-de-Granlieu, près de Nantes, au sein d'un trésor monétaire du Ier s. de notre ère ${ }^{93}$. 


\section{LE BASSIN DANS LE CONTEXTE DE LA SÉPULTURE}

103 Si le bassin de Verna est plutôt, comme nous le supposons, un objet de prestige longtemps conservé en milieu celtique avant son dépôt dans la sépulture aristocratique, son exemple n'est pas totalement isolé, même si dans ce cas le décalage chronologique est exceptionnellement important. Les écarts, en effet, dans la plupart des cas, n'excèdent guère la durée d'une ou deux générations, par exemple dans la tombe princière de Vix, où le cratère doit être de 20 à 30 ans plus ancien que les coupes attiques et le matériel indigène. Le bassin étrusque à ombilic de la même tombe est lui aussi antérieur de quelques décennies à la date de la déposition. Pour le même secteur du Mont Lassois, on constate un écart un peu plus important dans le tumulus de La Garenne à Sainte-Colombe, où le trépied étrusque, fabriqué dans la première moitié du Vie s., est associé à des objets de parure locaux caractéristiques du Hallstatt D3 ${ }^{94}$.

On peut ainsi multiplier les exemples : l'hydrie de Grächwil, datée vers 600 , est associée à un char de la fin du VIe s; le trépied étrusque de Dürkheim, dans le Palatinat, est antérieur d'une ou deux générations à la déposition funéraire qui le contenait. Dans la tombe princière de Grafenbuhl, près de Ludwigsburg, dans le Wurtemberg, les objets importés, d'origine diverse, couvrent une période qui va de la fin du VIIe s. (pour le manche de miroir en ivoire) à la seconde moitié du VIe s., pour la klinè grecque, tandis que l'enfouissement définitif est daté, là encore par les objets de parure indigènes, au début du Ha D3 (vers 500 av. J.-C.) ${ }^{95}$ On a constaté que ces objets plus anciens sont, en général, des pièces exceptionnelles et d'origine lointaine et on a proposé de voir dans le phénomène $\mathrm{de}^{96}$ thésaurisation de ces biens de prestige (keimèlia) un trait caractéristique de la mentalité aristocratique qui affirme, par la consécration de précieux objets "de famille", la continuité de la lignée ${ }^{97}$. Certains cas d'antériorité d'une pièce $d u$ mobilier par rapport au reste $d u$ contexte recouvrent également un remaniement de sépulture (par exemple dans la tombe de Bouranton, dans l'Aube, où le chaudron de bronze contenait une sépulture à incinération antérieure à la déposition principale). Cette pratique n'est peut-être pas étrangère, non plus, à la volonté de mettre en évidence un lignage familial.

Tous les exemples précédemment cités se réfèrent à l'époque hallstattienne et à La Tène Ancienne. La situation que nous rencontrons à Verna paraît beaucoup plus exceptionnelle pour la fin de La Tène. En dehors de deux sépultures Scandinaves, datées vers 100 av. J.-C. et renfermant chacune un récipient en bronze d'origine italique et nettement plus ancien ${ }^{98}$, aucun autre cas en milieu proprement celtique ne nous est connu pour l'instant.

Du fait de sa propre chronologie et de la datation du contexte archéologique dont il provient, le bassin aux lutteurs de Verna constitue donc à bien des égards une rareté. On peut se demander, pour terminer, si les Gaulois qui l'ont déposé dans la sépulture l'ont considéré du seul point de vue de son statut de keimèlion, bien de prestige que sa haute antiquité rendait plus précieux encore, ou s'ils l'ont intégré au service funéraire, lui restituant en quelque sorte sa fonction originelle de récipient à ablutions, en lieu et place d'un bain de pieds plus' "moderne" (comme les grands bassins de type Eggers 94) et en association avec d'autres récipients présents dans la sépulture, comme la cruche de type Kelheim et les patelles à manche de "type Aylesford". 


\section{CONCLUSION. M. Schönfelder}

107 La vaisselle en bronze de Verna représente le plus grand ensemble connu pour une tombe de La Tène finale en France. Si l'on se réfère à la documentation de 1818, la majorité de la vaisselle appartient à la tombe à char : la grande situle, la cruche, le couvercle (avec une autre cruche?), une ou deux patelles à manche. La petite situle qui est entière et une anse étrusque sont figurées également sur une planche d'Artaud. De même que le bassin rectangulaire, le bassin à bec verseur et le bassin étrusque, il est fort probable qu'ils fassent partie de la sépulture. Ils ont été fondus et détruits rituellement de la même manière que les autres vases. Ces récipients étant de formes rares, ils appartiennent certainement à un grand ensemble, plutôt que d'être les uniques récipients de tombes secondaires. Quelques objets ne sont pas identifiés dans la documentation ancienne, comme le fragment de la deuxième petite situle ou le gobelet de type Idria, mais l'état de conservation en général laisse penser qu'ils font également partie de l'ensemble.

108 L'attribution d'une fonction à un type de vase comporte toujours le problème fondamental d'une utilisation différente entre le lieu de production et celui de l'utilisation finale. Si les services de vaisselle ont été créés au départ pour des usagers (italiens) d'un certain niveau social, on ne connait pour les Celtes que leur utilisation dans les tombes. De plus, leur présence dans ces tombes dépend des courants d'importation : le dépôt funéraire est l'ultime destination régie par d'autres règles que celles qui présidaient aux usages de ces récipients. Les rites funéraires locaux sont dominants pour le choix des vases comme nous l'avons déjà évoqué. Reste enfin le problème de la dénomination moderne des vaisselles antiques, qui comporte déjà une fonction implicite.

109 L'étude des dimensions de différents récipients de Verna (tab. 2, 5 et 6) montre que les vases destinés à la tombe sont tous plus grands que la plupart de ceux des autres contextes connus. Ces choix sont peut-être à mettre en relation avec une position sociale très élevée du défunt. Cette observation permet de porter un regard différent sur ce qui peut caractériser un statut élitiste, par rapport à la conception traditionnelle basée uniquement sur le nombre des objets.

110 La majorité des vases de Verna appartiennent à des types républicains connus. La fonction de plusieurs types demeure cependant incertaine (tab. 8). De plus, il est étonnant que la passoire et le simpulum soient absents ; il est possible qu'on ait renoncé à ces deux instruments de la cérémonie du vin du rituel funéraire comme pour les régions du Nord de la France. Par contre en Provence, les simpula sont les ustensiles les plus fréquemment utilisés dans le rite funéraire (Schönfelder 2000a: 365 fig. 234; Schönfelder sous presse). En Allemagne, dans la région autour de Francfort et Mayence, ce n'est pas un hasard si on trouve des passoires ${ }^{99}$ dans plusieurs tombes ; la présence des vases en bronze y est le reflet de rituels funéraires locaux.

111 L'ensemble de Verna est un des plus grands de Gaule et il appartient à un complexe funéraire également très riche. Cependant, il ne compte pas de vases en métaux précieux ou en verre, comme dans d'autres tombes du Tessin ${ }^{100}$, ainsi que dans quelques dépôts et épaves, comme par exemple le simpulum et les passoires en argent de Mengibar (Prov. Jaén, E) (Raddatz 1969 :92-93 ; pl. 24,2-3.6) et les puisoirs des trésors de Arcisate (Prov. Varese, I), de Tivoli (Prov. Roma, I) ou de l'épave de Cap Taillat (dép. Var) ${ }^{101 .}$ Ceci montre qu'on a aussi fabriqué les types. les plus courants en argent qui ne 
sont, en général, pas déposés dans les tombes. Des vases en argent de tradition hellénistique, comme des kantharoi, kyathoi et des bols à pied ne sont pas connus dans les tombes et les dépôts laténiens. L'exception des trois vases de la Saône à Thorey (Saône-et-Loire) peut s'expliquer par les circonstances particulières d'un accident sur la Saône (Baratte 1989 ; Bonnamour 1989). Dans la vie quotidienne, les indicateurs de prestige chez les "aristocrates" celtes riches étaient plutôt - comme l'affirment les sources antiques - ces vases en métaux précieux. Malgré ces restrictions, on a besoin d'utiliser la vaisselle en bronze dans les tombes comme indices de hiérarchie sociale. Il faut aussi remettre ces objets dans le contexte des rites funéraires locaux. On suppose donc qu'il s'agit dans le cas de Verna de la tombe d'un défunt situé au sommet de la hiérarchie sociale, le rituel d'ablution et le rituel du vin étant présents. Enfin, les vases sont plus grands que les autres, et il existe des formes rares et anciennes.

\begin{tabular}{|l|l|l|l|}
\hline \multicolumn{1}{|c|}{ Groupe de fonction } & \multicolumn{1}{|c|}{ Terme fonctionnel } & \multicolumn{1}{|c|}{ Terme traditionnel } & \multicolumn{1}{c|}{ Types / Exemples } \\
\hline Ablutions & récipient à rincer & cruche & type Kelheim (type Idria) \\
\hline & $\begin{array}{l}\text { bassin de réception } \\
\text { (lavage des mains) }\end{array}$ & patelle à manche, poêlon & type Aylesford \\
\hline & $\begin{array}{l}\text { bassin de réception } \\
\text { (lavage des pieds) }\end{array}$ & bassin à pied & $\begin{array}{l}\text { grand bassin à pied avec attaches à feuilles de } \\
\text { vigne (type Eggers 94) }\end{array}$ \\
\hline Service à vin (à boire) & récipient à eau & grande situle & type Beaucaire ( ?) \\
\hline & récipient à vin & petite situle & types Eggers 21-23 \\
\hline & récipient à vin mélangé & grande situle / cratère & type Beaucaire ( ?), types Eggers 18-19 \\
\hline & puisoir & simpulum & type Pescate, simpula à manche vertical \\
\hline & passoire & passoire & type Guillaumet \\
\hline & gobelet à verser & gobelet à anse & type Idria \\
\hline & gobelet à boire & gobelet hémisphérique & Ornavasso \\
\hline & bassin à bec verseur & bassin à bec verseur & Wiebendorf \\
\hline & écuelle à manger & écuelle plate & Châtillon-sur-Indre \\
\hline Service à manger & bassin à servir & bassin à anses mobiles & Clémency \\
\hline & bassin à servir & bassin rectangulaire & Verna \\
\hline & chaudron & chaudron & types Eggers 4-8 \\
\hline & bassin à bec verseur & bassin à bec verseur & Wiebendorf \\
\hline Autre & & & \\
\hline & & & \\
\hline & & &
\end{tabular}

Tableau 8 -Propositions de fonctions pour la vaisselle en bronze républicaine

\section{LES PANOPLIES GUERRIÈRES. M. Schönfelder avec une contribution de $B$. Herbold}

D'après le rapport de fouilles de 1818 , la fosse de la tombe centrale contenait deux épées et un casque. Puis dans un commentaire ajouté dans une copie du manuscrit originel ( $c f$. annexe 1), ce sont trois épées pliées qui sont signalées, deux avec leurs fourreaux en fer, une autre avec un fourreau en bronze. L'attribution à cette sépulture de trois umbos et de six pointes de lances supplémentaires qui sont conservés, dans la collection de Verna n'est pas certaine, mais possible, le rapport de 1818 mentionnant "plusieurs armes" alors qu'un umbo et trois lances figurent sur les planches anciennes (fig. 38).

Le casque de la tombe, décrit comme "un casque de cuivre sans ornement", n'est pas documenté. Il était dessiné sur une planche ( $c f$. annexe $1:$ Pl. II. P), qui reste également introuvable. Il faut souligner que ce casque n'a pas été confondu avec une pièce de vaisselle en bronze, celle-ci ayant été reconnue par les fouilleurs. La description trop brève ne révèle pas d'indices sur la forme et le type de ce casque, qui permettraient de 
savoir si on a affaire à un casque de tradition romaine ${ }^{102}$ ou à un casque celtique en bronze ${ }^{103}$. Quoi qu'il en soit, la présence d'un casque valorise la tombe de Verna, par comparaison avec d'autres tombes de guerriers.

\section{LES ÉPÉES}

114 A ce jour, les fragments de cinq épées avec leurs fourreaux sont attribués à Vema. Tout d'abord, le rapport de 1818 mentionne et figure deux épées ( $n^{\circ} 1$ et 2 ) qui font partie de la sépulture (cf. annexe 1) (fig. 4, objets AA, BB). Une autre épée, présente sur la planche (fig. 4, objet KK) est une comparaison, qui provient du cimetière de Servanes à Mouriès (dép. Bouches-du-Rhône) ${ }^{104}$; cette planche est probablement l'une des premières, dans l'histoire de l'archéologie, qui propose une figure de comparaison. Puis dans le commentaire, trois épées sont mentionnées: "les trois sabres que cette fouille a produits [sic], étaient tous repliés en trois parties, et aux fragments de leurs fourreaux on a pu reconnaître que deux de ces fourreaux étaient enfer, et l'autre en cuivre". Cette phrase mentionne donc une épée à fourreau en bronze, sans renvoi à une planche, et que l'on trouve uniquement dans ce texte. S'il existe bien une épée avec un fourreau en fer et en bronze $\left(n^{\circ} 3\right)$, ainsi que des petits fragments d'un autre fourreau en bronze, l'attribution des épées au tumulus de Verna n'est pas absolument claire. La grande épée pliée (fig. 5, objet I) qui peut correspondre à l'épée au fourreau en bronze et fer $\left(\mathrm{n}^{\circ} 3\right)$ provient probablement de cette fouille de Verna, mais peut-être pas de la tombe centrale. Quant à l'autre épée de cette planche (fig. 5, objet VIII) qui n'a aucun lien direct avec le texte et les matériaux indiqués, sa provenance reste douteuse. Dans la collection de Verna, on trouve également une quatrième lame d'épée $\left(n^{\circ} 4\right)$ et un petit fragment appartenant à une autre épée $\left(n^{\circ} 5\right)$. Ce dernier élément n'a peut-être pas été identifié à l'époque.

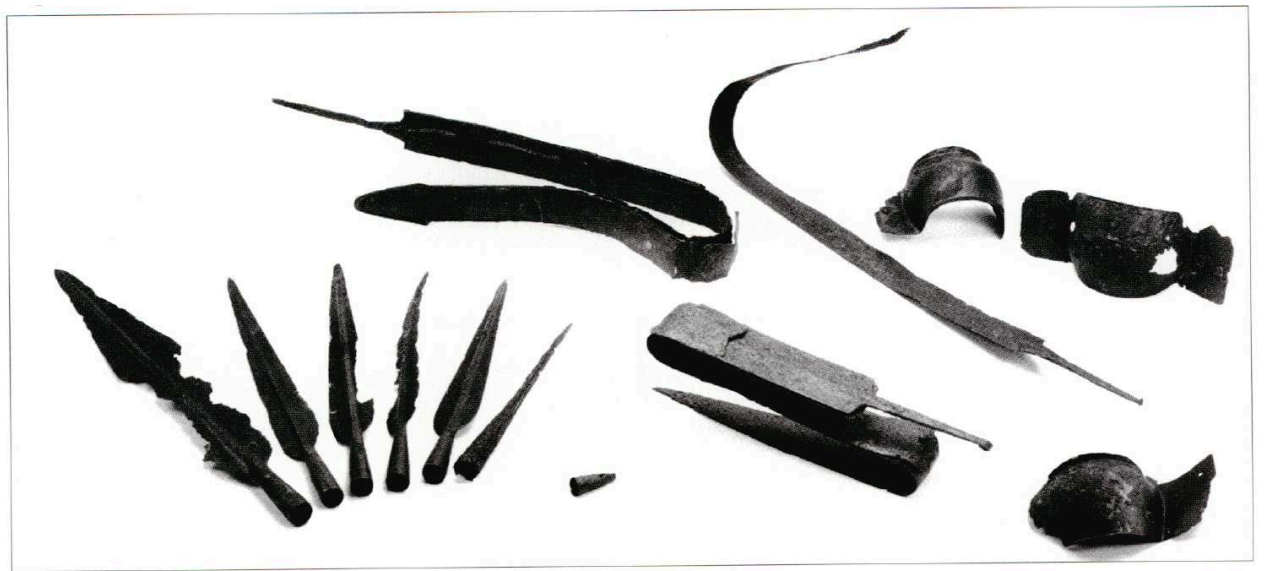

38-Une partie de la lie guerrière de Verna

La première épée, dans son fourreau en fer, pliée deux fois (fig. 39) a pu être identifiée sur une planche ancienne (fig. 4, objet AA). Le rapport indique "l'une fixée dans son fourreau est repliée en trois parties". Son fourreau est aujourd'hui lacunaire : la bouterolle dessinée sur la planche ancienne a disparu, et il ne subsiste qu'un fragment caractéristique de la gouttière et la pièce de suspension. L'épée et son fourreau conservent les traces du feu du bûcher. Quelques éclats sur la partie inférieure de la lame témoignent d'une tentative récente de la redresser. D'après la terminologie 
actuelle, le fourreau peut être identifié comme un fourreau en fer de type Ludwigshafen.

116 L'épée (long, totale $89 \mathrm{~cm}$ ) a une lame plate (larg. $6 \mathrm{~cm}$ ) sans nervure, avec une pointe triangulaire d'environ $13 \mathrm{~cm}$ formant un angle à $20^{\circ}$. L'extrémité de la soie (long. 11,3 $\mathrm{cm}$ ) à section rectangulaire fine est courbée et forme un petit anneau. Le passage entre la soie et la lame est rectangulaire.

Les fourreaux de type Ludwigshafen appartiennent aux armes de La Tène ${ }^{105}$ les plus soumises à discussion.

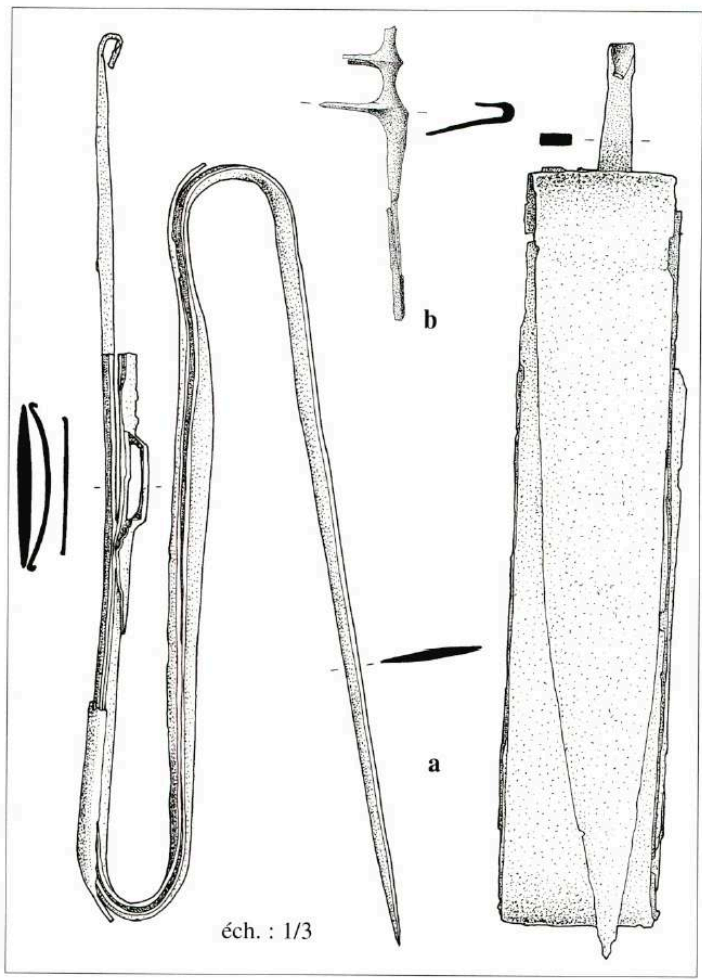

39-Épée à fourreau de type Ludwigshafen (fer)

\begin{tabular}{|l|l|l|l|l|l|l|l|}
\hline $\begin{array}{l}\text { Critères } \\
\text { possibles }\end{array}$ & Dimensions & Matériau & Entrée & $\begin{array}{l}\text { Pièce de } \\
\text { suspension }\end{array}$ & $\begin{array}{l}\text { Partie haute } \\
\text { de la bouterolle }\end{array}$ & Bouterolle & $\begin{array}{l}\text { Extrémité de la } \\
\text { bouterolle }\end{array}$ \\
\hline $\begin{array}{l}\text { Solutions } \\
\text { possibles : }\end{array}$ & $\begin{array}{l}96,4: 4,7 \\
\text { (Ludwigs.) }\end{array}$ & $\begin{array}{l}\text { bronze } \\
\text { (Ludwigs.) }\end{array}$ & $\begin{array}{l}\text { fine, droite avec } \\
\text { agrafe droite } \\
\text { profilée } \\
\text { (Ludwigshafen) }\end{array}$ & $\begin{array}{l}\text { bronze, } \\
\text { rectangulaire, } \\
\text { traverses droites } \\
\text { au revers } \\
\text { (Ludwigshafen) }\end{array}$ & $\begin{array}{l}\text { 2 paires de griffes } \\
\text { /peu d'agrafes } \\
\text { doubles-S } \\
\text { (Ludwigshafen) }\end{array}$ & $\begin{array}{l}\text { en forme de bateau } \\
\text { - moins large } \\
\text { (Ludwigshafen) }\end{array}$ \\
\hline $\begin{array}{l}81: 6 \\
\text { (Pouilly) }\end{array}$ & $\begin{array}{l}\text { fer } \\
\text { (Alizay) }\end{array}$ & $\begin{array}{l}\text { fine, droite avec } \\
\text { agrafe droite } \\
\text { profilée et } \\
\text { agrafe double-S } \\
\text { (Pouilly) }\end{array}$ & $\begin{array}{l}\text { lenforcement en } \\
\text { fer, rectangulaire, } \\
\text { traverses droites } \\
\text { au revers } \\
\text { (Wederath 296) }\end{array}$ & $\begin{array}{l}\text { sans profil } \\
\text { (Wederath 296) }\end{array}$ & $\begin{array}{l}\text { 2 paires de griffes } \\
\text { jointes, traverses } \\
\text { droites } \\
\text { (Veringenstadt) }\end{array}$ & $\begin{array}{l}\text { en forme de bateau } \\
\text { - très large } \\
\text { (Heuchelheim) }\end{array}$ \\
\hline$\ldots$ & $\ldots$ & $\begin{array}{l}\text { fine en forme de } \\
\text { cloche, avec } \\
\text { agrafe droite } \\
\text { profilée } \\
\text { (Regensburg) }\end{array}$ & $\begin{array}{l}\text { bronze, } \\
\text { rectangulaire, } \\
\text { agrafes en forme } \\
\text { de double-D } \\
\text { au revers } \\
\text { (Heuchelheim) }\end{array}$ & $\begin{array}{l}\text { décorée avec } \\
\text { des peltes } \\
\text { (Veringenstadt) }\end{array}$ & $\ldots$ & $\ldots$ \\
\hline
\end{tabular}

Tableau 9 -Caractéristiques des fourreaux d'épées de type Ludwigshafen 


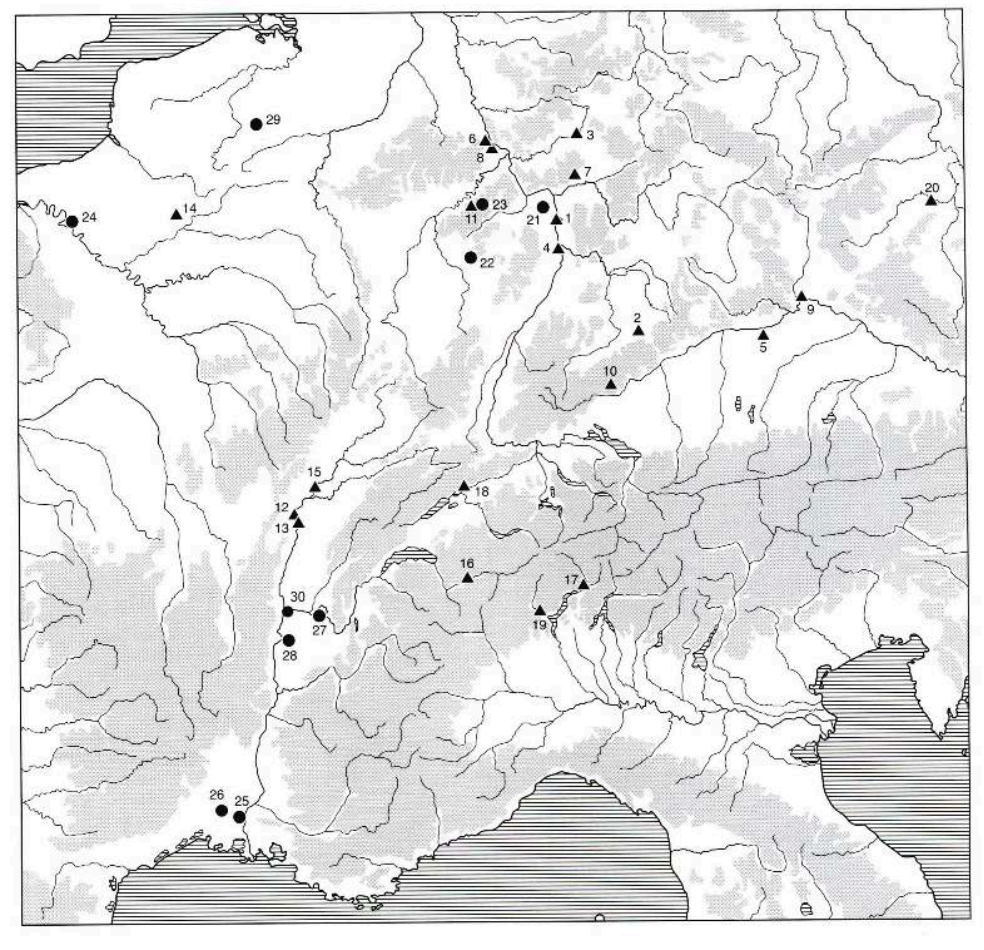

$\Delta$ Bronze

40-Répartition des fourreaux d'épée du groupe Ludwigshafen. Bronze : 1Eich. - 2 Göppingen. - 3 Heuchelheim. - 4 Ludwigshafen. 5 Manching. - 6 Neuwied/Wollendorf tombe 2.- 7 OberurselOberstedten/Heidetränke. - 8 Plaidt. - 9 Regensburg. - 10 Veringenstadt. - 11 Wederath tombe 296. 12 Chalon-sur-Saône. - 13 Lux. - 14 Montmartin. - 15 Pouilly. - 16 Conthey. - 17 Giubiasco tombe 96. 18 Port (3 exemplaires). - 19 Ornavasso/San Bernardo. - 20 Stradonice - Fer : 21 - Hahnheim. - 22 Mainzweiler. - 23 Wederath tombe 1192. - 24 Alizay. - 25 Beaucaire/Les Colombes, tombe 5. - 26 Nîmes/Camplanier. tombe 1.-27 Verna. - 28 Vienne. - (D'après Wieland 1996) - 29 Pommeroel - 30 Lyon (Saint-Vincent)

Les recherches de U. Schaaff montrent qu'en plus des exemplaires les plus fréquents en bronze, il existe des fourreaux en fer présentant les mêmes détails typologiques. U. Schaaff remarque une distribution dominante de ces exemplaires (Schaaff 1985: 623; Schaaff $1986: 295$ ) dans les régions occidentales (fig. 40).

Les fourreaux de type Ludwigshafen présentent de nombreuses caractéristiques qui permettent de définir différents critères typologiques. Ainsi, les pièces de suspension de schéma La Tène moyenne sur des fourreaux de Chalon-sur-Saône (dép. Saône-etLoire) et Port (canton de Bern, $\mathrm{CH}$ ) présentent une connexion typologique avec les fourreaux d'épées du groupe de La Tène moyenne ${ }^{106}$. Un exemplaire trouvé dans le Danube à Regensburg (Stadt Regensburg, D) avec une entrée en profil en cloche et un pontet peut-être de schéma La Tène moyenne permet aussi ce rapprochement. Par contre, la pièce de renfort de cet exemplaire est typique de La Tène finale ${ }^{107}$. Autre exemple, le fourreau de la tombe 131 de Mirandola (Prov. Verona, I) (Salzani 1996, pl. $60,18)$, comporte une bouterolle qui ressemble à celle de Ludwigshafen, dont l'extrémité haute est décorée avec une paire de têtes d'oiseaux, comme les bouterolles de schéma La Tène moyenne; toutefois sa partie supérieure se rapproche davantage du faciès de La Tène moyenne, ce qui ne permet donc pas de l'attribuer sans réserves au type de Ludwigshafen.

La variété des matériaux utilisés pour les pièces de suspension rectangulaires des fourreaux classiques de type Ludwigshafen, laisse supposer des ateliers différents ${ }^{108}$. 
L'agrafe à double-D sur l'autre face du fourreau pourrait permettre de le caractériser ${ }^{109}$, mais elle se rencontre aussi sur les fourreaux qui sont habituellement attribués au type Ormes $^{110}$. Les détails de la bouterolle autorisent encore d'autres classifications.

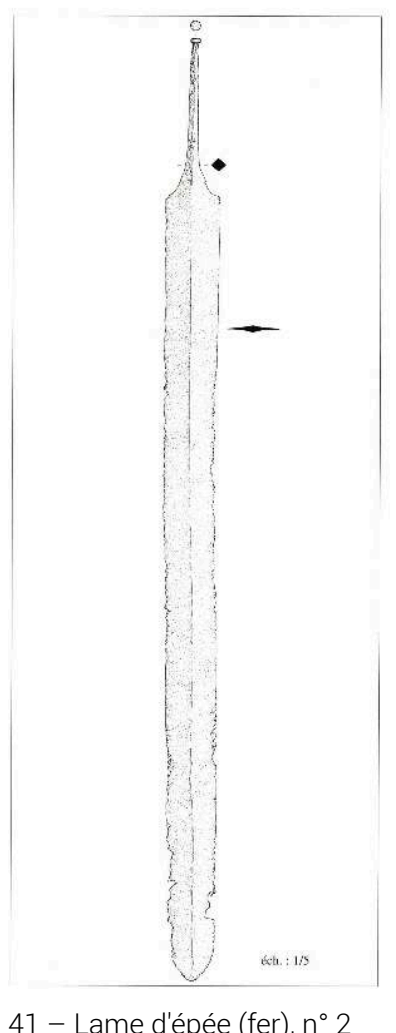

La diversité typologique des épées de La Tène finale est très vaste. Pour une discussion approfondie, il n'est guère possible de travailler avec l'ancien classement des différents types qui ne se réfère qu'à un seul fourreau pour caractériser chaque type. La totalité des critères de classification des fourreaux ne peut en effet être réunie sur un seul exemplaire (tab. 9). Seuls certains éléments, tels que la bouterolle, la pièce de suspension ou encore l'entrée du fourreau, permettent de définir des combinaisons de critères suffisamment cohérentes pour déterminer un "type". Pour les fourreaux de La Tène, il semble préférable de distinguer un "groupe Ludwigshafen" d'autres groupes de fourreaux. L'utilisation jusqu'à maintenant de la terminologie de "fourreaux de schéma La Tène ancienne, moyenne et finale" n'est pas un hasard. Elle exprime la difficulté à établir une typologie précise. Les analyses des fourreaux du sanctuaire de Gournay-surAronde (dép. Oise) par Th. Lejars (Lejars 1994 :17-38) suivent aussi cette idée pour La Tène $B$ et $C$.

Une deuxième épée a été illustrée (fig. 4, objet BB) et décrite comme non pliée dès 1818. Elle correspond à une lame $\left(\mathrm{n}^{\circ} 2\right)$, qui a été dessinée en 1986 (fig. 41), mais qui reste aujourd'hui introuvable. La longueur totale de cet objet est de $101,5 \mathrm{~cm}$, sa largeur de 5,8 à 5,3 cm. La lame à nervure centrale munie d'une pointe arrondie est formée par les quatre derniers centimètres et passe par une courbure à la soie à section rhombique. La soie se termine par un petit bouton. Pour une détermination typologique et chronologique de cette épée $n^{\circ} 2$, seules les mesures peuvent être utilisées. Si l'on se réfère aux études de F. Millier concernant les 
dimensions des épées des grands ensembles laténiens, la lame de Verna correspond très bien aux lames de l'ensemble fluvial de Port (Kt. Bern, $\mathrm{CH}$ ), daté de La Tène D1 ${ }^{111}$.

Une autre épée $\left(n^{\circ} 3\right)$ est pliée dans son fourreau en bronze et en fer de schéma La Tène moyenne évolué (fig. 42). Elle correspond à un dessin d'une planche ancienne (fig. 5, objet I), mais elle n'est pas mentionnée dans le texte. Le côté frontal en bronze a été éclaté plusieurs fois par le pliage, alors qu'il y a des traces de feu sur la bouterolle (long, total $102 \mathrm{~cm}$; larg. fourreau $4,3-4,7 \mathrm{~cm}$ ).

Le fourreau a une entrée en forme de cloche; sa face postérieure porte un pontet asymétrique (long. $18 \mathrm{~cm}$ ). La bouterolle à gouttières parallèles connaît un épaulement au niveau d'une paire de pattes de fixation et se termine en triangle. Son extrémité est un peu épaissie. Sur le côté frontal du fourreau, il existe une petite marque effectuée au repoussé qui correspond au sommet de la bouterolle. Cette petite marque est probablement un repère de montage qui permet de "clipser" la bouterolle au fourreau. Cette méthode est courante pour tous les fourreaux, notamment à partir de La Tène $\mathrm{C} 2^{112}$.

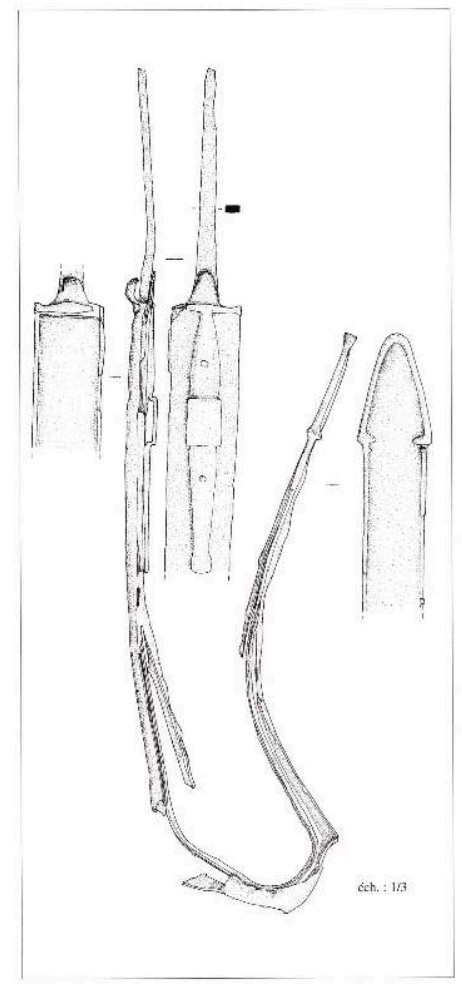

42 - Epée avec son fourreau (fer et bronze), $n^{\circ} 3$ 


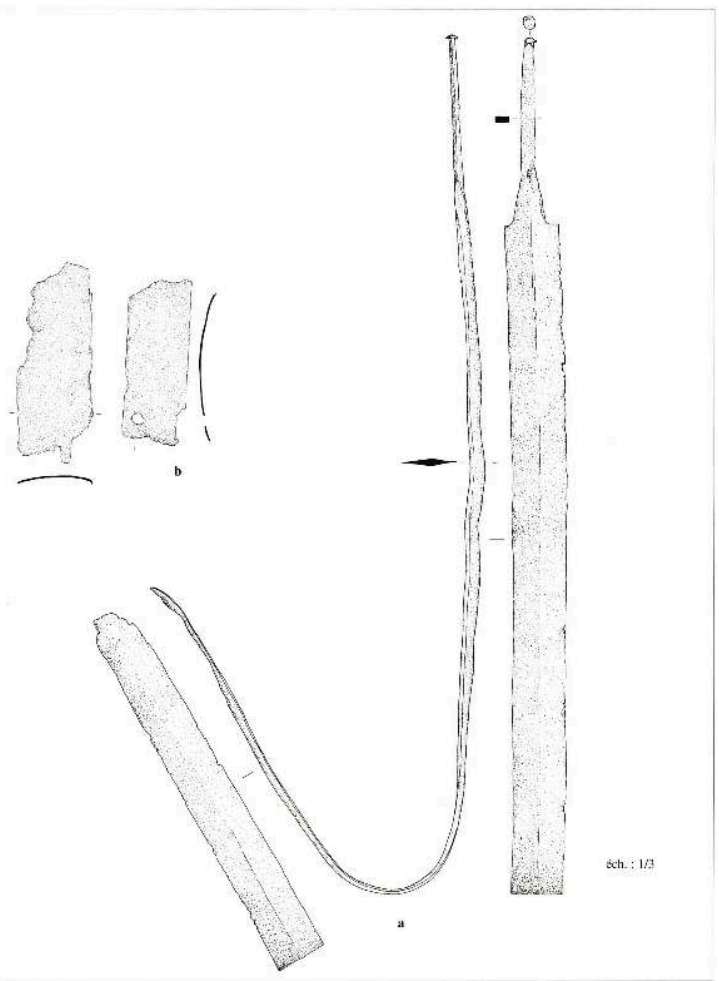

43 a - Lame d'épée $n^{\circ} 4 ; b$ - Fragments de son fourreau (fer)

\begin{tabular}{|c|c|c|c|c|c|c|c|c|c|}
\hline Lieu & $\begin{array}{c}\text { Long. totale } \\
\text { (cm) }\end{array}$ & $\begin{array}{l}\text { larg. flamme } \\
\text { (cm) }\end{array}$ & $\begin{array}{l}\text { Long. douille } \\
\text { (cm) }\end{array}$ & $\begin{array}{l}\text { Long. flamme } \\
\text { (cm) }\end{array}$ & $\begin{array}{l}\text { L. flamme : } \\
\text { 1. flamme }\end{array}$ & $\begin{array}{l}\text { L. flamme : } \\
\text { L. douille }\end{array}$ & Autres armes & Datation & Bibliographie \\
\hline $\begin{array}{l}\text { Nanterre, tombe à char } \\
\text { (Dép. Hauts-de-Seine, F) }\end{array}$ & 34,8 & 10,2 & 8,2 & 26,6 & $\begin{array}{c}2,6 \\
\text { flamme large }\end{array}$ & \begin{tabular}{|c|}
4,2 \\
douille longue
\end{tabular} & épée, 2: lance & Lt C2 & Hubert 1902. - M.A.N. \\
\hline $\begin{array}{l}\text { Koblenz-Arzheim, tombe } \\
\text { (Kr. Mayen-Koblenz, D) }\end{array}$ & 35,5 & 3,6 & 7,3 & 28,2 & $\begin{array}{c}7,8 \\
\text { flamme mince }\end{array}$ & 3,9 & épée, bouclier & $\mathrm{LtC} 2$ & $\begin{array}{l}\text { Schumacher 1999: } \\
140 \text { pl. } 67 \mathrm{~b} \text {. }\end{array}$ \\
\hline $\begin{array}{l}\text { Nìmes "Pissevin", tombe } 1 \\
\text { (Dép. Gard, F) }\end{array}$ & 38,2 & 3 & 8,5 & 29,7 & $\begin{array}{c}9,9 \\
\text { flamme mince }\end{array}$ & 3,5 & \begin{tabular}{|c|} 
tombe double \\
2 épées, \\
2 lances
\end{tabular} & Lt D1 & $\begin{array}{l}\text { Py } 1981: 151 \\
\text { fig. } 66,4 .\end{array}$ \\
\hline $\begin{array}{l}\text { Mépieu, tombes } \\
\text { (Dép. Isère, F) }\end{array}$ & 44,1 & 6,8 & 7,7 & 36,4 & 6,5 & 4,7 & $?$ & $\mathrm{LtC} 2$ & $\begin{array}{l}\text { Bocquet } 1991: 251 \\
\text { fig. } 2 \text { B5. }\end{array}$ \\
\hline $\begin{array}{l}\text { Wederath, tombe } 1178 \\
\text { (Kr. Bernkastel-Wittlich, D) }\end{array}$ & 44,4 & 8 & 7 & 37,4 & 4,7 & 5,3 & épé, bouclier & Lt Dla & $\begin{array}{l}\text { Haffner 1978: } \\
56 \text { pl. } 296,3 \text {. }\end{array}$ \\
\hline $\begin{array}{l}\text { Worms-Heppenheim, tombe } \\
\text { (Kr. Alzey-Worms, D) }\end{array}$ & 45 & 9 & 7.8 & 37.2 & 4,1 & 4,8 & épée & $\mathrm{LtC} 2$ & $\begin{array}{l}\text { Behrens } 1923 \text { : } \\
15 \text { fig. } 19 \text {. }\end{array}$ \\
\hline $\begin{array}{l}\text { Wederath, tombe } 2000 \\
\text { (Kr. Bernkastel-Wittlich, D) }\end{array}$ & 45,2 & 10,6 & 9,2 & 36 & \begin{tabular}{|c|}
3,4 \\
flamme large
\end{tabular} & \begin{tabular}{|c|}
3,9 \\
douille longue
\end{tabular} & épée, bouclier & LID2 & $\begin{array}{l}\text { Cordie/Haffner } 1997 \text { : } \\
\text { pl. } 543 \text { c. }\end{array}$ \\
\hline $\begin{array}{l}\text { Montmort, tombes } \\
\text { (Dép. Saône-et-Loire, F) }\end{array}$ & 45,5 & 8 & 8,1 & 37,4 & 4,7 & 4,6 & épée & Lt C2-Dla & $\begin{array}{l}\text { Guillaumet } 1979 \text { : } \\
196 \text { sq. pl.2. }\end{array}$ \\
\hline $\begin{array}{l}\text { Wederath, tombe } 1228 \\
\text { (Kr. Bernkastel-Wittlich, D) }\end{array}$ & 45,6 & 3,1 & 6,6 & 39 & $\begin{array}{c}12,6 \\
\text { flamme mince } \\
\end{array}$ & 5,9 & épée, bouclier & Lt DI & $\begin{array}{l}\text { Haffner } 1978 \text { : } \\
\text { pl. } 315,3 \text {. }\end{array}$ \\
\hline $\begin{array}{l}\text { Kelheim, tombe de } 1863 \\
\text { (Lkr. Kelheim, D) }\end{array}$ & 46,6 & 12,5 & 6,6 & 40 & $\begin{array}{c}3,2 \\
\text { flamme large }\end{array}$ & $\begin{array}{c}6,1 \\
\text { douille courte }\end{array}$ & épée & LIDI & $\begin{array}{l}\text { Krämer 1985: } \\
137 \text { pl. } 70 \mathrm{~A} 2 \text {. }\end{array}$ \\
\hline $\begin{array}{l}\text { Verna, tombe de } 1818 \\
\text { (Dép. Isère, F) }\end{array}$ & 49,3 & ? & 7,1 & 42,2 & ? & 5,9 & ? & Lt DI & \\
\hline $\begin{array}{l}\text { Nierstein, tombe } \\
\text { (Kr. Mainz-Bingen, D) }\end{array}$ & 51 & 8,4 & 7,8 & 43,2 & 5,1 & 5,5 & épée, bouclier & LIDI & $\begin{array}{l}\text { Behrens } 1923 \text { : } \\
22 \text { fig. } 27 \text {. }\end{array}$ \\
\hline $\begin{array}{l}\text { Wederath, tombe } 463 \\
\text { (Kr. Bernkastel-Wittlich, D) }\end{array}$ & 56,2 & 11,2 & 8,9 & 47,3 & 4,2 & 5,3 & - & Lt Dla & $\begin{array}{l}\text { Haffner 1974b, } \\
\text { pl. } 144,12 .\end{array}$ \\
\hline $\begin{array}{l}\text { Nîmes "Grézan", } \\
\text { tombe (Dép. Gard, F) }\end{array}$ & 63,2 & ? & 8 & 55,2 & ? & \begin{tabular}{c|}
6,9 \\
douille courte
\end{tabular} & ? & $\mathrm{LIClD}$ & $\begin{array}{l}\text { Py 1981: } \\
191 \text { fig. } 87 .\end{array}$ \\
\hline $\begin{array}{l}\text { Trier/Olewig, tombe } \\
\text { (Kr. Trier-Saarburg, D) }\end{array}$ & 67,5 & $?$ & 8,5 & 59 & $?$ & \begin{tabular}{c|}
6,9 \\
douille courte
\end{tabular} & \begin{tabular}{|l|} 
épéc, casque, \\
2' lance
\end{tabular} & Lt D2a & $\begin{array}{l}\text { Schindler } 1971 \text { : } \\
50 \text { fig. } 7.9 \text {. }\end{array}$ \\
\hline
\end{tabular}

Tableau 10 -Choix de grandes pointes de lances en forme de feuille de laurier dans des tombes de La Tène $C$ et $D$ en France et en Allemagne. Les fers de lances en forme de feuille de saule, les lances à lames ondulées et les autres formes de pointes de lance ne sont pas inclus.

La lame, dans le fourreau, à nervure centrale et à deux cannelures droites, est divisée en cinq parties, les deux tranchants plus épais n'ayant pas été rajoutés par le forgeron $^{113}$. Le passage entre la lame et la soie à section rectangulaire se fait par un épaulement. 
Il existe encore une quatrième épée $\left(\mathrm{n}^{\circ} 4\right)$ avec une lame plus mince et exceptionnellement plus effilée que les autres (long. $90 \mathrm{~cm}$, larg. 3,9-3,5 cm). Elle est pliée au milieu, formant un angle d'environ $60^{\circ}$ (fig. 43 a). Sa section est ovale et ses tranchants sont parallèles avec une pointe courte. La lame se raccorde par un angle droit à la soie à section rectangulaire, avec un rivet terminal.

Il existe également des fragments de fourreau en tôle de fer dont la largeur (larg. 3,9 $\mathrm{cm}$ ) correspond à celle de cette lame (fig. 43 b).

Une autre épée $\left(n^{\circ} 5\right)$ est représentée par un unique petit fragment de sa lame (long. $10,2 \mathrm{~cm}$, larg. 4,6 cm), dont les bords indiquent qu'elle était aussi pliée (fig. 44). Peutêtre correspond-elle aux restes d'un fourreau en bronze plié (larg. 4,7/5,3 cm). Toutefois, d'après son état de conservation, il est probable que ces fragments de fourreau appartiennent plus à cette épée qu'à l'épée entière non pliée $\left(n^{\circ} 2\right)$.

\section{LES POINTES ET LE TALON DE LANCE}

Le matériel comprend aussi six pointes de lances et un petit talon de lance (fig. 38, 45, 46) en forme de petite douille (long. $4,1 \mathrm{~cm}$, dia interne de la douille 1,5 cm). Cinq de ces flammes de lances sont en forme de feuille de laurier, un type que l'on distingue de celles à flamme symétrique et en forme de feuille de saule. Elles portent toutes les traces du bûcher et, sont très bien conservées. Trois de ces six fers de lances sont figurés sur les anciennes planches (fig. 4, objet DD ; fig. 5, objets IV et VII) qui représentent les trois types présents à Verna; ils sont probablement évoqués par le term "plusieurs armes" de la documentation déjà mentionnée.

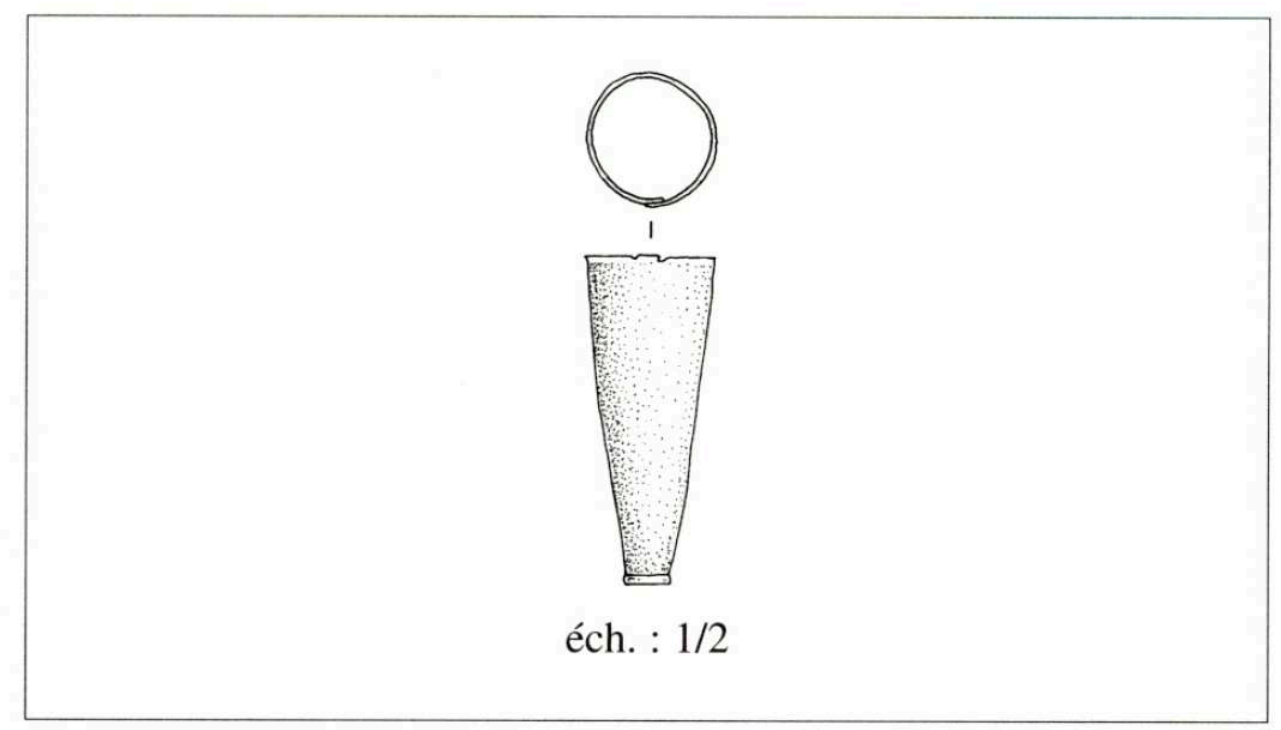

45-Talon de lance (fer)

Une pointe de lance exceptionnellement grande $\left(\mathrm{n}^{\circ} 1\right)$, d'une longueur de $48,5 \mathrm{~cm}$, comporte une flamme en forme de feuille de laurier à nervure médiane (fig. 46a). La largeur des ailerons n'est pas déterminable avec précision à cause de la corrosion. Sa douille de $7,2 \mathrm{~cm}$ de longueur et de $1,9 \mathrm{~cm}$ de diamètre interne est très petite comparée à la grande flamme puisqu'elle possède la même taille que les douilles des lances plus petites (cf. infra). 
Sa forme et sa taille sont typiques des lances qui se trouvent, pour la plupart, dans les tombes de La Tène C2 et D1 (tab. 10) (Schumacher 1999: 35-37). Les rapports entre longueur et largeur de la flamme, et longueur de la flamme et de la douille peuvent être retenus comme critères typométriques. Les minima et maxima de ces données relatives permettent de leur appliquer les termes "flamme mince" ou "large" et "douille courte" ou "longue".

Les lances avec des pointes de cette taille sont limitées dans leur utilisation réelle par la taille de leur douille. Lorsque les douilles sont trop petites pour laisser exercer une pression sur la pointe, il faut plutôt envisager une fonction d'insignes. Par contre, elles ne se rencontrent pas uniquement dans les tombes des élites militaires caractérisées par d'autres équipements. Il est remarquable que les grandes et larges pointes de lances sont moins nombreuses dans les tombes de La Tène D2 que dans celles des phases précédentes $^{114}$. A cette époque, les épées sont souvent les seuls représentants d'une panoplie d'armes dans les tombes et les lances de dimensions extraordinaires jouent un rôle moins important dans les rites funéraires. Des tombes des membres de l'élite militaire, comme celles de Goeblingen-Nospelt (Thill 1967 : 97-98, pl. 3, 11-14), sont tout de même équipées avec des lances, même si, avec une longueur de $20 \mathrm{~cm}$, elles sont moins impressionnantes.
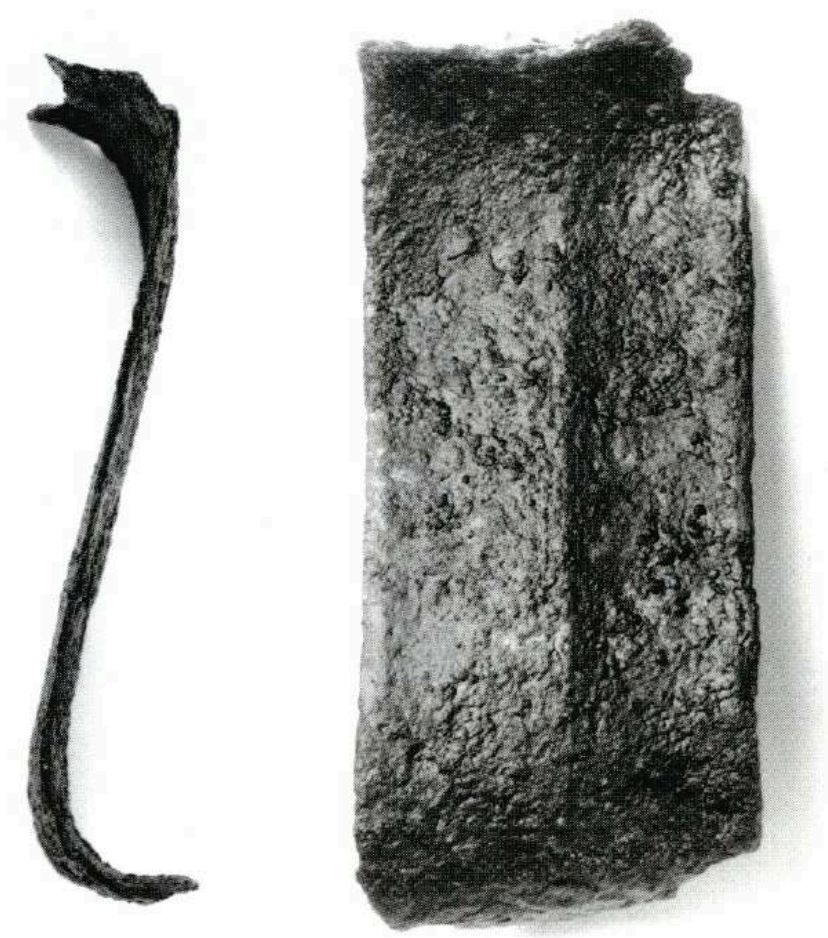

44 - Fragment de l'épée, $n^{\circ} 5$ 


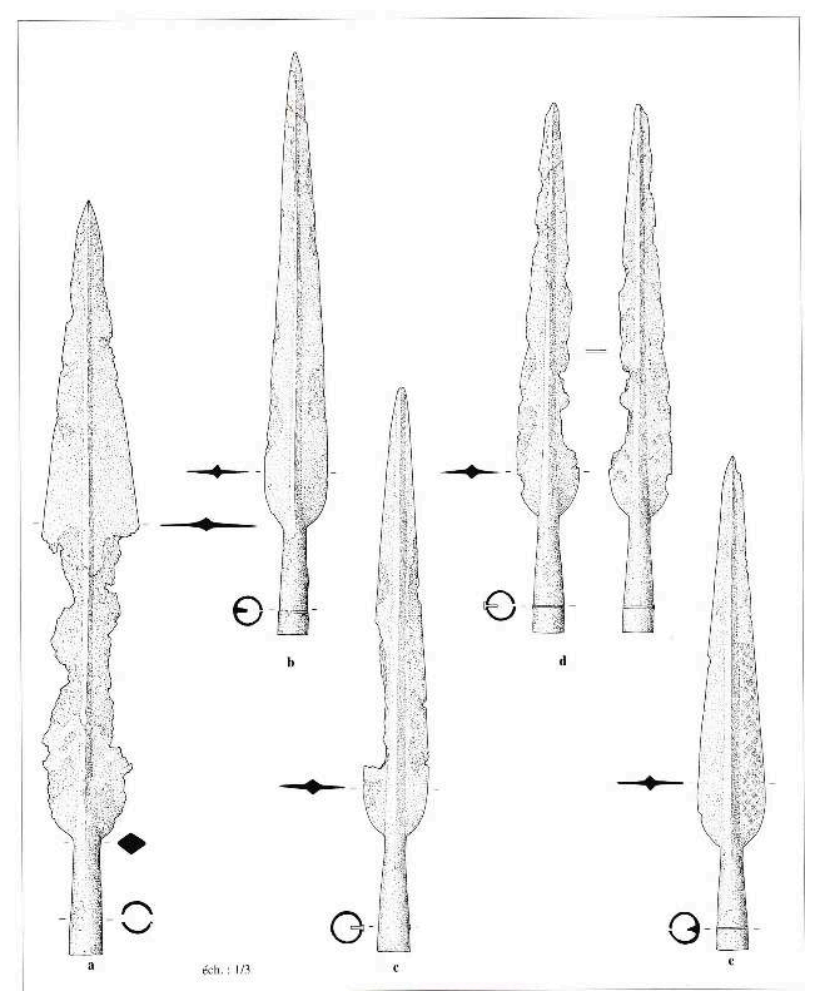

46- Pointes de lances

Il y a plusieurs explications à ce phénomène : l'importance de la lance comme insigne de puissance du guerrier dans le rituel funéraire et/ou dans la vie quotidienne a diminué. Peut-être ce processus est-il aussi en relation avec la cavalerie, qui gagne de l'importance à La Tène D2, et qui a besoin d'autres armes que d'une lance avec de grands ailerons.

Dans ce contexte, il faut peut-être associer cette grande lance avec le troisième mors de la tombe, qui a pu appartenir à un équipement de cavalier. Dans une tombe exceptionnelle, la présentation du statut de cavalier et de guerrier à pied, chacun avec ses insignes funéraires, est possible.

Dans les régions celtiques de l'Est, les pointes de lances très longues reflètent des traditions hallstattiennes ${ }^{115}$ et sont également assez nombreuses dans les tombes de La Tène $\mathrm{C}^{116}$.

137 Les autres lances ( $\left.n^{\circ} 2-5\right)$, à flammes en forme de laurier et à nervure médiane, ont des tailles plus raisonnables (b:long. $37,6 \mathrm{~cm}$, larg. $3,9 \mathrm{~cm} ; \mathrm{c}$ : long. $36,2 \mathrm{~cm}$, larg. $4,1 \mathrm{~cm} ; \mathrm{d}$ : long. $34,0 \mathrm{~cm}$, larg. $3,9 \mathrm{~cm}$; e : long. $31,3 \mathrm{~cm}$, larg. $4,1 \mathrm{~cm}$ ) (fig. 46 b à e). Elles sont décorées d'une ligne qui entoure la douille. La restauration a permis de découvrir sur les ailerons de plusieurs exemplaires $\left(n^{\circ} 4,5\right)$ des décors très fins constitués d'un filet de petits rhombes (fig. 47). Les impressions peu profondes laissent penser à un travail à l'acide plutôt qu'au poinçon : la surface était recouverte par la cire, puis le décor incisé était traitée par l'acide. Ce type de décors est rare en Gaule, mais fréquent dans les régions orientales de la culture laténienne ${ }^{117}$; leur répartition a généralement été liée aux mouvements de populations "germaniques" par la recherche germano-centriste au début du XXe s. (Kossinna 1905 ; Jahn 1913). Cependant la culture de La Tène utilise elle aussi cette technique dont les rares exemples sont toujours le fait d'une conservation extraordinaire $^{118}$. 


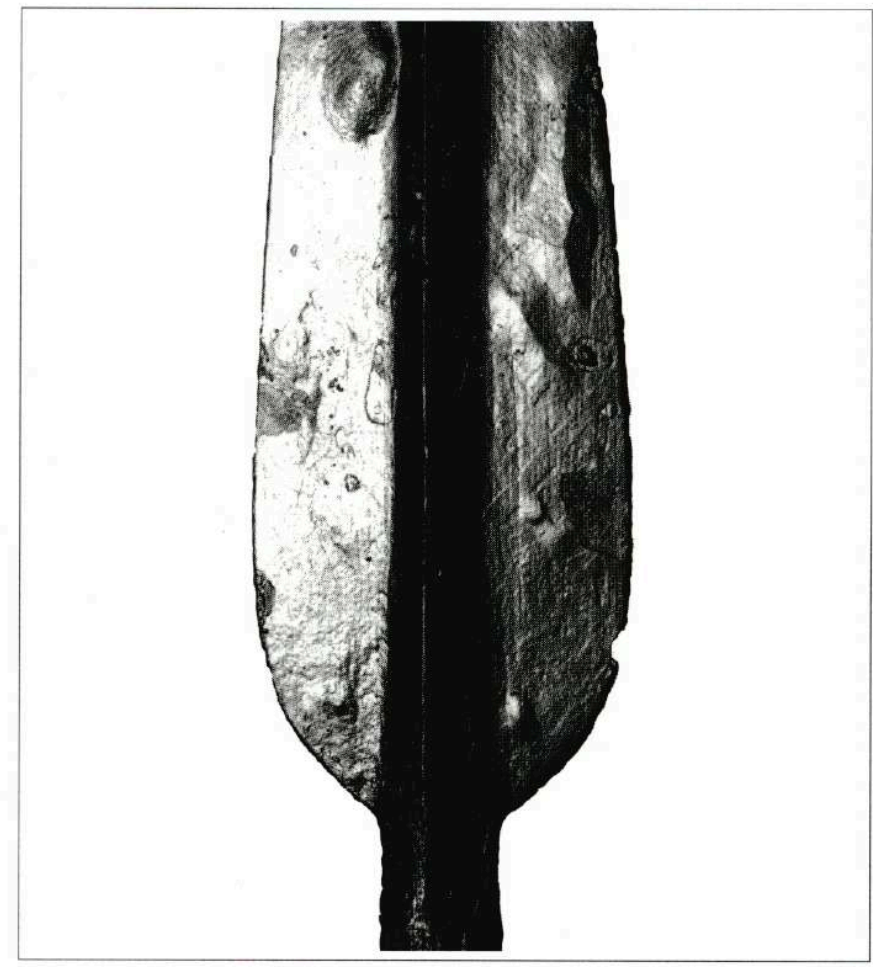

47 - Décor sur un aileron de la pointe d'une lance

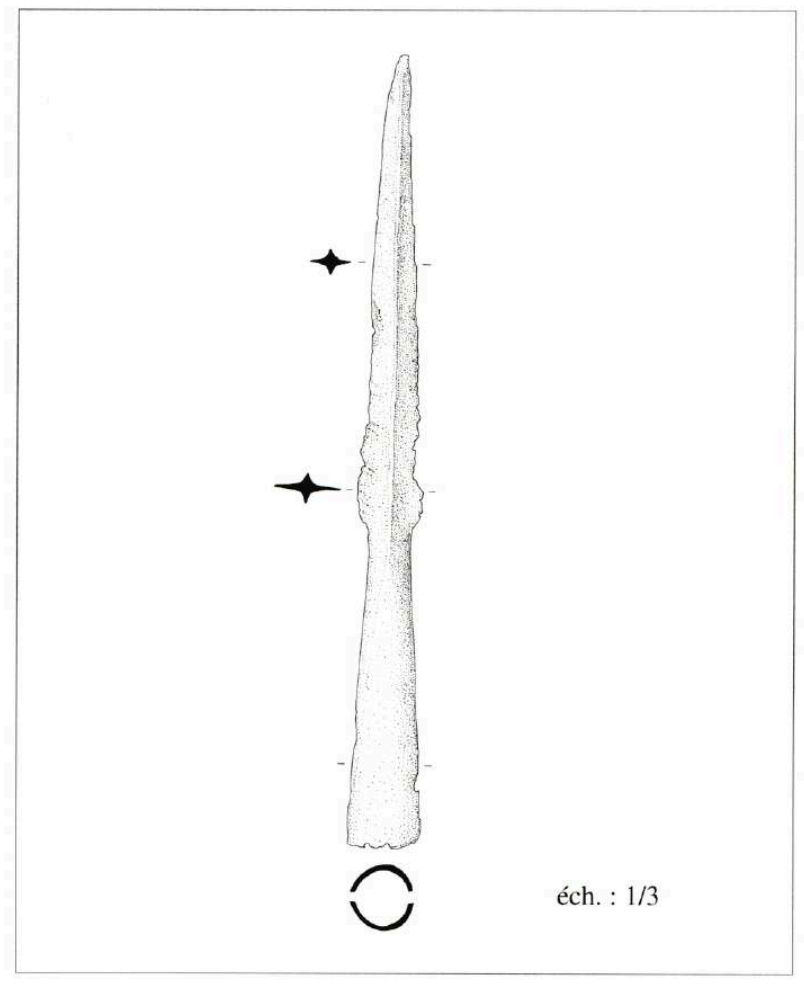

48 - Fer de lance très effilé, $n^{\circ} 6$

Quant à leur morphologie, les lances décorées de Verna sont clairement de production celtique; le seul décor dit "germanique" ne suffit donc pas à prouver une origine de l'Est de l'Europe centrale comme c'est probablement le cas pour des pointes de lances 
décorées de lignes en zigzag vertical, de forme plus effilée et à nervure forte, qui témoigneraient de contacts à longue distance. Ainsi, les pièces trouvées à Port (canton de Bern, $\mathrm{CH}$ ) ou à Bludenz (Vorarlberg, A) ${ }^{119}$ correspondent en tous points aux exemplaires de Pologne ${ }^{120}$. Les objets de Verna, comme ceux de Walduelversheim (Kr. Mainz-Bingen, D) et de Kelheim (Lkr. Kelheim. D) semblent plus être des produits locaux ${ }^{121}$.

La mise en évidence de ce type de décor sur les lances de Verna résulte de la conservation par le feu, de leur sauvegarde dans une collection aux XIXe et XXe s. et enfin d'une restauration moderne. Dès lors, il est possible d'envisager d'autres découvertes de tels décors sur armes.

Enfin, un fer de lance ( $n^{\circ}$ 6) diffère totalement des autres pointes de Verna (fig. 48), très effilé, à forte nervure centrale (long. $24,9 \mathrm{~cm}$, larg. $2,1 \mathrm{~cm}$ ) et douille relativement longue et forte (dia. $2,3 \mathrm{~cm}$, long. $10 \mathrm{~cm}$ ). Cet exemplaire avec sa douille puissante et sa flamme en forme de baïonnette correspond plus que les autres lances à une arme de guerre. Ainsi, S. Sievers a, pour les armes comparables d'Alésia, privilégié l'interprétation guerrière à celle d'objets de prestige ${ }^{122}$.

\section{LES UMBOS DE BOUCLIER}

141 Dans le mobilier de Verna, on distingue trois umbos à ailettes rectangulaires qui ne diffèrent que par quelques détails. Tous ont en commun des ailettes courtes et une coque sphérique à rebords à cornière. Des critères discriminatoires fins et fréquents permettent de proposer une typologie.

Les trois umbos et leurs clous ont été arrachés de leurs boucliers puis brûlés ; de plus, suite à la fouille de 1818, on a tenté de déplier quelques-unes des ailettes.

Le premier umbo $\left(\mathrm{n}^{\circ} 1\right)$ a des ailettes étroites et quasi rectangulaires (fig. 49 a) avec deux trous (horizontaux) qui contiennent des clous courts (tiges courbées et petites têtes rondes). Ce type d'umbo, dénommé type de Nierstein d'après une tombe centrale de l'aire de répartition (tab. 11), est daté de La Tène D1 si l'on se réfère aux ensembles clos. Il se distingue des autres umbos de La Tène $\mathrm{C}$, à ailettes plus larges et coques plus hautes ${ }^{123}$.

Les clous intérieurs de cet umbo se distingue par des têtes creuses avec des bords en bourrelet de $1,1 \mathrm{~cm}$ de diamètre, à l'origine recouverts d'émail dégradé par le feu du bûcher. Des clous émaillés, plus grands, sont caractéristiques des umbos de La Tène $\mathrm{C}^{124}$, mais inhabituels pour La Tène $\mathrm{D}$, les décors striés étant plus courants pour l'émail. Toutefois la petite taille des clous correspond bien à ceux de ce type d'umbo.

Aucun manipule n'a été trouvé à Verna, bien que parfois des exemplaires à bande en fer à bouts recourbés aient été conservés (tab. 11) comme par exemple dans les tombes 1178 et 1216 de Wederath (Kr. Bernkastel-Wittlich, D), dans la tombe I.12 de "La Noue Mauroy" d'Acy-Romance (dép. Ardennes) ou l'un des sept exemplaires de la Viereckschanze de Nordheim-Bruchhöhe (Kr. Heilbronn, D). Ces manipules sont caractéristiques de ce type d'umbo. Dans la tombe I.101 d'Acy-Romance "La Croizette" un manipule de ce type et un crochet de ceinture apparaissent comme les uniques traces de la panoplie de guerrier (Lambot, Friboulet, Méniel 1994 : 30-33 fig. 21, 17, 19). 
corrosion importante, une restauration insuffisante ou une forte utilisation de résines artificielles peuvent cacher les trous des clous et une confusion des types est alors possible ${ }^{126}$.

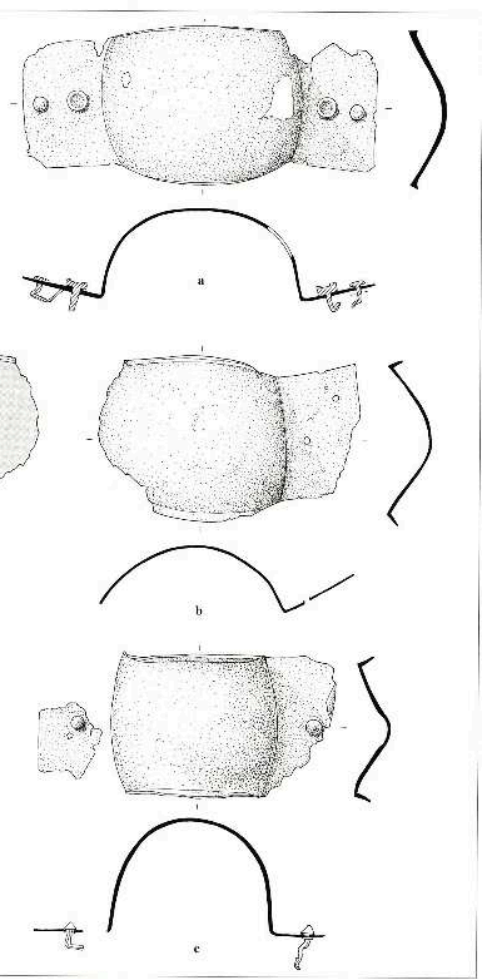

\section{A - UMBO N 1 ; UMBO N 2 (FER BRONZÉ) ; UMBO N 3}

\begin{tabular}{|c|c|c|}
\hline Lieu de découverte & Circonstances & Bibliographie \\
\hline $\begin{array}{l}\text { Acy-Romance « La Noue Mauroy » } \\
\text { (Dép. Ardennes) }\end{array}$ & Umbo avec manipule ; tombe à épée, couteau & Lejars 1996 : 84 fig. 3B. \\
\hline $\begin{array}{l}\text { Gournay } \\
\text { (Dép. Oise) }\end{array}$ & $\begin{array}{l}\text { Sanctuaire avec dépositions d' armes, } \\
1 \text { fragment d'umbo avec manipule ( } 3607)\end{array}$ & $\begin{array}{l}\text { Brunaux / Rapin 1988, pl. } 12 \text { (1145), } \\
14 \text { (1396), } 36 \text { (3607). }\end{array}$ \\
\hline Montmartin (Dép. Oise) & Déposition rituelle dans un habitat & Lejars in : Brunaux/Méniel 1997 : 158 fig. 119, 4. \\
\hline Verna (Dép. Isère) & Tombe de 1818 & \\
\hline Thayngen (Kt. Schaffhausen, CH) & Trouvé dans une grotte, incinération? & Tanner 1979: $76 \mathrm{pl} .23$. \\
\hline $\begin{array}{l}\text { Oleggio, tombe } 53 \\
\text { (Prov. Novara, I) }\end{array}$ & $\begin{array}{l}\text { Tombe avec épée, lance, couteau à découper, } \\
\text { broche à rôtir, ciseaux, céramiques }\end{array}$ & Spagnolo Garzoli 1999 : 114 fig. $95,3$. \\
\hline $\begin{array}{l}\text { Manching } \\
\text { (Lkr. Pfaffenhofen a. d. Ilm, D) }\end{array}$ & Oppidum (trouvaille isolée) & Musée d'Ingolstadt. \\
\hline Nierstein (Kr. Mainz-Bingen, D) & Incinération avec épée, lance, couteau à découper & Behrens $1927: 62$, Nr. 223,3 . \\
\hline $\begin{array}{l}\text { Nordheim « Bruchhöhe " } \\
\text { (Kr. Heilbronn, D) }\end{array}$ & $\begin{array}{l}\text { Déposition rituelle de } 8 \text { umbos, un avec manipule, } \\
\text { dans la Viereckschanze }\end{array}$ & Neth $2000: 77$. \\
\hline $\begin{array}{l}\text { Oderberg, tombe } 1 \\
\text { (Kr. Eberswalde, D) }\end{array}$ & $\begin{array}{l}\text { Incinération avec épée courte, pointe et talon de } \\
\text { lance, couteau (?), vase en céramique }\end{array}$ & Seyer 1982, pl. 18, 2. \\
\hline $\begin{array}{l}\text { Offenbach « Bürgel » } \\
\text { (Stadt Offenbach, D) }\end{array}$ & $\begin{array}{l}\text { Incinération avec épée, lance, ciseau, rasoir, fibule, } \\
\text { vaisselles céramiques }\end{array}$ & Nahrgang $1967: 167$ fig. $166,12$. \\
\hline $\begin{array}{l}\text { Plaidt «In der Kreuzchen » } \\
\text { (Kr. Mayen-Koblenz, D) }\end{array}$ & Tombes à incinération & $\begin{array}{l}\text { Oesterwind } 1989: 108 \text { sq. fig. } 28,1 \text {; } \\
\text { Germania } 15,1931: 110 .\end{array}$ \\
\hline Schwangau (Lkr. Ostallgäu, D) & Sanctuaire de type alpin & Maier $1985: 237$ fig. 5,5 . \\
\hline $\begin{array}{l}\text { Wallau } \\
\text { (Rheingau-Taunus-Kreis, D) }\end{array}$ & $\begin{array}{l}\text { Incinération sous un tumulus, avec épée, lance, } \\
\text { ciseau, fibule, anneau en fer, céramique }\end{array}$ & Schoppa 1961 : 169 fig. 8,5 . \\
\hline $\begin{array}{l}\text { Wederath, tombe } 1178 \\
\text { (Kr. Bernkastel-Wittlich, D) }\end{array}$ & $\begin{array}{l}\text { Umbo avec manipule ; incinération avec épée, } \\
\text { pointe et talon de lance, couteau à découper, hache, } \\
\text { balance de précision, vaisselles céramiques }\end{array}$ & Haffner 1978, pl. 297, 1. 9. \\
\hline $\begin{array}{l}\text { Wederath, tombe } 1216 \\
\text { (Kr. Bernkastel-Wittlich, D) }\end{array}$ & $\begin{array}{l}\text { Umbo avec manipule ; incinération double, homme } \\
\text { avec épée, lance, rasoir }\end{array}$ & Haffner 1978, pl. 309, 5;310, 8. \\
\hline
\end{tabular}

Tableau 11 -Liste des umbos à ailettes de type Nierstein 


\begin{tabular}{|l|l|l|}
\hline \multicolumn{1}{|c|}{ Lieu de découverte } & \multicolumn{1}{|c|}{ Circonstances } & \multicolumn{1}{c|}{ Bibliographie } \\
\hline $\begin{array}{l}\text { Hières-sur-Amby « Larina » } \\
\text { (Dép. Isère, F) }\end{array}$ & $\begin{array}{l}\text { Oppidum / dépôt (troisième trou de rivet vu sur } \\
\text { l'original à la Maison du Patrimoine de } \\
\text { Hières-sur-Amby) } \\
\text { Tombe avec épée, lance, céramique }\end{array}$ & Perrin $1990: 95$ fig. 100. \\
\hline $\begin{array}{l}\text { Nîmes « Camplanier » } \\
\text { tombe 1 (Dép. Gard, F) }\end{array}$ & Tombe avec pointe de lance, boite, paire de strigiles & Feugère 1996: 179 fig. 15, 12. \\
\hline $\begin{array}{l}\text { Nîmes « Mail romain », tombe 225 } \\
\text { (Dép. Gard, F) }\end{array}$ & Tombe de 1818 & Debord 1996: 72 fig. 31, 2. \\
\hline Verna (Dép. Isère, F) & Oppidum & Tizzoni 1982, pl. 38 g. \\
\hline $\begin{array}{l}\text { Villeneuve-Saint-Germain } \\
\text { (Dép. Aisne, F) }\end{array}$ & Tombes & \\
\hline Barzio 1905 (Prov. Como, I) & & \\
\hline
\end{tabular}

Tableau 12 - Liste des umbos à ailettes de type Nîmes

Un autre umbo ( $\left.\mathrm{n}^{\circ} 2\right)$ est à ailettes courtes trapézoïdales (fig. 49 b), avec trois trous pour les clous dans les coins externes et au milieu des ailettes. La coque caractéristique des formes tardives est sphérique avec des bords à cornière. Cette forme de La Tène D1 peut être appelée type de Nîmes (tab. 12).

La surface de l'exemplaire de Verna est recouverte à l'intérieur et à l'extérieur d'une fine couche de bronze, appliquée selon une technique bien connue; une poudre de bronze est brasée directement sur le fer à l'aide de cire, ce qui empêche une oxydation anticipée (Ankner, Hummel 1985 : 196-206). Ce traitement des objets en fer n'est pas très fréquent ( $c f$. infra B. Herbold). Il est connu par exemple pour trois "Hollow-piece rings" en fer de Écury-le-Repos "Le Crayon" (dép. Marne) de La Tène B2 ${ }^{127}$ Pour La Tène $\mathrm{C}$, on connaît deux mors bronzés d'un filet à canons articulés, un sur le site même de La Tène (canton de Neuchâtel, $\mathrm{CH}$ ), l'autre dans le dépôt de Lozna (Jud. Botosani, RO) ${ }^{128}$. De plus, sur l'oppidum de Manching (Lkr. Pfaffenhofen a. d. Ilm, D) a été identifié un fragment de lame d'épée entièrement recouverte par du bronze ${ }^{129}$. Enfin, sur le fourreau de la tombe 1178 de Wederath, des feuilles de bronze ont été soudées à l'étain sur le fer. Avec cette dernière technique, la surface n'est alors plus entièrement recouverte, seul un ornement étant appliqué sur certaines parties du fourreau ${ }^{130}$.

Les umbos en bronze demeurent très rares en Europe pour cette époque, alors que durant l'Empire romain les umbos ronds en bronze deviennent plus fréquents. Pour l'époque celtique, il est douteux qu'un umbo en bronze corresponde à l'idéal d'un umbo fastueux, plus prestigieux ou plus fonctionnel. L'utilisation de bronze à Verna pourrait l'avoir été dans un but décoratif, mais aussi pratique : l'oxydation des objets en fer pose de réels problèmes, alors qu'un traitement avec du bronze prévient une oxydation grave.

Pourquoi le choix de ce procédé pour l'umbo de Verna? Grâce aux moyens de restaurations modernes, il sera désormais possible de retrouver les traces de cette technique; en effet les traces de cuivre restent habituellement masquées par les produits d'oxydation du fer.

Le troisième umbo $\left(\mathrm{n}^{\circ} 3\right)$ quant à lui, conserve une seule ailette détériorée (fig. 49 c). Un petit clou à tête triangulaire est placé au centre de l'ailette; l'umbo ayant été démonté, le clou a perdu sa forme recourbée d'origine. De plus, les angles et les bords de l'ailette étant détériorés, il est impossible d'identifier un type précis; toutefois la petite taille du clou laisse penser à un groupement à trois, avec d'autres exemplaires dans les coins de l'ailette.

La forme de la coque n'est pas aussi bombée que sur les autres exemplaires, elle a un aspect plus cylindrique. 
153 Avec les exemplaires de Verna, s'ouvre la possibilité de décrire les formes les plus importantes des umbos à ailettes rectangulaires de La Tène D1. Le type de Nierstein comme celui de Nîmes ont des antécédents dans des formes de La Tène $\mathrm{C}^{131}$. Pour la phase D1, on peut ajouter d'autres formes, comme celle trouvée à Sion avec un seul grand clou dans chaque ailette ${ }^{132}$ ainsi que la forme de l'umbo de la tombe 1228 de Wederath (Kr. Bernkastel-Wittlich, D) avec des ailettes courbées vers le bas (Haffner 1978, pl. 314, 3), qui reste encore sans comparaison. Une autre forme à deux clous placés verticalement l'un en dessous de l'autre a été trouvée à Nîmes (tombe de l'Octroi de Beaucaire, dép. Gard) (Py 1981: 146, fig. 64, 8) et dans la tombe 2000 de Wederath (Cordie-Hackenberg, Haffner 1997 : 34, pl. 543 d).

154 On trouve ensuite d'autres types d'umbos à ailettes rectangulaires, plutôt typiques de La Tène D2 (Oesterwind 1989 : 108 ; Haffner dir. 1989 : 71). Une forme spécifique, le type Lamadeleine, à ailettes triangulaires avec extrémités et coque presque rondes et des bords à cornière, commence déjà à La Tène $\mathrm{D} 1$, mais la plupart des ensembles datent de La Tène D2 (tab. 13). Leur concentration dans la région située entre la rivière Moselle et sa confluence avec le Rhin (fig. 50) est liée à la fréquence du rite funéraire avec armes et à sa continuité à La Tène D2 dans cette région, mais peut être aussi à une réelle concentration locale de ce type. Hormis ce type-là, on peut distinguer un autre type d'umbo tardif avec des ailettes hautes et trapézoïdales. On le connait à Alésia (dép. Côte-d'Or), à Ribemont-sur-Ancre (dép. Oise) et dans la tombe 27 de "La Catalane" des Baux-de-Provence (dép. Bouches-du-Rhone) ${ }^{133}$; avec une pointe sur la coque, à Soissons (dép. Aisne) et à Owslebury (Hampsshire, GB) ${ }^{134}$. Contrairement aux autres umbos à ailettes rectangulaires, les deux derniers sont en bronze. 


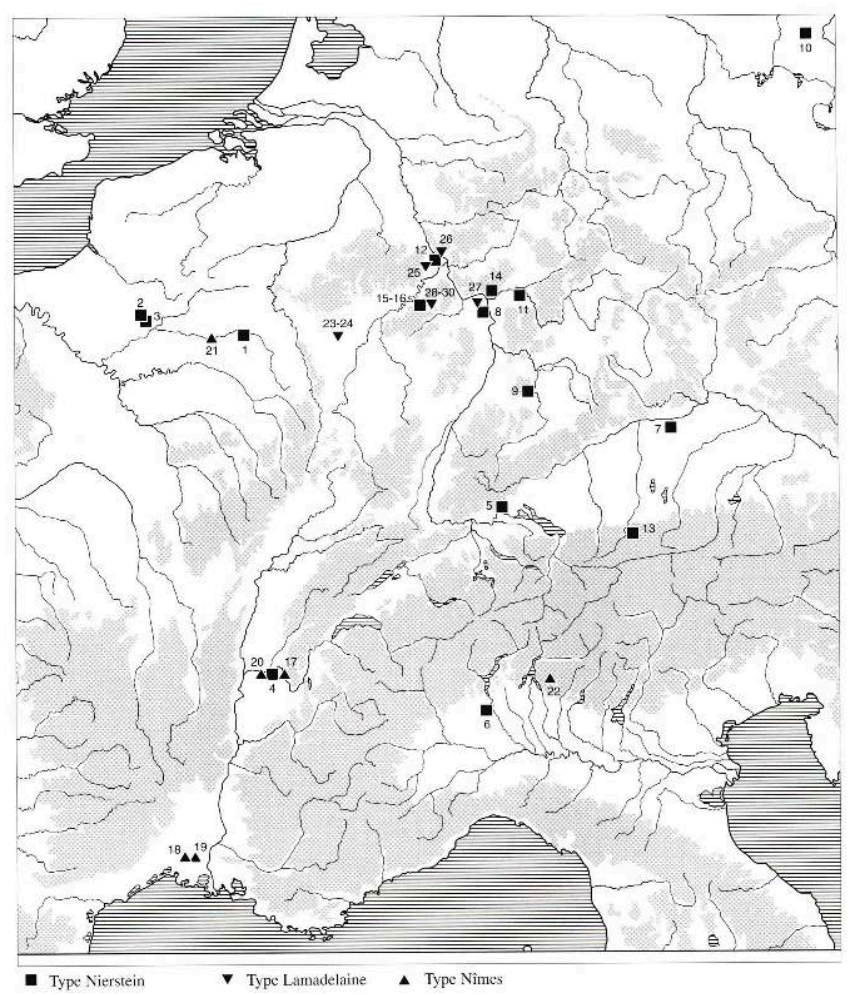

50-Répartition des umbos à ailettes de type Nierstein, Nîmes et Lamadelaine (voir tab. 11 à 13). Type Nierstein : 1 Acy-Romance/La Noue Mauroy. 2 Gournay. 3 Montmartin. 4 Verna. 5 Thayngen. 6 Oleggio, tombe 53. 7 Manching. 8. Nierstein. 9 Nordheim/Bruchhöhe. 10 Oderberg. tombe 1. 11 Offenbach am Main/Bürgel. 12 Plaidt/In der Kreuzchen. 13 Schwangau. 14 Wallau. 15-16 Wederath tombe 1178 et 1216. Type Nîmes : 17 Hières-sur-Amby/Larina. 18 Nîmes. 19 Nîmes/Mail romain, tombe 225. 20 Verna. 21 Villeneuve-Saint-Germain. 22 Barzio 1905. Type Lamadelaine : 23-24 Titelberg/Lamadelaine, tombe 3 et 59. 25 Ettringen/Sinberg, tombe 10. 26 Heimbach-Weis, tombe 2. 27 Ober-Olm. 28-30 Wederath tombe 90, 481 et 1726.

\begin{tabular}{|l|l|l|}
\hline \multicolumn{1}{|c|}{ Lieu de découverte } & \multicolumn{1}{|c|}{ Circonstances } & \multicolumn{1}{c|}{ Bibliographie } \\
\hline $\begin{array}{l}\text { Titelberg/Lamadelaine, } \\
\text { tombe 3 (L) }\end{array}$ & $\begin{array}{l}\text { Tombe, entre autres avec épée, } \\
\text { pointe de lance, mors (Lt D2a) }\end{array}$ & Metzler-Zens et al. 1999: 33 fig. 19,2. \\
\hline $\begin{array}{l}\text { Titelberg/Lamadelaine, } \\
\text { tombe 59 (L) }\end{array}$ & Tombe avec une clé de boite (Lt D2) & Metzler-Zens et al. 1999: 208 fig. 222, 1. \\
\hline $\begin{array}{l}\text { Ettringen « Sinberg », tombe 10 } \\
\text { (Kr. Mayen-Koblenz, D) }\end{array}$ & $\begin{array}{l}\text { Tombe avec pointe de lance, } \\
2 \text { « geknickte Fibeln », } \\
\text { 2 vaisselles en terre cuite (Lt D1) }\end{array}$ & Oesterwind 1989, pl. 14 C 2. \\
\hline $\begin{array}{l}\text { Heimbach-Weis, tombe 2 } \\
\text { (Kr. Neuwied, D) }\end{array}$ & $\begin{array}{l}\text { Tombe avec char, ustensiles de cuisine, équipement } \\
\text { de cavalier, armes, entre autres un deuxième } \\
\text { umbo rond (Lt D2a) }\end{array}$ & Joachim 1973. \\
\hline Ober-Olm (Kr. Mainz-Bingen, D) & Cimetière & Behrens 1923:31 fig. 36. \\
\hline $\begin{array}{l}\text { Wederath, tombe 1726 } \\
\text { (Kr. Bernkastel-Wittlich, D) }\end{array}$ & $\begin{array}{l}\text { Tombe avec épée, pointe de lance, « geknickte } \\
\text { Fibel » vaisselles en terre cuite (Lt D2a) }\end{array}$ & $\begin{array}{l}\text { Haffner (dir.) 1989: 71; } \\
\text { Thoma 1993, Form 7 Var. A. }\end{array}$ \\
\hline $\begin{array}{l}\text { Wederath, tombe 481 } \\
\text { (Kr. Bernkastel-Wittlich, D) }\end{array}$ & $\begin{array}{l}\text { Tombe avec épée, pointe de lance, vaisselles en } \\
\text { terre cuite (Lt D2a) }\end{array}$ & Haffner 1974b, pl. 148, 7. \\
\hline $\begin{array}{l}\text { Wederath, tombe 90 } \\
\text { (Kr. Bernkastel-Wittlich, D) }\end{array}$ & $\begin{array}{l}\text { Tombe avec épée, pointe de lance, couteau à } \\
\text { découper, 2 fibules, vaisselles en terre cuite (Lt D2a) }\end{array}$ & Haffner 1971, pl. 20, 6. \\
\hline
\end{tabular}

Tableau 13 -Liste des umbos à ailettes de type Lamadelaine

A La Tène D1, les premiers umbos ronds apparaissent dans le domaine celtique oriental et dans la région des Trévires (Schumacher 1999: 42 ; Luczkiewicz 1998). Que se passe$\mathrm{t}$-il pour la forme entière du bouclier? Des boucliers ronds sont plus adaptés à la cavalerie, mieux attestée pour La Tène $\mathrm{D}$ qu'auparavant ${ }^{135}$. Mais des représentations comme celles du chaudron de Gundestrup ou des arcs de triomphe augustéens de Provence, montrent aussi des boucliers allongés et angulaires avec des umbos ronds 
(Hachmann 1990 : 760-766 ; Amy et al. 1962 : 109, pl. 46, 54, 59, 48). Les connaissances sur la question restent très limitées à cause du rite de l'incinération et du faible nombre d'éléments en bois. A Verna, il manque, comme dans beaucoup d'autres tombes de La Tène $\mathrm{D}$, les gouttières des boucliers qui pourraient apporter plus d'informations. A La Tène $\mathrm{D}$, les boucliers déposés dans les tombes deviennent plus rares que pendant La Tène $C$, qui a livré un nombre important de guerriers avec des panoplies entières.

\section{LA COUVERTURE EN ALLIAGE CUIVREUX DE L'UMBO N². 2 . HerboldETAT DE L'OBJET}

L'umbo a été restauré dans les laboratoires du Römisch-Germanisches Zentralmuseum durant l'été 2000 (fig. 51). L'état de l'objet laissait prévoir une surface couverte de magnétite formée lors du passage au feu. Toutefois, la surface présentait quelques tâches rouge éclatant, qui ont été identifiées comme de l'hématite. Ceci est le résultat d'un procédé thermique visant à faire rougir les objets en milieu oxydant. Dans le cas présent, l'hématite se trouvait sur la surface en magnétite de l'objet, partiellement couverte de crasses.

157 A l'intérieur de l'umbo, une zone couverte de produits de corrosion au cuivre a été remarquée et d'abord interprétée comme les restes d'un objet inconnu en alliage cuivreux. Fondu lors de l'incinération antique, cet élément en alliage cuivreux aurait laissé des gouttes secondaires sur l'umbo. A l'aide d'une binoculaire, des traces ponctuelles de cet alliage cuivreux métallique de couleur or ont été discernées. Plusieurs petites zones avec des traces de corrosion de cuivre ont également été observées à l'extérieur de l'umbo.

\section{LA PRÉPARATION DE LA SURFACE ORIGINELLE}

L'état de l'objet a pu être observé grâce à des photos prises à fort grossissement. Ensuite, la surface originelle a été dégagée par la micro-sableuse (abrasif : corindon, 1,5 bar, 1-2 VT).

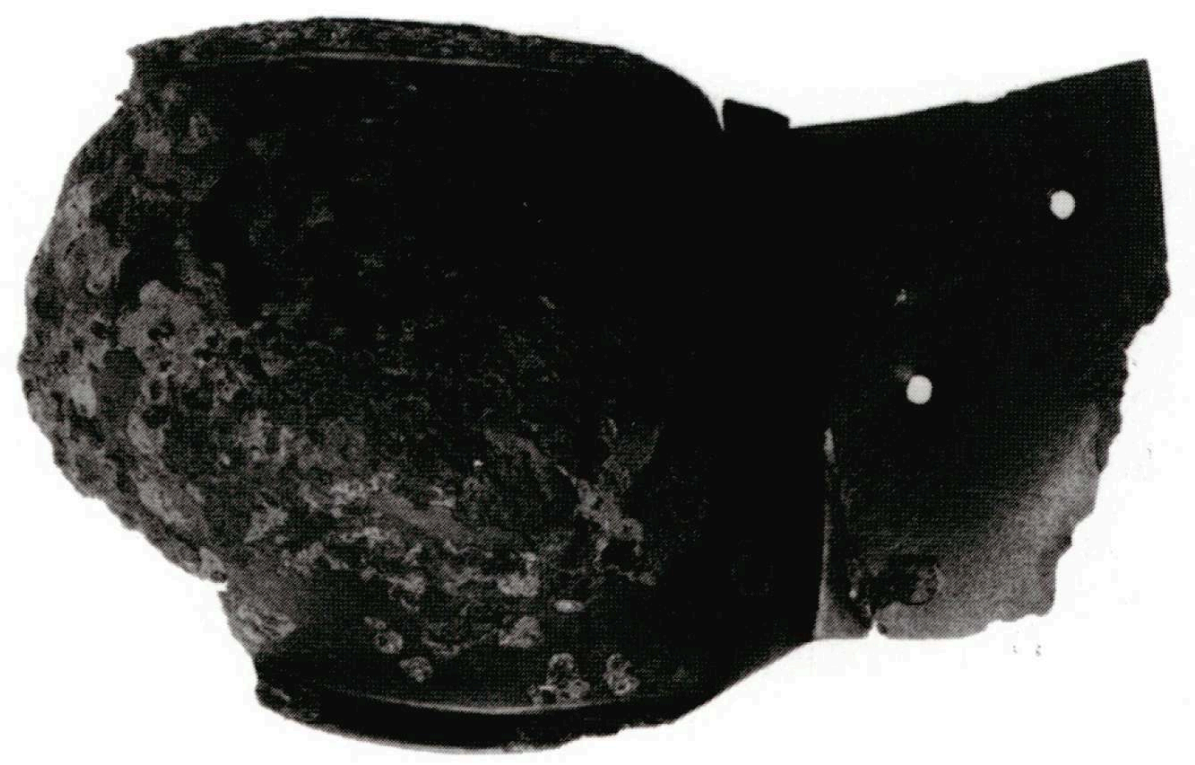

51-Umbo (fer bronzé) n² 
Le fragment d'ailette a une surface très stable, bien conservée par le feu, ce qui a facilité la détermination et la recherche de la surface originelle. L'intérieur de l'umbo a été sablé avec notamment un intérêt particulier pour les tâches de corrosion de cuivre. L'examen a commencé sur le côté de la cassure, vers le milieu du bombement. Si l'on s'attendait à des délimitations très précises des gouttes secondaires d'alliage cuivreux, en travaillant, la surface de couleur bronze s'est étendue plus que la corrosion visible au début.

Des discussions avec F. Hummel (RGZM) amènent à penser que cet umbo en fer était déjà couvert d'une pellicule d'alliage cuivreux durant l'Antiquité. Comme comparaison, on peut citer les cloches en fer romaines de moutons et de vaches, qui ont conservé une couverture en bronze comme protection contre la corrosion (Ankner, Hummel 1985 : 196-206). La technique qui consiste à recouvrir un objet en fer avec du cuivre ou un alliage cuivreux est bien connue: de la cire est mélangée avec une fine poudre de bronze ou de cuivre ; cette mixture est enduite sur la surface de fer polie et l'objet est chauffé dans un four.

Pour ce qui concerne l'umbo, la couche massive de magnétite se trouvant sur la couche d'alliage cuivreux semblait contredire cette interprétation. En fait, la magnétite a traversé la couche d'alliage cuivreux et s'est superposée pour donner l'image d'une couche originelle produite par l'incinération antique.

Pour vérifier cette hypothèse, des expérimentations ont été réalisées : une pièce en fer, couverte de cuivre sur la moitié, a été chauffée dans un four à $1000^{\circ} \mathrm{C}$ environ. La couverture de magnétite noire s'est formée sur la couche de cuivre, mais aussi sur la surface du fer non traitée. D'après $\mathrm{F}$. Hummel, ce phénomène est dû à la porosité de la fine couche de cuivre. Ces résultats s'appliquent à l'objet archéologique.

Il a été observé lors de la restauration de l'objet que l'umbo était entièrement recouvert par l'alliage cuivreux, sauf sur des petites zones très corrodées.

Nous avons décidé de laisser la couche en magnétite lorsque celle-ci était intacte ; par contre, là où elle avait éclaté, la couche d'alliage cuivreux a été dégagée avec la microsableuse (abrasif : microbilles de verre). La suite des couches (noyau en fer couverture en alliage cuivreux - couche de fer oxydé/magnétite) reste ainsi visible sur l'objet restauré.

\section{CONSERVATION FINALE}

Le fragment d'ailette a été collé au reste de l'umbo (colle : “Araldit, type $2020 \mathrm{~A} / \mathrm{B}$ ”) et la zone de cassure stabilisée par un tissu fin en verre à l'intérieur de l'umbo. A l'extérieur le joint a été réalisé par "Akemi Marmorkitt", et colorié avec une poudre de graphite pour l'harmonisation des couleurs. Finalement, l'umbo a été recouvert par un film de protection ("Paraloid B 72", dissout à l'acétone).

\section{CONCLUSION}

166 L'umbo de Verna montre que le traitement de l'objet en fer par bronzage était presque complètement masqué par une couche de magnétite. Ceci pose la question de la fréquence du bronzage, peut-être sous estimée, sur les objets de l'âge du Fer. Enfin, on peut s'interroger sur sa fonction : protection contre la corrosion ou décoration. 
167 La couche de magnétite, produite par le feu de l'incinération antique, est normalement définie comme la surface originelle de l'objet avec toutes ses informations, et aide à la protection et à la conservation. Cela incite le restaurateur à ne pas l'enlever. Dans notre cas, les tâches de corrosion de cuivre ont permis de découvrir la couverture en alliage cuivreux. Ces phénomènes seront à prendre avec plus de considération dans l'avenir.

\section{LE CHAR. M. Schönfelder}

168 L'étude du char de Verna suscite de nombreuses interrogations et discussions. Dès 1818, la présence d'un char à quatre roues était reconnue par l'identification de bandages de roues et d'autres éléments. A l'époque, c'est tout de même un char à deux roues qui a été restitué en dessin (fig. 52, pl. V), car d'autres types de char n'étaient pas envisageables. Cette reconstitution donne l'image d'un char de course antique, agrémenté de quelques éléments de Verna. En fait, il n'est plus possible de proposer une reconstitution entière car l'incinération a détruit trop d'informations.

Lors de la découverte, la présence du char a permis de dater la tombe de l'époque celtique. Cette datation correcte avant Oscar Montelius, Joseph Déchelette et tous les autres fondateurs de l'archéologie protohistorique est très étonnante et témoigne des bonnes connaissances archéologiques et historiques de Fr. Artaud.

Les chars à quatre roues de La Tène ne sont connus que par un petit nombre d'exemplaires ${ }^{136}$. L'interprétation de la fonction des éléments métalliques de ces chars est fondée sur des idées générales concernant les chars du Hallstatt jusqu'à ceux de l'époque moderne. Les analogies possibles entre les pièces de char de Verna avec d'autres chars restent rares et les parallèles sont très isolés géographiquement. Dès lors, les interprétations proposées pour la plupart des éléments techniques ne peuvent que l'être à titre d'hypothèses.

171 Quelques particularités, comme la tige de pivot en fer, sont probablement issues des méthodes méditerranéennes de construction des chars. Les chars romains de l'époque républicaine sont quasiment inconnus par l'archéologie. Seuls les chars des villes autour du Vésuve ${ }^{137}$ et quelques autres d'époque impériale restent la référence.

\section{LES ROUES}

172 Le rapport de fouille mentionne des bandages de roues en fer. Sur une planche (fig. 4, objet C.C.), y figure un fragment, probable exemple ; aucun clou n'est visible. A La Tène $\mathrm{C}$ et $\mathrm{D}$, les bandages de roues sont, en général, fixés sur les jantes d'une seule pièce en bois cintré (Schönfelder 2002) à la différence de La Tène A où des agrafes de jantes et des clous de fixations sont nécessaires. Ces bandages, encombrants, ne sont plus conservés; le diamètre, la largeur et l'épaisseur des roues de Verna restent donc inconnus.

Seules des frettes de moyeux en bronze sont conservées, entières ou sous la forme de fragments brûlés. De plus, quelques fragments ont été détruits volontairement après le bûcher - ainsi les frettes ont été pliées et déchirées. Étonnamment, de petits fragments de bronze, qui ne permettent aucune interprétation ni reconstitution, ont été collectés et gardés dans la collection. 


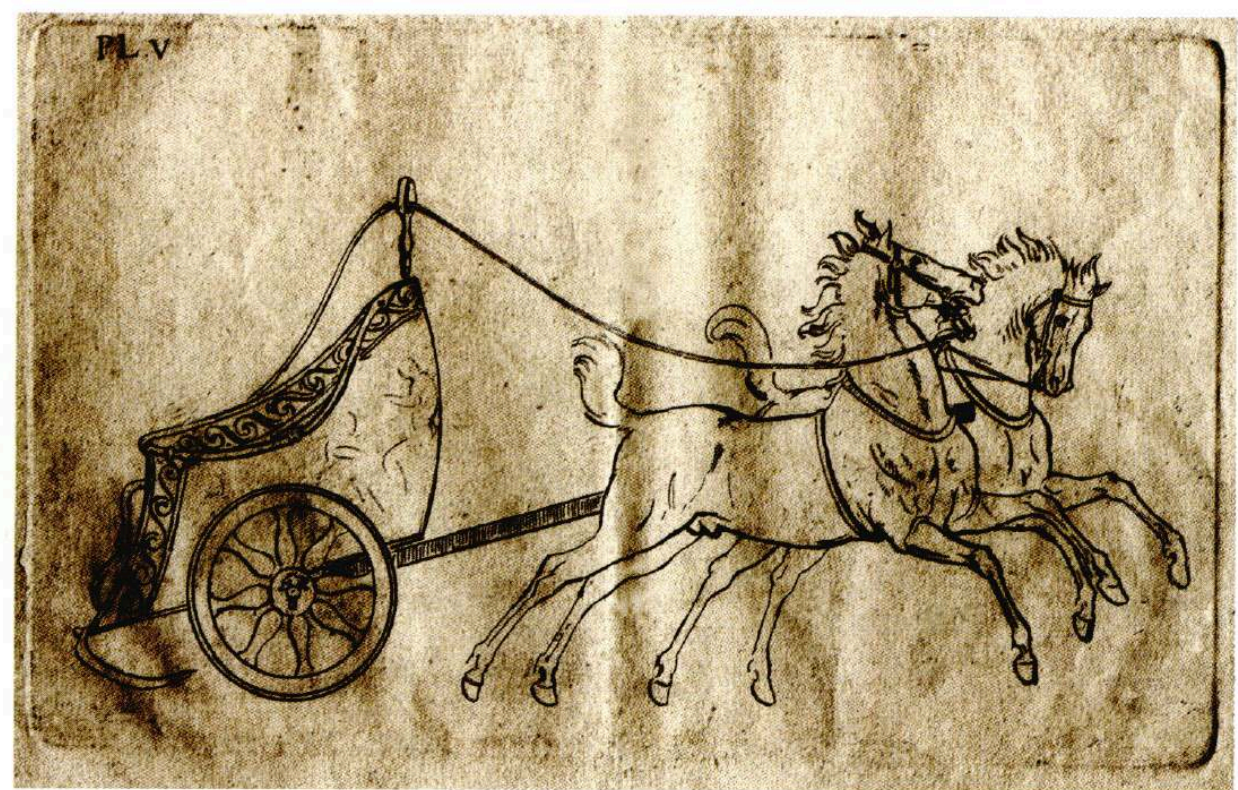

52-Planche V, reconstitution du char en 1818 (Collection privée)
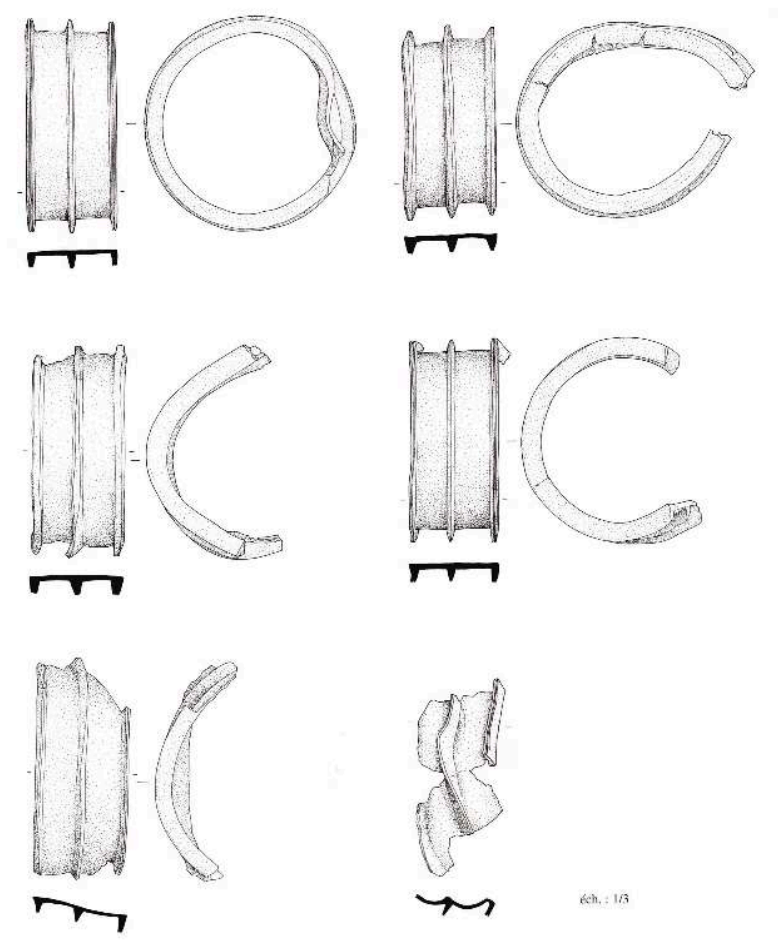

53- Des frettes de moyeux (bronze) 


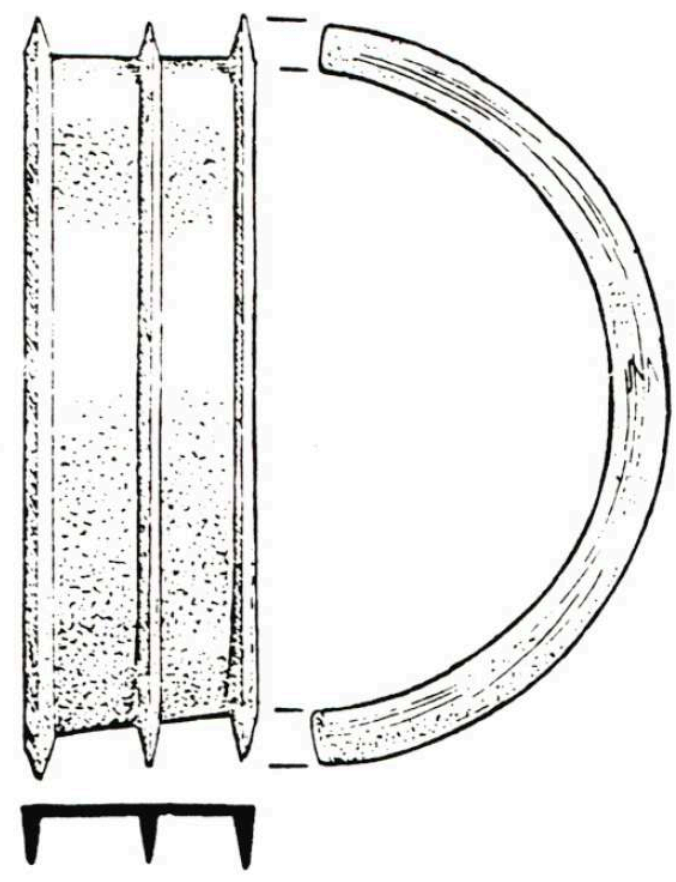

a

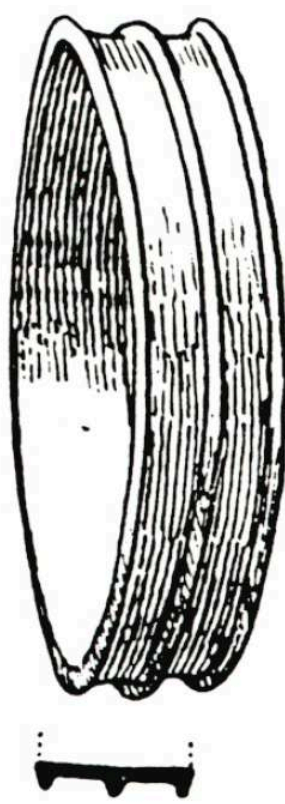

b

54-Frettes de moyeux, a : Armentières-sur-Ourcq (Aisne), d'après Raddatz 1967 : 47 fig. 11, 11 ; b : Armsheim, Kr. Mainz-Bingen, D (La Tène A), d'après Behrens 1927 : 48 fig. 170,12

Deux frettes sont conservées dans leur intégralité (dia. interne 12 et 12,3 cm), alors que d'autres sont quasi complètes (fig. 53). Leur grand nombre témoigne de l'existence d'un char non pas à deux, mais à quatre roues.

Les frettes sont en bronze coulé qui a été retravaillé côté externe du disque ; la surface brute de fonte est conservée à l'intérieur. Les anneaux montrent de légères différences comme les trois nervures, plus ou moins massives qui étaient modelées dans la cire avant la fonte ; enfin, quelques anneaux présentent un sillon sur la crête. A l'intérieur de ces sillons, ont été identifiés des restes d'émail rouge, devenus vert clair par l'oxydation de cuivre des frettes à la surface. Une analyse au microscope électronique à balayage (ZEISS DSM ; 23/03/2000) par S. Greiff (RGZM) à l'Université Gutenberg de Mayence a indiqué surtout du silicium comme produit de décomposition de l'émail dans la cannelure.

La largeur des frettes de 6,1 à 6,3 cm n'est pas induite par des considérations techniques, 2 à $4 \mathrm{~cm}$ étant suffisants ${ }^{138}$, mais est l'expression d'un souci de décoration. Le diamètre des frettes $(12 \mathrm{~cm})$, qui est aussi celui des moyeux de roues permet de restituer un essieu d'un diamètre d'environ $6 \mathrm{~cm}$.

Les frettes coulées à trois nervures sont rares pour l'époque de La Tène (Schönfelder 2002: 150-155). L'exemplaire le plus proche est un fragment d'une tombe d'Armentières-sur-Ourcq (dép. Aisne) (Hubert 1902: 203 sqq. fig. 35 ; Mariën 1961 : 176-177 fig. 68), qui date de La Tène D1 (fig. 54 a). Les autres frettes de moyeux ne possèdent pas de nervure, et pourraient, elles aussi, avoir été émaillées. De l'émail a ainsi été observé sur la crête des frettes en fer de Boé (dép. Lot-et-Garonne) (Schönfelder 2002: 108). Les frettes en bronze de la tombe à char de La Tène $\mathrm{A}$ d'Armsheim (Kr. Mainz-Bingen, D) (fig. 54 b) sont également très proches, mais la 
datation est trop éloignée pour évoquer des relations directes. Les frettes de moyeux de Kappel (Kr. Biberach, D) (Fischer 1959 : 24-25. $\mathrm{n}^{\circ}$ 10-14; Wieland 1996 : 104-105) et de Dejbjerg (Ringkøbing amt, DK) (Petersen 1888, pl. 1,4, 1a) ont des nervures de section triangulaire. Les éléments du char du dépôt de Kappel (La Tène D1) appartiennent aussi à un autre char à quatre roues : cinq frettes de moyeux sont plus qu'il est nécessaire pour deux roues. Les deux chars de Dejbjerg sont aussi des véhicules à quatre roues; l'un est un char celtique (fig. 55), l'autre est une imitation locale, pour lequel peu de fragments ont été retrouvés dans un marais.

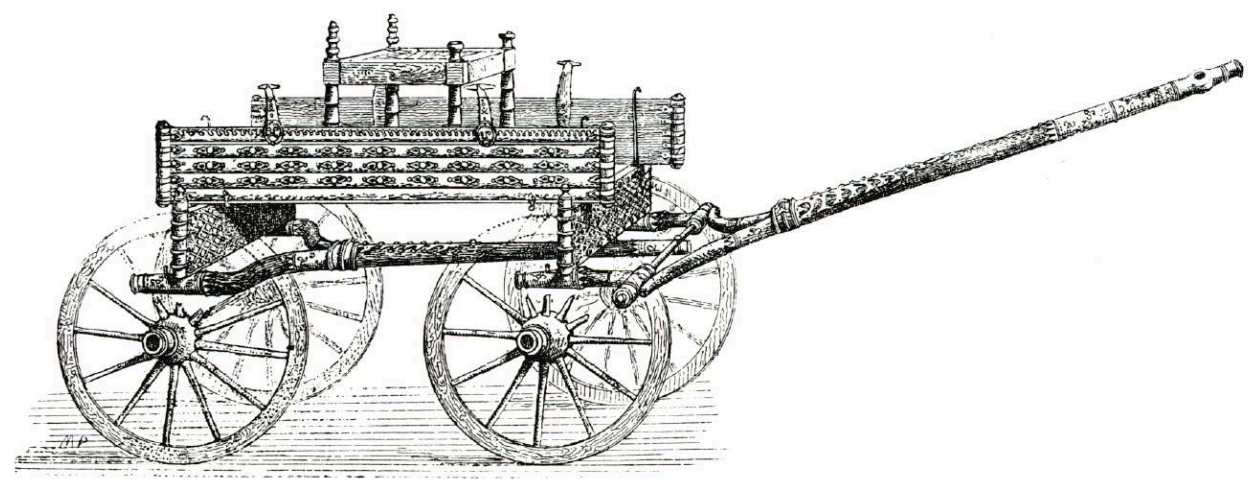

55-Dejbjerg,Ringkøbing amt, DK. -Le char celtique, d'après Petersen 1888 : 30

Dans le mobilier de Verna, cinq tôles de fer pourraient être interprétées comme des agrafes de jantes. Il s'agit de tôles rectangulaires de 6 à $8 \times 20$ à $20,5 \mathrm{~cm}$. (fig. 84). Chaque angle porte des clous de 4,3-4,6 cm de longueur avec une tête hémisphérique. Ces tôles ont été repliées en leur centre autour d'un élément (en bois?). Deux de ces tôles ont été pliées à angle droit.

L'interprétation comme agrafe de jantes, plus faibles normalement, ne peut être retenue : ainsi, les agrafes de jantes décorées de Dejbjerg (Petersen 1888 pl. 1,2 ; 4, 1c) ou de Husby (Kr. Flensburg, D) (Raddatz 1967 pl. 3,5-8) sont très différentes et les agrafes simples de Manching (Lkr. Pfaffenhofen a. d. Ilm, D) - qui n'étaient pas associées à un char - sont plus en forme de bande (Jacobi $1974: 211$ sqq. ${ }^{\circ}$. 828-829). Enfin les clous de Verna sont trop massifs pour ce type d'objet. Ces tôles de fer seront donc placées avec les autres objets de fonction inconnue (cf. infra).

\section{LES CLAVETTES DU CHAR}

Quatre clavettes de deux types différents coexistent. Une première paire de clavettes est en fer, à tête en demi-lune avec une tige courbée (fig. 56). La tête est accentuée par une nervure à l'intérieur vers l'essieu, connue aussi dans l'ensemble funéraire (à quatre chars) de La Mailleraye-sur-Seine (dép. Seine-Maritime) daté de la transition La Tène C2/D 1 (fig. 57) (Lequoy 1993 : 130, fig. 12, 35). Une datation assez lâche entre La Tène C et $\mathrm{D}$ est habituellement proposée pour ce type de clavette.

Les exemplaires de Verna se distinguent des exemplaires connus par leur section octogonale, par les deux perforations dans la tête en demi-lune ${ }^{139}$ et par le bouton final travaillé très minutieusement. L'étude des chars à deux roues nous apprend que les tiges des paires de clavettes étaient courbées dans deux directions différentes ${ }^{140}$. Par conséquent, ces deux clavettes à tête en demi-lune de Verna appartiennent au même essieu. 
La deuxième paire de clavettes est à tête décorée en bronze et à tige droite. Une des têtes étant fondue (fig. $\mathbf{5 8}$ b), on a pu observer sa structure en fer de support en T. L'autre exemplaire dessiné en 1986 n'est plus à Hières-sur-Amby (fig. 58 a). La forme basique de la tête est en demi-lune percée par trois trous avec des crochets aux bouts alors qu'une appendice en forme de fleur est visible sur le devant.

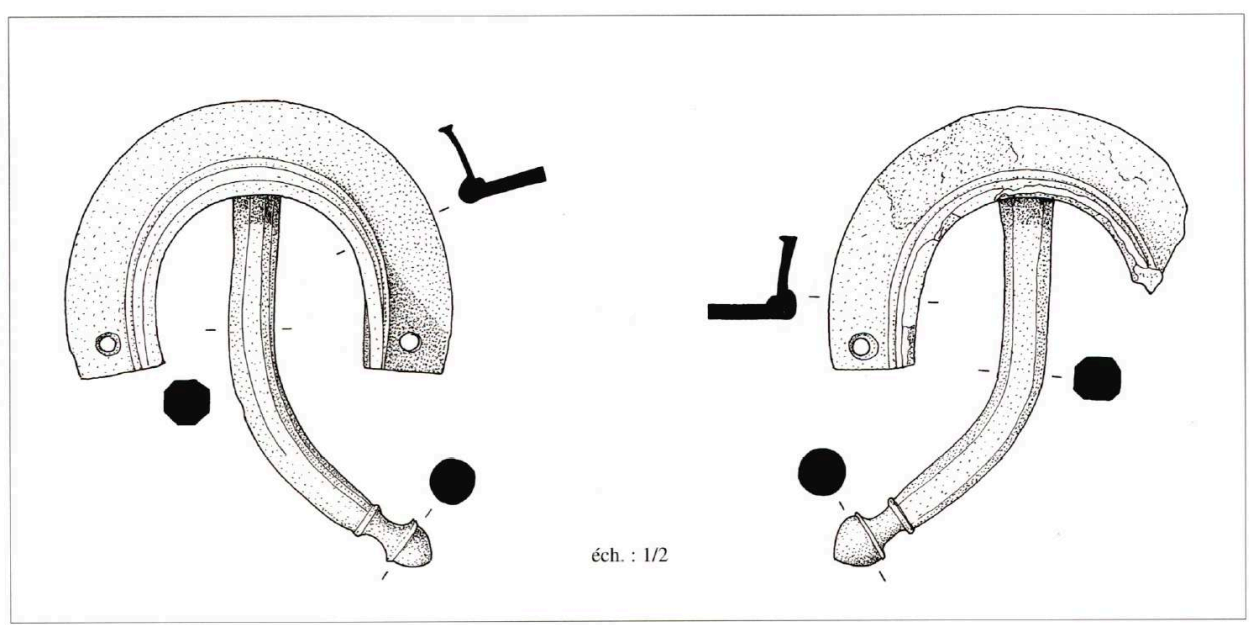

56-Paire de clavettes (fer)

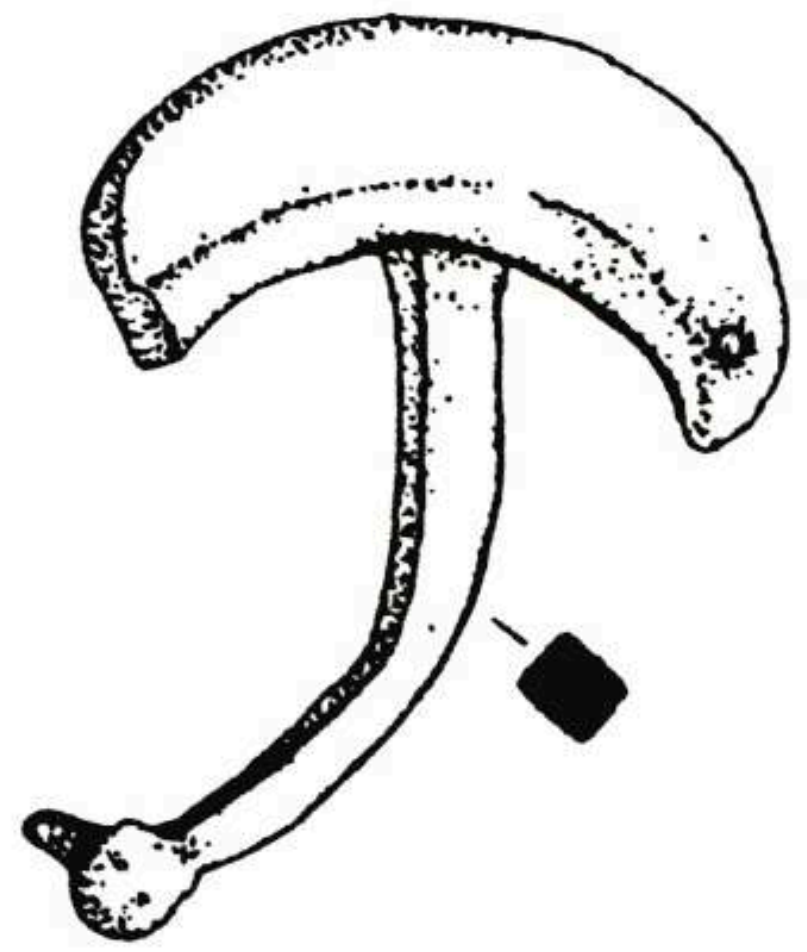

57-La Mailleraye-sur-Seine, (Seine-Maritime), d'après Lequoy 1993 :130, fig. 12,35. -(fer)

La typologie de clavettes à tête décorée en bronze n'est pas très précise et l'on trouve rarement des parallèles directs. On mentionnera une clavette du Rhin près de Mayence (fig. 59) ${ }^{141}$; sa tête en bronze et en demi-lune est structurée par les trois trous. 
Fixés dans l'essieu, les deux types, différents, ne se distinguent guère. On peut calculer un diamètre de l'essieu de 5,8 cm maximum, grâce au diamètre interne de l'espace inclus par les têtes en demi-lune.

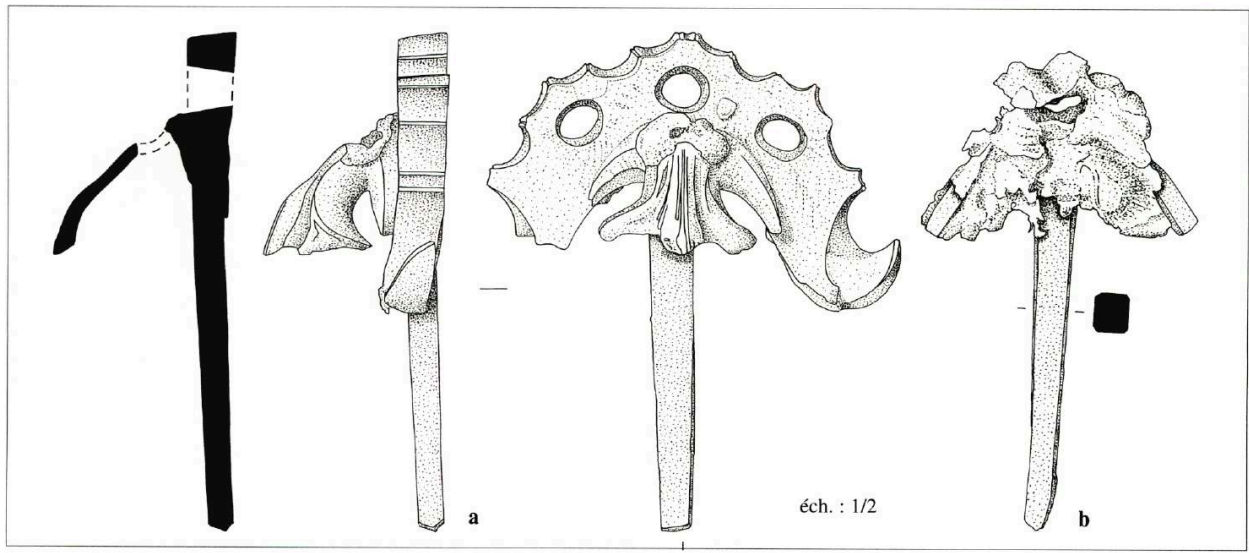

58 a et b - Paire de clavettes (bronze et fer)

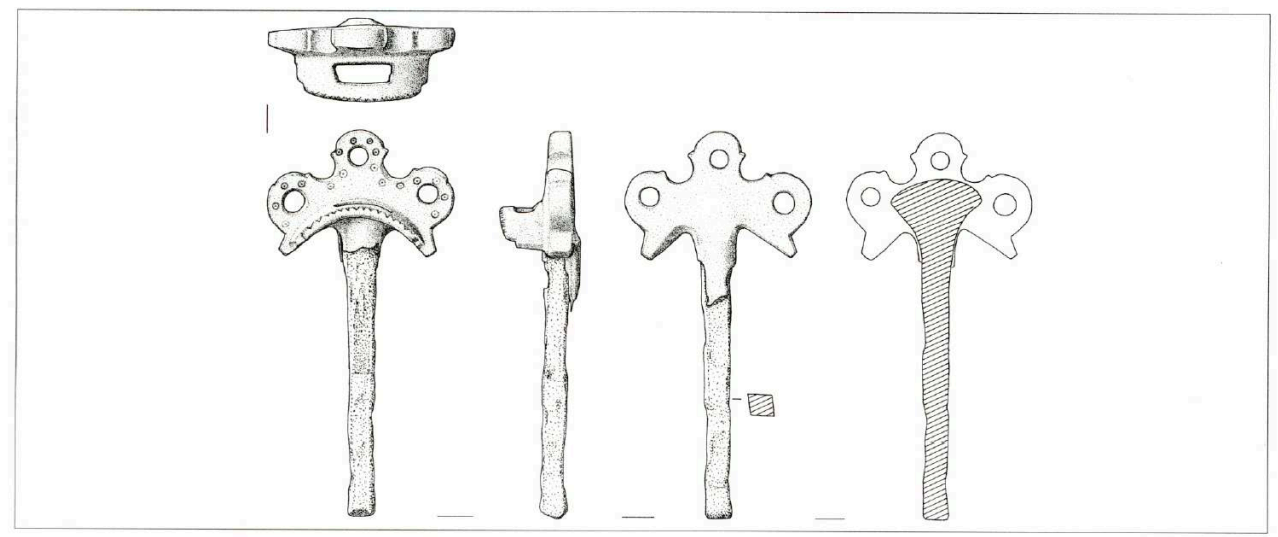

59 - Clavette du Rhin près de Mayence (bronze et fer)

\section{ÉLÉMENTS DE LA STRUCTURE INFÉRIEURE DU CHAR}

La rotation de l'essieu avant et des branches de traction par rapport à la caisse du char est rendu possible par une tige pivot (fig. 60). Cette tige était en bois pour les chars à quatre roues du Hallstatt ${ }^{142}$ ainsi que pour ceux de la vie quotidienne du deuxième âge du Fer. Ainsi, aucune tige de pivot en fer n'est connue pour les chars de Boé, Dejbjerg ou Husby. Le seul exemple en fer pour la protohistoire demeure celui de Verna (fig. 61 a) ; (long. 28,0 cm; dia. tête $4 \mathrm{~cm}$ ). Pour le début de l'époque impériale, des tiges de pivot comparables sont connues dans des camps militaires ${ }^{143}$. 


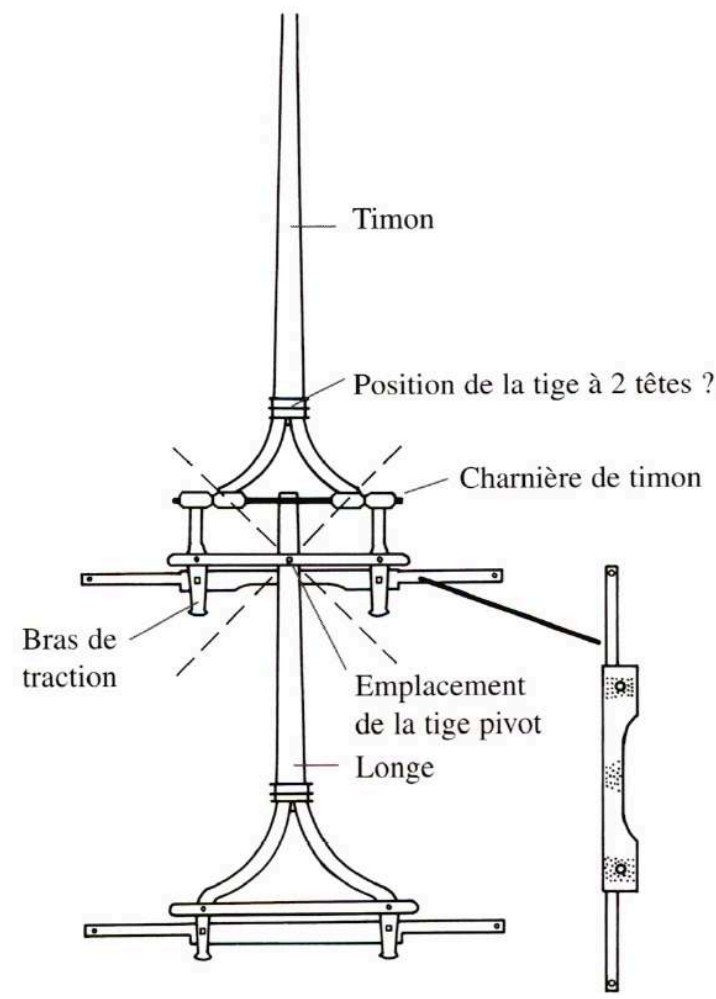

60 - Dejbjerg, le char celtique. Eléments techniques de la structure inférieure, d'après Hayen 1983 : 458 fig. 16

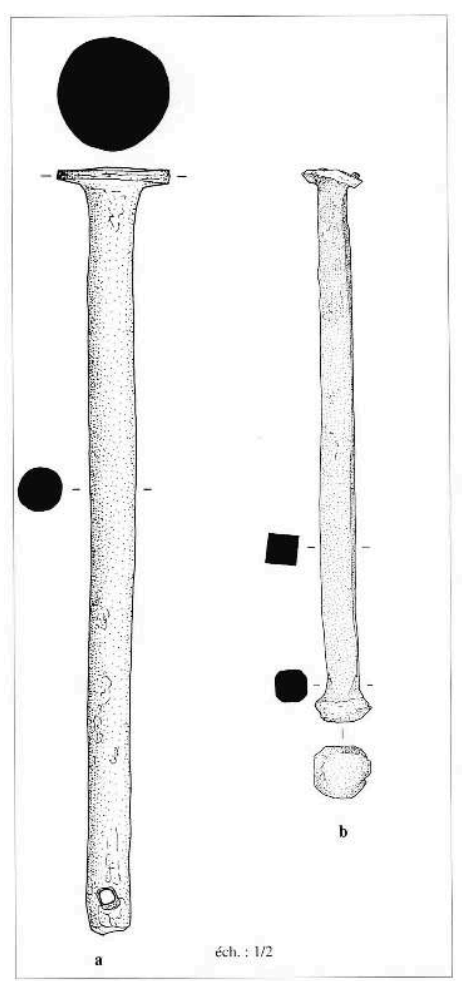

61 a -Tige de pivot ; $b$ : tige à deux têtes ( $a, b$ fer) 
Les sources archéologiques pour ces chars méditerranéens étant trop lacunaires, rien ne peut venir expliquer l'origine de ces tiges de pivot en fer. L'origine méditerranéenne demeure donc une hypothèse.

Citons une tige de pivot comparable à celle de Verna à Manching, trouvée au détecteur de métaux hors contexte (Jacobi 1974, pl. 72 nº 1358 [Inv. 1963/1257]), mais une datation romaine voire postérieure est possible pour cet exemplaire, les tiges de pivot n'ayant guère évolué. Les goupilles modernes pour atteler les remorques sont d'ailleurs identiques. Enfin, une tige de pivot de l'oppidum "La Pierre d'Appel" à ÉtivalClairefontaine (dép. Vosges) fait partie du niveau I, daté entre La Tène D2 et l'époque augustéenne (Deyber et al. $1984: 210$ fig. 26, $3: 214-215$ ).

8 A ce jour, seules trois tombes à char ont livré des tiges en fer avec des têtes forgées sur les deux côtés. Celle du char de Verna, qui appartient à la catégorie des chars à quatre roues de La Tène D1, mesure $20,3 \mathrm{~cm}$ de longueur et la tige à section carrée est épaisse de $1 \mathrm{~cm}$ (fig. $\mathbf{6 1}$ b). Une des têtes est forgée minutieusement dans une matrice, l'autre est plate ${ }^{144}$.

9 Un élément comparable provient d'une tombe à incinération avec éléments de joug de la nécropole de la Croix du Rebout au Mont Beuvray (dép. Saône-et-Loire/Nièvre) ${ }^{145}$; une tige à deux têtes de $13 \mathrm{~cm}$ de long fait partie d'une tombe à char à deux roues de La Tène B de Pont-Faverger/La Wardelle (dép. Marne) (Dupuis 1940:63-64, fig. 2). Ces tiges n'ont jamais été trouvées en place à la fouille. On observe que la tête plate ou forgée ne forme jamais un angle droit avec la tige. Il est possible que cette tige de section carrée fixe plusieurs éléments en bois de manière statique. On a introduit la tige incandescente avec une tête préfabriquée dans le trou percé, et l'autre tête a été forgée sur place. Les dimensions permettent de fixer le timon ou la longe écartée du char avec des structures en $\mathrm{Y}$ ou en $\mathrm{YY}^{146}$. Pour le timon ou la longe, l'utilisation des tiges à double têtes ne renseigne pas sur la présence de un ou de deux essieux. A Dejbjerg, Boé et Husby ce sont des bagues ovales de formes différentes qui fixent les bois fendus du timon et de la longe ${ }^{147}$.

Deux groupes d'objets peuvent être associés au timon. Le premier est un disque convexe en tôle de bronze de $5,8 \mathrm{~cm}$ de diamètre, qui était fixé avec trois petits clous alignés en bronze sur un autre objet (fig. 62 a). De plus, deux bagues en fer (dia. max. externe 7,1-7,6 cm ; largeur $1,1 \mathrm{~cm}$ ) ; (fig. 62 b) possèdent un diamètre un peu plus grand que celui de ce disque en bronze.

L'absence de comparaison dans d'autres tombes à char ou en contexte d'habitat, ne permet pas d'appréhender leur fonction. Les indices pour attester de l'existence d'un char à quatre roues à Verna sont suffisamment nombreux. On peut certes essayer de chercher des interprétations techniques comme leur utilisation à l'extrémité des essieux. D'autre part les chars à quatre roues ont une charnière de timon très fragile. L'axe de cette charnière est la tige en bois la plus vulnérable du char, qui résiste aux forces de traction de deux chevaux. Les chars hallstattiens ont souvent des fixations supplémentaires avec des anneaux ${ }^{148}$ et les deux bagues pourraient alors avoir la même fonction. Une autre hypothèse serait celle de deux fronts de la charnière de timon décorés avec des disques bombés en bronze. 


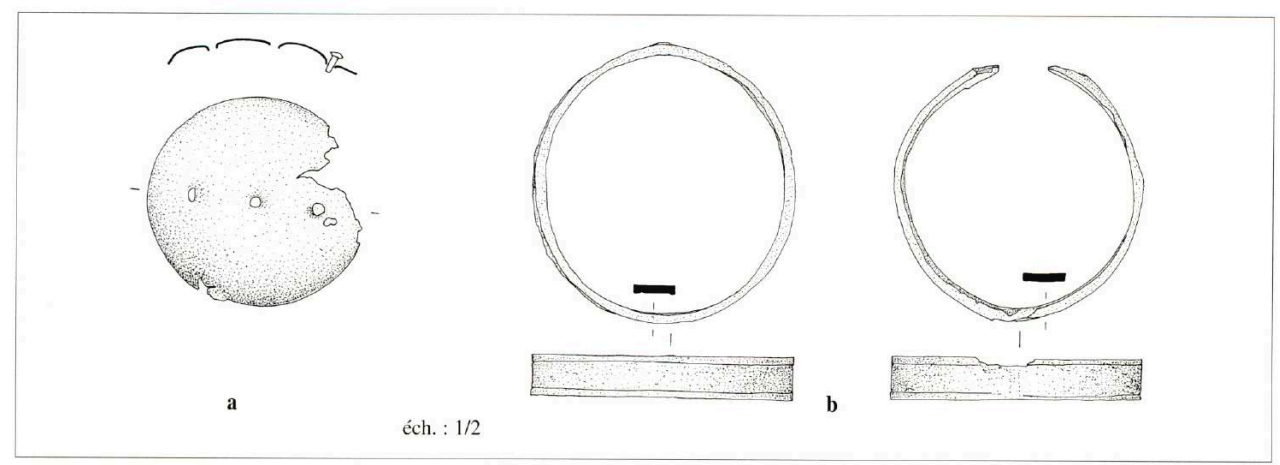

62-Garniture de la charnière de timon (?) (a : bronze, b : fer)

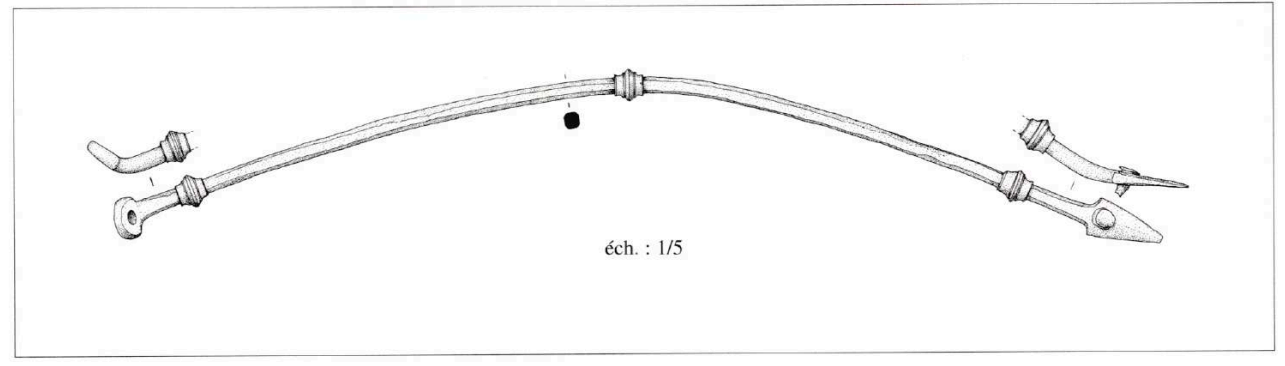

63-Tige de la structure inférieure du char (fer avec bronze)

Deux longues tiges en fer de 71,7 et $69 \mathrm{~cm}$ de long font également partie de la collection (fig. 63). De petits éléments décoratifs en bronze (long, environ 2,4 cm), à profil de scotie / tore / scotie sont visibles au milieu ainsi qu'aux extrémités des tiges, l'une pointue, l'autre arrondie, toutes deux courbées pour être fixée l'une par un clou, l'autre par deux clous.

193 Aucune comparaison n'existe avec les chars à deux ou à quatre roues de l'Europe tempérée mais par contre des chars romains possèdent des éléments semblables comme supports de la caisse ${ }^{149}$.

\section{LA CAISSE}

Apparemment, la caisse du char était richement décorée. Aujourd'hui l'état de conservation du mobilier ne permet pas de reconstitution crédible. Du fait de l'incinération, aucune trace de bois n'est conservée sur le revers des objets. De plus, les objets métalliques ont fait l'objet d'un tri lors de la cérémonie funéraire. L'attribution à la caisse reste, pour la plupart des objets, le résultat d'études comparatives avec d'autres véhicules.

Nous avons montré que les deux longues tiges (fig. 63) faisaient probablement partie de la structure inférieure du char. Deux autres tiges avec des décors similaires (fig. 64) étaient fixées sur un élément en bois massif (long. 40,4 et 30,3 cm). Elles sont courbées en double $\mathrm{S}$, de section octogonale et également décorées : trois éléments en bronze avec un profil de scotie / tore /scotie. Ces tiges fixées sur le bois avec deux clous de chaque côté, sont décorées de la même manière que les grandes tiges. Ce sont probablement les poignées de la caisse ; leur position est incertaine. Deux comparaisons existent à Bern/Tiefenau (canton de Bern, $\mathrm{CH}$ ) (Müller $1990: 239$ pl. 21, 143) et à Mons/ 
Mont Eribus (Prov. Hainaut. B) (Mariën 1961 : fig. 37, 48). Ces objets sont là sans contexte, mais ils sont associés à un grand nombre de pièces de char.

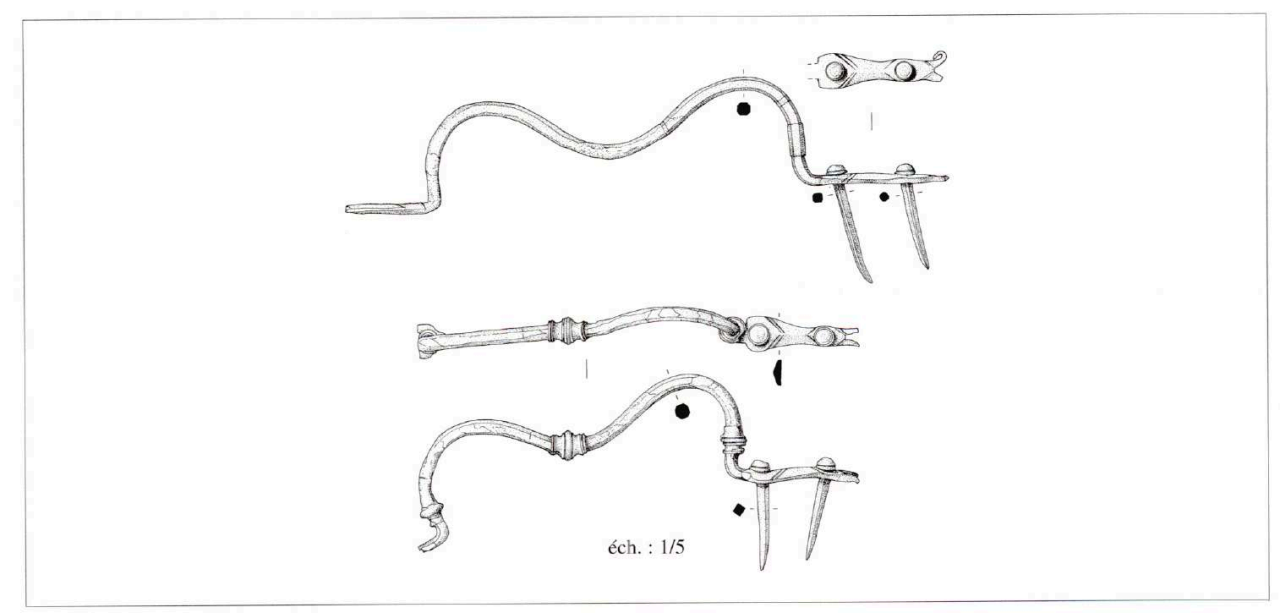

64-Poignées (fer et bronze)

Deux agrafes (long. 16,0/15,7 cm, larg. 2,7 cm (sans les crochets) sont aussi décorées d'une manière analogue (fig. 65 a et b). Elles étaient donc aussi utilisées pour le char, mais aucune fonction précise ne peut leur être attribuée. Trois dernières agrafes plus longues et sans décor ne peuvent être attribuées assurément au char, mais par contre correspondent à des fixations utilisées en menuiserie ( $c f$. infra).

Deux groupes de pitons à oeil aux extrémités rabattues avec des anneaux insérés font partie de la décoration latérale de la caisse. Les pitons à œil sont les éléments constitutifs des structures inférieures des chars à deux roues laténiens ${ }^{150}$, à raison de deux ou quatre exemplaires dans les tombes concernées. A Verna, ils sont plus nombreux : huit grands exemplaires (fig. 66 a), supportés par des plaques en forme d'amande (long. 13,4-15,0 cm ; larg. 4,1-5,1 cm), (dia. de l'anneau 7,2-9,0 cm ; long, du piton 4,6 cm) et sept petits (fig. 66 b), (dia. de l'anneau 3,5-3,7 cm ; long, du piton 3,2 $\mathrm{cm}$ ). Les anneaux des petits pitons sont faits d'une bande de bronze (larg. $0,8-1,1 \mathrm{~cm}$ ), quelques-uns sont brûlés; dans un cas un piton est inséré dans une tôle en bronze décorée (fig. 67).

Six anneaux sont attestés pour le char à deux roues de la tombe 6 de Bescheid "Bei den Hiibeln" (Kr. Trier-Saarburg, D) (Haffner 1992 : 49 fig. 23). Cette tombe est datée de La Tène A. Le plan de la fouille montre qu'un décor de caisse est probable dans cet exemple.

199 A Verna, il existe un grand nombre de tôles décorées (fig. 67), liées par cet anneau en bronze et le piton avec les autres éléments décoratifs du char. Il est impossible de comprendre le fonctionnement de ces tôles décoratives. La restauration n'a pas révélé l'organisation du système ornemental. Il existe des décors de Ying/Yang de taille différente, liés par des lignes et des triangles courbés; parfois les tôles sont ajourées. Deux tôles sont presque rectangulaires, avec une ligne repoussée au bord. Leurs fonctions ne sont pas claires, mais en tout cas elles ornaient un objet très complexe. 
107

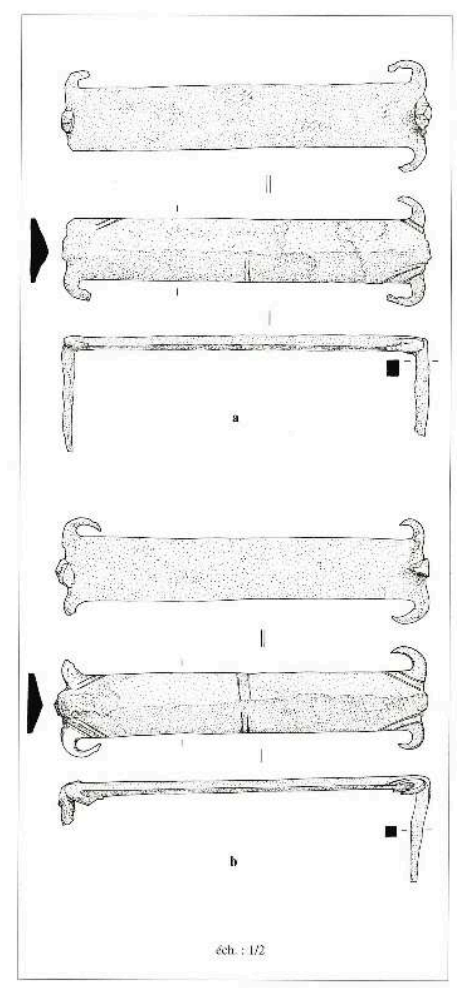

65 a et b - Dux agrafes décorées du char (fer)

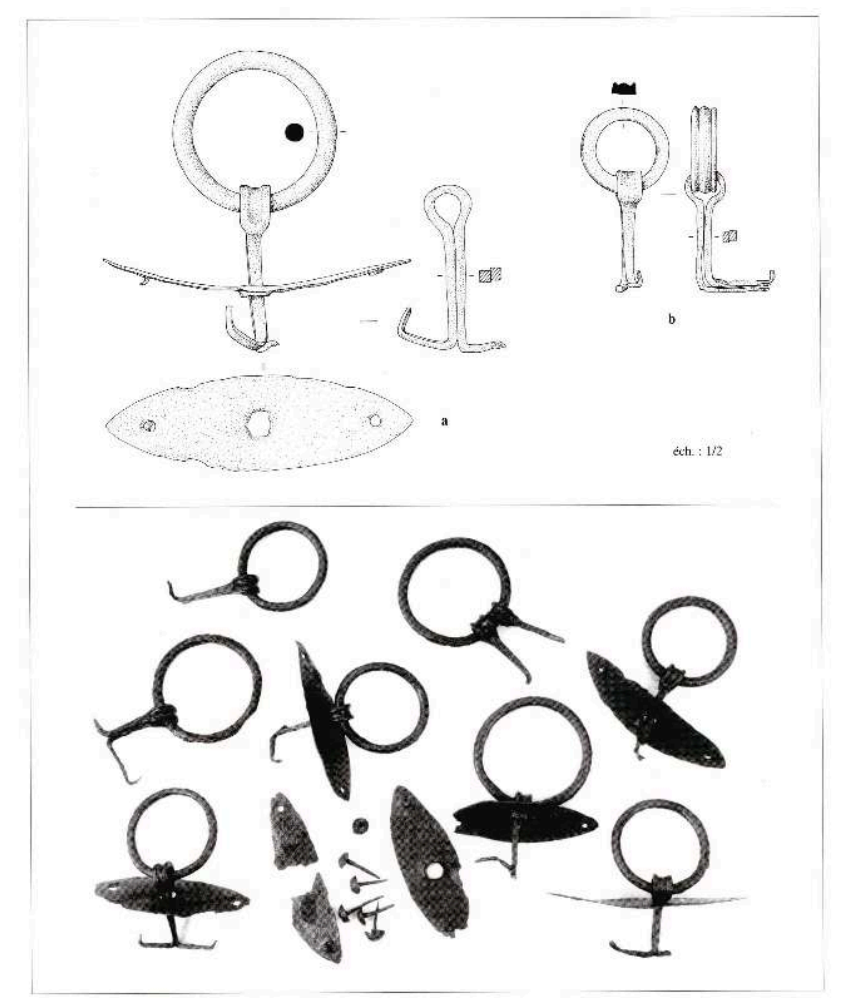

66 a et b - Pitons à til aux deux boots raattus ( $a$, fer ; b, fer et bronze) 


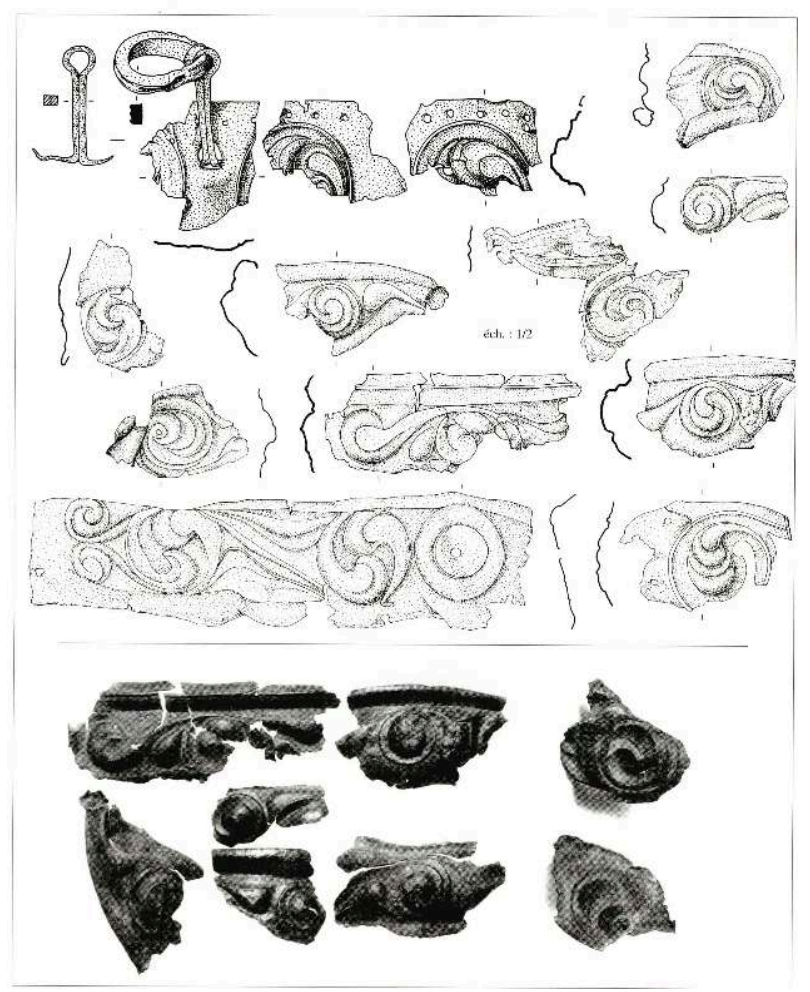

67- Tôles décoratives (bronze)

\section{LE JOUG}

Deux anneaux profilés en bronze avec langue de fixation sur un côté sont des anneaux passe-guides. Ceux-ci sont fixés sur le joug, permettant de conduire les deux chevaux. Un des anneaux porte des traces de feu, l'autre est intact (larg. 5,7 cm; haut. 5,1 cm) (fig. 68). Ce type d'anneau se distingue d'autres types fixés sur le joug par un étrier de fixation et sous une décoration couvrant une petite partie de la surface du joug (voir les types Estinnes, Bechtheim, Kappel, Alésia et autres ${ }^{151}$ ).

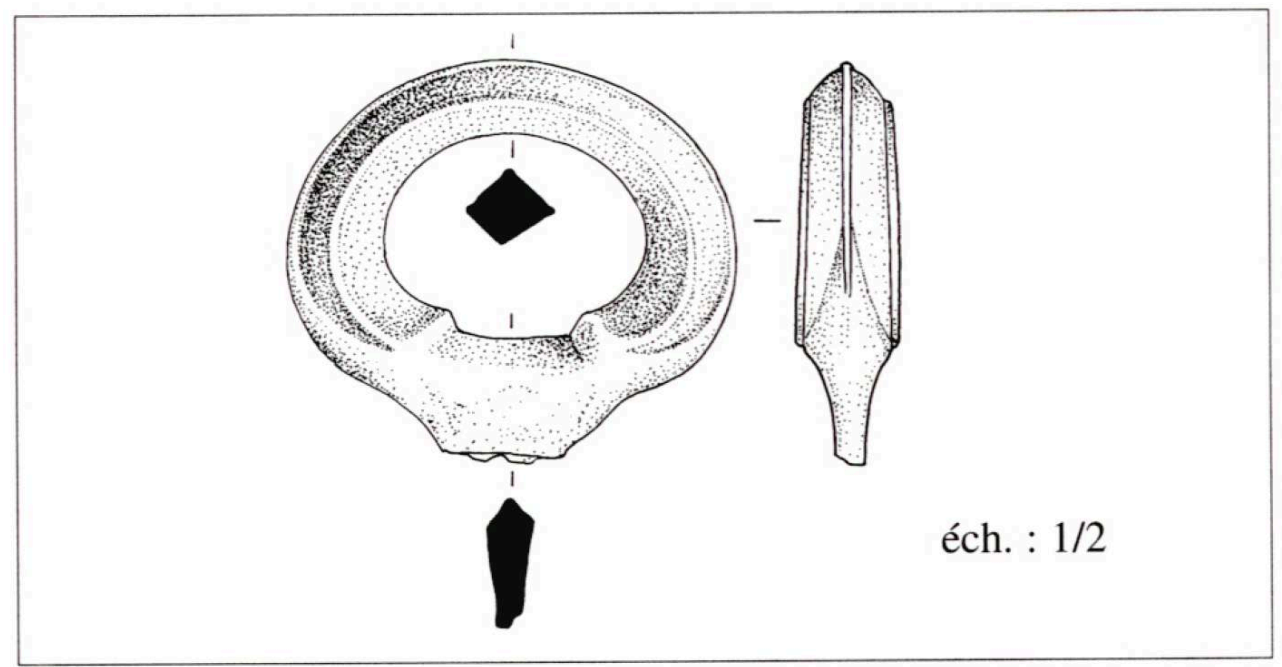

68-Anneau passe-guide (bronze) 
Ces anneaux passe-guides sont assez rares, sans contexte daté, et ont été découverts dans des habitats. L'exemplaire de Verna devient donc éponyme (tab. 14) ${ }^{152}$. L'anneau de la tombe 2 de Kollig (fig. 69 c) en forme de fer à cheval avec languette de fixation et clou unilatéral est le seul en fer; les autres sont en bronze. La répartition ne privilégie aucune région.

D'autres éléments du joug posent plus de problème comme un chapeau en bronze avec une grande tête hémisphérique émaillée (haut, total 9,3 cm, dia. tête $3,4 \mathrm{~cm}$ ), fortement brûlée (fig. 70 a). Reste seulement le décor strié, l'émail ayant fondu dans le bûcher. Un côté du chapeau semble être ouvert, sa surface est décorée avec plusieurs stries verticales. Un petit trou témoigne d'une fixation sur bois avec des clous. Une deuxième tête hémisphérique émaillée (dia. tête $2,9 \mathrm{~cm}$ ) atteste probablement l'existence d'un deuxième chapeau (fig. $\mathbf{7 0}$ b) qui a perdu son corps. Deux autres clous en bronze à tête émaillée (dia. 1,8/1,8 cm, haut. 1,2/1,0 cm) ont décoré le joug ou la caisse du char (fig. $70 \mathrm{c})$.
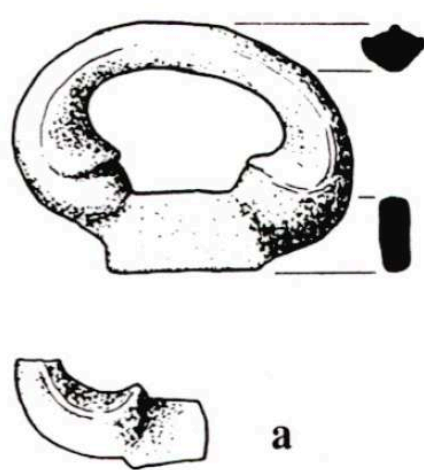

a

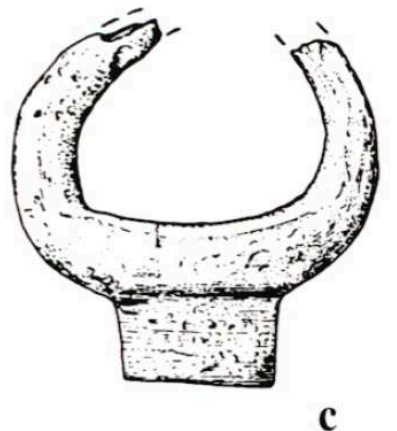

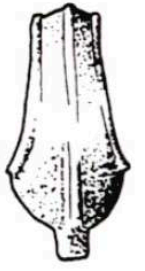

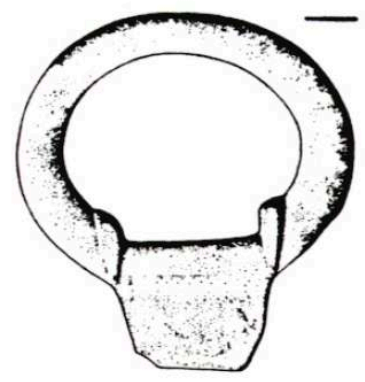

b

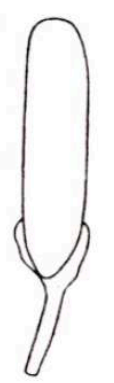

éch. : $1 / 2$

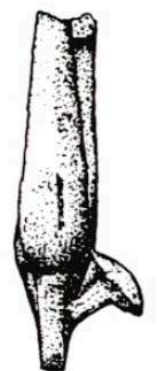

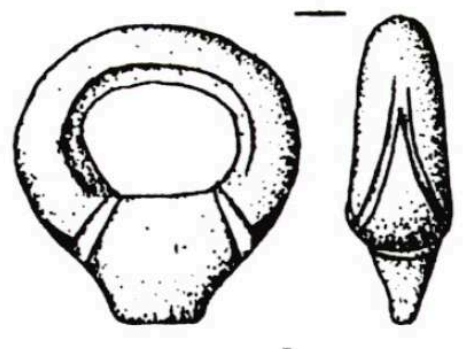

d

69 a -Levroux/Colline des Tours (Indre) ; b : Oberursel-Oberstedten / Heidetränke (Hochtaunuskreis, D) (bronze) ; c : Kollig Grab 2 (Lkr. Mayen-Koblenz, D) (fer) ; d : Manching (Lkr. Pfaffenhofen a. d. IIm, D) (bronze) 


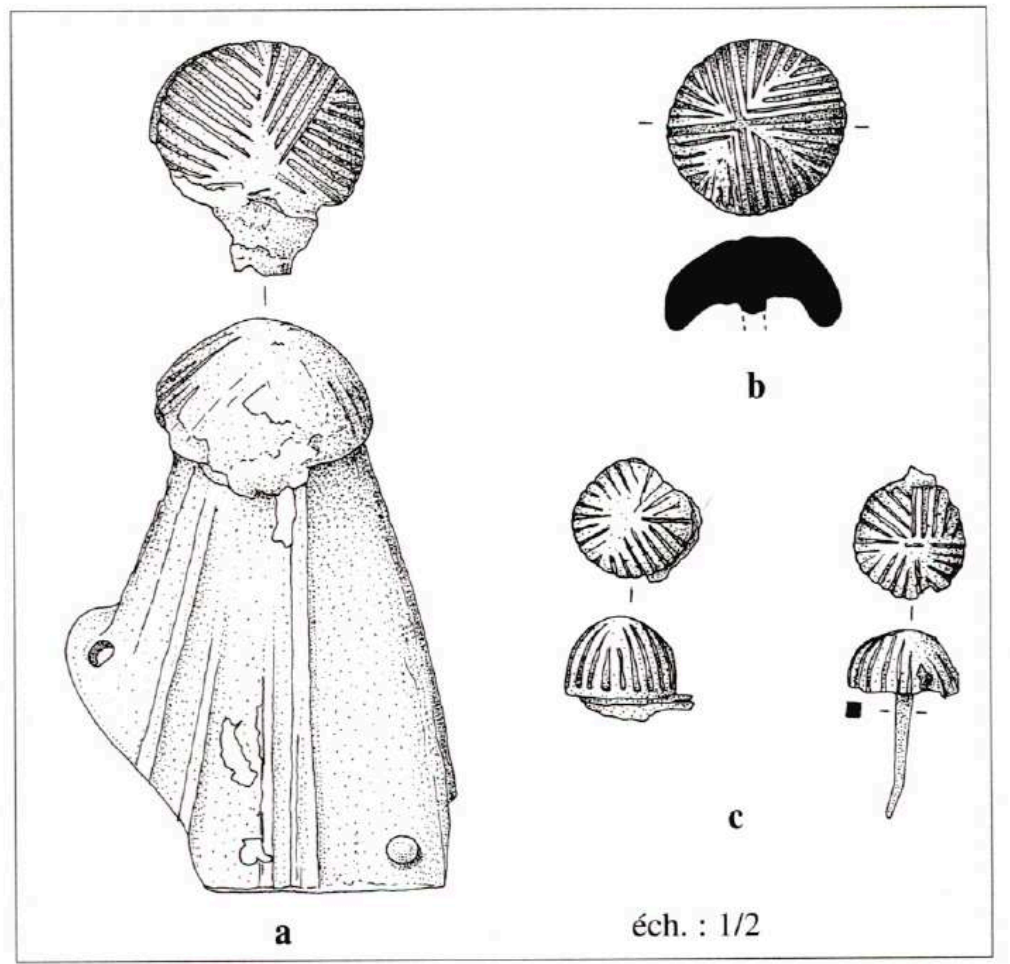

70 a -Chapeau du joug (?) à tête hémisphérique (bronze) ; b : tète hémisphérique (bronze) ; c : clous (bronze)

Ces deux chapeaux peuvent avoir orné le joug sur les deux côtés, comme les chapeaux de type Orešak ${ }^{153}$. Mais la forte dégradation du mobilier de Verna ne permet pas de trancher entre un emplacement sur le joug ou aux coins de la caisse du char.

\begin{tabular}{|c|c|c|c|c|}
\hline Lieu de découverte & $\begin{array}{l}\text { Nombre/ } \\
\text { Description }\end{array}$ & Contexte & Datation & Bibliographie \\
\hline Verna (Dép. Isère, F) & $\begin{array}{l}2 \text { ex., l'un en } \\
\text { fragment }\end{array}$ & $\begin{array}{l}\text { tombe avec char } \\
\text { à quatre roues }\end{array}$ & Lt D1 & \\
\hline $\begin{array}{l}\text { Kollig, tombe } 2 \\
\text { (Lkr. Mayen-Koblenz, D) }\end{array}$ & 1 ex. fer & $\begin{array}{l}\text { tombe, pars pro toto } \\
\text { pour un char }\end{array}$ & Lt D & $\begin{array}{l}\text { Haffner, Joachim } 1984: 82 \text {, } \\
\text { fig. } 11,9 .\end{array}$ \\
\hline $\begin{array}{l}\text { Levroux/Colline des Tours } \\
\text { (Dép. Indre, F.) }\end{array}$ & $\begin{array}{l}2 \text { ex., l'un en } \\
\text { fragment }\end{array}$ & $\begin{array}{l}\text { oppidum (trouvaille } \\
\text { de prospection) }\end{array}$ & - & $\begin{array}{l}\text { Buchsenschutz et al. } 1988 \text { : } \\
106 \text { fig. } 71 .\end{array}$ \\
\hline $\begin{array}{l}\text { Oberursel-Oberstedten / } \\
\text { Heidetränke (Hochtaunuskreis, D) }\end{array}$ & 1 ex. fer & oppidum & - & $\begin{array}{l}\text { Müller-Karpe, Müller-Karpe } \\
1977: 50 \text { fig. 4, } 10 .\end{array}$ \\
\hline $\begin{array}{l}\text { Manching (Lkr. Pfaffenhofen } \\
\text { a. d. Ilm, D) }\end{array}$ & 1 ex. fort profilé & $\begin{array}{l}\text { oppidum (trouvaille } \\
\text { de prospection avec } \\
\text { détecteur) }\end{array}$ & - & $\begin{array}{l}\text { Riedel 1993/94 : } 252 \\
\text { fig. } 5 .\end{array}$ \\
\hline $\begin{array}{l}\text { Soissons } \\
\text { (Dép. Aisne, F) }\end{array}$ & 1 ex. & tombes & - & $\begin{array}{l}\text { Aimable renseignement de } \\
\text { D. Roussel, Musée de Soissons. }\end{array}$ \\
\hline
\end{tabular}

Tableau 14 -Anneaux passe-guides du type Verna

\section{CONCLUSION}

Le char de Verna est un des rares exemplaires d'un véhicule à quatre roues du second âge du Fer ${ }^{154}$. Les chars à quatre roues étant assez rares, objets de prestige d'une longue tradition depuis l'âge du Bronze ${ }^{155}$, ils revêtent certainement une importance symbolique liée au pouvoir à La Tène Finale. De plus, le char de Verna est un véritable char de parade celtique, car les frettes et les clavettes sont décorées en bronze et la 
caisse est ornée de tôles et d'anneaux. Plusieurs parties montrent des influences romaines, comme la construction de l'essieu tournant à piton en fer et les tiges décorées.

Cette synthèse entre celtique et romain correspond à la position géographique de la tombe et à la datation, donnée par les cabochons émaillés et la céramique. A La Tène D1b, entre 100 et 80 av. J.-C., l'oppidum de Larina et la sépulture du tumulus de Verna, faisaient partie de la Provincia. Le défunt de Verna a utilisé son char pour une démonstration officielle de son pouvoir, dont nous ne connaissons pas l'origine. L'entourage ou la personne au pouvoir après son décès a choisi de le laisser brûler et enterrer avec les restes de cet objet de prestige. L'utilisation d'un char traditionnel, qui reflète d'une certaine manière encore les traditions hallstattiennes, indique les besoins de l'élite de se rattacher à (ou de créer) des bases de pouvoir traditionnelles.

Grâce aux découvertes des tombes de Verna et de Boé, on peut attester que les chars à quatre roues appartiennent aux tombes les plus riches de leurs époques. Par contre, les chars à deux roues se retrouvent dans de nombreuses autres tombes associés à des objets funéraires variés. Malgré tout la présence d'un char à deux roues dans une sépulture relève d'une sorte de privilège, dont on ne connaît pas les raisons précises. Quelques personnes ont le droit d'utiliser les chars, les autres pas. L'absence de chars à quatre roues dans les tombes au centre de la zone des oppida, de la Gaule à la Bohème, est explicable par les rites funéraires. Ils ne donnent pas en effet une illustration représentative de la vie et de l'utilisation des objets de prestige.

Les chars à quatre roues étaient inhabituels dans le monde celtique. Aux marges de la culture celtique, les tombes de Verna et Boé nous donnent deux exemples, avec des contextes funéraires très riches. Les différences entre le noyau et la périphérie du monde celtique, mais aussi une réétude des découvertes nous permettront de développer une image plus variée de l'élite celtique, que celle donnée par les sépultures d'une région unique.

208 La tradition des tombes à char pendant plus de cinq siècles diffère des modes éphémères comme celles d'autres objets de prestige, les torques en or ou les amphores à vin par exemple. Les époques romaine ${ }^{156}$ et mérovingienne ${ }^{157}$, ont repris cette coutume dans certaines régions. Les chars laténiens et les cérémoniels qui y sont associés, sont une des bases fondamentales de la notion de prestige à l'époque gauloise, comme l'or et les armes.

Pendant la République et à l'époque impériale romaine, le droit d'utiliser un char est le signe de certaines fonctions publiques. A Rome, c'était le magistrat qui avait le droit d'utiliser un char dans la ville ${ }^{158}$; sur un petit nombre de sarcophages on trouve des chars de course et des chars à fauteuil ${ }^{159}$. Le char de triomphe comme char héroïsant et archaïsant pour des occasions exceptionnelles n'a par contre rien à voir avec un usage quotidien ou un insigne personnel ${ }^{160}$. Le currus triumphalis est un véhicule haut, particulièrement décoré, et il diffère des chars de cirque légers. Bien que la tombe de Verna était placée dans la province romaine, il est improbable que le char corresponde à un de ces insignes romains.

210 Une restitution du char de Verna est, nous l'avons vu, impossible avec les données laissées par le bûcher et par la documentation de la fouille. Manquent les dimensions de la caisse, des roues et des autres éléments en bois. Le char celtique du Dejbjerg (fig. 55), offre une bonne image des chars à quatre roues. Ce char peut servir d'exemple pour la 
dimension des roues et pour la taille de la caisse. Les positions exactes des tiges longues et des poignées, ainsi que les décors de la caisse, restent hypothétiques.

211 Afin de ne pas donner d'idée, peut-être erronée, nous avons préféré ne pas réaliser de reconstitution. Seules de nouvelles fouilles de tombes à char, où ils n'auraient pas brûlés, pourraient apporter des réponses.

\section{LES ÉLÉMENTS DU HARNACHEMENT. M. Schönfelder}

212 Dans le matériel de Verna, trois mors de bride (fig. 71, 72) et un anneau en fer appartiennent au harnachement; ces objets portent les traces du bûcher. La présence conjointe de trois mors existe dans plusieurs tombes à char ; les chars étant tirés par deux chevaux, le troisième mors est alors attribué à un cheval monté (Frey 1976).

213 La documentation de 1818 mentionne un "attelage de quatre chevaux [sic]", les restes des quatre roues ayant été interprétés comme les vestiges de deux chars car les chars à quatre roues n'avaient alors jamais été découverts lors de fouilles et surtout, les sources écrites de l'Antiquité signalent des chars de guerre, rapides, donc à deux roues.

214 Deux mors de bride semblables, peu décorés, forment une paire (fig. 72 a et c), alors que le troisième, est plus décoré (fig. $\mathbf{7 2}$ b). Les tiges latérales des deux premiers ont une section ronde, et la tige qui sert de gourmette est placée sur une pièce rectangulaire plus épaisse, à trois trous. Le troisième mors a quant à lui des tiges latérales facetées et l'épaississement est composé de trois disques percés. Ces mors, proches des exemplaires modernes, exceptée la gourmette à chaîne, ont apparemment été une attraction à l'époque de la découverte. Dès 1819, Monsieur Raynard, professeur à l'école vétérinaire de Lyon, publia une étude sur l'un de ces mors; il avait alors "testé" le matériel archéologique sur des chevaux modernes "de taille moyenne"! (Raynard 1819 : 193). L'article était accompagné d'une lithographie représentant un des mors de Verna et une reconstitution du harnachement. La lithographie, signée Ch. Lefèvre à Lyon, représente, outre le mors, un bouton en bronze orné de croisillons, manifestement un cabochon émaillé (fig. 6 et fig. 3, objet B). La combinaison d'un mors de bride avec un mors de filet dans la bouche du cheval (ensemble : bride ou embouchure double) était traditionnellement utilisée dans la cavalerie militaire des temps modernes (fig. $\mathbf{7 3} \mathbf{~ b}$ ). Cette combinaison permet d'avoir un contact plus ferme sur la bouche du cheval, de contrôler plus facilement le port de sa tête et de son encolure et donc de le diriger avec beaucoup plus de précision. L'embouchure double est aujourd'hui utilisée par des cavaliers expérimentés sur des chevaux dressés (Muir $2000: 42$; Frey $1984: 119)$. 
113

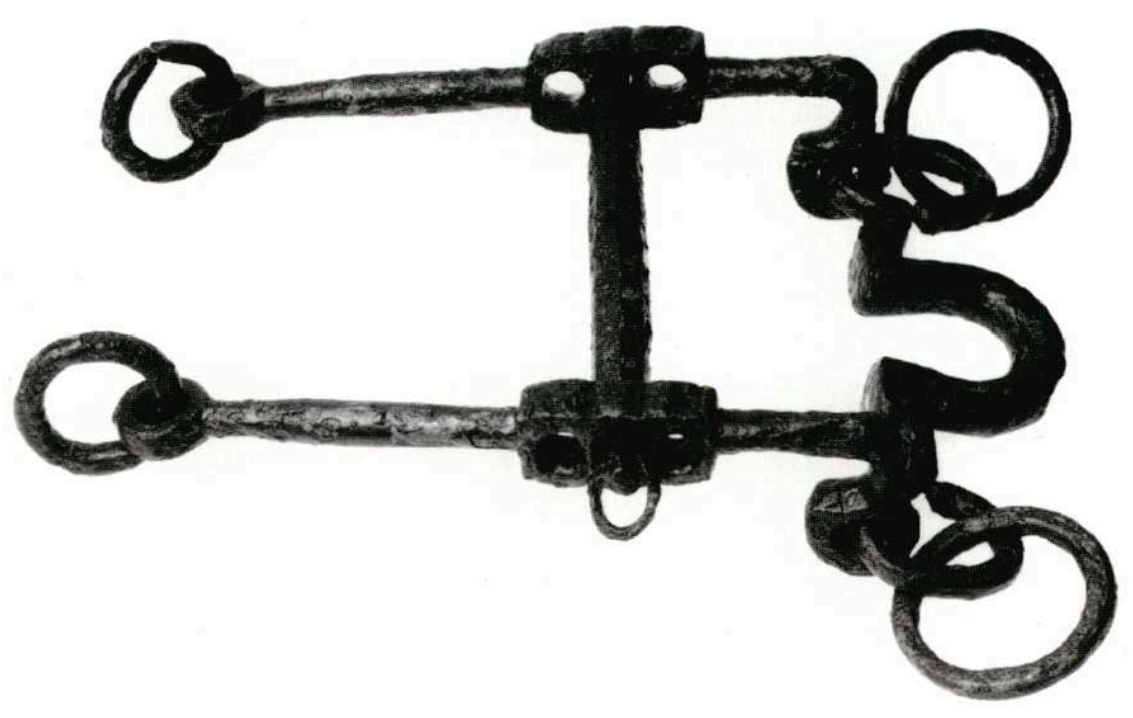

71-Mors de bride, Verna

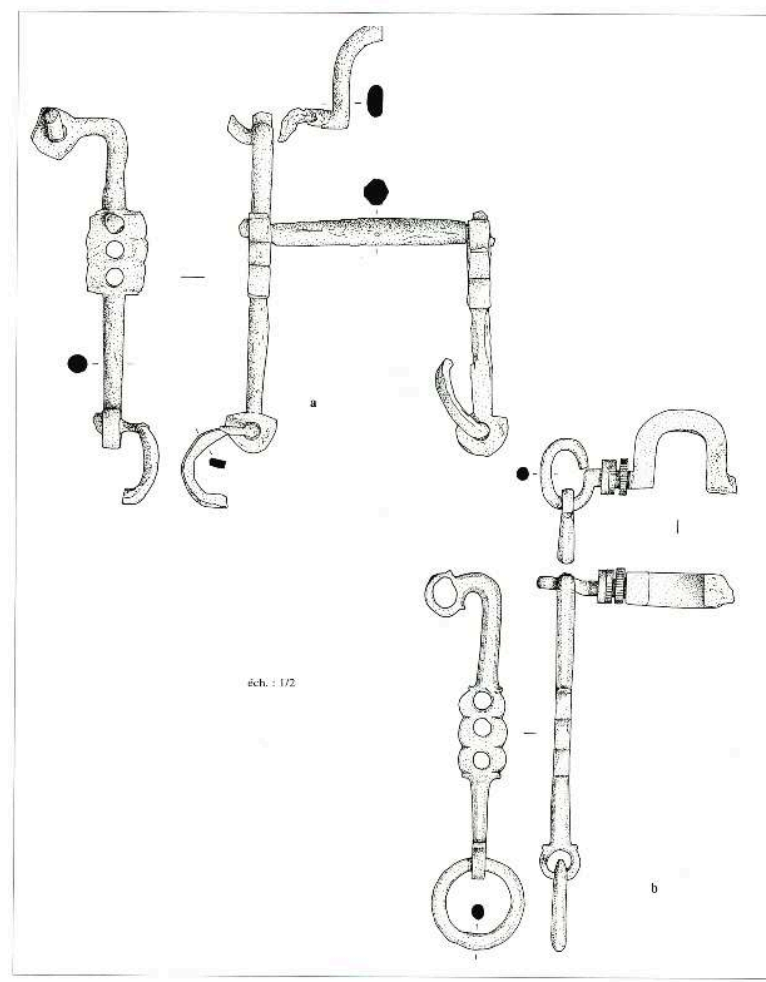

72 a et b - Mors de bride (fer) 


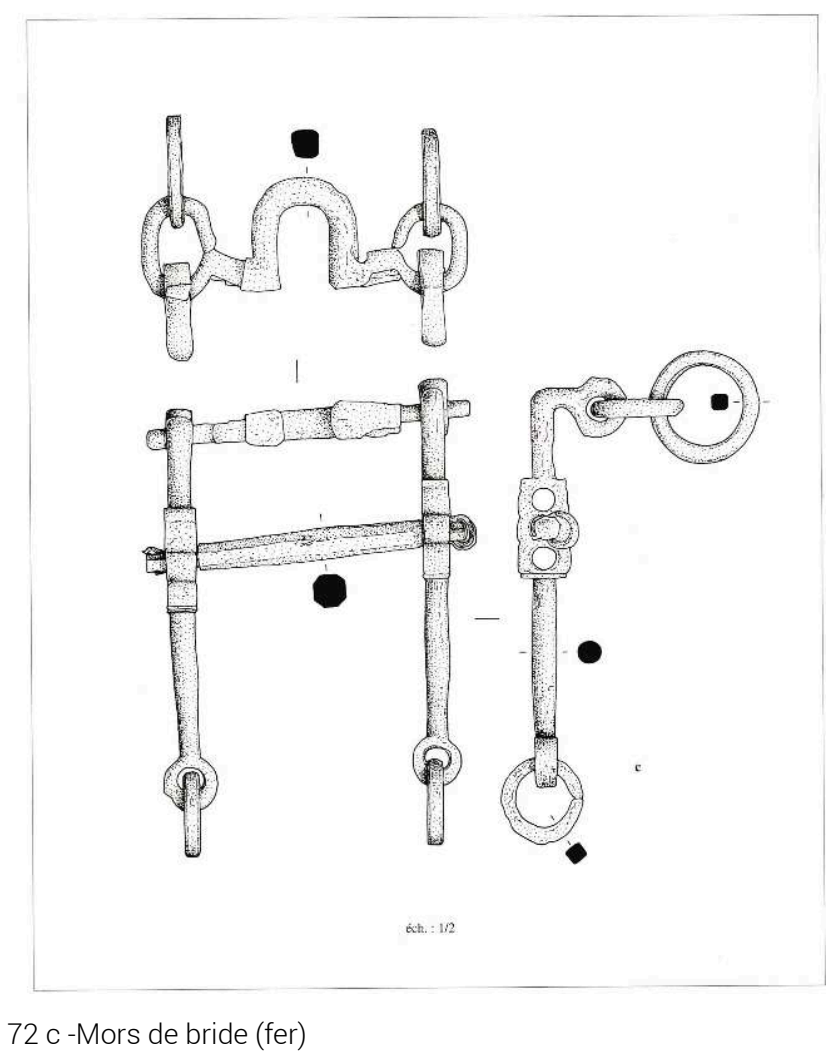

Des mors de bride ont été découverts dans des tombes à armes celtiques en Italie, comme à Canosa di Púglia (Prov. Bari, I) et à Rocca San Casciano (Prov. Forlí, I) ${ }^{161}$, ainsi que dans les camps romains de Numantia (Prov. Soria, E) (Schulten 1929: pl. 24,13; $37,8)$ datés de La Tène $\mathrm{B}$. En Europe tempérée, les mors de bride commencent à apparaître dans certaines tombes de l'horizon des oppida à la transition La Tène C2/D1 (tab. 15), et également, mais plus rarement, dans des contextes d'habitat hors oppida $^{162}$. Les exemplaires de Verna sont très proches de ceux de Saint-Romain "Verger" (Côte-d'Or) et de Léry (Eure) (fig. 74) ${ }^{163}$. Contrairement aux mors de filet (deux éléments liés, plus les deux anneaux), les mors de bride sont d'un seul élément droit et rigide. Cette caractéristique permet de mesurer leur largeur beaucoup plus précisément. Il est indispensable que la taille de l'embouchure soit adaptée à la bouche du cheval afin d'éviter des blessures qui rendraient l'animal incontrôlable. Le réglage des mors se fait grâce à des trous placés sur les tiges latérales rattachant la gourmette, ce qui permet une adaptation pour chaque cheval; le trou central est généralement le plus utilisé. Seul un des mors de Verna (fig. 72 a) ainsi que celui de Malkowice (Pow. Pińczów, PL) présentent des réglages au plus proche de l'auge du cheval (Jamka 1939-45, fig. 9 ; Baranowski 1973, fig. 30).

La largeur du mors reste constante, environ $10 \mathrm{~cm}$ pour tous les exemplaires de La Tène $B$ jusqu'à la Tène $D$ (tab. 15). Les trois mors de Verna sont de taille semblable, ce qui implique que le troisième cheval, le cheval monté, aurait été de même taille. Les progrès de l'élevage pourraient expliquer l'augmentation de la largeur du mors et de la hauteur au garrot à l'époque laténienne, mais la présence de quelques chevaux plus grands dans les oppida est aussi interprétée comme une influence romaine (Boesneck et 
al. 1971 : 30-31 ; Méniel 1987 : 36-37). Ainsi il est délicat de considérer que les mors de bride confirment cette évolution.

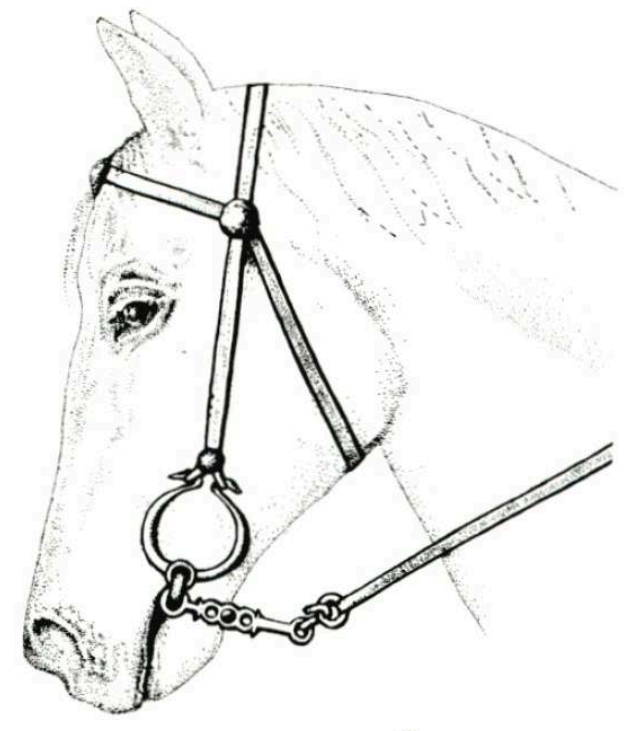

$\mathbf{a}$

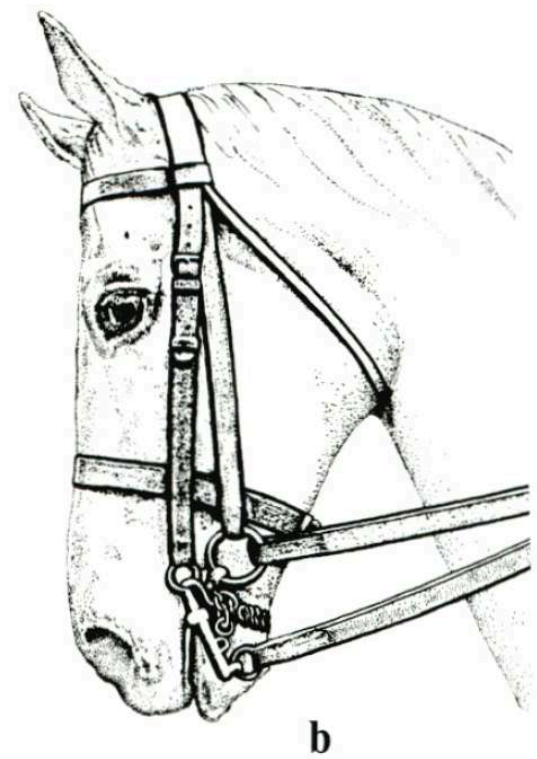

b

73 a -Embouchure antique ; b : embouchure double d'époque moderne, d'après Frey 1984 :125 fig. 4

Outre les mors de brides, existe un anneau en fer de $10,5 \mathrm{~cm}$ de diamètre interne et d'une section de $0,8 \mathrm{~cm}$, fermé par un emboîtement riveté (fig. 75). Les exemplaires de comparaison avec cet anneau sont en bronze (tab. 16), et ont deux systèmes de fermeture différents: soit, un emboîtement riveté comme celui de Verna, soit, un assemblage par demi ou à un ou deux rivets.

Ces anneaux sont souvent retrouvés en contexte d'habitats (Feugère 1994 : 148). Sans réelles possibilités d'identification, on les assimilait autrefois à des torques (van Endert 1991 : 3 ; Feugère 1994 : 148), quasi inconnus à La Tène finale. De plus, le façonnement simple de l'objet ne correspond pas aux traditions laténiennes. G. Ulbert présente des anneaux de Câceres (Prov. Extremadura, E) semblables, fermés par un assemblage par demi ou à deux rivets, et leur attribue la fonction de porte-strigiles (Ulbert $1984: 71$, pl. $12,72-74)$; toutefois, quand des strigiles sont retrouvés en contexte avec leurs anneaux, ils sont décorés de têtes de canard et présentent d'autres types de fermetures ${ }^{164}$. 


\begin{tabular}{|l|c|c|c|}
\hline \multicolumn{1}{|c|}{ Lieu de découverte } & Largeur & Datation & Bibliographie \\
\hline Verna/1 (Dép. Isère, F) & $9,9 \mathrm{~cm}$ & Lt D1 & \\
\hline Verna/2 (Dép. Isère, F) & $10 \mathrm{~cm}$ & Lt D1 & \\
\hline Verna/3 (Dép. Isère, F) & $10 \mathrm{~cm}$ & Lt D1 & \\
\hline Numantia (Prov. Soria, E) & $10,8 \mathrm{~cm}$ & Lt D1 & Schulten 1929, pl. 37,8. \\
\hline Malkowice (Pow. Pinćzów, PL) & $10 \mathrm{~cm}$ & Lt D & Baranowski 1973, fig. 30. \\
\hline Sanzeno (Prov. Trento, I) & $12,6 \mathrm{~cm}$ & $?$ & Nothdurfter 1979, pl. 32, 410. \\
\hline La Mailleraye-sur-Seine (Dép. Seine-Maritime, F) & $10 \mathrm{~cm}$ & Lt C2/D1 & Lequoy 1993: 130 fig. 12, 28. \\
\hline Roje pri Moravče, No. 1 (SLO) & $10,2 \mathrm{~cm}$ & Lt C2/D1 & Knez 1977: 121, pl. 10,3. \\
\hline Borgo San Siro (Prov. Pavia, I) & $10,8 \mathrm{~cm}$ & Lt B/C & Tizzoni 1984, pl. 89 a. \\
\hline Váglio Basilicata “ Rossano” (Prov. Potenza, I) & $10 \mathrm{~cm}$ & Lt B2 & Bottini (dir.) 1993: 229 fig. 1. \\
\hline Canosa di Púglia (Prov. Bari, I) & $10,7 \mathrm{~cm}$ & Lt B1b & Frey 1984: 124 fig. 3. \\
\hline
\end{tabular}

Tableau 15 -Les largeurs d'un ensemble de mors de bride de l'époque de La Tène (d'après bibliographie et figures)

\begin{tabular}{|c|c|c|c|}
\hline Lieu de découverte & Description & Contexte & Bibliographie \\
\hline \multicolumn{4}{|l|}{ Anneaux à emboîtement riveté } \\
\hline Verna (Dép. Isère, F) & $\begin{array}{l}\text { fer, dia. } 10,5 \mathrm{~cm} \text {, } \\
\text { section } 0,8 \mathrm{~cm}\end{array}$ & $\begin{array}{l}\text { tombe à char riche avec } 3 \\
\text { mors de bride italiques }\end{array}$ & \\
\hline $\begin{array}{l}\text { Manching } \\
\text { (Lkr. Pfaffenhofen a. d. Ilm, D) }\end{array}$ & $\begin{array}{l}\text { bronze, } \\
\text { dia. environ } 13 \mathrm{~cm} \text {, } \\
\text { section } 0,5-0,8 \mathrm{~cm}\end{array}$ & $\begin{array}{l}\text { oppidum, plusieurs exemplaires, } \\
\text { provenant en partie du dépôt } \\
\text { de Leisenhardfeld }\end{array}$ & Van Endert $1991: 3$ pl. 1, 1-9. \\
\hline Belgrad/Karaburma tombe $16(\mathrm{YU})$ & $\begin{array}{l}\text { bronze, } \\
\text { dia. } 12,0 \mathrm{~cm} \text { interne } \\
\text { (légèrement écarté) }\end{array}$ & tombe avec 1 mors de bride thrace & $\begin{array}{l}\text { Todorović } \\
\text { Karaburma pl. } 6 .\end{array}$ \\
\hline Zemun/Gardoš (YU) & bronze, écarté & trouvaille sur un cimetière, avec un éperon & Božič $1984: 135$ fig. 1-2. \\
\hline Kralevo/Tărgovište (BG) & bronze, dia. $9 \mathrm{~cm}$ & $\begin{array}{l}\text { comblement d'un tertre avec un mors à } \\
\text { anneaux pointus et à tiges latérales à deux } \\
\text { trous. (dia. interne environ } 12 \mathrm{~cm} \text { ) }\end{array}$ & Kull 1996 : 429 fig. $8,2$. \\
\hline Mezek/Haskovo "Maltepe" (BG) & $\begin{array}{l}\text { bronze, } 3 \text { exemplaires, } \\
\text { dia. environ } 9 \mathrm{~cm} \text {. }\end{array}$ & tombe à char riche, Lt B2 & Kull 1996 : 429 fig. $8,3$. \\
\hline \multicolumn{4}{|c|}{ Anneaux à assemblage par demi et 1 ou 2 rivets } \\
\hline Numantia (Prov. Soria, E) & $\begin{array}{l}\text { fer, } 2 \text { rivets, } \\
\text { dia. } 11-12 \mathrm{~cm}\end{array}$ & camp romain, " Renieblas" & Schulten $1929: 208, \mathrm{pl} .23,11$. \\
\hline $\begin{array}{l}\text { Cáceres el Viejo } \\
\text { (Prov. Extremadura, E) }\end{array}$ & $\begin{array}{l}\text { bronze, } 3 \text { exemplaires } \\
\text { (dont } 2 \text { à } 2 \text { rivets et } \\
1 \text { fragment) }\end{array}$ & $\begin{array}{l}\text { camp romain, terminus ante quem } 80 / 77 \\
\text { av. J.-C. }\end{array}$ & Ulbert 1984, pl. 12, 72-74. \\
\hline Besançon (Dép. Doubs, F) & $\begin{array}{l}\text { bronze, fragment, } \\
1 \text { rivet }\end{array}$ & $\begin{array}{l}\text { oppidum, Parking de la Mairie } \\
\text { "Maison du chef" }\end{array}$ & Feugère $1994: 148$ - 153 fig. 4,1 \\
\hline Ensérune (Dép. Hérault, F) & bronze, fragment & habitat (?) & Feugère 1994 : 148 - 153 fig. $4,2$. \\
\hline $\begin{array}{l}\text { Mas d'Agenais } \\
\text { (Dép. Lot-et-Garonne, F) }\end{array}$ & $\begin{array}{l}\text { bronze, fragment, } \\
1 \text { rivet }\end{array}$ & habitat & $\begin{array}{l}\text { Feugère } 1994 \text { : } \\
148-153 \text { fig. } 4,3 \text {. }\end{array}$ \\
\hline
\end{tabular}

Tableau 16 -Anneaux à fermeture rivetée

219 Les études de B. Kull sur quelques tombes de l'Europe de l'Est indiquent que ces anneaux sont trouvés dans les bouches mêmes des chevaux ${ }^{165}$. Aujourd'hui, des anneaux semblables sont utilisés pour les mors de filets de présentation en main : ces mors dits "Tattersall" servent également pour les jeunes chevaux rétifs (Muir $2000: 47$ ). Ils sont composés d'un anneau placé dans la bouche, la têtière fixée par deux anneaux latéraux placés sur l'anneau et la longe s'attachant à la base.

Pour La Tène, l'interprétation comme mors de filets de présentation à la main est aussi possible. A Verna, on peut envisager un scénario suivant lequel le cheval de guerre du défunt aurait été exhibé, puis l'on aurait déposé son mors de bride dans la tombe. 
Trois mors annulaires en bronze (Kull 1996: 429-430, fig. 8, 3) ont été trouvés dans la riche tombe à char de Mezek (BG) (Filow 1937 ; Fol 1991). Dans ce cas, les chevaux ont pu être conduits à la main par des garçons d'écurie, et non par le conducteur du char, le troisième mors étant pour le cheval monté. Ces objets en fer de Mezek étant inédits, l'interprétation avancée reste incertaine.

Si l'on envisage une autre hypothèse, il semble possible d'utiliser le mors annulaire et le mors de bride ensemble, comme double embouchure. Aujourd'hui, les mors de bride sont complétés par des mors de filet (fig. $\mathbf{7 3}$ b). Cette combinaison permet d'avoir un contrôle plus précis du cheval, approprié au terrain et aux besoins du cavalier armé. Le mors annulaire a une action moindre sur les barres et la langue du cheval qu'un mors de filet, le cavalier le contrôlant plus difficilement. Il est tout de même possible de diriger un cheval sans mors, uniquement avec un licol. La combinaison mors de bride/ mors de filet est une embouchure considérée aujourd'hui comme très dure et blessante; mais le mors annulaire et le mors de bride ensemble comme double embouchure pouvait être possible à l'époque.

Du point de vue archéologique, il est peu probable que le cheval du cavalier (ou le statut du cavalier) soit montré deux fois séparément, par le mors de bride et par le mors annulaire. Pour Verna l'argumentation demeure hypothétique, l'association anneau et mors de bride étant déjà une interprétation à partir d'une collection ancienne. A contrario, un mors de bride et un mors annulaire font partie de la tombe 16 de Belgrade/Karaburma (YU) (Todorovic 1972, pl. 6). La tombe de Gela "Priolo" (Prov. Caltanissetta, I) est un autre exemple de cette combinaison: seules les deux tiges latérales d'un mors de filet étrusque et l'anneau en bronze sont publiés dans ce cas ${ }^{166}$.

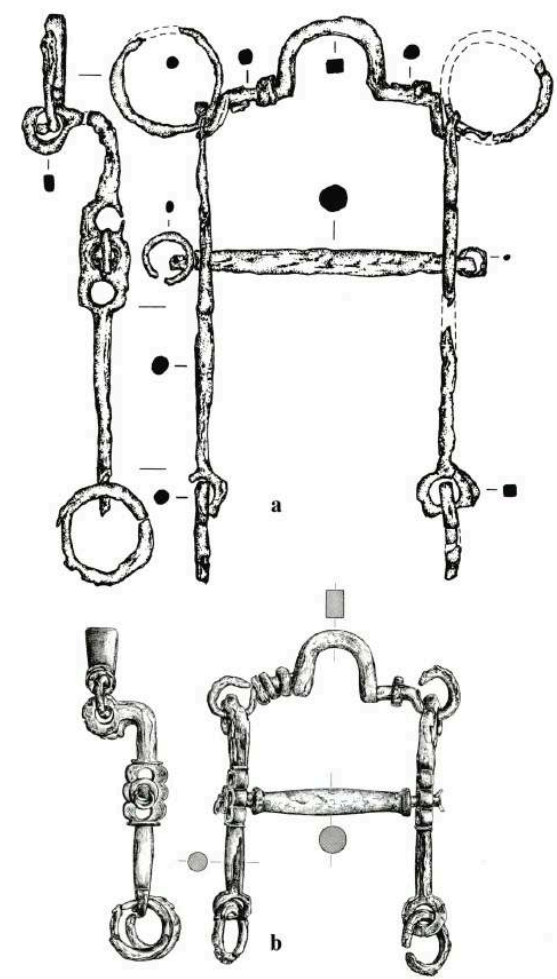

74 a -Saint Romain "Verger" (Côte-d'Or) ; b : Léry (Eure) d'après Bourgogne médiévale1987 :177 nº 438 ;Normandie 1990 : 51. 


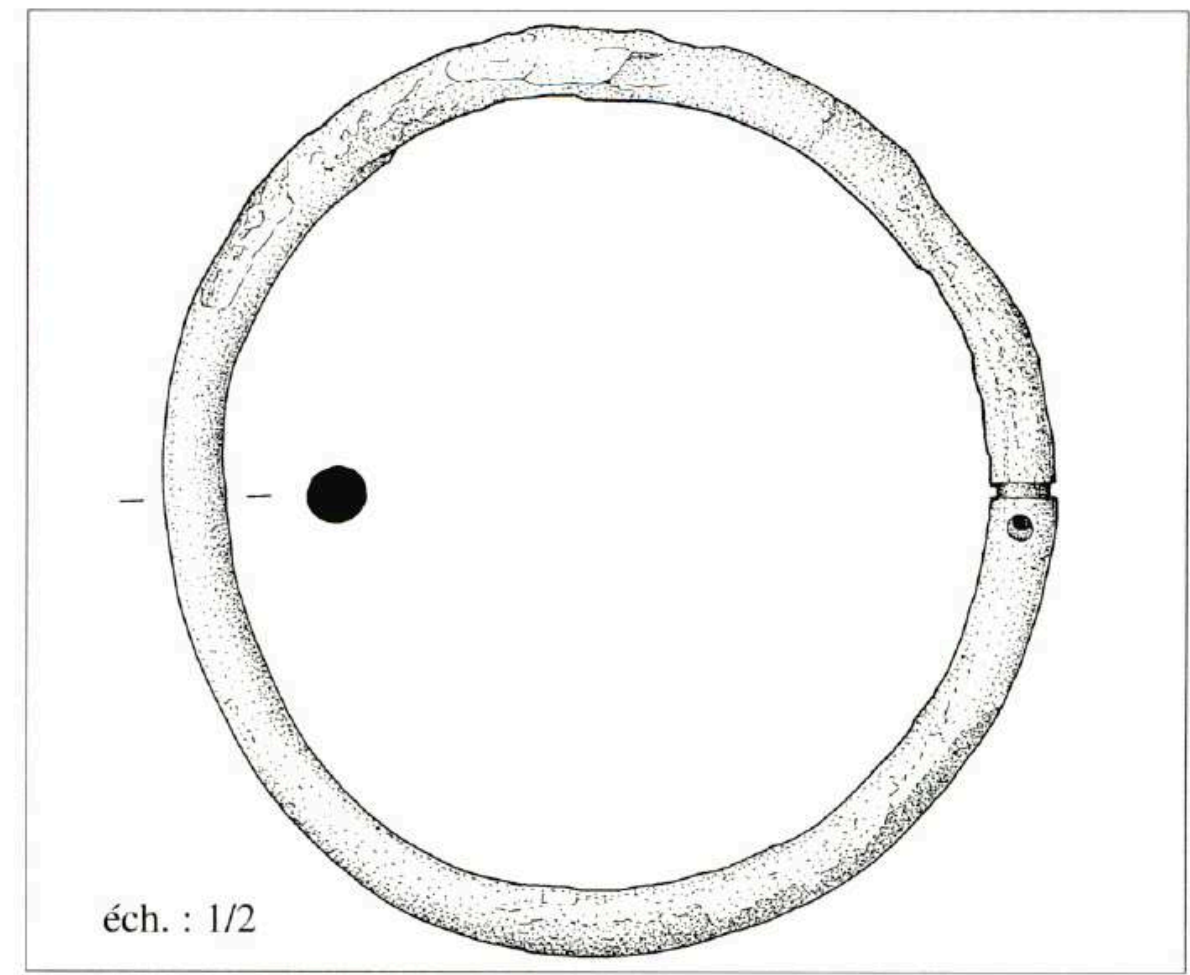

75-Mors annulaire (fer)

\begin{tabular}{|c|c|c|c|c|}
\hline Lieu de découverte & $\begin{array}{l}\text { Equipement } \\
\text { du cavalier }\end{array}$ & D'autres objets d'accompagnement & Datation & Bibliographie \\
\hline \multicolumn{5}{|c|}{ Mors } \\
\hline Adria (Prov, Rovigo, I) & mors de bride & $\begin{array}{l}2 \text { mors de filet avec les chevaux du } \\
\text { char, cheval du cavalier, char }\end{array}$ & LCC & Trey 1976. \\
\hline Léry (Dép. Eurc. F) & mors de brite & $\begin{array}{l}\text { ensemble funéraire avece êpéc, } 2 \text { mors } \\
\text { de filel, ceaux, outili, bracelel }\end{array}$ & $\angle C D$ & Normandie 1990. \\
\hline $\begin{array}{l}\text { La Mailleraye sur Scine } \\
\text { (Dép. Seine-Maritime, F) }\end{array}$ & mors de bride & $\begin{array}{l}\text { sepulture multiple avec } 3 \text { nors de filct, } \\
\text { clements de char, armes, ustensiles } \\
\text { de cuisine }\end{array}$ & $4 \mathrm{CDI}$ & Treyway 1999. \\
\hline $\begin{array}{l}\text { Bad Nauhteim "Fund 60" } \\
\text { (Wenteraukreis, D) }\end{array}$ & mors de filict & $\begin{array}{l}2 \text { vases cn terre cuitt, tallon de lance, } \\
\text { perle (?) }\end{array}$ & $\angle C O D$ & $\begin{array}{l}\text { Schöntberger 1952, } \\
\text { pl. 8. 5-9. }\end{array}$ \\
\hline Verna (Dép. Lietre, F) & mors de bride & 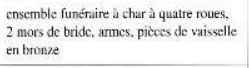 & LIDI & \\
\hline $\begin{array}{l}\text { Mayen "Amtsgecicht" } \\
\text { (Kr. Mayen-Koblenz, D) }\end{array}$ & mars de filet & trouvaille sur un cimetiere & LD & $\begin{array}{l}\text { Oesterwind 1996: } \\
\text { 9s fig. 10, I. }\end{array}$ \\
\hline $\begin{array}{l}\text { Hofhcim, tumbe } 8 \\
\text { (Main - Taunus-Kreis, D) }\end{array}$ & $\begin{array}{l}\text { mars de type } \\
\text { Hofhcim }\end{array}$ & poignard, scaux, hachc, ceramiume & LD & Wener 1953. \\
\hline $\begin{array}{l}\text { Titelberyff.amadeleine, } \\
\text { tombx } 3 \text { (L) }\end{array}$ & mors de filet & $\begin{array}{l}\text { epoce, lance, bouclier, fitules, truteau, } \\
\text { dé (...) }\end{array}$ & LI D2a & $\begin{array}{l}\text { Metzer Zens e e al. } \\
\text { 1999. }\end{array}$ \\
\hline $\begin{array}{l}\text { Heinbuch-Weis, tombe } 2 \\
\text { (Kr. Neuwichl, D) }\end{array}$ & mors de filst & $\begin{array}{l}2 \text { mors de filet, éléments de char, armes, } \\
\text { ustensiles de cuisine }\end{array}$ & Lt D2a & Joachim 1973. \\
\hline \multicolumn{5}{|c|}{ Eperons } \\
\hline $\begin{array}{l}\text { Nicder Olm } \\
\text { (Kr. Mairr-Bingen, D) }\end{array}$ & épering & trouvaille sur un cimetic̀re & $\mathrm{L} . \mathrm{C} 2 \mathrm{D}$ & $\begin{array}{l}\text { Stünpel 1954: } \\
86 \mathrm{pl} .7,20 \text {. }\end{array}$ \\
\hline $\begin{array}{l}\text { Goetlingen-Nospett. } \\
\text { tumbes A-D (L) }\end{array}$ & $\begin{array}{l}\text { Épcrons dans toutes } \\
\text { les } 4 \text { tombes }\end{array}$ & umblhes à épée richementéquipées & 40102 & $\begin{array}{l}\text { Metier ef at. } 1991 \text { : } \\
113 \text { sqq. fig. } 86-88 .\end{array}$ \\
\hline Pétange (L) & 2 éperons & trouvaille sur un cimctière & L. D2 & $\begin{array}{l}\text { Metiler et ad. } 1991 \text { : } \\
18 \text { fig. } 5 \text {. }\end{array}$ \\
\hline $\begin{array}{l}\text { Kintr-Filisen, bombe } 1 \\
\text { (Kr. Trier-Sarrbury, D) }\end{array}$ & Epron & eprec, fihule, ciseau & Lt D2 & $\begin{array}{l}\text { Hatfner 1974a : } \\
53 \text { fig. } 1 \text {. }\end{array}$ \\
\hline $\begin{array}{l}\text { Kuntu-Fil sen, tombe } 3 \\
\text { (Kr: Trier Sartourg. D) }\end{array}$ & éperon & $\begin{array}{l}\text { lance, cicauu, } 5 \text { pitites de vaisselle } \\
\text { en terre cuite }\end{array}$ & $\mathrm{Lt} \mathrm{D}_{2}$ & $\begin{array}{l}\text { Hefffner 1974a: } \\
54 \text { sq4. fige. } 2,8-13 \text {. }\end{array}$ \\
\hline $\begin{array}{l}\text { Kollig, tombe } 6 \\
\text { (Kr.Mayen-Koblenr, D) }\end{array}$ & Eperon & $\begin{array}{l}2 \text { mors de filiet droit, phalierss, bovdlict, } \\
\text { fibule, lance (?) }\end{array}$ & $4 \mathrm{D} 2 \mathrm{~b}$ & Joachim 19794. \\
\hline 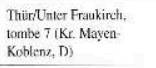 & Éperon & $\begin{array}{l}\text { boucle de ceinturr, vaiselle en terre cuite, } \\
\text { clous et țìles decoratits }\end{array}$ & L $102 \mathrm{~b}$ & $\begin{array}{l}\text { Bockius } 1990 \text { : } \\
150-151 \text {. fig. } 3,11 \text {. }\end{array}$ \\
\hline
\end{tabular}

Tableau 17 -Tombes de La Tène $C$ et $D$ avec un ou trois mors, ou avec des éperons chez les Celtes occidentaux (France, Luxembourg, Sud et Ouest de l'Allemagne, Italie du Nord)

Enfin, dans la tombe de guerrier de La Tène B1 de Landau in der Pfalz (Stadt Landau in der Pfalz, D) (Engels 1974: 54, pl. 41, 5), un autre anneau en bronze de 8,6 $\mathrm{cm}$ de 
diamètre avec un assemblage par demi et par rivet avait été interprété comme un bracelet. Dans ce cas, il est difficile de savoir s'il s'agit d'un mors réutilisé ou d'un type particulier de bracelet.

Aujourd'hui, les tombes dans lesquelles ont été déposés des éperons ou des mors seuls sont interprétées comme des tombes de cavaliers ${ }^{167}$. Si elles ne livrent pas toujours des objets qui permettent de déterminer le sexe de la personne enterrée, les armes indiquent qu'il s'agit d'hommes dans la plupart des cas (tab. 17). Aucune tombe contenant des parures féminines n'a pu à ce jour être interprétée comme celle de cavalière, ce que confirment les rares analyses d'ossements.

notion de cavalier n'existe pas dans les traditions funéraires de La Tène $B$ et $C$ en Europe tempérée. Par contre, les sources historiques parlent de cavaliers à l'époque des migrations celtiques (Dobesch 1996). Un cheval décapité fait également partie du charnier des guerriers de La Tène $\mathrm{C} 1$ de Ribemont-sur-Ancre (dép. Oise) (Brunaux dir. 1999 : 203). Sur ce site, la présence de cavaliers est attestée, mais en général, elle n'est pas représentée par des objets dans les tombes.

Dans la tombe 6 de Kollig (Kr. Mayen-Koblenz, D) (Joachim 1974), les deux mors de filet droit sont des objets pars pro toto qui signalent le char; de plus un éperon indique la présence du cavalier. Ainsi, un troisième mors associé à un éperon de cavalier ne doit pas être considéré comme celui d'un troisième cheval d'attelage, mais bien celui d'un cheval monté. Un troisième mors - en plus de la paire des chevaux de l'attelage - a été retrouvé dans plusieurs tombes à char de La Tène $\mathrm{D}^{168}$. Le char et les trois chevaux du cimetière d'Adria (Prov. Rovigo, I) reste le meilleur exemple : deux mors de filet pour les chevaux attelés et le mors de bride pour le cheval monté étaient en place (Frey 1976 : 173-174). De même, un mors de bride italique dans le dépôt funéraire à plusieurs chars de La Mailleraye-sur-Seine (dép. Seine-Maritime) indique qu'un des guerriers était aussi considéré comme un cavalier (Lequoy 1993 : 130 fig. 12, 28). Le mors de bride est, dans ce cas, une importation tout comme les deux pièces de vaisselle en verre. Autre exemple, le complexe funéraire de Léry (dép. Eure) (Normandie $1990: 50-53$ ) : une paire de mors de filet représenterait comme pars pro toto le conducteur du char, alors que le mors de bride indiquerait que le guerrier à l'épée était en même temps un cavalier. Cette image est plausible, mais hypothétique, l'ensemble n'étant pas clos. Citons aussi le guerrier de la tombe à char de Heimbach-Weis (Kr. Neuwied, D) qui a été enterré avec un mors de filet et deux éperons différents (Joachim 1973: 33 ; fig. 11, $9-10 ; 12,14)$ ce qui prouve qu'il était cavalier, mais aussi conducteur de char. Dans la tombe à char à quatre roues de Cugir (RO) (Crisan 1993, pl. 11), les mors de bride sont aussi au nombre de trois. La présence d'éperons ou de mors simples ou triples dans les tombes n'est pas forcément représentative d'une couche sociale uniforme des "equites" ou des "cavaliers" 169 . Ces objets ne correspondent pas aux insignes du statut social des “cavaliers", les tombes concernées étant trop variées. De plus, César utilise la terminologie romaine pour désigner les classes sociales celtiques, ce qui n'a aucun rapport avec l'équipement funéraire.

L'éperon et le mors de filet représentant le cavalier n'attestent aucun caractère social suprarégional. Par contre, leur forte présence dans les tombes de la région RhinMoselle (Joachim 1974 : 163) atteste un rite funéraire local, dans lequel sont inclus les conducteurs de chars. Quelques tombes comme celles de Goeblinge-Nospelt (L) ${ }^{170}$ et de Heimbach-Weis (Joachim 1973) sont très riches par rapport à celles de Konz-Filsen (Kr. Trier-Saarburg, D), présentées par H.-E. Joachim (Joachim 1974 : 166). On remarque la 
concentration des tombes de cavaliers dans la région des Trévires, cette forte présence de la cavalerie étant par ailleurs mentionnée dans les sources écrites ${ }^{171}$. H.-E. Joachim ${ }^{172}$ évoque la possibilité que les tombes à char soient devenues, par la suite, des tombes de cavaliers. Le rite funéraire du dépôt d'éperons et de mors se retrouve dans une autre région: la Yougoslavie ${ }^{173}$. Une forte concentration d'éperons est également connue autour d'Hambourg en Basse-Elbe ${ }^{174}$. Ces rites funéraires locaux doivent être interprétés différemment du reste de l'Europe, les cavaliers appartenant à des tombes à char riches. Dans les régions où leur répartition est très dense, les mors et les éperons ne sont plus des marqueurs sociaux comme dans les tombes de Verna, La Maillerayesur-Seine ou Léry, mais contribuent à la richesse funéraire de ces tombes. Les mors de bride attestent d'une importation ou d'une origine étrangère des objets.

\section{L'OUTILLAGE. J.-P. Guillaumet, M. Schönfelder}

Un unique objet a été clairement identifié comme outil dans l'ensemble du mobilier.

Il s'agit d'une faucille en fer (L. total $33 \mathrm{~cm}$ ), à la lame large de $28,5 \mathrm{~mm}$ d'ouverture et d'un poids de $354 \mathrm{~g}$ (fig. 76). La fixation du manche en bois se fait à l'extrémité de la partie coupante (ou lame), au moyen d'une virole fixée sur le fer par un rivet et par un bouton enfoncé dans le bois. Ce système de fixation du manche est attesté sur une faucille du dépôt gaulois de Larina (Perrin 1990 : 74-75) et en Dacie entre le IIe s. av. J.C. et la fin du Ier s. ap. J.-C. (Glodariu, Iaroslavschi 1979 : fig. 33). Ce type de manche avec virole se retrouve dans de nombreux sites de Bohème, Moravie et Slovaquie, comme celui de Kolín (CZ) (Rybová, Motyková 1983 : pl. 12) daté de la fin de La Tène.

La présence d'outils est connue dans d'autres tombes aristocratiques des Bituriges et des Trévires (Ferdière, Villard : 209 sq., 275, 280-285 ; Metzler et al. 1991 : 64, 103, 172). Ces outils sont considérés par nos collègues, non comme les signes d'une activité artisanale ou paysanne, mais comme les symboles d'une responsabilité de la gestion de ressources minières ou agricoles. Ils sont aussi le fondement de la prospérité de la famille et de la tribu du défunt.

\section{LES OBJETS MÉTALLIQUES DIVERS. M. Schönfelder}

Une partie du mobilier de la collection de Verna ne peut trouver de fonction précise. L'état de conservation de certains objets est néamoins identique aux autres objets de La Tène finale ce qui permet de les inclure dans cet ensemble funéraire.

\section{Tôles en bronze profilées}

Plusieurs fragments de tôles en bronze en forme de bande (larg. 3,4-4,0 cm ; long. max. conservée $13,5 \mathrm{~cm}$ ) devaient être fixés à l'aide de petits clous - seuls les trous en témoignent - sur un élément de bois (fig. 77). S'il a parfois été possible d'associer certaines tôles décorées avec le char, cela demeure impossible pour ces dernières en bronze profilées. Peut-être s'agit-il d'éléments de meubles, par exemple, mais nos connaissances sur le mobilier de La Tène autre que funéraire sont trop limitées. 


\section{Attache ajourée avec bouton émaillé}

L'attache en bronze (fig. 78 et fig. 3, objet D) ressemble à une agrafe de ceinture à bouton émaillé, composé d'une lyre à branches en forme de $\mathrm{S}$ de section triangulaire. $\mathrm{A}$ la base, il était fixé par un piton à œil sur une autre objet (en bois ?) inconnu.

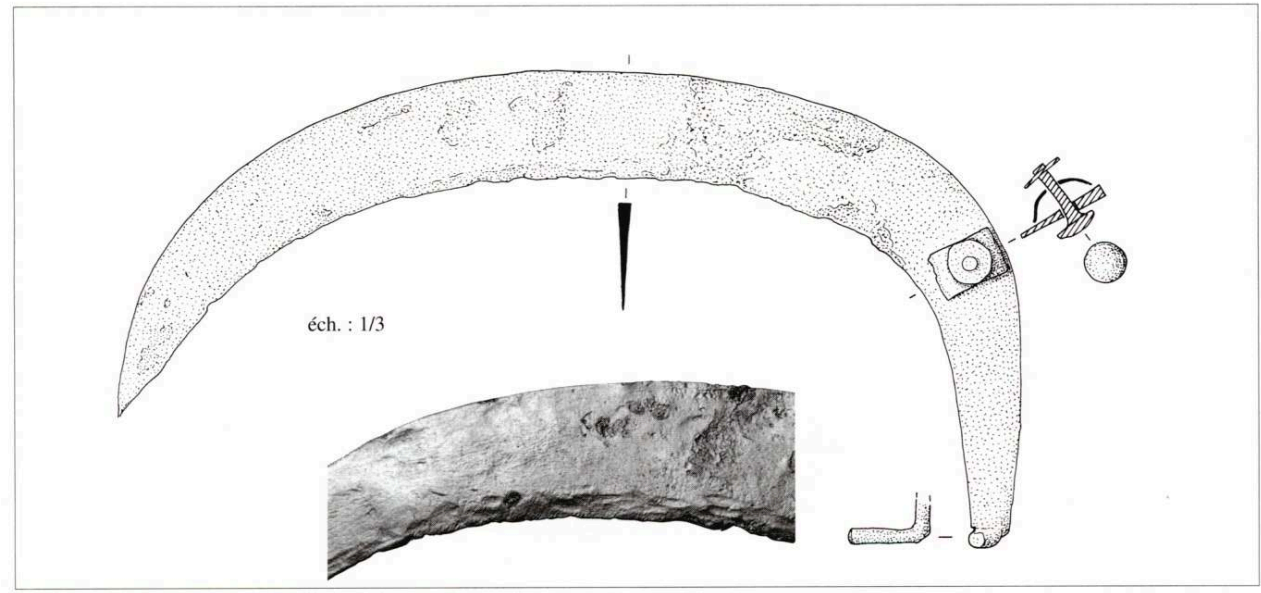

76-Faucille et détail de la surface
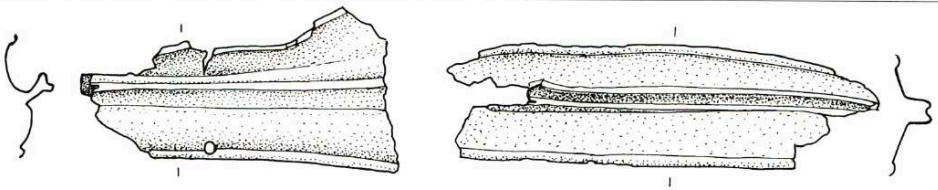

éch. : 1/2
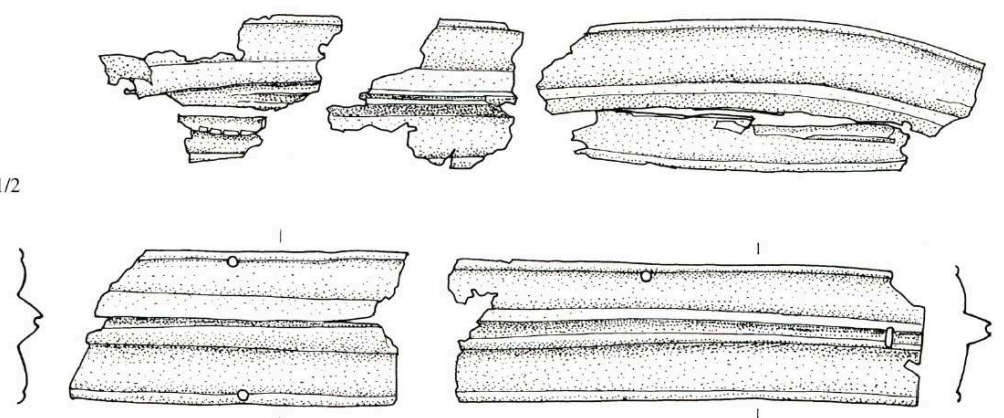

77-Fragments de tôles (bronze)

\section{3. “Nœud papillon” en bronze}

Un petit nœud papillon en tôle de bronze (long. 7,0 cm, larg. 2,0 cm) était fixé par un petit clou en bronze. La surface porte des traces de feu (fig. 79). 
éch. : $1 / 2$

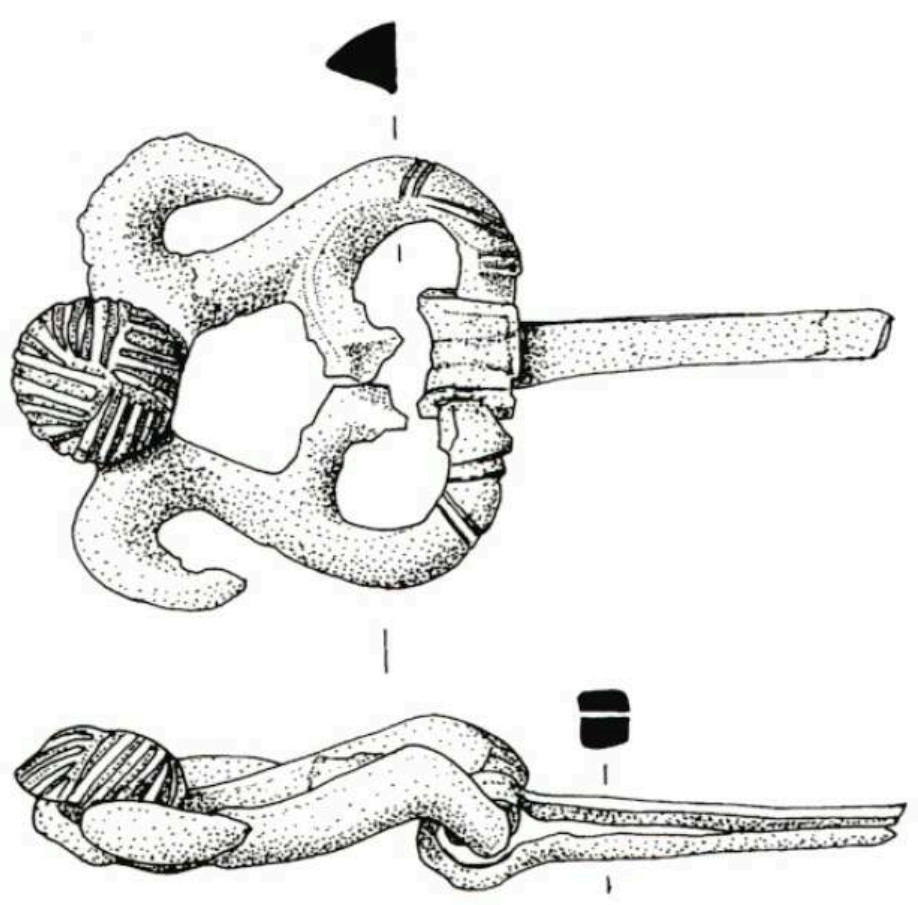

78-Attache (bronze)

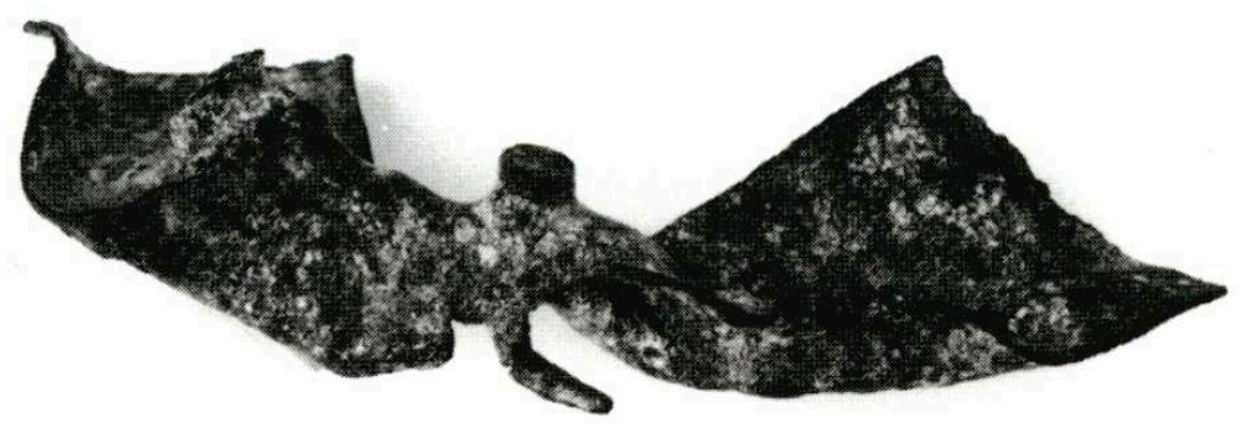

79-Noeud papillon en tôle (bronze)

\section{Anneaux en bronze}

On constate huit fragments d'anneaux en bronze de diamètres et de sections variables (fig. 80) ; quelques-uns portent des traces de feu. Un neuvième fragment est torsadé. 
123
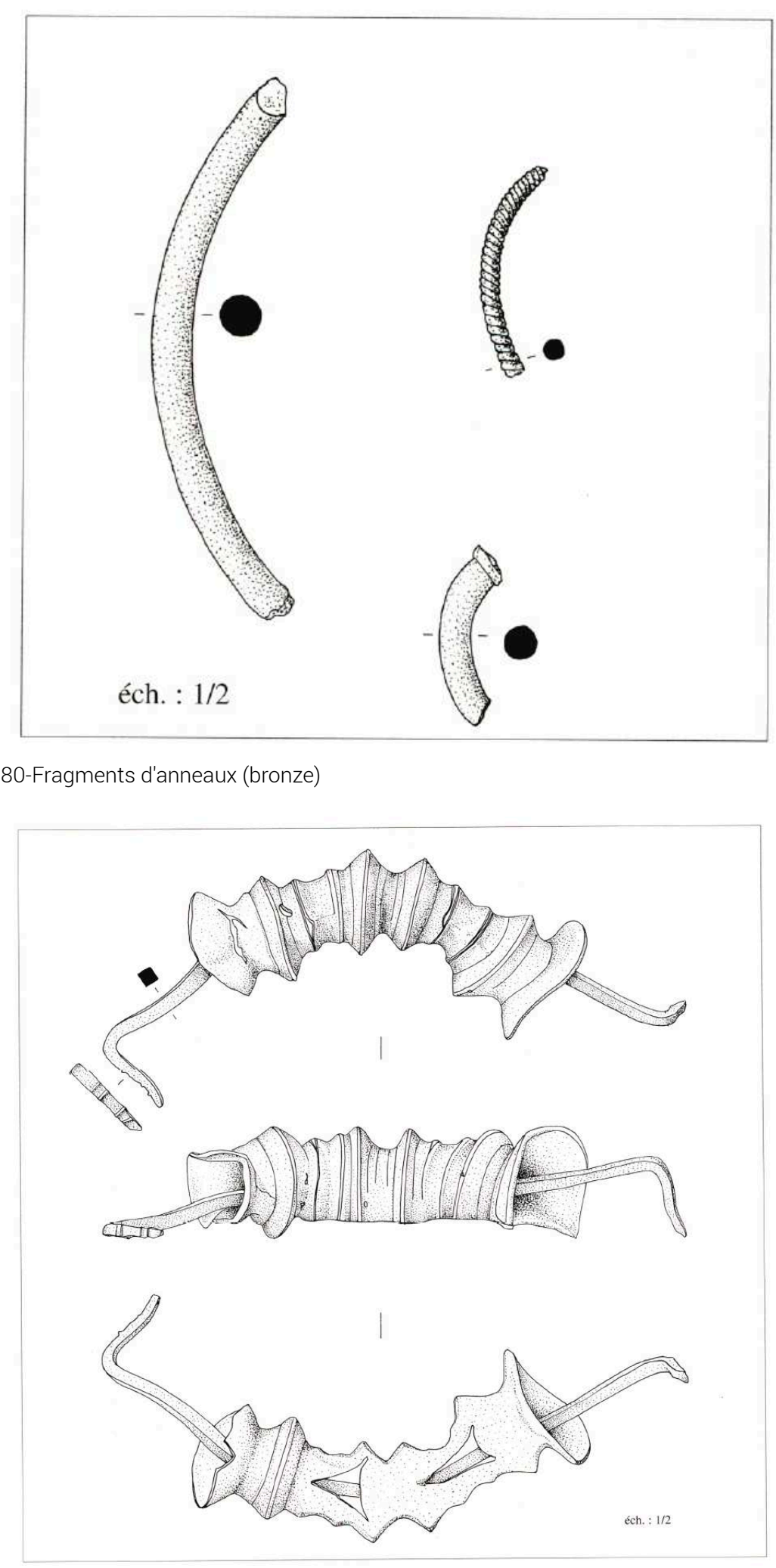

81-Elément de décoration (bronze) 


\section{Eléments massifs de décoration en bronze sur fer}

Deux éléments massifs de décoration sont singuliers et aucune fonction définitive ne peut leur être attribuée. Ce sont des tiges en fer de section carrée, courbées, portant un décor en bronze coulé. L'une des deux pièces, qui porte des traces fortes de feu (fig. 81) se termine par des crochets, l'un vers le bas, l'autre vers l'intérieur (long. max. $29,0 / 27,4 \mathrm{~cm}$ ) alors que l'autre tige est symétrique. Par ces crochets les objets devaient être fixés dans un élément en bois massif. Les décors en bronze coulé ont la forme des tuyaux courbés et profilés (dia. 5,1 cm). Ils sont aplatis au milieu sur la base; ces éléments étaient donc plaqués sur le bois sur lequel ils étaient fixés par les crochets. Ces décors peuvent être interprétés comme des éléments du char, situés peut-être dans les angles de la caisse, bien qu'aucun parallèle n'existe et que leur nombre ne témoigne pas en faveur de cette hypothèse.

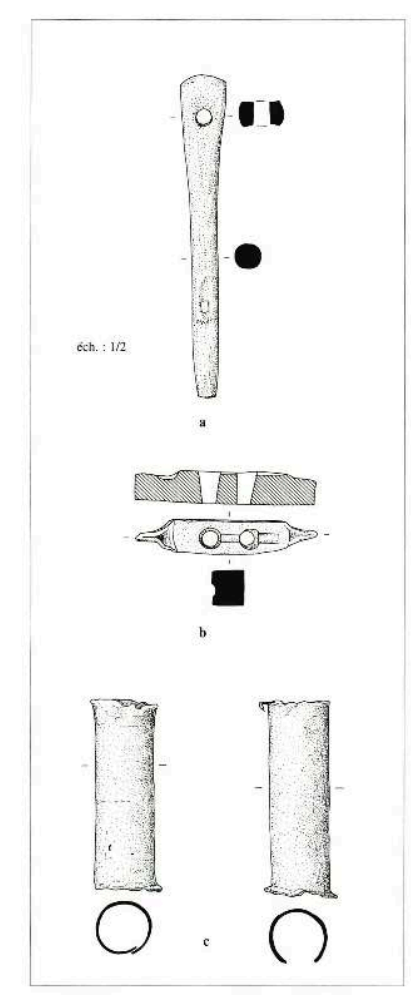

$82 \mathrm{a}$-Tige ; $\mathrm{b}$ : objet à deux trous ; $\mathrm{c}$ : cylindres en fer

\section{Tige à deux trous}

Une tige à deux trous (long. 13,5 cm), légèrement conique fait partie de l'ensemble (fig. 82 a). Les deux extrémités ne montrent pas de traces caractéristiques d'un outil. La section rectangulaire de l'un des trous, typique, trouve des parallèles dans l'habitat ouvert de Roanne (Loire), dans le sanctuaire de Nogent-sur-Seine (Aube) et sur l'oppidum de Manching (Lkr. Pfaffenhofen an der Ilm, D) ${ }^{175}$. Sa fonction n'est pas identifiable. 


\section{Objet en fer à deux trous}

Une tige à section rectangulaire (long. $7,4 \mathrm{~cm}$ ) présente deux trous légèrement coniques (dia. 0,5-0,7 cm) (fig. 82 b) ; les extrémités amincies servaient probablement à maintenir l'objet. L'utilisation comme filière est à exclure, au vu de la forte épaisseur du fil exigé par l'outil, incompatible avec un travail manuel. La fonction de l'objet reste donc inconnue, ce dernier n'apportant pas d'élément nouveaux au dossier très discuté du tréfilage à l'âge du Fer (Jacobi 1979).

\section{Cylindre en tôle de fer}

240 Deux cylindres en tôle de fer (dia. 2,4 cm, long. 7,9/8,1 cm) ont une fonction inconnue (fig. 82 c). Les tôles sont pliées en forme de tube non fermé. Des comparaisons existent sur l'oppidum de Manching en Bavière ${ }^{176}$.

\section{Agrafes de charpenterie}

Deux agrafes peuvent être attribuées au char grâce à leur décoration. Trois autres agrafes (fig. 83) sont plus longues et sans décor (long. max. 31,2/28,2/25,6 cm ; larg. $3,1 / 3,3 / 3,3 \mathrm{~cm}$ ) ; l'une est dessinée sur une planche de 1818 (fig. 4, objet FF). Ce sont d'ordinaire des agrafes de charpenterie. Peut-être ont-elles été utilisées pour une construction en bois avant de procéder à l'incinération, voire après, pour y recueillir les charbons et les restes du mobilier fondu.

\section{Renforcements d'angles en tôle de fer}

Cinq tôles en fer rectangulaires (environ 6-8 cm x 20-20,5 cm) ont été pliées pour renforcer des angles droits en bois (fig. 84 a). Chacune était fixée par quatre grands clous à têtes hémisphériques (long. 4,3-4,6 cm) dans les angles. Elles sont aujourd'hui comprimées, mais leurs angles d'origine sont encore visibles en deux endroits. Un de ces objets figure sur une planche ancienne (fig. 5, objet V). Ces tôles ne sont probablement pas des agrafes de jantes des roues, les clous étant trop massifs; de plus, les agrafes de jantes des autres tombes à char ne présentent pas la même forme ${ }^{177}$.

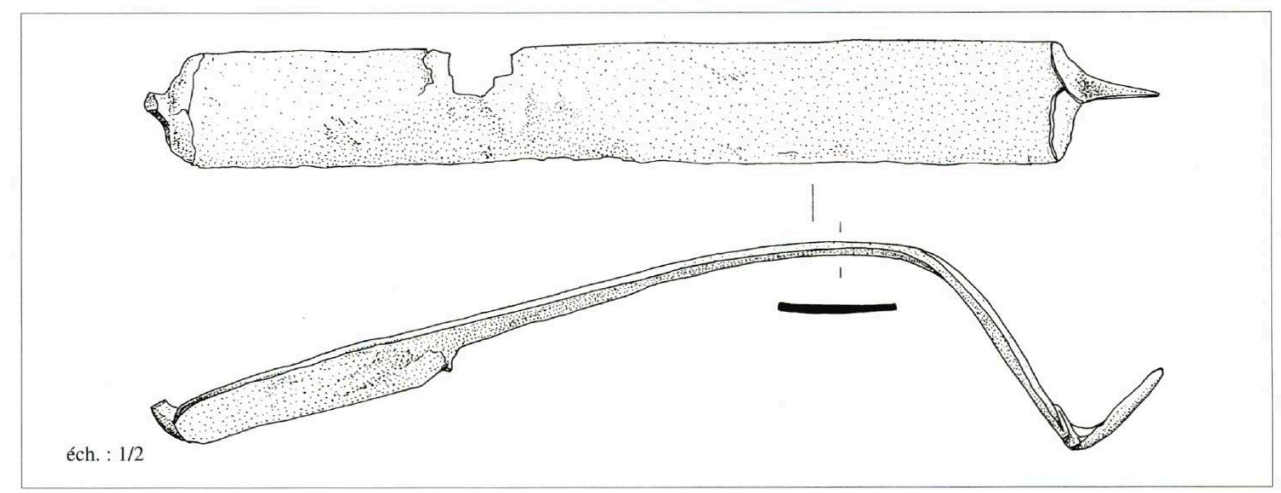

83-Agrafe de charpenterie 


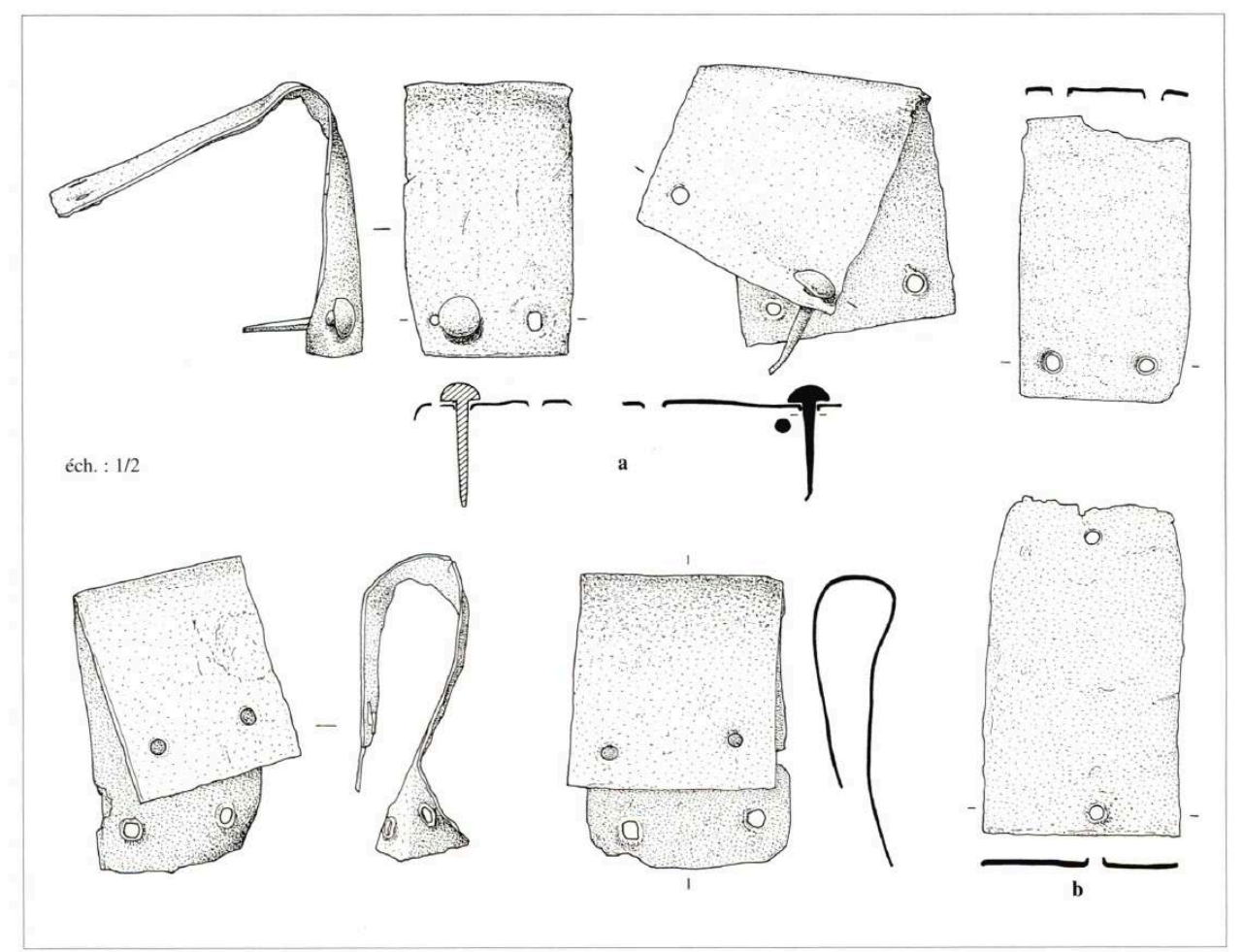

84-Renforcements d'angles en tôle de fer

\section{Tôle rectangulaire à 2 trous}

Une tôle en fer quasi rectangulaire $(7 \times 12 \mathrm{~cm})$ est percée par deux trous sur les petits côtés dont l'un est un peu plus mince $(5,5 \mathrm{~cm})$ que l'autre $(7,1 \mathrm{~cm})(\mathbf{f i g} . \mathbf{8 4} \mathbf{b})$.

\section{Tiges profilées avec anneau}

Deux tiges en fer (long, total 16,1/25,5 cm) portent à une extrémité un disque percé par un clou (dia. 4,2 cm), et à l'autre extrémité un grand anneau ouvert (dia. environ $8 \mathrm{~cm}$ ); les tiges mêmes sont profilées (fig. 85 a). La fonction de ces deux pièces uniques est inconnue. Leur décor témoigne d'un emplacement de choix, peut-être aux essieux du char sensiblement du même diamètre que l'anneau. Toutefois, la présence de deux tiges ne correspond pas aux quatre roues du char.

\section{Petites tôles en fer rivetées}

Deux tôles à languette $(3,3 / 3,7 \mathrm{~cm}$ à la base, $4,2 / 5,8 \mathrm{~cm}$ en hauteur) et à bord courbé vers l'intérieur sont fixées ensemble par deux rivets (long. 2,0 cm) (fig. 85 b). Il existe deux de ces objets dont la fonction n'est pas évidente; ils ne semblent faire partie ni de l'armement (pilum précoce), ni du char.

\section{Bandes en fer de section courbe}

Six bandes en fer de section courbe font partie de l'ensemble, deux sont longues (long. $35,3 / 25,6 \mathrm{~cm}$ ), deux moyennes (long. 18,1/17,5 cm) et deux petites (long. 16,6/13,5 cm); 
5,1-5,4 cm de largeur, sauf la plus courte, qui a 4,5 cm de largeur. Elles ont des trous de clous et un bout pointu. De par leurs tailles et leurs longueurs différentes, ce ne sont pas des garnitures de stabilisation d'essieux (fig. 86).

\section{Longue bande en fer}

Une bande en tôle de fer très longue $(50,0 \times 1,1 / 1,2 \mathrm{~cm})$ a été pliée et repliée plusieurs fois. Sa longueur correspond à la longueur du bassin rectangulaire ; peut-être s'agit-il d'un renforcement du bord de ce dernier (fig. 87).

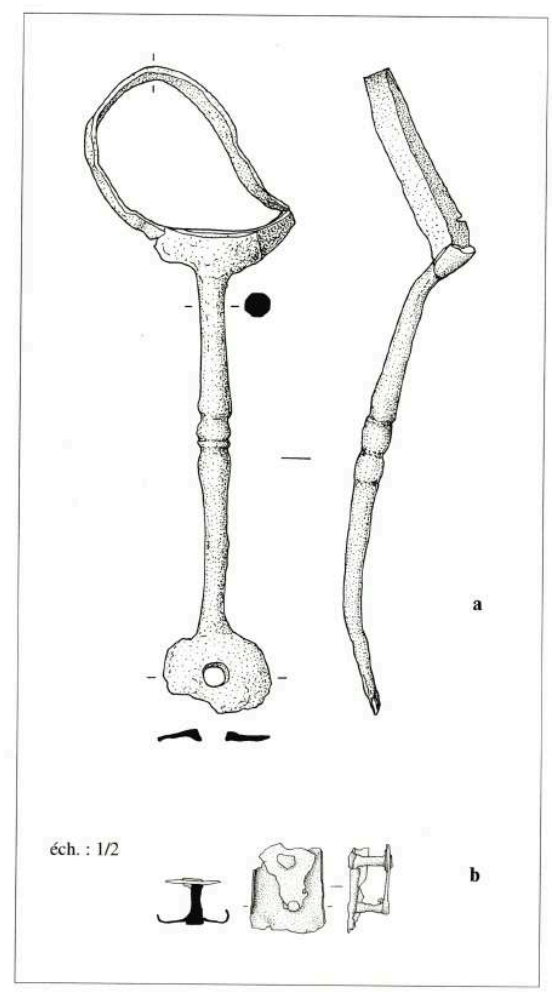

85 a -Tige avec anneau ; $b$ : tôle rivetée

\section{Anneau en fer avec ardillon}

Un anneau en fer (dia. 3,2 cm) de section ronde porte un ardillon pointu également en fer (fig. 88 a). L'objet ne correspond ni aux fibules en forme d'oméga ni aux boucles.

\section{Tiges décoratives}

Deux tiges en fer de section rectangulaire (long. 5,3/5,5 cm) ont des têtes plates et rectangulaires $(2,8 \times 1,7 \mathrm{~cm})$, décorées sur le bord (fig. 88 b). Leur tige est percée d'un petit trou allongé, par lequel il est possible de les fixer de manière réversible. Sur une des tiges, des gouttes de bronze fondu indiquent un contact avec d'autres éléments métalliques, peut-être du char. Aucune fonction ne peut leur être attribuée. 


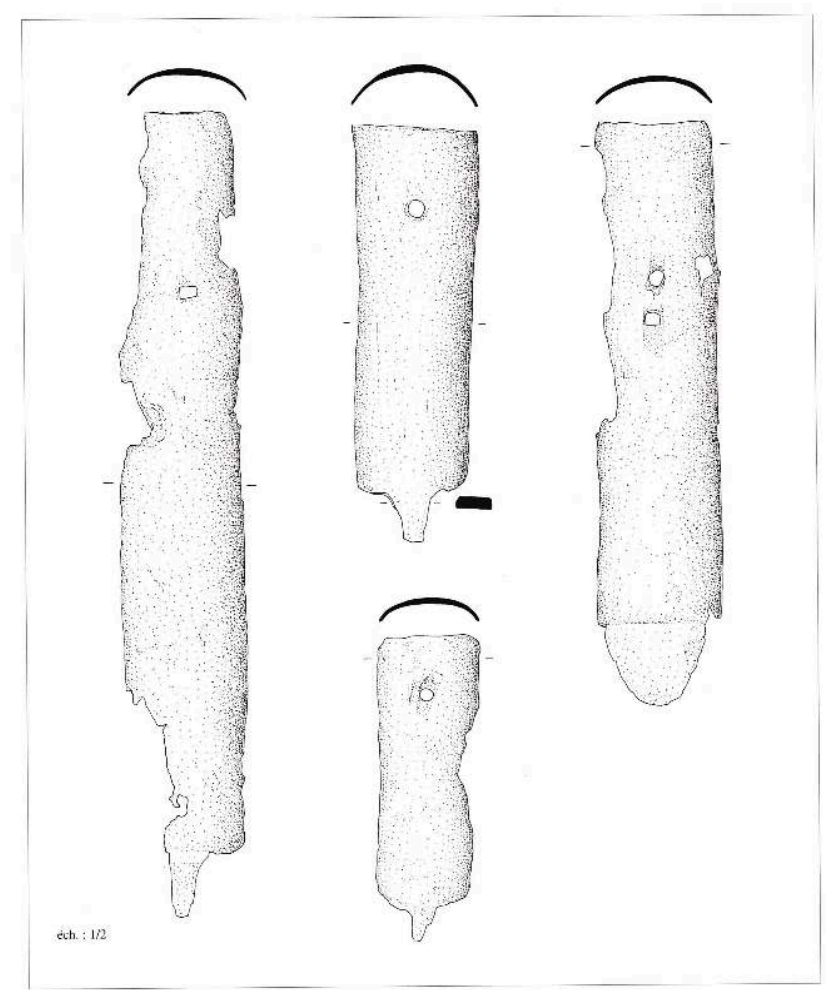

86-Bandes courbées (fer)

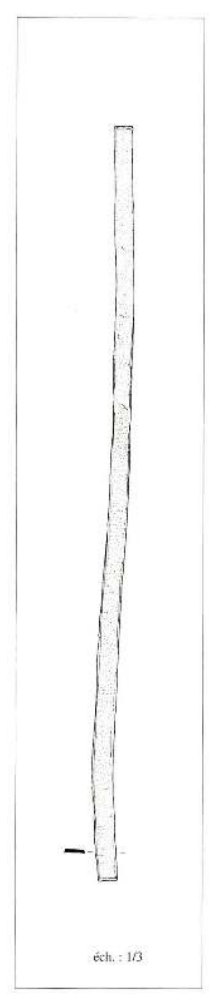

87 - Bande en fer 


\section{Petit ciseau ( ?)}

Un petit ciseau ( ?) en fer est long de 5,4 cm seulement ; sa tête rectangulaire (1,8 x 1,6 $\mathrm{cm})$ a été beaucoup utilisée.

\section{Clous}

- clou décoratif (long. 2,6 cm) à tête allongée (larg. 3,6 cm) avec des rainures pour l'émail (fig. $88 \mathrm{c})$.

- clou décoratif (long. 5,4 cm) à tête allongée (larg. 2,4 cm).

- petits clous à tête plate (fig. $88 \mathrm{~d}$ ).

- petit clous à tête hémisphérique (fig. 88 e).

- clous fins avec tige de section ronde, long. env. $7 \mathrm{~cm}$, partiellement courbés (fig. 88 f).

- clous à pointe plate et à tête ronde, normalement étroits (fig. 88 g).

- clous pointus de section rectangulaire et à tête ronde, souvent pliés deux fois (fig. $\mathbf{8 8} \mathbf{~ h}$ ).

- clous pointus, souvent courbés.

- clou à tête ronde (long. 7,6 cm) de section massive.

- rivet à tête ronde et contre-tête rectangulaire (long. 7,5 cm).

- fragments divers de clous.

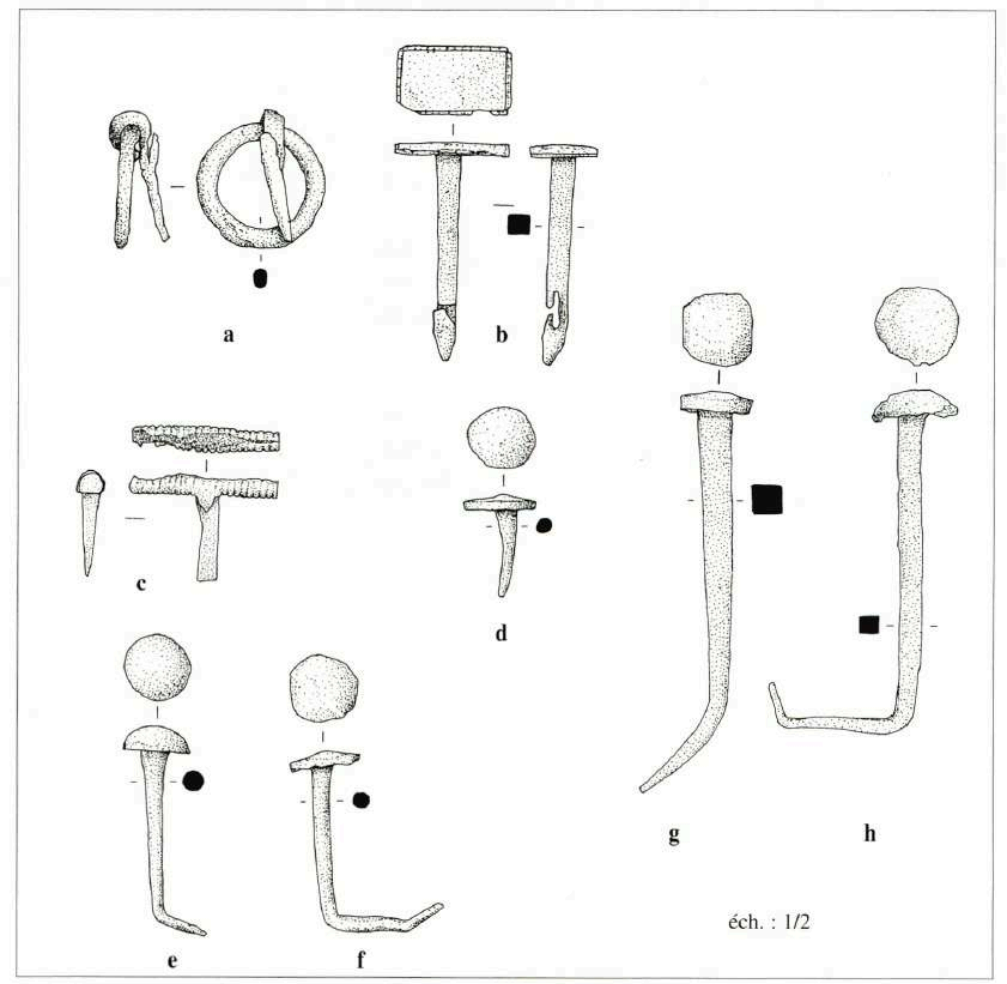

88- Anneau, tiges et clous

\section{LE MOBILIER CÉRAMIQUE. V. Guichard, F. Olmer}

A côté de nombreux objets métalliques, le mobilier de la collection comprend quelques dizaines de tessons de céramique. Infiniment moins spectaculaire, une partie d'entre 
eux peut néanmoins être attribuée de façon plausible à la sépulture et contribuer notamment à préciser la datation de celle-ci.

\section{CATALOGUE}

\section{CÉRAMIQUE CAMPANIENNE}

On dénombre vingt tessons qui appartiennent à quatre récipients au moins. Tous sont assez fortement brûlés, ce dont témoigne une argile de couleur toujours plutôt grise, mais rarement uniforme. Les cassures modernes semblent rares et les remontages sont assez nombreux, ce qui conforte l'impression que l'on a affaire à un nombre réduit de pièces de vaisselle.

1. Assiette en céramique campanienne A à marli ondulé, forme Lamb. 6 (fig. 89 a). Représentée par deux petits tessons jointifs du rebord et une partie du pied. Ce dernier possède un anneau de pose de profil anguleux. Les surfaces sont mal lissées, le vernis mat. Le fond présente un décor de profonds sillons concentriques. Une légère impression peut aussi laisser suggérer la présence d'un timbre. Le diamètre restitué du récipient est d'environ $20 \mathrm{~cm}$.

2. Assiette en céramique campanienne B à lèvre redressée avec un raccord incurvé, forme Lamb. 5 (fig. 89 b). Représentée par un gros tesson de rebord pourvu de trois trous de réparation. Les surfaces sont lisses, le vernis noir mat. Le diamètre restitué du récipient est d'environ $23 \mathrm{~cm}$.

3. Plat en céramique campanienne B de forme Lamb. 5 (fig. 89 c). Représenté par deux fragments non jointifs du rebord. Les surfaces sont lisses, le vernis noir mat. Le diamètre du récipient, non restituable précisément, est d'au moins $30 \mathrm{~cm}$.

4. Plat en céramique campanienne B de forme Lamb. 5 (fig. 89 d). Représenté par six tessons jointifs attenant au rebord. Les surfaces sont lisses, le vernis noir mat. Le diamètre du récipient, non restituable précisément, est d'au moins $30 \mathrm{~cm}$. 4bis. Fragment de fond de plat en céramique campanienne B (fig. 89 e). Il appartient sans doute à l'un des deux plats ci-dessus. Il est doté d'un anneau de pose en bourrelet pourvu d'une rainure sur le dessous et d'une gorge sur sa face externe. La face interne porte un décor guilloché.

Les sept tessons non attribuables à l'un de ces récipients appartiennent tous à des parois d'assiettes et de plats en céramique campanienne B.

\section{CÉRAMIQUE COMMUNE}

La céramique commune est moins abondante. Elle correspond à des récipients de facture et d'état de conservation très variables.

5. Vase haut très altéré par sur-cuisson (fig. 89 f). Les deux tessons présentent un décor de cônes protubérants en fort relief réalisés par estampages répétés, sans doute avec apport de matière dans la matrice. La forme n'est pas restituable.

6. Petit vase ovoïde à fond plat (fig. 89 g). Les cinq tessons jointifs permettent de reconstituer la panse sur toute la hauteur. Une amorce de cannelure devait souligner le départ du col. La pâte, non calcaire et grise, est sans doute partiellement altérée par sur-cuisson. La surface externe est simplement égalisée. 
262 7. Rebord de pot à cuire (fig. 89 h). La pâte, fortement micacée, est assez fine et ne présente pas de trace de surcuisson. Le récipient a été modelé sans tour. La finition extérieure de la panse est seulement égalisée. Le col rentrant et orné de trois cannelures est surmonté d'une lèvre évasée simple.

263 8. Bouton de couvercle (fig. 89 i). La pâte, mi-fine et non calcaire, est gris clair à âme gris sombre. Les surfaces enfumées ne portent pas de traces de sur-cuisson. Les traces de montage au tour sont nettes.

264 9. Petit godet (fig. 89 j). Ce récipient, intact, est modelé dans une argile non calcaire assez fine et fortement micacée. Les surfaces, partiellement égalisées, laissent visibles des coups d'outils destinés à dégager le fond. La surface externe, gris sombre, ne porte pas de trace de surcuisson. La surface interne est uniformément oxydée.

265 10. Cruche (non figurée). Ce récipient est seulement représenté par deux petits fragments jointifs de panse. La pâte, beige clair, est calcaire. Elle ne présente pas de traces évidentes de sur-cuisson.

\section{Amphore}

266 11. Deux petits tessons, jointifs malgré des cassures anciennes, appartiennent à la panse d'une amphore vinaire italique, plus précisément originaire d'Albinia à cause de sa pâte rose très serrée pourvue de très nombreuses petites vacuoles tapissées de calcite et d'inclusions émoussées d'oxyde de fer (Peacock 1977 ; Thierrin-Michael 1992). Les deux tessons portent des traces bien nettes de sur-cuisson.

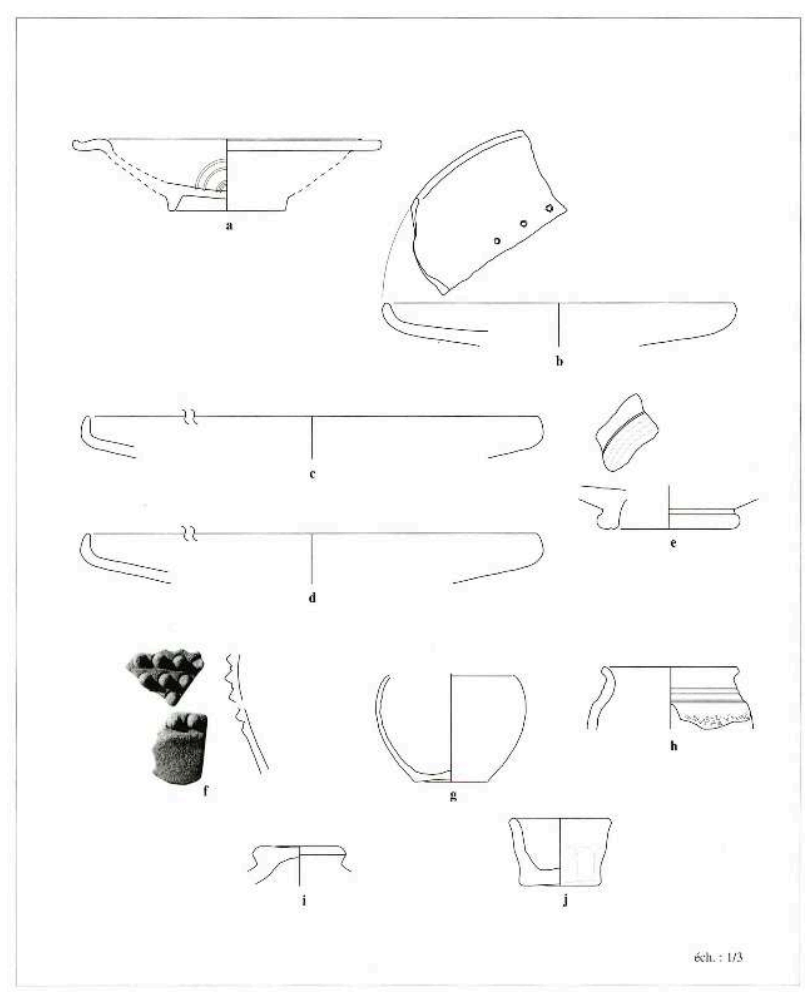

89- Céramique campanienne et commune 
Tuile

267 12. Il s'agit de deux fragments jointifs d'une imbrex (dimension maximum de la partie conservée : $10 \mathrm{~cm}$ ). La pâte fine rose vif évoque celle de certaines amphores vinaires italique, ce qui invite à ne pas exclure l'hypothèse d'une importation.

\section{ANALYSE}

268 Les plats et assiette en céramique campanienne "du cercle de la B" sont un mobilier régulièrement signalé sur les sites gaulois de La Tène finale. La forme Lamb. 5 est la plus classique de ce répertoire. Elle est diffusée à partir du milieu du IIe s. av. J.-C., pour devenir bien plus fréquente au siècle suivant. Nos exemplaires présentent tous les détails caractéristiques des productions tardives, particulièrement fréquentes en Gaule : vernis mat, lèvre allongée non rentrante, pied en fort bourrelet. La céramique campanienne A fournit de la vaisselle dont les importations commencent plus précocement et qui sont concurrencées au Ier s. av. J.-C. par la campanienne B. Notre exemplaire se distingue toutefois par plusieurs caractères qui en font indiscutablement un exemplaire tardif: finition peu soignée, décor de sillons et forme d'apparition récente, attestée par exemple à partir du milieu du IIe s. dans le Midi, mais surtout fréquente dans cette région au siècle suivant (Dicocer $2: 443-447$ ).

269 Au total, les quatre pièces de vaisselle à vernis noir peuvent fort bien être issues du même ensemble funéraire. Bien que le phénomène ne soit pas perçu en Gaule méridionale, où la céramique campanienne A reste largement majoritaire tout au long du Ier s. av. J.-C. (Dicocer $2: 557$ ), on assiste en Gaule interne à un basculement au profit de la campanienne B aux alentours de 100 av. J.-C. (par exemple : Colin 1998 : 73-74). Au vu des ensembles de mobilier disponibles en Gaule du Centre-Est, la coexistence de céramique campanienne $\mathrm{A}$ tardive et de campanienne $\mathrm{B}$ invite ainsi à réduire la fourchette de datation aux phases chronologiques La Tène D1b et La Tène D2a, soit l'extrême fin du IIe s. et les trois ou quatre premières décennies du Ier s. av. J.-C. Parmi les ensembles de référence bien caractérisés, on peut mentionner ceux qui se rattachent à la fin de l'horizon 3 et surtout à l'horizon 4 de Roanne (Morel 1997 : 126-127). En Basse-Auvergne, ce faciès est postérieur à la désaffection des sites de plaine de La Grande Borne et du Pâturai, où la céramique campanienne B est quasiment absente. Il trouve en revanche des correspondances sur les sites d'oppida de Gergovie et surtout de Corent (Guichard et al. 1993: 31-32). De façon plus générale, il est abondamment représenté sur les sites d'oppida du Centre-Est, notamment au MontBeuvray (Barrai, Colin, Luginbühl 1998), mais aussi en territoire allobroge, tant à Vienne (Chapotat 1970 : 119-124), qu'à Larina (Morel 1990).

Le décor estampé original du vase est une variante inhabituelle du décor ocellé fréquent au Ier s. av. J.-C. en Gaule du Centre-Est (Arcelin 1981 : 61-62). Fr. Artaud s'est intéressé en particulier à ce fragment de vase qui figure sous la forme d'un croquis dans son Manuscrit 101/3, ainsi que sur la planche I prévue pour accompagner la publication de la tombe (fig. 3, objet c). Les comparaisons précises sont rares dans le contexte régional.

271 Outre un fragment du site de Saint-Paulien en territoire vellave (Simmonet 1983:101, fig. $5, \mathrm{n}^{\circ}$ 8), on peut signaler un tesson à Soyons (Ardèche) (exposé au Musée Municipal), deux autres près d'Aoste et à Voiron (Musée d'Aoste et informations J.-P 
Jospin), ainsi qu'à "Pian" "sur les terres de M. Nugues, président à la cour royale de Lyon... près de Bourgoin département de l'Isère". Ainsi, ce type encore mal connu se rencontre sur un axe allant des Vellaves aux Allobroges, territoire sur lequel il est le mieux représenté. Constatant de plus qu'il a été fortement dégradé par le feu, son appartenance à la sépulture est plausible.

Le petit vase ovoïde $n^{\circ} \mathrm{g}$ présente une forme trop ubiquiste pour que l'on puisse préciser sa datation, qui ne saurait du moins être antérieure à La Tène finale. On peut notamment penser à une imitation de gobelet à parois fines tardo-républicain ou augustéen, mais la perte de la lèvre et l'absence de décor interdisent de trancher. Une datation plus tardive est tout aussi envisageable.

Le pot à cuire $n^{\circ} h$ possède une forme bien connue dans la région lyonnaise et viennoise au Haut-Empire, qui se caractérise par son col rentrant cannelé. Il semble apparaître après le tournant du IIe et du Ier s., si du moins son absence est significative dans le comblement du fossé lyonnais du clos du Verbe incarné (Sandoz 1989: 67-69). Il est ensuite bien représenté à Vienne au Ier s. av. J.-C. (Chapotat 1970, pl. 46-47) et déjà fréquent à Lyon dans un contexte immédiatement postérieur au milieu du même siècle, avec des exemples encore pour la plupart façonnés sans tour (Mandy, Monin, Krausz 1990 : fig. 9, n 38-42 et fig. 10, n 59).

Le couvercle $\mathrm{n}^{\circ} \mathrm{i}$ présente une facture qui semble exclure une datation au Ier s. av. J.-C. On préfère proposer une datation à l'époque impériale, sans plus de précision. Son appartenance à la sépulture semble donc à exclure, ce d'autant plus que le fragment conservé ne présente pas de traces de sur-cuisson. De plus, l'une des planches figurant en collection privée illustre ce couvercle associé par erreur au vase campaniforme. Des inhumations sous tuiles auraient été découvertes dans le tumulus qui a donc été réutilisé à l'époque gallo-romaine tardive.

Le petit godet $\mathrm{n}^{\circ} \mathrm{j}$ a une forme parfaitement ubiquiste. La pâte et le mode de finition semblent toutefois exclure son appartenance à une phase ancienne de la protohistoire. Par ailleurs, l'oxydation de la surface interne invite à y voir une lampe à suif, sans que cela soit une certitude en l'absence de comparaison précise. Des vases analogues figurent dans le mobilier du dépôt de la Chuire (information F. Perrin, inédit).

très variée de production méditerranéennes échelonnées durant le millénaire qui encadre le changement d'ère.

deux fragments d'amphores appartiennent très vraisemblablement à une amphore Dressel 1, un type abondamment importé en Gaule aux IIe et Ier s. av. J.-C. On note plus précisément que les importations d'Albinia, sur la côte d'Etrurie, sont particulièrement fréquentes en Gaule. L'appartenance de ces tessons à la sépulture est corroborée par les traces de sur-cuisson qu'ils portent.

ne pourrait enfin envisager raisonnablement l'appartenance du fragment de tuile à la sépulture que s'il s'avérait qu'il s'agit effectivement d'une importation méridionale.

On peut finalement classer la céramique étudiée en trois catégories :

- attribution à la sépulture plausible, en raison de critères typologiques et taphonomiques (traces de surcuisson) : quatre plats et assiettes en céramique campanienne (a-e), un vase haut à décor estampé (f) et une amphore Dressel 1 ;

- attribution à la sépulture seulement envisageable, en l'absence de critères typologiques rédhibitoires : vase ovoïde $(\mathrm{g})$, godet $(\mathrm{j})$, vase à pâte claire ;

- attribution à la sépulture à exclure : pot à cuire (h), couvercle (i), imbrex. 
Du point de vue chronologique, ce mobilier apporte des données complémentaires de celles des objets métalliques. Il incite notamment à ne pas faire remonter avant l'extrême fin du IIe s. la mise en place du dépôt funéraire.

En ce qui concerne la cérémonie funèbre, on remarque que les récipients ont tous été exposés au feu du bûcher. La présence d'une amphore vinaire dans cet ensemble n'est pas surprenante, tant c'est une constante des sépultures aristocratiques gauloises de la fin de l'âge du Fer. On s'étonnerait plutôt de ne retrouver que deux discrets tessons d'amphores alors que la vaisselle à boire en bronze est ici exceptionnellement abondante, mais il faut certainement compter sur le mode de prélèvement sélectif des fouilleurs du XIXe s. De ce point de vue, on doit imaginer que le site de la sépulture demeure encore aujourd'hui jonché de tessons d'amphores, ce qui pourrait aider à sa localisation.

La présence de récipients de présentation en céramique campanienne n'est pas surprenante dans un tel contexte aristocratique. Ce genre de dépôt est très fréquent dans les sépultures privilégiées du Midi (Bats 1990 : 273-275), où les assiettes et plats sont également privilégiés vis-à-vis des autres formes. Il l'est bien plus rarement en Gaule chevelue. Sans viser à l'exhaustivité, signalons pour La Tène D, les ensembles suivants, tous inédits : deux incinérations de la nécropole de Feurs" Rue Saint-Antoine" (fouille Valette, 1984), une inhumation d'Aulnat-Gandaillat à Clermont-Ferrand (fouille Vermeulen, 2001) et une incinération de Brioude (fouille Hettiger).

Aussi modestes soient-ils, les restes du vase à décor estampé témoignent de liens évidents avec la sphère culturelle allobroge, ce qui ne saurait étonner.

La présence d'une lampe au sein du mobilier funéraire - ce qui ne reste qu'une hypothèse - trouve des comparaisons nombreuses dans le midi de la Gaule à la même époque, avec des lampes à huile en terre cuite (Bats 1990: 277-278). Le phénomène affecte également les tombes aristocratiques d'autres régions (cf. notamment Goeblingen-Nospelt au Luxembourg pour une période un peu plus récente). Il peut parfois se traduire par la présence d'objets plus prestigieux: candélabre (Beaucaire, Boé), ou lanterne (Antran, Neuvy-Pailloux).

\section{NOTES}

14. Bibracte (à corde interne) : Rieckhoff, Fleischer $2001: 98$, fig. 11,23. -Col de Saverne : Fichtl, Adam 2000 : 49, fig. 968-2. -Mont Terri (non daté) : Kaenel, Müller, Furger-Gunti 1984 : 103, pl. 1, 3.

15. Bataille 2001. Voir liste et carte de répartition : Flecht $1998: 32$, fig. 16.

16. Nîmes : Py $1981: 166$, fig. 75 ; Tendille $1988:$ 70, pl. 10, 72. -Saint-Marcel : Fauduet $1992: 243$, fig. 4, 8. -Mâlain : Mediolanum $1988: 307$ n. 1008. -Nanteuil-sur-Aisne : Lambot 1989 : 40, fig. 7 ; Lejars $1996: 93$, fig. 8, 6 .

17. Lambot $1998: 77$, fig. 76 : Acy-Romance tombe I, $103 \mathrm{NM}$ (patelle à manche type Aylesford).

18. Metzler-Zens et al. $1999: 312-313$. Cruche anthropomorphe en bronze de la tombe 47 (30-15 av. J.-C.). 
19. Nuber $1972: 127$ sq. (pas plus tard que 80 ap. J.-C).

20. Lindenthal, Rupp 2000 : 173-175, fig. 143 (dernier tiers du IIe s. ap. J.-C.).

21. Boube in: Feugère, Rolley (ed.) 1991: 19-20: type Beaucaire. -Fitzpatrick 1987: type Fällanden.

22. Voir par exemple des graffitis (chiffres) sur d'autres vaisselles romaines : Künzl in : Künzl $1993: 400$ avec fig.

23. Wegewitz 1982 : 43 sq. -Fitzpatrick $1987: 101$ : un fragment d'os brûlé indique peut-être une incinération.

24. Références pour des situles type Eggers 18 : Bolla in : Feugère, Rolley (éd.) 1991 : 13 sqq. Suppléments : Gars-Thurnau (A) : Urban in : Neugebauer (éd.) 1992 : 121, fig. 47, 3. -Kelheim (Lkr. Kelheim, D) : Pauli 1993, pl. 115 B 2. -Schwangau (Lkr. Ostallgäu. D) : Maier 1985 : 242, fig. $9,1$.

25. Salzani 1983/84 : 370, pl. 7, 2. -Keiling $1984: 85$, pl. 17,99. -Pour des attaches plus grandes en bronze voir van Endert $1981: 81$.

26. Wielowiejski 1985 : 157. -Bolla in: Feugère, Rolley (éd.) 1991 : 12-13. L'identification du fragment d'une attache de Jacobi 1974, 135 f. Nr. 629 et 631 n'est pas assez sûre.

27. Wielowiejski 1985 : 157 ; Bolla in : Feugère. Rolley (éd.) 1991 : 12 fig. 5. -Nothdurfter 1979: 67 $\mathrm{n}^{\circ} .668$.

28. Raddatz 1969 : 268 : Récipient d'un trésor monétaire de terminus post 104 av. J.-C.

29. Vidal In : Feugère, Rolley (éd.) 1991, fig. 2,5, 8, 20,21, 19, 314-315.

30. Pour la typologie voir Ulbert 1960 ; Feugère in : Feugère, Rolley (éd.) $1991: 53$ sqq.

31. Voir les exemplaires de Manching (Ulbert $1960: 70$ ) et de Casalandri tombe 46 : Salzani 1998 : 93, pl. 27 A, 2.

32. Par exemple la tombe 142 de Wederath (Haffner 1971, pl. 29). - La tombe de Casatenova "Cascina Cacciabuori" (Prov. Como, I) avec gobelet et passoire : Tizzoni 1981, pl. 20.

33. Voir la liste des gobelets de type Idria, variantes Manching et Ornavasso, et des anses séparées: Feugère 1991. - Suppléments: Maria Lankowitz "Primaresburg" (VB Voitsberg, Steiermark, A) : Renseignement D. Kramer (Graz). -Dunaújváros/Intercisa (Kom. Fejér, H) : Alföldi et al. 1957 : 206 pl. 39,10. -Titelberg (L) : Metzler 1995, fig. 170,9. - Ciringhelli (Prov. Verona, I) : Salzani 1983/84, pl. 7, 2. -Forette (Prov. Verona, I) : Salzani 1983/84, pl. 14, 2. - Povegliano "Marinare" (Prov. Verona, I) : Salzani 1983/84, pl. 15, 2.

34. Déjà mentionné par Boube in : Feugère, Rolley (éd.) 1991 : 41 : Hières-sur-Amby, tombe à char de Larina (Isère), $1818: 1$ cruche (rens. J.-P. Guillaumet).

35. Boube in : Feugère, Rolley (éd.) 1991 : 40. -Rieckhoff 1998 : 507 : Lt D1b-Lt D2a.

36. Suppléments à la liste de Feugère in: Feugère, Rolley (éd.) $1991: 41$ : Titelberg (L) : Metzler 1995 : 328, fig. 170, 2. -Raitenbucher Forst (Lkr. Weißenburg-Gunzenhausen, D) : Römer $2000: 312$. 37. Thill 1967, pl. 1, 2. ; Baratte et al. 1984 : 91, pl. $44 \mathrm{n}^{\circ} .134$.

38. Yverdon, chantier des Philosophes $n^{\circ} 13$; Catalogue $n^{\circ}$ 1038. K6169-17. -Aimable renseignement de C. Brunetti.

39. Schulten 1929 : 222, pl. 43,11 (merci à M. Luik, Klagenfurt, pour ce renseignement) et Luik $2002: 226 \mathrm{n}^{\circ} \mathrm{R} 108$.

40. Feugère, De Marinis in : Feugère, Rolley (éd.) 1991 : 108-112 (liste), suppléments : Wehrberger, Wieland 1999 : 253-254 note 64 ; pour l'Espagne : Mansel $2000: 215-218$.

41. Feugère, De Marinis in : Feugère, Rolley (éd.) $1991: 112$. -Voir une patelle à manche entier au Musée de Nîmes: Tendille 1988: 37 ( $\mathrm{n}^{\circ}$ 124, Provenance inconnue; support en forme de coquillage).

42. Goeblingen-Nospelt : Thill 1967 : 69, pl. 1,4.-Gautzsch : Eggers 1965. -J. Metzler a attribué des pieds des patelles à manche à une réparation locale ("gallische Erganzung") -Metzler 1995 : 329, note 652 .

43. Bolla in : M. Feugère, Cl. Rolley (éd.), 1991 : 148 avec contribution de Cl. Rolley p. 152-153. Metzler-Zens, Metzler 1998 : 419, fig. 1-2. 
44. Künzl in : Horn, Rüger 1979 : 295-300. -Voir Eggers type 95 : Eggers $1951: 168$ sp.

45. Voir les vaisselles avec un bord simple et haut de Pompéi : Tassinari $1996: 115$, fig. 2 sq.

46. Voir Eggers type 90 : Eggers 1951. pl. 9. 90. -S. Künzl in : Künzl (éd.), 1993 : 197-214.

47. Eggers 1951: 167 : Type 73 : Korchow tombe 96, Harsefeld, tombe 126. -Type 74 : Harsefeld; Övra Ålebäck, tombe 10 (Öland, S) -pour la dernière tombe voir Rasch 1994. Le vase est sans doute en fer.

48. Eggers $1951: 41$ : il parle de la possibilité de "l'erreur de petit chiffre".

49. Voir des autres exemples à Clémency et à Goeblingen Nospelt tombe A et B (Metzler et al. 1991 : 99).

50. Manching : van Endert 1991 : 46-52. 91, pl. 9, 237. 27. 448 ; peut-être : Basel/Münsterhügel : Furger-Gunti, Berger 1980 : 75, pl. 11, 27 (tôle trop mince ?).

51. Aimable renseignement de N. Ginoux, Paris.

52. Van Endert 1991 : 46-52 -Voir aussi le bec verseur zoomorphe de Hellingen, tombe B (L) : Krier, Reiner 1993 : 21. Il indique une continuité de la production à l'époque romaine.

53. L'hypothèse campanienne est déjà évoquée par C. Rolley 1983 : 140, conjointement à la possibilité d'une fabrication sur la côte adriatique elle-même, qui nous paraît moins probable, compte tenu du style sensiblement différent des figurines en bronze connues dans ce secteur.

54. Gauer 1991 : pl. 22 (Le 98, 99. 101) ; pl. 27-28 (Le 414 et 415). Sur la caractérisation des produits en bronze campaniens au début du Ve s.: A.-M. Adam. Mélanges de l'Ecole Française de Rome (Antiquité), 92, 1980 : 641-679.

55. Par exemple les cavaliers d'un chaudron du British Museum ( $\left.{ }^{\circ} 558\right)$ : Rolley 1983 : 140, fig. 132.

56. Castel S. Mariano: U. Höckmann, Die Bronzen aus dem Fürstengrab von Castel San Mariano. Munich, 1982: 99, n 57; Monteleone di Spoleto: Scarpellini 1979: 373, fig. 13; Orvieto, Crocefisso del Tufo, t. 17 : Scarpellini 1979 : 373, fig. 12 ; S. Giovanni in Compito : Scarpellini 1979 : 367-372.

57. M. Novotna, Die Bronzegefdsse in der Slowakei. PBFII, 11 ; Munich. 1991, $\mathrm{n}^{\circ} 65$ : 71-72, pl. 13.

58. A Rudiae, Rutigliano. Cavallino. Ugento : Rolley 1991 : 190-196. Sur les récipients grecs, la première synthèse est celle de M. J. Milne 1944; sur leur usage: R. Ginouvès, Balaneutikè. Recherches sur le bain dans l'Antiquité grecque. Paris, 1962 : 61-75.

59. A Olympie : Gauer 1991 : P 5, pl. 56, 1.

60. Gauer 1991 : pl. 57, 3 et 4 (Olympie. P 32 et P 38); pl. 61, 3 (P 34).

61. Bassin complet de Sala Consilina : Gauer 1981 : 117, fig. 53 ; anse isolée à Olympie (Br 5176) : Gauer $1991: 27$, pl. 62, 2.

62. Gauer 1981: 120, note 28 ; A. De Ridder, Catalogue des Bronzes trouvés sur l'Acropole d'Athènes. Paris, 1896, n²06, p. 67 sq., fig. 40-41 (le décor est complété par deux gorgoneia sous les éléments d'attache incurvés).

63. British Museum, $n^{\circ} 73.8-20.217$ : Haynes 1985. $n^{\circ} 39: 259$, fig. p. 146.

64. Br 5110 : Gauer 1991 : P 26, pl. 62, 1.

65. Hostteter 1986 : Spina 40, p. 73-75, pl. 49 (discophore de la tombe 44 ; datation proposée : 440-430) ; Spina 46, p. 80-81, pl. 56 (kyniskos de la tombe 65A; datation: 400-375) ; figures d'Hermès des deux candélabres jumeaux, Spina 16 et $17:$ p. 41-43, pl. 19-22 (tombe 136A, datation : vers 400); athlète avec strigile de Servirola S. Polo : Mostra 1960, n 809, p. 245, pl. LX (la datation proposée dans ce catalogue -début du IVe s. -est sans doute un peu basse). Sur la petite plastique tardo-classique d'Etrurie padane, en dernier lieu: Sassatelli 1987; Jurgeit 1986: 115-117 ; F. Gilotta, Nota di plastica spinetica. Prospettiva, 77, 1995 : 51-57.

66. Cristofani $1985: 165, \mathrm{n}^{\circ} 57$ (Monte Capra) ; 120-121, n 7. 4, (Villa Cassarini).

67. Cristofani $1985: 104, \mathrm{n}^{\circ} 4.7$, (grande statuette d'offrant, vers 400 av. J.-C.) et $\mathrm{n}^{\circ} 4.8$, p. 105 (jeune homme nu, une épée en bandoulière, 400-370) ; petit bronze votif considéré comme "nordétrusque" au British Museum : Haynes 1985 : 214 et 302, n 155,. 
68. Par exemple le célèbre groupe de Marzabotto "Mars et Vénus" : Maule 1977 : 498, fig. 12.

69. Guerrier de Falterona : Cristofani $1985: \mathrm{n}^{\circ}$ 4.6, p. 102-103 (datation : 420-400)

70. L'un autrefois dans la collection Sarti à Rome : F. Messerschmidt, Römische Mitteilungen, 43, 1928, p. 153, fig. 3 ; l'autre au Römisch-Germanisches Zentralmuseum de Mayence (Inv. $\mathrm{n}^{\circ}$ 0.8919) : Maule $1977: 498$, fig. 13.

71. Landolfi 1987 : 452-454 ; M. Menke 1991 : 392-393 proposent une date plus récente, à la fin du IVe s.

72. Jannot $1995: 83$. Le cliché que nous publions, qui correspond à un cliché ancien, antérieur à la plus récente restauration, montre en tout cas des reliefs plus marqués et un style plus conforme au reste de la série.

73. Rome, Musée de la Villa Giulia, $\mathrm{n}^{\circ} 15693$ : Bordenache Battaglia 1979 : $\mathrm{n}^{\circ}$ VIII, p. 18-19 et pl. XXII. Le type des pieds (K 29) est également daté par F. Jurgeit (1986: 119-150) de la première moitié du IVe s. (dernière période d'activité de l'“atelier A").

74. Bordenache Battaglia 1979 : $\mathrm{n}^{\circ}$ 30, p. 115-116 et pl. CXLI (Londres, British Museum, $\mathrm{n}^{\circ} 84$ 6-14 $35) ; n^{\circ} 22$, p. 95-97 et pl. CXIII (Karlsruhe, $n^{\circ}$ F 1859). Les poignées qui présentent un couple de lutteurs mixtes ("Pélée et Atalante") sont les plus récentes (fin du IVe s.).

75. Cristofani 1985 : 34-35. Cette proximité est bien illustrée par les poignées de cistes ajourées figurant des soldats en armes (Rome, Villa Giulia, n² 25210 : Bordenache Battaglia 1979 : $n^{\circ}$ VII, p. 16-17, pl. XVI-XVII) ou encore deux personnages nus, portant le corps d'un troisième, qui ont été justement rapprochés de l'éphèbe "Fruga" de Monte Capra (Copenhague, Ny Carlsberg Glyptotek, $\mathrm{n}^{\circ} \mathrm{H} 241$ : Bordenache Battaglia 1979 : $\mathrm{n}^{\circ}$ III, p. 8-9 et pl. VI) ; pieds et poignée de cette ciste sont datés par F. Jurgeit (1986:128-129) du premier quart du IVe s.

76. Fouilles Karine Robin, 2001: K. Robin et C. Soyer, Un fragment d'anse de bassin étrusque découvert à Barzan (Charente-Maritime), à paraître dans Aquitania, (je remercie K. Robin et C. Soyer qui m'ont signalé cette découverte et permis de l'examiner).

77. F.-R. Herrmann et O.-H. Frey. Germania 75, 1997, 2, p. 459-550.

78. Type recensé par L. Husty, Ein neuer etruskischer Gefässtyp. Trierer Zeitschrift, 53, 1990 : 15-54, qui regroupe ces récipients dans la classe des kyathoi “de type A, variante Bologne".

79. U. Schaaff, In : Künzl 1993 : Teil I. p. 66-68, carte fig. 3, Medilerrcme Bronzegefässe und Spiegel des 4./3. Jahrhunderts v. Chr. in Gallien. Sur cette carte figurent aussi l'anse de Borsdorf et celle de Sainte-Gemmes-sur-Loire.

80. Publication de cette oenochoé par R. Chevallier: La troisième route de l'étain en Gaule. A propos d'une oenochoè en bronze étrusque trouvée près de Tours. In: Homenaje a A. Garcia y Bellido, t. 2. Madrid, 1976 : 131-157, fig. 1-4.

81. H.-E. Joachim, éd., Waldalgesheim. Das Grab einer ketlischen Fürstin. Cologne, 1995 : 59.

82. B. Shefton, The Waldalgesheim Situla : where was it made ?. In : Festschrift für 0.-H. Frey zum 65. Geburtstag. Marburger Studien zur Vor-und Frtihgeschichte 16 ; Marburg, 1994 : 583-593.

83. C. Rolley, Deux notes de chronologie celtique. II. La situle de Waldalgesheim et la date du Style végétal continu. Revue Archéologique de l'Est et du Centre-Est, 38, 3-4, 1987 : 416-419.

84. S. Künzl, In : Künzl 1993 : Teil I : 69-72, avec carte de répartition du type, fig. 3, p. 71.

85. Th. Weber, Bronzekannen. Francfort/Main, 1983, types II E et F, p. 92-105.

86. Une anse isolée, correspondant à une oenochoè de même forme a été publiée par P. Lebel (Catalogue des Collections archéologiques de Montbéliard. 3: Les bronzes figurés. Ann. Litt. Univ. Besançon vol. 57 ; Paris, 1962, n 52, p. 32 et pl. 41), comme provenant de Mandeure.

87. Nous ne mentionnons que pour mémoire d'autres récipients, qui ont été considérés occasionnellement comme des importations du IVe s., sans fondement à notre avis (et l'authenticité de leur provenance locale est d'ailleurs loin d'être assurée) : une coupe, en fait campanienne, conservée au Musée de Lyon et passant pour avoir été trouvée à Mèves (Nièvre) ; ou encore un gobelet à bec verseur provenant, semble-t-il, de Thorigné-en-Chamy et conservé au musée de Jublains (Mayenne) : Nantes 1999: 80, nº 143. 
88. Quincerot : C. Rolley, Bulletin de Correspondance Hellénique, 1962 :491-493, fig. 14-16 ; Faulx : M. Leroy, In : L'âge du Fer en Lorraine. Exposition Musée de Sarreguemines ; 1987 : 36-37.

89. Cette hypothèse a été retenue par M. Menke (1991) pour tous les bassins à lutteurs, mais elle repose dans son étude sur un postulat de départ non recevable, puisqu'il date tous ces bassins sans distinction (y compris celui de Borsdorf) de la fin du IVe s.

90. Pour le Bassin Parisien : sépulture du Plessis-Gassot (Val-d'Oise), avec deux coupes à vernis noir : N. Ginoux, Archéologia, n 358, juil.-août 1999: 19. Pour la Suisse : Coupe à vernis noir d'Ollon (VD) : G. Kaenel, Recherches sur la période de La Tène en Suisse occidentale. Analyse des sépultures. Cahiers d'Archéologie Romande vol. 50 ; Lausanne, 1990 : 297-298. Sur ces questions de mobilité des individus de part et d'autre des Alpes : D. Vitali et G. Kaenel, Un Helvète chez les Etrusques vers 300 avant J.-C. Archéologie Suisse, 23, 3, 2000 :. 115-122.

91. A Larina : un fragment, fabriqué à Teano (?), daté des environs de 300 ou du premier tiers du IIIe s. (selon J.-P. Morel, In : Perrin 1990 : 114) ; à Vienne : atelier des Petites Estampilles, fin IVedébut IIIe s. (Perrin,. Bellon 1992 : 426, note 55).

92. Les bronzes antiques de Paris. Exposition Musée Carnavalet ; Paris, $1989: 42-43, \mathrm{n}^{\circ} 5$.

93. Nantes 1999 : 74-75, $\mathrm{n}^{\circ} 132$,

94. Un écart chronologique entre les différents éléments du mobilier funéraire a été mis en évidence également pour le tumulus de Strettweg en Autriche : Egg 1996 : 243-245.

95. Sur ce contexte, en dernier lieu : J. Fischer, Germania, 68, 1, 1990 : 115-127.

96. Exception dans la tombe de Waldalgesheim, où l'objet “ancien" conservé n'est pas la situle de bronze grecque, mais l'oenochoè à bec tubulaire, de fabrication celtique.

97. M. Guggisberg, "Erbstücke in fürstlichen Gräbern diesseits und jenseits der Alpen", communication au Colloque Die Hydria von Grächwil, 1851-2001. Zur Funktion und Rezeption mediterraner Importe in Mitteleuropa im 6. und 5. Jh. v. Chr. (Berne, octobre 2001). L'article fondateur sur le sujet est celui de F. Fischer, Keimelia: Bemerkungen zur kulturgeschichtlichen Interpretation des sogenannten Sudimports in der späten Hallstatt-und frühen Latènekultur des westlichen Mitteleuropas. Germania. 51, 1973 : 436-459.

98. Langâ au Danemark, tombes 1 et 2 : P. Stary, Mediterrane Einfuhrgüter während der Früheisenzeit in England und Skandinavien. Römische Mitteilungen, 98, 1991 : 19-21.

99. Flonheim (Kr. Alzey-Worms, D) : Behrens 1923 : 39, fig. 47. -Hofheim am Taunus, tombe 9 (Main-Taunus-Kreis, D) : Ritterling 1912 : 394, fig. 110, 6. -Bad Nauheim, tombe 28 (Wetteraukreis, D) : Quilling $1903: 19$.

100. Voir Graue 1974: 29 sqq. pour les gobelets en argent dans les cimetières à Ornavasso/San Bernardo et Persona, ainsi qu'à Giubiasco.

101. Voir Feugère in : Feugère, Rolley (éd.) 1991 : 78 sqq. liste $3 n^{\circ}$ 52, 57, 68.

102. Voir: Saint-Laurent-des-Arbres (dép. Gard) : Barruol, Sauzade 1969 (casque conique à pointe, de type étrusque avec décor ondé). -Trier-Olewig (Kr. Trier-Saarburg, D) : Schindler 1971 (casque type Mannheim). -Deux autres casques douteux sont mentionnés à Sigoyer (dép. HautesAlpes) : Gallia 43, 1985 : 523; Ulysse, Guillaume 1991: 139, 220 (reconstitution d'un guerrier à casque conique à pointe, de type étrusque avec décor ondé).

103. Voir le casque en bronze de type celtique oriental de Šmarjeta-Vinji vrh (SLO) : Guštin $1984 \mathrm{~b}: 352$, pl. 48.

104. Pour cette nécropole, voir Marcadal 2000.

105. Récemment : Wieland 1996 : 109 sqq. -Haffner 1989. -Schaaff $1985: 623$ fig. 12-13.

106. Chalon-sur-Saône (dép. Saône-et-Loire) : Déchelette 1913 : 175 sq. fig. 25,5, pl. 36,5. -Port (canton de Bern. $\mathrm{CH}$ ) : Vouga 1885, pl. 3,1.

107. Regensburg (Stadt Regensburg, D) : Stroh In : Germania t. 29, 1951 : 141 sqq. fig. 1, 3.

108. Pièce de suspension en bronze (exemple) : Ludwigshafen (Kr. Ludwigshafen, D) : Engels 1970. -Pièce de suspension en fer sur un fourreau en bronze (exemples) : Wederath tombe 296 (Kr. 
Bernkastel-Wittlich, D) : Haffner 1971, pl. 73, 5a. ; Wollendorf (Kr. Neuwied, D) : Oesterwind 1989, pl. $6 \mathrm{~B}, 6$.

109. Par exemple : Giubiasco tombe 96 (Kt. Tessin. CH) : Ulrich 1914 : 573 sq. pl. 76,4-5. -Conthey (Kt. Vaud, CH) : Peyer 1980: 59 sqq. fig. 7, 6. - Regensburg (Lkr. Regensburg, D) : Stroh In : Germania t. 29, $1951: 141$ sqq. fig. 1, 3.

110. Voir: Illkirch-Graffenstaden (dép. Bas-Rhin): Henning 1912: 12 pl. 11,1.- Ormes (dép. Saône-et-Loire) et Champdivers (dép. Saône-et-Loire) : Wieland 1995 : 112 fig. 36,2-3.

111. Müller $1990: 42$ fig. 18 bas (largeur des lames) : 43 fig. 19 (index de longueur et largeur des lames d'épées).

112. Aimable renseignement de U. Schaaff et M. Egg (RGZM).

113. Aimable renseignement de F. Hummel (RGZM), technicien de rayon-X.

114. Titelberg/Lamadeleine tombe 39 avec une pointe effilée en forme de feuille de saule (long. $30,3 \mathrm{~cm}$ ) : Metzler-Zens et al. : 164 fig. 165, 5. - Cottévraed tombe 130 : Blancquaert $1998: 178$ fig 8 (pliée, plus de $30 \mathrm{~cm}$ ).

115. Voir Egg 1996 : 155 sqq. pour la phase Ha D1 dans le domaine Hallstattien oriental.

116. Voir par exemple: Müller-Karpe 1951, fig. 3, 1; 8, 2; 9, 7.

117. Voir par exemple: Bystrzec (Woi. Pomorskie, PL, l'ancien Weißhof, Kr. Marienwerder) : Heym 1959/61 : 162, fig. 5, 8 .

118. Pour la surface de quelques épées de Bern/Tiefenau (canton de Bern. CH) : Müller 1990 : 37.

119. Port : Jahn 1913 -Bludenz : von Merhart 1940 : 87 fig. 1.

120. Voir P. Luczkiewicz, Die verzierten Lanzenspitzen: Spuren germanischer Wanderungen? In : Bewaffnung der Germanen und ihren Nachbarn. Collogue 1999. Lublin 2002, p. 15-40.

121. Behrens $1923: 21$ fig. 25. -Krämer $1985: 137$, pl. 70, 2.

122. Sievers 1995 : 155-156. -Comme parallèle voir Feugère $1996: 179$ fig. 15, 13.

123. Voir Spagnolo Garzoli 1999 : 352 pour des types différents.

124. Tischler 1886 pour le site de La Tène (Vouga 1923, pl. 37, 7); Manching/Steinbichel tombe 35, 36, 40 (Krämer 1985) ; München/Obermenzing tombe 12 (Krämer 1985. pl. 60.6) ; Gournay-surAronde (Challet $1992: 108-111)$.

125. Type Sion : Sion/Saint-Guérin (canton de Genève, $\mathrm{CH}$ ) : Kaenel $1983: 48$ fig. 11, 4. -Mirebeau (dép. Côte-d'Or) : Brunaux et al. 1985 : 93-94 fig. 14.

126. Voir : Marcilly-sur-Eure (dép. Eure) : Duval, Verron 1993 : 139 fig. 5, 2. - Mirebeau (Côted'Or) Brunaux et al. 1985 : 94, fig. 14. - Acy-Romance NMI, 103 (dép. Ardennes) : Lambot $1998: 77$ fig. 76. - Lebach tombe I (Lkr. Saarlouis, D) : fragment d'umbo et manipule (Gerlach 1976: 11 pl. 1 $\mathrm{g} ; 2 \mathrm{k}$ ).

127. Roualet et al. $1982: 31,36$, pl. V b, f: tombe 2/1960 (1 exemplaire). $3 / 1964$ (2 exemplaires d'une tombe de guerrier). - Mentionné d'après Raftery $1988: 52-53$.

128. La Tène: Vouga 1923: 99 pl. 36, 4 (aujourd'hui à Berlin. Museum für Vor-und Frühgeschichte). - Lozna : Teodor 1980 : Babes 1993 : 203 pl. 43, 11.

129. Aimable renseignement de $S$. Sievers, Francfort.

130. Haffner 1989 a: 181. L'épée de Wintrich, pour laquelle Haffner mentionnait un décor semblable (note 11) n'est pas encore publiée.

131. Pour le type Nierstein voir par exemple : Gournay (entre autres $n^{\circ} 1425,1484,1561.1930$, 2706) : Brunaux, Rapin 1988 ; Beckingen tombe 7 (Lt C2) : Miron 1986/87 : 127 pl. 3E ; type Nîmes voir La Villeneuve-au-Châtelot: Piette 1989 : 252 fig. 5a.; Wallertheim tombe 5 (Lt C2) : Kessler 1929/30 : 132 fig. $15,2$.

132. Type Sion : Sion/Saint-Guérin (canton de Genève, $\mathrm{CH}$ ) : Kaenel 1983 : 48 fig. 11,4.-Mirebeau (dép. Côte-d'Or) : Brunaux et al. 1985 : 93-94 fig. 14.

133. Sievers 1995 : 139-140 fig. 30, 1. - Lejars 1996 : 95 fig. 9, 6. - Arcelin 1980 : 100 fig. 12.

134. Type Owlesbury : Collis $1973: 128$ fig. 4, 6. - Soissons : inédit, renseignement D. Roussel, Musée Soissons. 
135. Après les sources historiques: Dobesch 1996 : 14-16. - Pour les données archéologiques : Joachim $1974: 163$; Roymans $1990: 247-249$.

136. Raddatz 1967. -Schönfelder 2000a. -Schönfelder 2000b : 74-76. - Schönfelder (2002).

137. Miniero 1987 : 192. -Voir Dolenz 1998 pour un char du Magdalensberg (Kärnten, A) de l'époque tibérienne tardive. - Pour les chars romains du IIe/IIIe s. voir Visy 1993.

138. Voir par exemple les frettes de Bern/Tiefenau : Müller $1990: 53$.

139. Voir aussi Manching : Jacobi 1974, pl. 56 no. 846.

140. Voir par exemple : Brežice tombe 6 (SLO) : Guštin 1984 : 117 pl. 3, 1-2. - Bouqueval tombe 3 (dép. Val-d'Oise) : Guadagnin 1978 :52, fig. 43. - Plaidt (Kr. Mayen-Koblenz, D) : Joachim 1969, fig. 4, 5-6.

141. Westdeutsche Zeitschrift 19, $1900: 400$ pl. 18, 23 ; Landesmuseum Mainz Inv. Nr. R 5152.

142. Pare 1992 : 129-132. -Egg, France-Lanord $1987: 28$.

143. Voir par exemple : Augsburg/Oberhausen (Stadt Augsburg, D) : Hübener 1973 : 15, 1. 6.14 (p. 43 mentionné comme essieu de char - éventuellement moderne ?). -Haltern (Kr. Recklinghausen, D) : Harnecker 1997, pl. 50 f. N. 580-584. - Vindonissa : Unz, Deschler, Deschler-Erb 1997: 50 pl. 68, 1947-1953 (nommée tige latérale d'un mors). - Etude exhaustive : Visy 1993, 279. - Une tige de pivot de Breisach-Hochstetten (Kr. Breisgau-Hochschwarzwald, D), en vitrine au musée de Breisach comme essieu laténien, a été trouvée dans un puits romain (structure 32/21) et n'a pas de relation avec l'habitat de La Tène finale (aimable renseignement de M. R. Dehn).

144. Renseignements M. F. Hummel, RGZM.

145. Information non publiée, renseignements J.-P. Guillaumet ; $c f$. rapport préliminaire : Quinn 1995 : 240-245 ; Gruel, Vitali (dir.) 1998 : 43 sq.

146. Pour la terminologie des structures des chars, voir Hayen 1983. Le terme Y ou YY présente l'image du timon ou de la longe écartée.

147. Dejbjerg: Petersen 1888, pl. 3, 10 ; 5, 7b, 6e. - Boé : Schonfelder 2000b. - Husby : Raddatz 1967, pl. 5, 1 .

148. Vierrädrige Wagen $1987: 89$ (Ohnenheim, dép. Bas-Rhin), 127 (Hochdorf, Lkr. Ludwigsburg, D), 129 fig. 1 (Como Ca' Morta, Prov. Como, I), 157 (Vix, dép. Côte-d'Or).

149. Molin 1984 : 101 "barres transversales".

150. Haffner, Joachiml984 : 74-75. - Cahen-Delhaye 1993 : 64 sqq.

151. Schönfelder 2002 : 224-244.

152. Schönfelder $2002: 236$, tab. 37, fig. 146.

153. Schönfelder 2002 : 245 sqq. ; Alföldi/Radnöti 1937-40 : 312 pl. 24, 1-6 ; 28, 1-2.- Voiraussi : Piatra Rosie(Jud. Hunedoura. RO) : Daicoviciu 1954 pl. 5, 3.

154. Voir Schönfelder 2000b : 74-76. -Schönfelder 2002 : 352-355 : Dans les tombes : Boé (Lot-etGaronne), San Maria di Zevio/Lazisetta (Prov. Verona, I) und Cugir (Jud. Alba, RO).

155. Vierrädrige Wagen 1987. - Pare 1992.

156. Röring 1983. -Voir comme exemple : Mariën 1994.

157. Timpel 1980. -Wamser 1984.

158. Voir Ronke 1987 : 181 sqq.- Amedick 1993 : 146-147.

159. Amedick 1993 : 146-147. Un “char à fauteuil” est représenté comme insigne aussi dans la notitia dignitatum.

160. Künzl $1988: 85$ sqq. - Versnel $1970: 56$.

161. Canosa di Púglia (Prov. Bari. I) : Frey 1984 : 124 fig. 3. - Rocca San Casciano (Prov. Forli, I) : Galli e l'Italia 1978 : 388 fig. 259, 28.

162. Des fragments en forme d'oméga, qui permettent la fixation des montants, se trouvent dans plusieurs habitats ouverts: Krämer 1964; Wamser 1982; Bayerische Vorgeschichtsblätter, Beiheft 4 (München 1991) p. 119, 126 fig. 77, 3. 
163. Saint-Romain : Bourgogne médiévale $1987: 177$ no. 438 (site médiéval, qui a donné également du matériel laténien : Grappin 1985 : 64 ; renseignement U. Schaaff [RGZM]). - Léry : Normandie $1990: 51$.

164. Voir Ulbert, 1984 : 71 sqq. -Kotera-Feyer 1993. - L'anneau de "Ville-en-Tardenois" (dép. Marne) Bulletin de la Société Archéologique Champenoise 8, 1914, 42 ; Bretz-Mahler 1959, 487-488 n'est pas un torque ornitomorphe.

165. Kull 1996. -Voir aussi un cheval de la phase LT C/D de Sopron (H) - renseignements E. Jerem. 166. Notici Scavi Ser. 8, vol 14, 1960 : 240-245, voir fig. 31.

167. Joachim 1974 - Roymans 1990 : 247 sqq. (“horseman's graves").

168. Sur l'existence des cavaliers à La Tène A en Champagne, voir Verger 1996.

169. Voir par exemple Kull $1996: 425$ et Oesterwind $1996: 86$.

170. Thill 1967. -Metzler et al $1991: 113$ sqq. fig. 86-88.

171. Caes. Gall. II 24, V 3 ; Tac. ann. II 42. - Metzler 1994 : 99. - Oesterwind 1996 : 97-98. -Krier/ Reinert 1993 - Fingerlin 1999.

172. Joachim 1969 : 99. -Joachim 1973 : 40. - Roymans $1990: 249$.

173. Voir par exemple la tombe 16 de Belgrad-Karaburma (YU) : Todorovic 1972, pl. 6. -Roje pri Moravce (SLO) : Knez 1977 : 119 pl. 8. - Bozic, 1984.

174. Voir Adler 1993 : 148 fig. 53. - Völling 1992 - Bockius 1991 : 499 fig. 2 avec liste p. 512 sqq.

175. Lavendhomme, Guichard 1997, 161 pl. 115,2. -Vercingétorix 1994, 112 fig 117r. -Jacobi 1974, 240 pl. 80, 1583-1587.

176. Jacobi 1974, pl. 64, 1122-1123.

177. Pour La Tène A en Champagne : voir Joffroy, Bretz-Mahler $1959: 20$ fig. 15, en Belgique voir Cahen-Delhaye 1993 : 62 fig. 6. - Pour La Tène $C$ et $D$, voir : Bern/Tiefenau (Kt. Bern, CH) : Müller 1990 : 52 pl. 18, 130 ; 41,1113. -Manching (Lkr. Pfaffenhofen a. d. Ilm, D) : Jacobi 1974 :211 sq. No. 828-829. -Dejbjerg (Ringkøbing amt, DK) : Petersen 1888, pl. 1, 2. ; 4, 1c. - Husby (Kr. Flensburg. D) : Raddatz 1967, pl. 3, 5-8. - Stradonice (Bez. Beroun, CZ) : Pič 1906, pl. 22, 18. 


\section{Synthèse}

90- Agrafe de ceinture

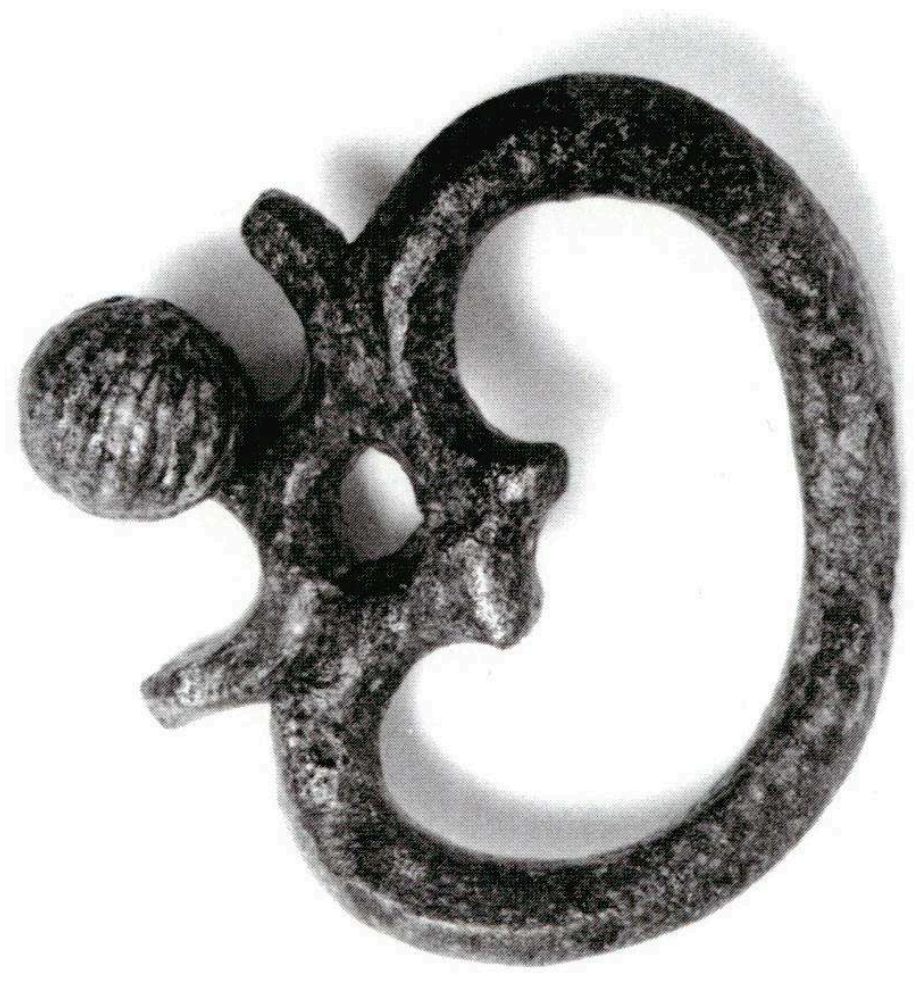

\section{La cohérence des données archéologiques}

Parvenus au terme de cet inventaire, il nous faut revenir sur la question de l'intégrité du mobilier, question fondamentale pour une découverte archéologique de 1818 qui figure parmi les plus anciennes effectuées en France. Il y a bien eu fouille au sens moderne du mot et même publication partielle des données recueillies. A la suite de la découverte, un rapport de fouille accompagné de planches figurant les objets les plus significatifs a été écrit par François Artaud, directeur du Conservatoire des Arts de 
Lyon. Publié immédiatement dans la presse de l'époque, ce texte non illustré était manifestement appelé à devenir une publication avec plusieurs planches qui, pour des raisons inconnues, ne vit jamais le jour. Les différentes versions du manuscrit, dont une seule contient les planches (collection privée) constituent un document étonnant, tant par la précision des données de terrain (dimensions du tumulus, de la fosse, des objets) que par les conclusions auxquelles parvient Fr. Artaud: le tumulus de Verna serait la tombe d'un gaulois du temps des colonies écrit-il, une interprétation qui est loin d'être erronée vu la datation ici proposée, à savoir les débuts du Ier s. av. J.-C. Autre modernisme, l'article d'Artaud contient ce qui apparaît être l'une des premières planches archéologiques de comparaison. En effet, une épée gauloise de la région de Servanes/Mouriès (Bouches-du-Rhône) est illustrée dans le manuscrit pour sa ressemblance avec celles trouvées par le comte de Verna.

2 Pourtant, les différentes versions des manuscrits posent un problème évident lorsque l'on tente l'étude d'une découverte bien antérieure à l'époque où l'archéologie française se dota de ses premiers outils méthodologiques. Le mobilier mentionné avec plus ou moins de précisions dans le texte n'est pas systématiquement illustré ; quant à la collection de Verna, elle comprend un grand nombre d'objets et de fragments qui ne figurent ni dans les manuscrits, ni dans les planches d'époque. La question de l'intégrité du mobilier est donc un préalable à tout travail de synthèse sur cet "ensemble " funéraire laténien exhumé il y a près de deux siècles. S'il a été possible de hiérarchiser le mobilier en fonction de divers critères, citations dans le texte et renvois à une illustration, citations sans illustrations, généralités, allusions, cette analyse a vite montré ses limites en l'absence d'un véritable relevé en place du mobilier.

3 Cependant, la falsification paraît exclue si l'on considère que Fr. Artaud ne semble guère avoir profité d'une découverte vite oubliée. Il s'agit certes bien d'une collection locale regroupant le produit de la fouille de deux sites, l'un campaniforme, l'autre gaulois, mais sans intrusion de mobilier extra-régional (on pourrait penser à des intrusions de mobilier provençal, mais aucun des objets donnés par A. Revoil à Fr. Artaud ne figure dans la collection). Toutefois, peut-il s'agir d'une collection composite, réunissant le fruit de plusieurs découvertes locales datant de l'âge du Fer?

4 Le problème posé par le bassin étrusque et sa chronologie décalée par rapport au reste du mobilier datable ne paraît pas insoluble. En Gaule (et ailleurs) bien d'autres exemples témoignent du dépôt d'objets remarquables longtemps après leur fabrication et l'on peut même avancer l'idée que ces "pièces de collection" sont l'une des composantes du mobilier funéraire des sépultures à biens privilégiés. Désuètes, voire inutilisables, elles n'en enrichissent pas moins les funérailles en venant augmenter le total des biens de prestige "consommés» par la cérémonie. D'un autre côté, l'intégration d'objets " antiques " peut être indirectement une façon de rappeler à tous le souvenir de leurs anciens possesseurs, vraisemblablement les ancêtres du défunt. Dans le cas de Verna, l'étude d'A.-M. Adam montre que le podanipter avait subi le même rituel que le reste du mobilier figurant dans la tombe, ce qui permet de ne pas écarter cette importation de l'ensemble restitué alors par les fouilleurs. Les choses se compliquent avec certains objets moins spectaculaires. Il y a quelques minces divergences entre les manuscrits (il y a en parfois dans des relations de fouilles bien moins anciennes que celle-ci) et elles pourraient inviter à se demander si la collection ne fut pas constituée par la réunion de plusieurs sépultures laténiennes autour d'un 
ensemble principal; le nombre de pièces d'armements, les épées notamment, permettent de l'envisager.

5 De plus, des affirmations tardives d'E. Chantre viennent semer le doute; selon lui, la fouille d'une tombe à char aurait été effectuée quelque temps avant sa venue dans l'Isle Crémieu, précisément durant l'hiver 1864. Rédigée plus de vingt ans après, la relation de cette prétendue découverte étonne dans la mesure où $\mathrm{E}$. Chantre paraît ignorer la trouvaille de 1818. L'inventaire et la description du mobilier peuvent laisser croire à la découverte d'une autre tombe à char - peut-être du tout début de l'âge du Fer - sur les terres de la famille de Verna, découverte accompagnée d'autres tombes laténiennes, éventuellement des tombes adventices.

6 Enfin la possibilité selon laquelle plusieurs tombes auraient été découvertes et immédiatement mélangées à une sépulture centrale ne pourra jamais être totalement écartée, mais il faut rappeler la qualité de la fouille telle qu'elle nous apparait dans les relations et affirmations des auteurs. Outre les pièces intactes ou quasi-intactes, des fragments, parfois totalement déformés par le feu, mesurant quelques centimètres ont été patiemment recueillis; la restauration et l'étude ont montré qu'ils appartenaient bien aux vases décrits et présentés comme provenant d'une seule et même fosse. De même, une partie des ossements (animaux et humains) a été récupérée, ce qui apparaît très précurseur.

7 Enfin, quelques disparitions d'objets restent assez énigmatiques, comme celle du casque identifié lors de l'étude initiale. Si l'on peut penser à un cadeau fait par l'inventeur à quelques collectionneurs prestigieux de son époque, rien n'explique pourquoi la planche figurant l'objet est absente, tant chez les héritiers actuels que dans les archives de l'Académie de Belles Lettres... Certains objets ont pu disparaître des vitrines dans lesquelles ils étaient exposés chez les propriétaires. C'est le cas d'une figurine exceptionnelle à l'âge du Fer représentant une grenouille, photographiée en 1986 ; on rappellera que dans le produit des fouilles d'E. Chantre près du Camp de Larina figuraient deux objets annulaires en bronze, stylistiquement datables de La Tène, ornés d'oiseaux et de grenouilles (Perrin 1990 : 15 et note 3).

8 Inversement, "l'ensemble " de Verna pourrait avoir été plus important que ne le montre l'inventaire ici donné. En effet, dans les réserves de la Maison du Patrimoine figurent aujourd'hui plusieurs tôles de bronze, sans provenance précise, appartenant à des récipients dont l'aspect évoque très fortement celui des treize vases attribués à la tombe.

9 En conclusion, des doutes de principe subsistent et subsisteront tant qu'une tombe de même ampleur n'aura été découverte en Dauphiné.

\section{Interprétation du dépôt à la lumière des connaissances modernes : sépulture ou dépôt ?}

10 Compte-tenu des réserves exprimées, tout en considérant comme probable l'homogénéité d'une large partie du mobilier, il semble raisonnable de limiter la synthèse à quelques observations.

11 Certains travaux et découvertes récentes de "tombes » laténiennes et gallo-romaines sans défunt (Boulestin, Buisson, Gomez de Soto 2002) invitent à s'assurer de la nature réelle du site fouillé par le comte de Verna. À quel type de site les inventeurs eurent-ils 
affaire : sépulture comme ils le pensaient ou dépôt cultuel ? La première observation remonte à la fouille. En effet, il est précisé que le mobilier témoigne en partie d'une crémation plus ou moins prolongée, ce que les examens modernes confirment totalement. L'hypothèse d'une sépulture repose sur un unique fragment osseux, quelques millimètres d'une côte "vraisemblablement » humaine manifestement brûlée et soudée au bord du podanipter ce qui explique sa conservation. C'est très peu pour affirmer qu'il s'agit bien d'une tombe, mais on soulignera que bien des crémations laténiennes, parfois fouillées par des anthropologues, ne livrent que des "pincées " d'ossements prélevées dans les cendres du bûcher. Quel type de crémation? La tombe était-elle du type bustum (primaire) ou autre? Aucune rubéfaction des parois n'est signalée, mais la quantité de petits fragments d'objets plaide en faveur de ce mode de crémation (il y aurait eu environ $2 \mathrm{~m}^{3}$ de cendres, charbons, os et objets).

D'autres restes humains devaient être présents dans la sépulture, mais sans doute étaient-ils peu nombreux et très fragmentés; en effet, les fouilleurs ont recueilli des ossements brûlés d'un cheval et d'un porc mesurant quelques centimètres; ils auraient certainement fait de même pour des fragments de crâne ou autres restes humains subsistant généralement à la crémation. En définitive, l'ensemble dit de Verna semble bien être une sépulture, mais, nonobstant les remarques faites plus haut, s'agit-il d'une tombe individuelle? Le nombre des armes, des épées (5) et des boucliers (3) notamment, va à l'encontre de l'usage le plus commun à l'âge du Fer, qui dans le cas de la sépulture individuelle, est celui du dépôt d'un seul équipement, notamment d'une seule épée, d'un seul bouclier, mais d'une à plusieurs lances. Cependant, on le sait, des cas très particuliers existent à l'image de l'ensemble de la Mailleraye-sur-Seine. L'idée d'un unique personnage au rang supérieur à d'éventuels accompagnateurs est illustrée par la mention du casque ; peut-être pouvait-il outre sa panoplie principale posséder des équipements complémentaires. Mais ces panoplies de guerre apparemment surnuméraires pourraient très bien appartenir à des défunts secondaires, brûlés en même temps, à moins qu'elles ne représentent symboliquement des individus physiquement absents des funérailles. Comme on le voit, on touche là aux limites de la documentation: tout au plus peut-on affirmer qu'il y a eu crémation d'au moins un défunt.

\section{Sélection et traitement du mobilier déposé dans la tombe}

Dans l'hypothèse d'un ensemble homogène, il convient d'expliquer la concentration exceptionnelle du mobilier. De plus, la restauration des objets a permis de mettre en évidence divers types de traitements du mobilier sélectionné avant les funérailles puis à l'issue de la crémation pour accompagner le défunt dans l'au-delà : coups, torsions et pliages, démontages. Alors que les épées et les boucliers témoignent de manipulations destructrices, les lances ont seulement été placées sur le bûcher funéraire; aucune information ne vient préciser le traitement du casque. La vaisselle métallique républicaine - des vases destinés aux ablutions d'avant banquet et au service du vin est non seulement exceptionnelle par son abondance, mais aussi par la présence de types originaux ou dotés de caractères peu ordinaires. Ainsi, l'étude des dimensions des différents récipients montre que les vases accompagnant le défunt sont tous plus grands que ceux habituellement rencontrés. Le statut du défunt explique assurément 
cette sélection, mais aussi la présence d'un unicum (bassin rectangulaire) et de formes très rares (couvercle d'un vase de forme inconnue, bassin à bec verseur). Comme une partie des armes, certains de ces vases en bronze ont fait l'objet d'une forme de sacrifice conduisant à leur destruction. Quant au char, en partie brûlé, il est l'une des rares attestations archéologiques d'un de ces véhicules à quatre roues de la fin de l'âge du Fer dont le nom nous est connu en gaulois. Ce véhicule de parade dont la caisse et les roues étaient ornées, témoigne d'influences romaines en particulier pour ce qui concerne la construction de l'essieu tournant à pivot en fer et l'ornementation des tiges.

14 Cette synthèse entre techniques de charronnage celte et romain s'explique aisément vues la position géographique de la découverte et sa datation. Deux chevaux ont tiré le char par un joug dont seuls quelques éléments ont été conservés. L'attelage était dirigé à l'aide d'une paire de mors de bride de tradition italique. Quant au troisième mors de bride, il a vraisemblablement été déposé pour symboliser la possession d'un cheval monté, voire l'appartenance à une élite cavalière. Cette combinaison de trois mors existe dans plusieurs autres tombes à char, ainsi que dans les tombes à char à deux roues, souvent avec une paire de mors de filet et un mors de bride. Enfin, la présence d'une faucille intacte en fer peut se comprendre comme l'affirmation d'une des sources de la richesse du défunt, des dépôts comparables se rencontrant dans les tombes aristocratiques des Bituriges et des Trévires (outils de forgerons ou meules).

\section{Datation de la tombe}

Déjà en 1818, Fr. Artaud s'étonnait de n'avoir pas découvert de monnaies (autre indication sur l'attention portée à la fouille), ce qui l'aurait certainement aidé à préciser l'époque à laquelle vécut «ce Gaulois du temps des colonies romaines ». Parures vestimentaires, céramiques importées, vaisselles et certains aspects technologiques fournissent les dates les plus précises. Tout d'abord, notons les fibules essentiellement du type "à pied solidaire de l'arc", un mode de fabrication apparu vers le deuxième quart du IIIe s. av. J.-C., «abandonné » au Ier s. av. J.-C., puis «redécouvert » sous les principats d'Auguste et Tibère. La vaisselle à vernis noir, semble-t-il d'origine campanienne, de mauvaise qualité, usée et pourtant réparée (un cas), et la vaisselle métallique permettent également d'avancer une datation au cours de la phase La Tène Dlb.

Ainsi, cet ensevelissement aurait eu lieu aux alentours des années 100-80 av. J.-C., après la conquête de la Transalpine, voire après le passage des Cimbres en Gaule - deux événements qui ont nécessairement eu des implications au niveau local - et peu avant le passage de Pompée en route pour l'Espagne qui affecta, semble-t-il, d'une autre manière les élites locales (Tarpin 2002). Historiquement, le personnage enseveli dans l'un des tumulus de la bordure occidentale de l'Isle Crémieu fut très probablement un des contemporains d'Indutiomaros, d'Abducillos et de Catugnatos, notables allobroges mentionnés lors d'événements des années 60 av. J.-C. Le mobilier funéraire affirme qu'il s'agissait d'un adulte masculin, sans qu'il soit possible d'avancer un âge quelconque ; peut-être naquit-il avant la conquête de $121 \mathrm{av}$. J.-C. Le défunt vécut donc au moment où le Camp de Larina était occupé, cette fréquentation générant une partie du mobilier déposé dans la faille de la Chuire ; il est aussi semble-t-il contemporain des dépositions funéraires et de l'habitat d'Optevoz sur le plateau de Crémieu, de la nécropole de 
Mépieu et surtout de l'occupation pré-romaine toute proche du domaine du Vernai à Saint-Romain-de-Jalionas dont la chronologie laténienne reste à préciser.

\section{La tombe de Verna parmi les sépultures aristocratiques de la fin de l'âge du Fer}

17 La tombe de Verna est un ensemble à mobilier privilégié, ne serait-ce que par le nombre et la qualité de la vaisselle importée qui y figure. Le dépôt d'autant de biens de valeur, coûteux tant dans le monde gaulois que dans le monde romain, caractérise un personnage de rang social élevé, membre d'une classe aristocratique numériquement faible : à peine $3 \%$ de la population libre selon des données extrapolées d'après TiteLive à propos des Boïens d'Italie au début du IIe s. av. J.-C. (Perrin, Decourt 2002). La comparaison avec les quelques autres ensembles «riches " montre que la tombe de Verna, compte-tenu des réserves exprimées plus haut, paraît être l'une des plus importantes pour l'époque considérée. En fait, il est vraisemblable que des tombes bien plus importantes, comparables à celles des rois Bretons, restent à découvrir.

Le rituel de cette tombe allobroge évoque fortement un passage célèbre figurant dans la Guerre des Gaules (éd. L. - A. Contans, CUF), précisément dans l'excursus ethnographique (livre VI). César l'a en partie emprunté (et donc traduit) des Histoires de Poséidonios d'Apamée, ce philosophe grec qui parcourut la Gaule méridionale vers 101-100 av. J.-C., voyageant probablement de Massalia vers la nouvelle Narbo Martius (Narbonne), avec l'ambition de poursuivre les Histoires de Polybe terminées en 146 av. J.-C. Son oeuvre perdue traitait de la conquête de la Transalpine, conquête impliquant au premier chef les Allobroges, et de la migration des Cimbres et des Teutons. Dans le tableau descriptif de la société gauloise conservé par César figurent des passages sur les croyances des Gaulois et la manière dont ils traitent leurs défunts.

«Les funérailles sont, relativement au degré de civilisation des Gaulois, magnifiques et somptueuses ; tout ce que, penset-on, le mort chérissait est porté au bûcher, même les animaux, et il n'y a pas si longtemps encore, la règle d'une cérémonie funèbre complète voulait que les esclaves et les clients qui lui avaient été chers fussent brûlés avec lui ».

Le jugement qui débute ce célèbre texte concerne sans doute des pratiques funèbres de la deuxième moitié du IIe s. av. J.-C., mais César en reprenant Poséidonios témoigne du maintien de rites somptuaires dans la première moitié du Ier s. av. J.-C. De plus, le rituel décrit est clairement une évolution (une simplification en fait) de pratiques plus anciennes remontant peut-être aux débuts du IIe s. av. J.-C., voire au IIIe s. av. J.-C. L'archéologie montre que ces funérailles ne sont manifestement pas communes à tous les Gaulois: il s'agit de crémations particulières, réservées aux membres de l'aristocratie gauloise, peut-être celle des peuples directement concernés par l'ouvrage de Poséidonios. L'auteur révèle un déroulement qui s'oppose aux usages grécoromains : ces funérailles sont en effet "magnifiques et somptueuses", c'est-à-dire spectaculaires et coûteuses, consommatrices de biens de valeur. Pourtant, et c'est là quasiment une règle dans le monde gaulois, l'orfèvrerie et l'argenterie ne sont pratiquement jamais déposées dans les bûchers ou dans les tombes. La dépense est ailleurs, sans doute dans la destruction et l'abandon de mobiliers de prestige, l'abattage d'animaux sacrifiés pour le banquet, la consommation de boissons importées et la distribution de richesses diverses. 

fusionne les informations transmises par César, Pomponius Mela et par l'auteur des Schlolies à la Pharscale de Lucain, un modèle littéraire de la cérémonie funèbre aristocratique gauloise apparaît. Le rituel concerne un homme adulte, un guerrier de rang social élevé, membre de l'aristocratie, père de famille. Il sera enseveli avec beaucoup d'objets lui appartenant personnellement; au cours de la cérémonie des sacrifices d'animaux, notamment de chevaux auront lieu. Enfin, certains proches du défunt, esclaves, serviteurs, clients, épouses et concubines l'accompagneront dans l'audelà, grâce au feu du bûcher, médiateur entre le monde des vivants et celui des morts dans lequel séjournera un temps seulement le défunt.

21 De toutes les sépultures à mobilier privilégié actuellement connues, celle de Verna est sans doute la plus proche du rituel tel qu'il apparaît dans la synthèse des textes antiques. Compte-tenu de la localisation de la tombe, en Province de Transalpine, à 300 $\mathrm{km}$ de Massalia et près d'un axe aisé de circulation, il est tentant de voir dans cette découverte l'expression matérielle des laconiques descriptions gréco-latines. Cependant les différences avec le modèle envisagé sont finalement assez nombreuses : ainsi, à Verna, la crémation du mobilier est partielle et non totale comme l'affirment les textes. En revanche, la destruction d'une nombreuse vaisselle en bronze est quasi systématique, rite sur lequel les sources sont muettes. Alors qu'à Verna, un abattage d'animaux domestiques peut être mis en évidence, rien ne vient alimenter l'idée d'un sacrifice massif ; cela tient sans doute aux choix opérés sur le terrain. Le sacrifice des chevaux logiquement présents lors de la cérémonie et leur dépôt par-dessus la tombe n'est qu'une hypothèse invérifiable. Du vin italien d'origine étrusque a été consommé ou utilisé pour éteindre le bûcher, mais seuls deux fragments de conteneur passé par le bûcher ont été conservés. C'est peu pour une sépulture d'une telle importance, mais aucune des sépultures allobroges de cette époque ne contenait d'amphores, entières ou à l'état de fragments. Peut-être les amphores ont elles été écartées de la fosse funéraire. Enfin, l'existence de plusieurs défunts - doryphores ou clients - n'est qu'une hypothèse que l'on pourrait avancer à partir du nombre des épées, des boucliers et des lances ; les fragments de crânes non brûlés étant intrusifs.

La tombe allobroge de Verna se distingue également de l'ensemble des sépultures riches de La Tène finale, mais des points communs existent ici et là. Le char n'est pas symboliquement représenté par un de ses éléments métalliques, mais est au contraire complet; sans doute a-t-il été placé au-dessus de la fosse comme lit funéraire. La présence de ce véhicule, sans doute ce que les Gaulois nommaient petorritum, témoigne d'une volonté de renouer avec une longue tradition remontant à l'âge du Bronze, tradition peut-être locale si l'on considère la pièce de char du Hallstatt B3/C des collections de la Mairie de Crémieu. Le traitement par le passage sur le bûcher est en revanche bien une pratique de la fin de l'âge du Fer. On note qu'il n'y a pas à proprement parler d'urne cinéraire, bien que le seul fragment d'os humain préservé soit effectivement fixé sur le bord externe du plus spectaculaire des vases de bronze, le seul qui soit décoré. L'antique podanipter semble avoir été brisé avant la crémation ce qui, sans l'interdire totalement, complique l'hypothèse de son emploi comme réceptacle des restes incinérés du défunt.

Outre la présence d'une "antiquité ", observation fréquente au sein des tombes des élites hallstattiennes, la volonté de s'aligner sur des usages anciens s'exprime par l'emplacement de la découverte effectuée dans un tumulus, haut de près de $4,50 \mathrm{~m}$ si on en croit Fr. Artaud; ce type de construction funéraire est également inhabituel à la fin 
de l'âge du Fer, même si dans plusieurs régions de Gaule l'hypothèse de tertres peu élevés établis par-dessus des tombes riches et aujourd'hui arasés est une certitude (Lambot 2002). Cependant, il faut reconnaître que l'immense majorité des milliers de tumulus fouillés depuis le XIXe s. appartient bien au premier âge du Fer et à l'âge du Bronze ; statistiquement, on devrait disposer de découvertes contemporaines à Verna, ce qui n'est pas le cas. La question se pose de savoir si le tumulus a été érigé pour cette sépulture fastueuse ou si la tombe laténienne a réutilisé un tertre plus ancien appartenant à la fin de l'âge du Bronze et au début de l'âge du Fer. Seule la localisation $\mathrm{du}$ tumulus en question et la reprise des fouilles permettraient de le savoir. Pour l'heure cette tombe à char gauloise apparaît isolée au sein d'une nécropole établie environ sept siècles auparavant et comprenant au moins une tombe aristocratique des années 800 av. J.-C. (et peut-être deux).

Ce personnage de haut rang témoigne d'un conservatisme bien particulier, à un moment où les Allobroges soumis sont intégrés à la Provincia. Tous les archaïsmes observés ou suspectés (tumulus, char, dépôt en vase métallique ?) pourraient laisser penser que le fameux passage de Poséidonios est bien incomplet, notamment sur les formes que pouvait prendre le rite traditionnel, cette "cérémonie funèbre complète " sur laquelle l'auteur ne retient que la multiplicité des défunts sacrifiés ou volontaires pour accompagner le défunt principal. Pourtant, l'idée d'une influence romaine ne peut être écartée. On regrettera d'autant la disparition du casque en bronze «simple » et "inorné» qui pourrait avoir été de type romain plutôt que gaulois. L'aristocrate enseveli, rappelons-le, près du site de Larina et de l'occupation du site du Vernai à Saint-Romain, occupait un emplacement stratégique à la frontière des Allobroges et par conséquence sur la limite septentrionale de la Province. Des liens avec le pouvoir romain ou avec les négociants romains et grecs expliqueraient l'opulence dont il bénéficia de son vivant et qu'il manifesta jusque dans la mort. Strabon (d'après Poséidonios) attribue précisément à la noblesse la transformation de Vienna, simple village mais capitale-mère des Allobroges, en cité (polis) (Lucas 2002) ; Verna étant situé à plus de quarante kilomètres de Vienne, il n'est pas sûr que le défunt ait été l'un des membres de cette noblesse viennoise urbaine pouvant résider à la campagne; en revanche rien n'interdit de penser qu'il ait pu faire partie du sénat allobroge qui devait se rassembler à Vienne.

A ce jour, la question de sa résidence reste ouverte : le site fortifié du Camp de Larina ? Le domaine de Saint-Romain? A n'en pas douter, il y a là un terrain de recherches particulièrement intéressant pour l'analyse des élites de la fin de l'âge du Fer. 


\title{
Annexes
}

\author{
Catherine Bellon et Stéphane Carrara
}

\section{Annexe 1. Transcription des manuscrits des archives}

Il s'agit ici d'un choix effectué parmi les deux manuscrits de l'Académie des Sciences, Belles Lettres et Arts de Lyon (Ms 101/3 et Ms 357) et les deux de la collection privée. C'est en fait le même texte, recopié et simplifié pour la publication dans «Le Moniteur Universel ", possédant parfois des indications supplémentaires (appels de notes, commentaires, planches, mesures des objets). Les planches mentionnées ne sont aujourd'hui présentes que dans la collection privée alors que deux (III et V) ont été reprises à la fin du Manuscrit de Lyon souterrain de Fr. Artaud (Ms 104), augmenté d'une nouvelle gravure des quatre tumulus ( $c f .4 \mathrm{e}$ de couverture).

\section{Notice extraite du Moniteur Universel du 24 décembre 1818 (non signée) (collection privée)}

2 Un ami des arts distingué par ses connaissances, transmet de Lyon les détails suivants sur les objets curieux trouvés dans un tombeau antique. Ce tombeau est un de ces monuments en forme de monticule connu sous le nom de tumuli, dont on trouve encore un assez bon nombre dans les diverses contrées de la France. Celui-ci est situé à Jalionas, département de l'isère, près de St Romain, à peu de distance du Rhône et du château de Verna. Son ouverture a fait trouver d'abord des ossements, des cendres, des charbons, et plusieurs armes et ustensiles de diverses sortes. Les plus remarquables de ces objets sont un casque de cuivre sans ornement, deux longues épées de fer très minces et à deux tranchants, dont l'une, fixée dans son fourreau, est repliée en trois parties ; un grand vase ou espèce de marmite en cuivre battu et tourné, une aiguière à anse avec son couvercle de style romain, des fragments de vase ou casserole de cuivre jaune et de poterie grossière de fabrique gallo-romaine; des boucles, fibules et autres ornements, dont un a la forme d'une grenouille de grandeur naturelle ; des mors en fer, fabriqués de manière à serrer à la volonté du cavalier, la bouche du cheval ; des pièces de harnais. 
3 Parmi toutes ces ferrailles, ce qui est plus curieux et plus digne d'examen, c'est la ferrure aisée à reconnaître d'un char, laquelle paraît complète. Les quatre moyeux sont en bronze, avec deux rainures extérieures; les clavettes qui retenaient les roues sont ornées; les bandes de ces roues sont en fer : on distingue aussi les accoudoirs du char. Une faux de fer, arrondie comme une serpe, paraît y avoir été attachée pour l'armer; cette faux est en effet percée de quelques trous à l'extrémité, qui a du tenir au char, et les clous sont restés dans ces trous. On croit qu'il ne serait pas impossible, d'après toutes ces parties, de former un dessin assez exact d'un char et d'un attelage antique.

4 Tous ces objets ont été recueillis avec soin par M. de Verna, amateur éclairé des arts, qui se propose de faire ouvrir d'autres tombeaux semblables voisins du premier et qu'on appelle dans le pays les quatre Molards.

5 Celui qui vient d'être fouillé, d'après ce qu'il contenait, paraît avoir renfermé un guerrier gaulois qui a vécu à l'époque des colonies romaines, et qui aura été enseveli avec ses armes et les principaux meubles à son usage. Nous aurions cru qu'on y aurait trouvé des monnaies ou des médailles ; mais il n'en est point question. Peut-être sera-ton plus heureux dans les autres fouilles.

6 On peut remarquer qu'il existe beaucoup de Molards dans le Dauphiné. On en voit plusieurs aux environs de Villeurbanne, près de Lyon, vers les anciennes Balmes viennoises; le Docteur Spon en a remarqué un très apparent entre Vienne et St Symphorien. II est possible que la fouille dont nous venons de parler détermine à poursuivre ces opérations intéressantes pour les antiquaires et les artistes.

Commentaire ajouté probablement par l'inventeur, M. de Verna (non signé) :

8 L'on doit ajouter à cette notice insérée dans le moniteur par M. Artaud Directeur du Musée de Lyon quelques détails oubliés, qui peuvent aider à faire découvrir l'époque, où le guerrier gaulois a du être enseveli dans le monticule. Un lit de charbon et d'ossement en partie brûlé, d'un pied et demi d'épaisseur, sur six en carré, prouve, à n'en pouvoir douter, qu'à la dite époque, l'on était en usage de brûler les corps, et le feu même a du être si violent, que l'on a trouvé quelques parties des ornements du char et de l'armure entièrement fondues; à cette observation, on peut joindre celle, que les trois sabres que cette fouille a produite, étaient tous repliés en trois parties, et aux fragments de leurs fourreaux on a pu reconnaître que deux de ces fourreaux étaient en fer, et l'autre en cuivre. Les roues trouvées sont au nombre de quatre, ce qui semble indiquer un attelage de quatre chevaux ; un d'eux a été le sujet d'un examen particulier de MM. les professeurs de l'école vétérinaire de Lyon qui ont du en faire un rapport à la Société d'Agriculture de cette ville.

\section{Manuscrit de Fr. Artaud à M. de Verna (avec les planches I, III, IV, V, non signé) (collection privée)}

9 Des objets curieux viennent d'être trouvés dans un tombeau antique situé à Jalionas département de L'isère arrondissement de Crémieux près du Château de Verna. Ce Tombeau est de ces monuments en forme de Tumuli dont on trouve encore un assez bon nombre dans les diverses contrées de la France. Son ouverture a fait rencontrer d'abord des ossements, des cendres, des charbons et plusieurs armes et ustensiles de diverses sortes. Les plus remarquables de ces objets sont un casque de cuivre sans ornement (pl. IIP.), deux longues épées de fer très minces et a deux tranchants, dont 
l'une fixée dans son fourreau est repliée en trois parties (pl. III.BB.AA.), un grand vase ou espèce de marmite en cuivre battu et tourné (pl. I.A.), une aiguière à anse avec son couvercle de style romain (pl. III.EE.), des fragments de vases ou casseroles de cuivre jaune et de poteries grossières de fabrique gallo-romaine (pl. I.O.) (1), des boucles fibules et autres ornements, dont un a la forme d'une grenouille de grandeur naturelle (pl. II.M N.), des mors en fer () fabriqués de manière à serrer à la volonté du cavalier la bouche du cheval (pl. III. II.), des pièces de harnais etc.. Parmi toutes ces ferrailles ce qui est plus curieux, plus digne d'examen c'est la ferrure aisée à reconnaître d'un char, laquelle paraît complète ; les quatre moyeux sont en bronze (pl. II.Z) avec des rainures extérieures. Les clavettes qui retenaient les roues sont ornées (pl. H.X.), les bandes de ces roues sont en fer (pl. III.C.C.). On distingue aussi les accoudoirs du char (pl. U.K.). Une faux de fer arrondie comme une serpe paraît y avoir été attachée pour l'armer; cette faux est en effet percée de quelques trous à l'extrémité qui a dû tenir au char, et les clous sont restés dans ces trous (pl. II.I.). On croit qu'il ne serait pas impossible d'après toutes ces parties de former un dessin assez exact d'un char et d'un attelage antique (v. pl. V.). Strabon en parlant des peuples de la grande Bretagne dit qu'ils combattaient sur des chars ainsi que les peuples de la Gaule, c'étaient Les Efsedarii [sic !] nommés par César qui vraisemblablement avaient retenu cet usage des Troyens. Le même Strabon nous dit que l'armure des Gaulois était proportionnée à leur taille, un long sabre leur pend au côté droit (v. pl. III.AA.), leurs boucliers sont aussi forts longs et leurs lames à proportion (v. même pl. DD), ils portent de plus une espèce de pique qu'on appelle Mataris et quelques uns font usage de l'arc et de la fronde etc..

10 Tous les objets trouvés dans le Tumulus Gaulois ont été recueillis avec soin par M. de Verna amateur éclairé des arts qui se propose de faire ouvrir d'autres tombeaux semblables voisins du premier qu'on appelle dans le pays les quatre Molards. Celui qui vient d'être fouillé d'après ce qu'il contenait parait avoir renfermé un guerrier Gaulois qui a vécu à l'époque des colonies romaines () et qui aura été enseveli avec ses armes et les principaux meubles à son usage. Ce qui nous fait pencher pour cette idée, c'est que la forme des objets qui ont été trouvés dans son tombeau sont de style Romain et qu'un débris de vase semblable à celui de la planche I. lettre G. a été trouvé récemment dans les ruines d'une habitation romaine, chez $\mathrm{M}$. Nugues président à la cour royale de Lyon dans le territoire de Pian (?) près de Bourgoin département de l'isère. D'autre part $M$. Revoil directeur de la poste aux lettres à Aix a découvert dans sa terre de Servanne une longue épée en fer fort mince avec son fourreau (v. pl. III.KK.) semblable à celle qui a été trouvée dans le Tumulus Gaulois (v. même pl. lettre BB.). Nous aurions cru qu'on aurait rencontré des monnaies ou des médailles dans ce tombeau mais il n'en est point question peut-être sera-t-on plus heureux dans les autres fouilles.

11 On sait que l'usage d'élever de la terre sur les sépultures est d'une haute antiquité, Amyntar entre autre dans son itinéraire rapporte suivant Athenée livre XI. tome IV. qu'on voyait devant les murs de Ninive un rempart de terre élevé par Cyrus, et qu'il avait pris d'un tertre très haut qu'il fit raser lorsqu'il assiégea cette ville; ce tertre selon Amyntar était le monument même de Sardanaple et l'on y avait dressé une colonne avec une inscription Caldéenne (digne de la Secte d'Epicure).

Le traducteur d'Athénée ajoute qu'on a trouvé en Amérique cet usage d'élever des tertres ou buttes de terre sur les tombeaux et que Semiramis en avait fait élever ainsi sur ceux de ses amants qu'elle avait fait égorger. Ce sont ces sortes de monuments qui ont reçu parmi nous le nom de Molards. On peut remarquer qu'il en existe beaucoup 
dans le Dauphiné ; on en voit plusieurs aux environs de Villeurbanne près de Lyon vers les anciennes Balmes Viennoises. Le Docteur Spon en a remarqué un très apparent entre Vienne et St Symphorien. Il est possible que la fouille dont nous venons de parler détermine à poursuivre ces opérations intéressantes pour les Antiquaires et les Artistes.

\section{Manuscrit Ms 101/3 de l'Académie des Sciences Belles Lettres et Arts de Lyon (non signé)}

Le texte étant quasiment le même, ne sera reportée ici que la liste des planches, absentes, avec les mesures des objets, non répertoriées dans les autres manuscrits.

Planche. I :

A. Un pied, 2 pouces de hauteur sur un pied de large pour la marmite en cuivre battue et tournée

B. Bouton en cuivre un pouce de large ; tous les autres ornements en bouton sont faits de même. Celui-ci doit avoir servi à orner la bride ; voir KK, pl. IV. C. Terre cuite d'un vase avec les mamelons en relief. 2 pouces de large; Un morceau semblable a été trouvé à St Agrève, (Agrix ?) département de l'Isère près de Bourgoin chez M. Nugues. (Dessin du tesson de céramique intitulé Pl. II).

D. Ornement en bronze ; 2 pouces dans la queue ; cuivre ; autres moins conservés.

E. 2 pouces $1 / 2$ de haut. Cuivre. Il y en a d'autres moins conservés. A peut-être servi à fixer le couvercle d'un vase.

Planche. II

I. Faux en fer ; 2 pieds 2 lignes en hauteur. 1 pouce 6 lignes de surface de la lame.

K. En bronze ; 2 pieds 3 pouces de long, 5 lignes d'épaisseur ; peut-être un accoudoir.

L. Un pied 3 pouces 6 lignes de haut, bronze, peut-être un des bras du char.

M. En bronze ; 1 pouce 8 lignes de haut ; ornement en relief. 0 . En bronze ; 2 pouces de large et 2 de haut ; boucle.

P. 9 pouces 6 lignes de long sur 5 pouces de large; cuivre battu. Q. 11 pouces de haut; Cuivre.

R. Cuivre ; 10 pouces de long sur 2 pouces de large ; espèce de ressort.

S. 9 pouces 4 lignes de haut ; cuivre.

T. Cuivre ; 10 pouces 6 lignes de long 7 lignes d'épaisseur ; comme L plus petites.

V. Cuivre ; Minces ornements frappés. 6 pouces 3 sur 1 pouce 6 lignes de haut ;

X. 5 pouces de haut sur 4 pouces 6 lignes de large ; cuivre ; il y en a une autre incendiée à moitié fondue.

Y. Cuivre ; 5 pouces 10 lignes de haut sur 1 pouce de large. Il y en a d'autres plus petits.

Z. Cuivre; 5 pouces 5 lignes de diamètre; 2 pouces 2 lignes d'épaisseur; il y en a 4 semblables; moyeu. 


\section{Planche. III}

AA. Épée en fer dans son fourreau de même pliée en 3.11 pouces de long, 2 pouces de large, 1 pied 2 pouces de haut ; je soupçonne qu'elle a été renfermée dans la marmite A qui a 1 pied 2 pouces de haut et était de la même longueur que la suivante si elle était dépliée.
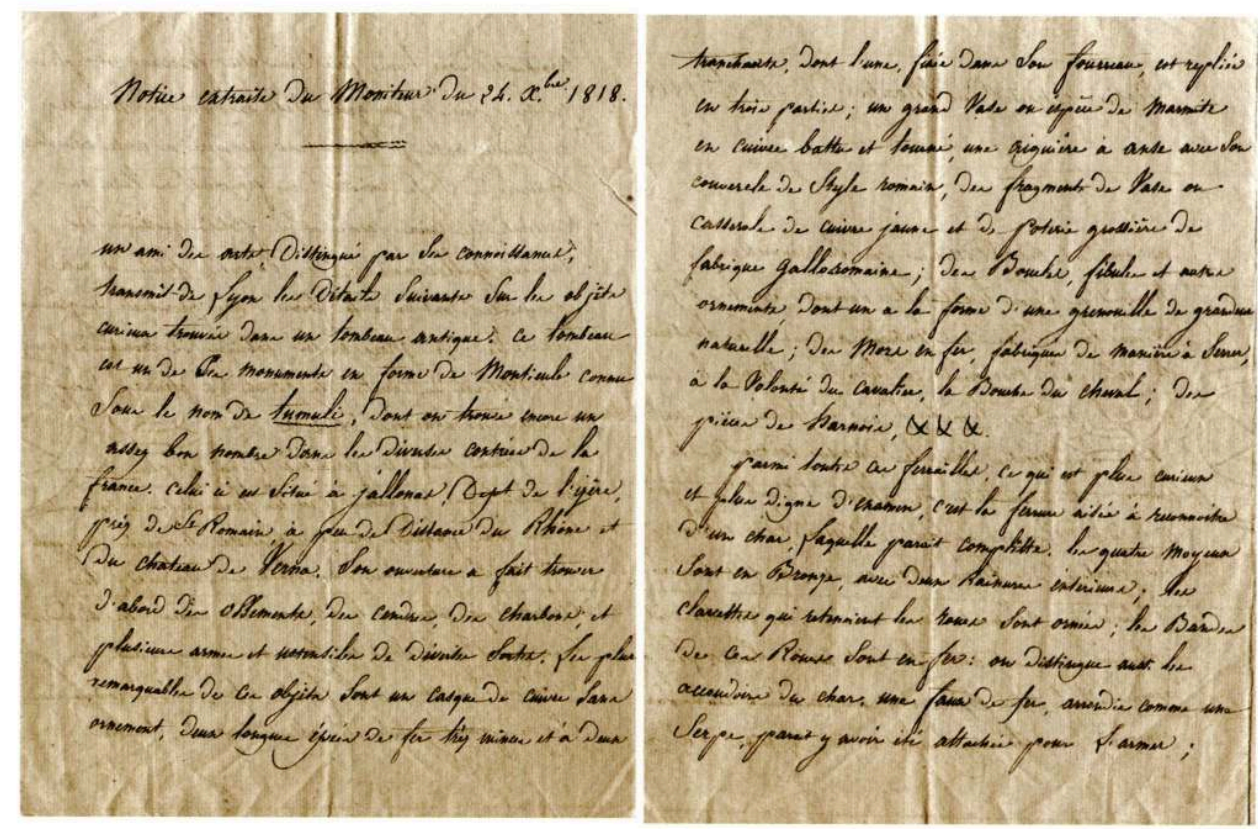


\section{Annexe 2. Nouvelles données de numismatique massaliote et celtique dans l'environnement du tumulus de Verna}

42 C'est le recueil de trois monnaies celtiques et marseillaises sur un site $^{1}$ proche de la tombe à char de Verna, au lieu-dit «Molard Renard» (I.G.N.) qui a motivé cette recherche.

43 Les inventaires de découvertes récentes et de différentes collections privées ont permis d'acquérir de nouvelles données sur la représentation des espèces monétaires celtique et marseillaise dans la région de l'Isle Crémieu et les abords du Rhône, qui viennent compléter le faciès numismatique mis en évidence par les travaux de B. Fischer (Fischer 1990 et 1991), sur le site de Larina (Isère).

44 L'étude porte sur un groupe de cinquante monnaies, dont trente proviennent du plateau de l'isle Crémieu (Isère) ${ }^{2}$ et vingt des bords du Rhône, sur le département de l'Ain $^{3}$. Ce lot monétaire se répartit entre sept monnaies massaliotes, trente-deux monnaies celtiques de Narbonnaise, et neuf de Gaule Chevelue. Deux monnaies n'ont pu être identifiées. Un élément particulier qui prend la forme d'une petite gouttelette d'argent, accompagne ce lot monétaire, il pourrait s'agir d'un flan de monnaie gauloise.

\section{L'Analyse du lot}

Trois sites distincts ont donné trois ensembles homogènes de monnaies qui pourraient appartenir à de petits lots éclatés (fig. 1).

1. Le lot de Villebois regroupe cinq monnaies au cheval galopant, un bouquetin, trois hippocampes Allobroges et une imitation des statères de Philippe II de Macédoine ${ }^{4}$.

2. Le lot de St-Vulbas présente quatre cavaliers de la vallée du Rhône, un hippocampe, un potin à la grosse tête et un quinaire du type Sequanoiotos.

3. Le lot de Faverges-de-Mépieu est constitué de huit monnaies au cavaliers de la vallée du Rhône et d'un statère imitant le monnayage de Philippe II de Macédoine. 


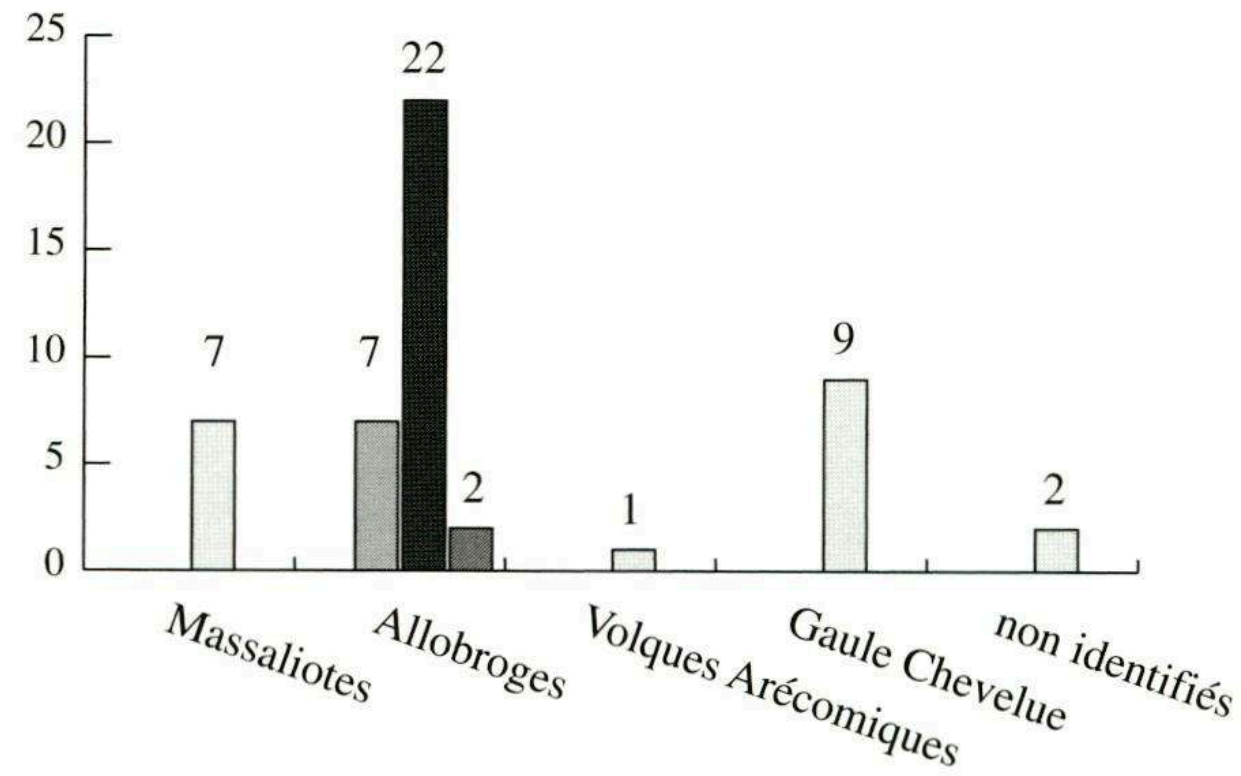

Pour les Allobroges 7 monnaies au cheval galopant et au bouquetin, 22 à I'hippocampe et cavalier du Rhône, 2 statères

La découverte de deux statères du type d'Annonay (BN 60666067), dont le profil de l'avers rappelle celui des monnaies dites Cavares au bouquetin, renforce l'idée d'un numéraire originaire de la vallée du Rhône. Bien que hors contexte, l'association de ce numéraire à des monnaies datées des environs de 90-75 av J.-C. et 75-52 av J.-C., semble confirmer la datation de la première moitié du Ier s. av. J.-C., déjà avancée pour ce type de monnayage (Deroc 1983: 24). Le style dégénérescent de ces imitations, comparativement aux exemplaires de datation plus haute, et leur poids plus léger ( 5 à 6 g.), va encore dans le sens de cette datation.

47 La prédominance des espèces monétaires allobroges parait respecter la logique qu'impose le cadre géographique de ces découvertes. Néanmoins, vu la position frontalière de la région de provenance, on aurait pu s'attendre à un faciès beaucoup moins local que celui présenté ici. Malgré tout, le lot de monnaies provenant de part et d'autre du Rhône démontre une interpénétration des monnayages entre la Gaule Chevelue et la province de Narbonnaise ${ }^{5}$ (fig. 2).

Sur la rive droite du fleuve (Ain), l'ensemble du lot monétaire provenant de cette zone, soit dix-huit monnaies identifiables, est constitué à $78 \%$ de numéraire attribuable aux Allobroges. Seules deux monnaies sont marseillaises, deux autres appartiennent à la Gaule Chevelue. Les problèmes qui persistent sur les limites du territoire allobroge et la possibilité d'une extension sur la rive droite du Rhône pourraient en partie expliquer ces chiffres. 


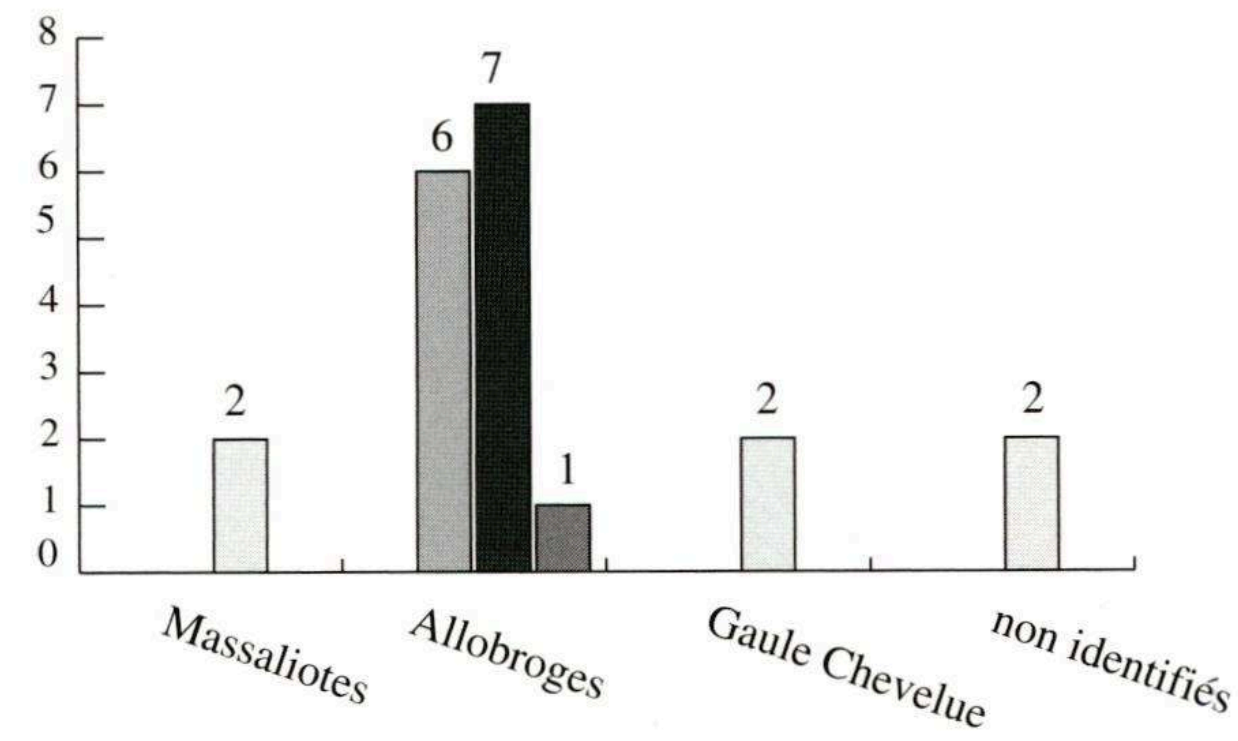

Pour les Allobroges 6 monnaies au cheval galopant et au bouquetin, 7 à l'hippocampe et cavalier du Rhône, 1 statère

Le lot provenant de l'isle Crémieu démontre la prédominance du monnayage régional, soit dix-huit monnaies sur trente, et dont quinze sont des monnaies au cavalier de la vallée du Rhône. Le reste des espèces se répartit entre cinq monnaies marseillaises et sept de la Gaule Chevelue (essentiellement de l'est et du centreest) (fig. 3).

\section{3- Répartition des monnaies de l'isle Crémieu}

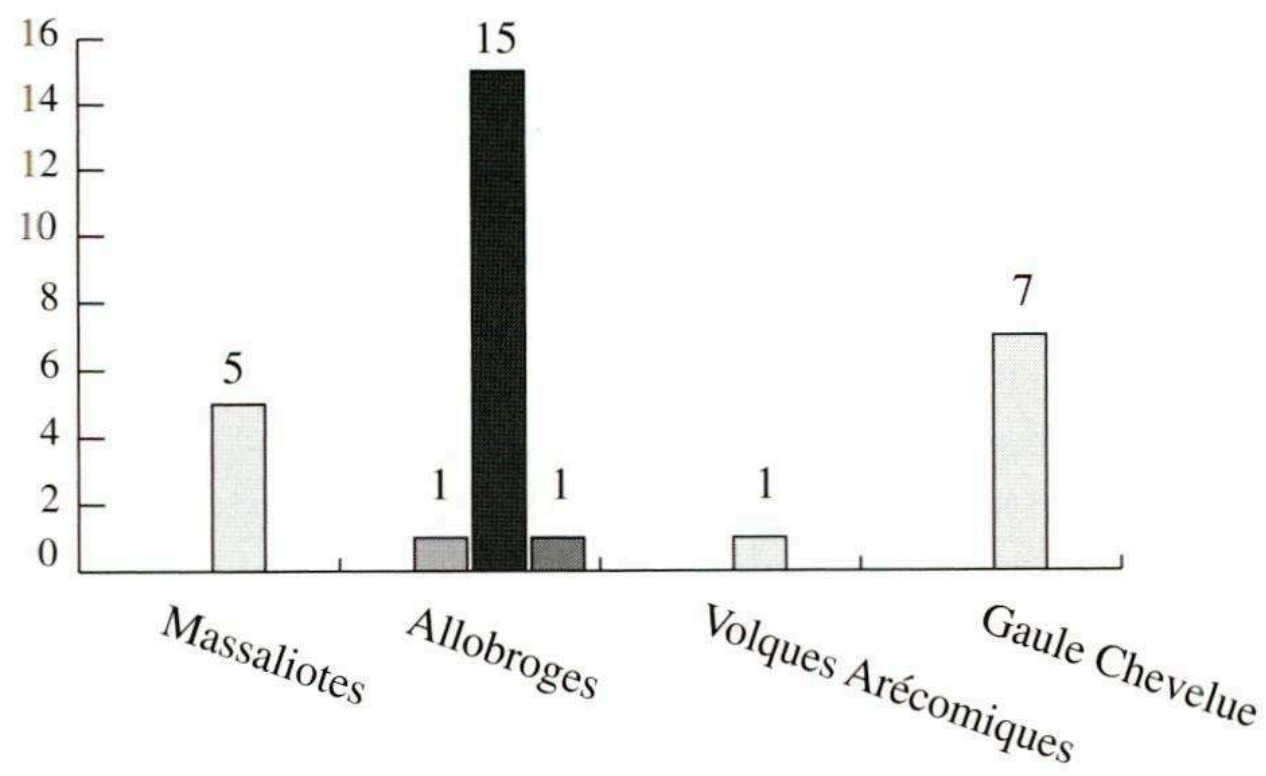

Pour les Allobroges 1 monnaie au cheval galopant et au bouquetin, 15 cavaliers du Rhône, 1 statère

Les constats faits à l'échelle du site de Larina, d'une forte représentation des espèces massaliotes et de la pauvreté des types provenant de la Gaule Chevelue (Fischer 1991), 
semblent s'inverser à l'échelle de notre lot. Sur la totalité du monnayage, $14 \%$ est d'origine (ou d'imitation) marseillaise, alors que $62 \%$ est attribué aux Allobroges ; $2 \%$ des espèces restent régionales. Le monnayage appartenant à la Gaule Chevelue est, ici, mieux représenté que celui de Marseille avec près de $18 \%$.

51 Néanmoins, l'association des monnaies étudiées par B. Fischer (1990 et 1991) et du lot présenté ici, donne un faciès local homogène, avec près de $58,8 \%$ du numéraire attribuable au monnayage allobroge ${ }^{7}$. Ceci dans une zone frontalière, sur un axe de communication non négligeable, où l'on aurait pu s'attendre à une mixité plus marquée du monnayage celtique et marseillais.

L'étude de ce lot monétaire met en évidence, dans une certaine mesure, les courants commerciaux entre Marseille, la périphérie nord de la Gaule Narbonnaise et la Gaule Chevelue $^{8}$. Le Rhône apparaît alors comme une voie et un axe de communication générant des échanges entre ces régions, et non comme une barrière les freinant (fig.

4).

4- Répartition des monnaies de Larina (Oppidum et la Chuire)

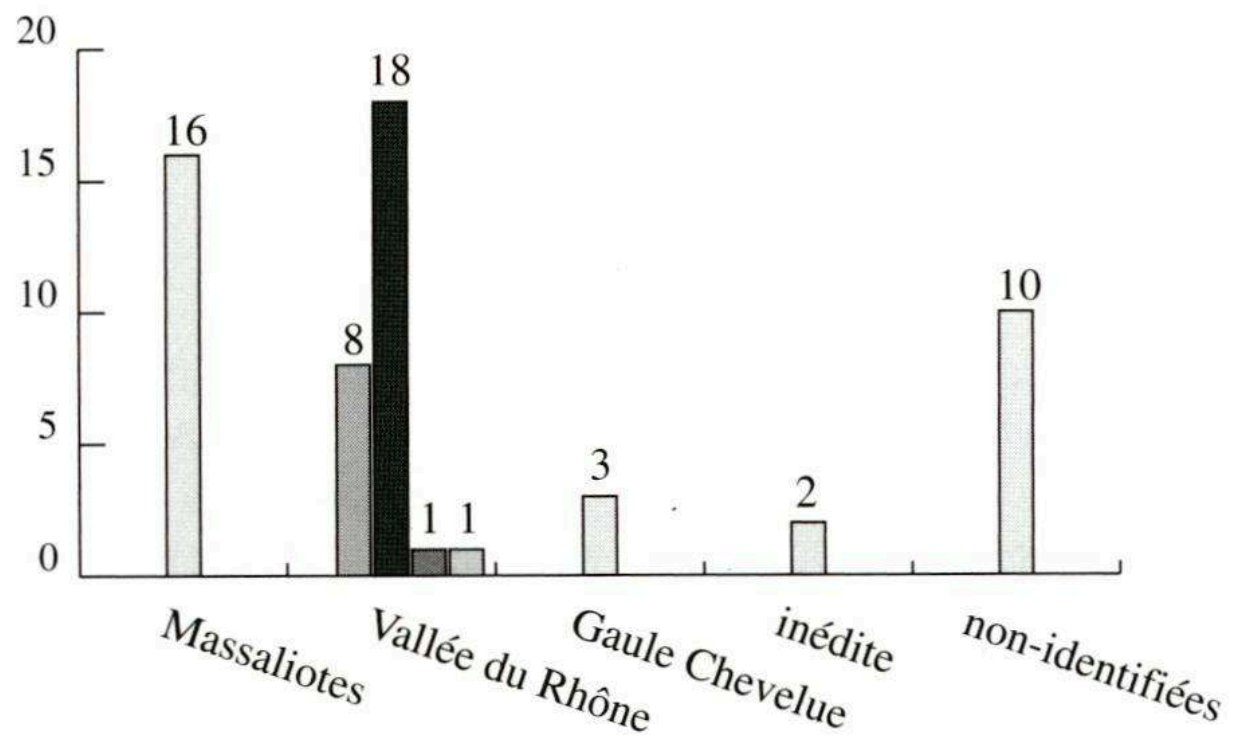

Pour la vallée du Rhône 7 Cavares, 18 Allobroges, 1 Volques Arécomiques, et 1 potin au long cou. Pour ce graphique nous avons conservé les attributions aux Cavares des monnaies au cheval galopant et au bouquetin, lors de la parution de l'article de Mme B. Fischer

Du point de vu numismatique, la découverte, en territoire allobroge, de plusieurs monnaies au cheval galopant et au bouquetin (ou cervidé) qui étaient traditionnellement attribuées aux Cavares, liées au exemplaires déjà répertoriés en $1991^{9}$, paraît confirmer une origine allobroge, aujourd'hui démontrée par la thèse de $Y$. Van Der Wielen. De plus, les statères du type d'Annonay, qui sont stylistiquement proche de ces monnayages, proviennent pour les exemplaires connus du territoire allobroge. II semble donc bel et bien s'agir d'un monnayage local. 


\section{Catalogue}

\section{Les monnaies de Marseille}

Obole de Marseille, groupe II

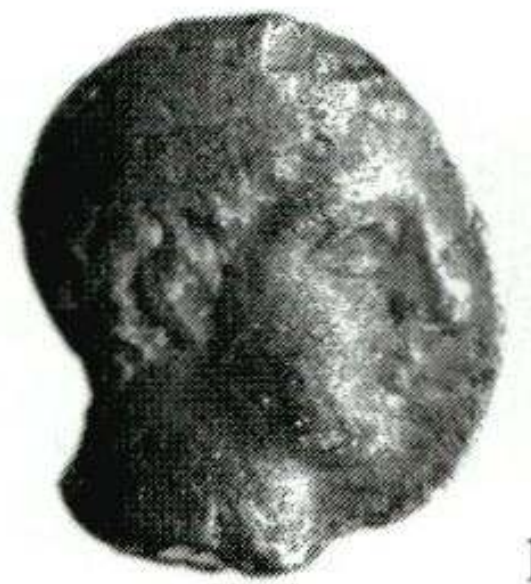

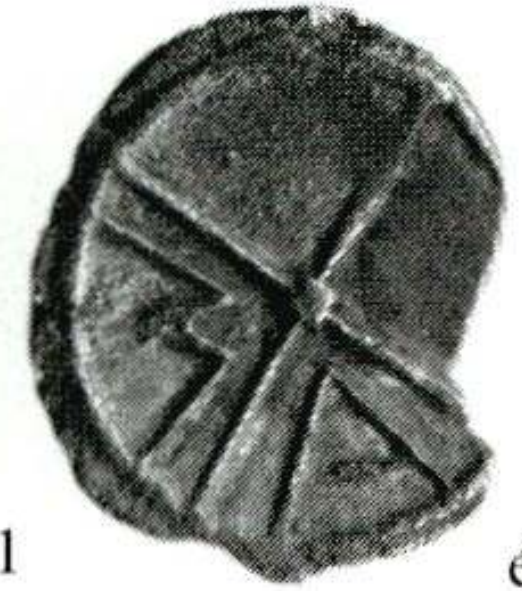

éch. 2/1

Obole de Marseille, groupe III

A/Tête à gauche, sans corne frontale ; les mèches encadrant le front et les tempes sont en forme de crochets s'étirant vers l'arrière ; devant l'oreille, de courts favoris.

$\mathrm{R} /$ Roue à quatre rayons ; M A dans deux cantons de la roue. Réf. Lyon cat. 138-144 p. 30, LT 580 pl. II.$$
\text { g. }
$$

61 3-Verna ; Argent frappé ; $10 \mathrm{~mm} ; 0,73 \mathrm{~g}$. 


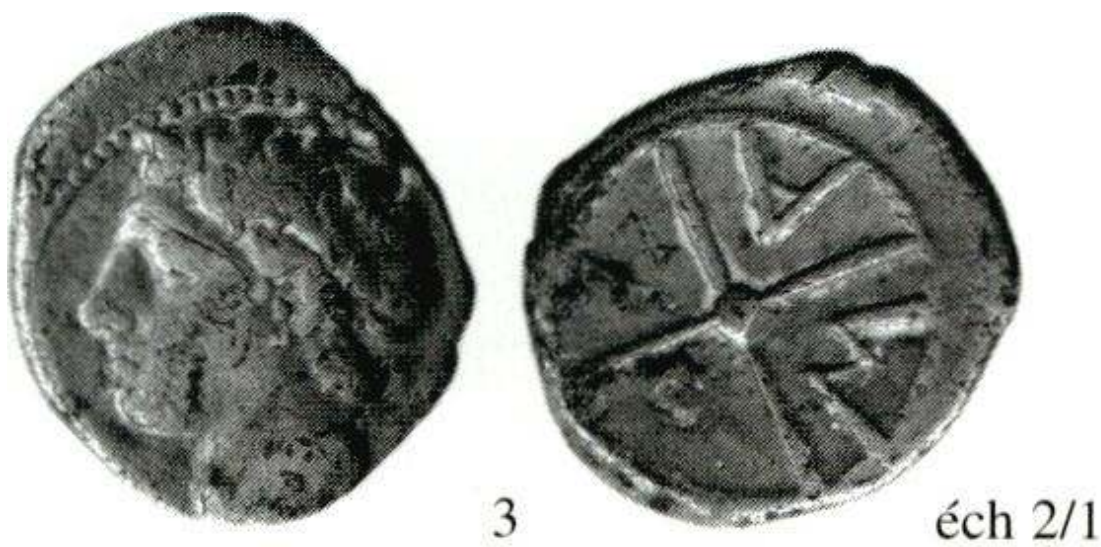

Obole de Marseille, groupe IV, émise entre 90 et 49 av. J.-C. (imitation locale ?)

\begin{abstract}
en forme de crochets s'étirant vers l'arrière. Gravure de style dégénéré ; le diamètre du flan inférieur à celui du coin.
\end{abstract}

\section{Bronze au taureau cornupète, série 18 (période III, 140-49 av. J.-C.)}

\section{Les monnaies de la Narbonnaise}

Série au Bouquetin ou Cervidé attribuée traditionnellement aux Cavares ${ }^{13}$, classe III

A/ Tête d'Apollon lauré, à droite.

R/ Taureau chargeant à droite, M A $\Sigma \Sigma$ A au-dessus du taureau Réf. Lyon cat. 206-213 p. 37 ; LT 2242

6- Serrières-de-Briord « Point Vert » (01) ; Bronze frappé ; 15 mm ; 2,15 g.

Bronze au taureau cornupète, émission tardive (imitation?)

A/ Tête d'Apollon lauré, à droite, peu soigné

$\mathrm{R} /$ Fruste

7- St-Baudille-de-la-Tour « Médille »; Bronze frappé12 $; 13 \mathrm{~mm} ; 1,66 \mathrm{~g}$.

A/ Tête laurée à droite, gravée avec peu de soin.

$\mathrm{R} /$ Bouquetin courant à droite, la queue au-dessus du dos ; au-dessous, une croix.

A. Deroc (Deroc 1983) date cette série du premier quart du Ier s. av. J.-C. Elle pourrait être plus ancienne.

Réf. Lyon cat. 143-145 p. 63 ; Deroc cl. III. 


\section{Série en argent au cheval galopant attribuée traditionnellement aux Cavares ${ }^{14}$, classe II}

8- Villebois (01) ; Argent frappé ; 14 mm ; 2,28 g.

A/ Tête laurée à gauche; grènetis au pourtour ; chevelure et bandeau stylisés en trois rangées parallèles de motif : traits obliques, chevrons, virgules.

$\mathrm{R} /$ Cheval galopant à gauche ; la crinière est représentée par un grènetis ; au-dessus du cheval, un rameau ou une épée, symbolisé par une ligne bouletée à son extrémité et accostée de quatre points ; cercle plein au pourtour.

A. Deroc place cette frappe vers $90-75$ av. J.-C., mais cette datation est considérée comme trop tardive.

Réf. Lyon cat. 151-15 p. 64 ; Renne cat. 1497-1501 p. 140 ; LT 2895 ; BN 2637-44/2893-95, Deroc cl. II.

9- Villebois (01); Argent frappé ; $13 \mathrm{~mm} ; 2,15 \mathrm{~g}$.

10-Villebois (01) ; Argent frappé ; $15 \mathrm{~mm} ; 2,23 \mathrm{~g}$.

11- Villebois (01) ; Argent frappé ; $13 \mathrm{~mm} ; 2,18 \mathrm{~g}$.

12- Villebois (01) ; Argent frappé ; $14 \mathrm{~mm} ; 2,21 \mathrm{~g}$.

13- Villebois (01) ; Argent frappé ; $14 \mathrm{~mm} ; 2,21 \mathrm{~g}$.

Série en argent au cheval galopant attribuée traditionnellement aux Cavares, classe III

A/ Tête laurée à gauche, très schématisée; grènetis.

$\mathrm{R} /$ Cheval galopant à gauche ; la crinière est représentée par un grènetis ; au-dessus du cheval, un rameau ou une épée, symbolisé par une ligne bouletée à son extrémité et accostée de quatre points; au-dessous, VOL.

Réf. Lyon cat. 159-162 p. 64 ; BN 2628-2635; Deroc cl. III.

14- Porcieu-Amblagnieu (38); Argent fourré, frappé ; $15 \mathrm{~mm} ; 147$ g. ; VOL, revers fruste.

\section{Obole des Volques Arécomiques (?) imitant l'obole de Marseille du groupe IV}

A/ Tête nue d'Apollon à gauche, sans corne frontale; les mèches encadrant le front et les tempes sont en forme de crochets s'étirant vers l'arrière. Gravure de style dégénéré

$\mathrm{R} /$ Roue à quatre rayons dont la jante a disparue ; A R entre les rayons.

Cette monnaie peut être rapprochée chronologiquement du monnayage massaliote qu'elle imite, soit vers 90-49 av. J.-C. Réf. RIG IV, ${ }^{\circ} 35$ p. 90-91 ${ }^{15}$.

15- Optevoz « Batailler est »; monnaie n 107 ; Argent frappé ; $10 \mathrm{~mm} ; 0,42 \mathrm{~g}$.

\section{Série à l'Hippocampe des Allobroges, classe I}

A/ Tête casquée à droite ; le casque à cimier avec panache est stylisé ; grènetis. Le cou est parfois orné d'un torque. Sur certains exemplaire le profile est en buste et cuirassé (monnaie $\left.\mathrm{n}^{\circ} 13\right)$.

R/ Hippocampe stylisé à droite ; cercle de grènetis. 


\section{Le monnayage au cavalier de la vallée du Rhône} prototype les exemplaires de Q MARCIUS PHILIPUS monétaire vers 119 av. J.-C. Les émissions, attribuées aux Allobroges, sont placées entre 75 et 52/51 av. J.-C., mais cette datation tardive est controversée.

\section{Série au cavalier des Allobroges, en argent, anépigraphe} g. ; []/CO[MA] 

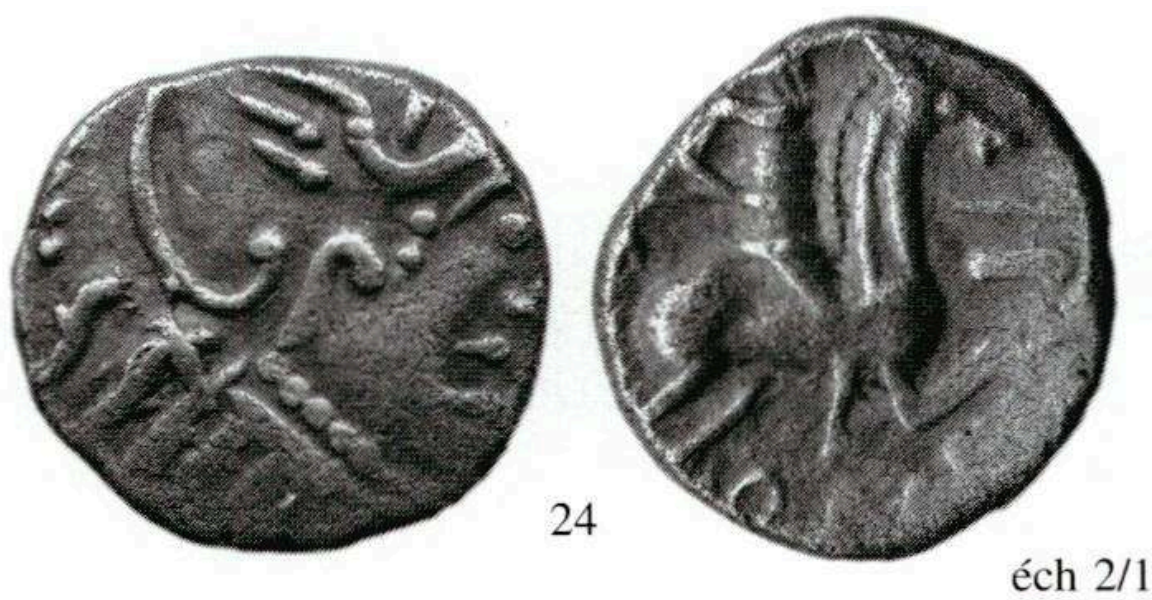

Série au cavalier des Allobroges, en argent, groupe I, 7 ; avec rameau et annelet

114 A/ Tête casquée de Rome à droite ; devant un rameau et BR-I ; grènetis.

$115 \mathrm{R} /$ Cavalier armé d'une lance au galop à droite; au-dessous COMA surmonté d'un annelet centré.

116 Réf. Lyon cat. $198-210$ p. 65 ; Renne cat. 1512 p. 141 ; LT 5836 ; Deroc groupe 1, 7 ; BN 5833-5854 ; Colbert 1973, p. 296-298

117 25- Faverges, commune de Creys-Mépieu-Pusignieu ; Argent frappé ; $15 \mathrm{~mm} ; 1,61 \mathrm{~g}$. (monnaie en partie coupée) ; []/C[OMA]

Série au cavalier des Allobroges, en argent, groupe II, 16

A/ Tête casquée de Rome à droite ; devant ROW ; grènetis.

R/ Cavalier armé d'une lance au galop à droite ; au-dessous CN.VOL ; grènetis.

Réf. Lyon cat. 246-248 p. 66-67 ; Renne cat. 1516 p. 142 ; LT 5895 ; Deroc groupe 11,16 ; BN 5895-5905 ; Colbert 1973, p. 296-298

26- St-Vulbas (01) ; Argent frappé ; 17 mm ; 2,12 g. ; [RO]W/CN.VOL

Série au cavalier des Allobroges, en argent, groupe III, 18

A/Tête casquée de Rome à droite ; derrière AMBILLI ; grènetis.

R/ Cavalier armé d'une lance au galop à droite ; au-dessous EBVRO ; grènetis.

Réf. Lyon cat. 253-256 p. 67 ; Deroc groupe 111,18 ; LT 5719 ; Colbert 1973, p. 296-298 

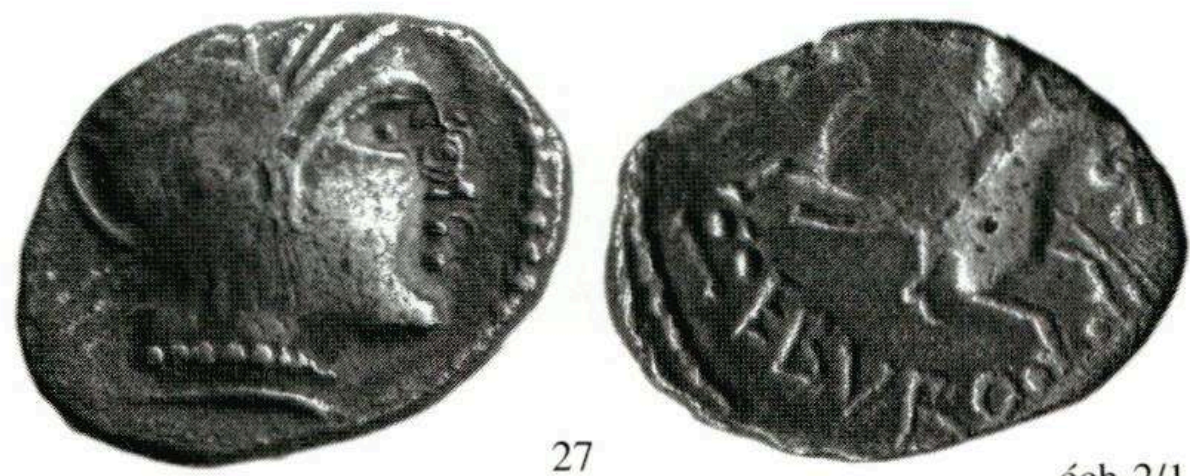

éch $2 / 1$

\section{[AMBIL]LI/EBVRO}

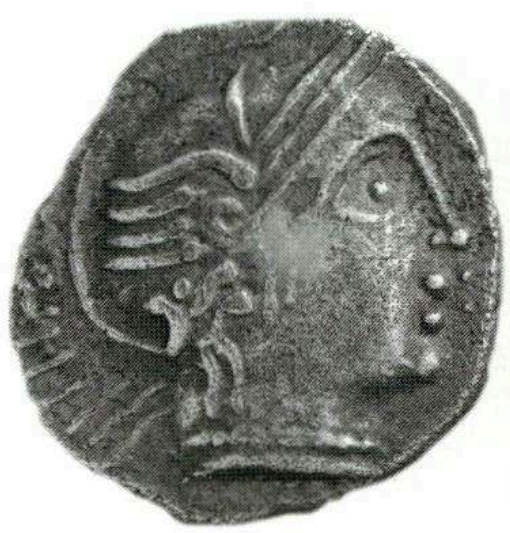

28

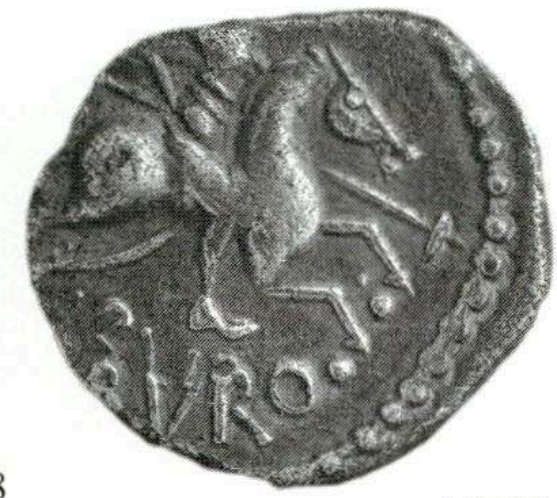

éch $2 / 1$

28- Faverges, commune de Creys-Mépieu-Pusignieu ; Argent frappé ; $15 \mathrm{~mm} ; 2,05$ g. ; [AMJBILLL/[E]BVRO ${ }^{16}$

29- Faverges, commune de Creys-Mépieu-Pusignieu ; Argent frappé ; 15 mm ; 1,95 g. ; légende non lisible

30- La Brosse, commune de La-Balme-Les-Grottes ; Argent fourré, frappé ; $15 \mathrm{~mm} ; 1,11$ g. ; []/EBVR[O] ; avers fruste.

Série au cavalier des Allobroges, en argent, groupe IV, 23

A/ Tête casquée de Rome à droite ; devant DVRNACOS ; grènetis.

R/ Cavalier armé d'une lance au galop à droite ; au-dessous AVSCRO ; grènetis.

Réf. Lyon cat. 263-273 p. 67-68 ; Renne cat. 1519-1524 p. 142 ; LT 5762 ; Deroc groupe IV, 23 ; BN 5749-5771; Colbert 1973, p. 296-298

31- St-Vulbas (01) ; Argent frappé ; 15 mm ; 1,77 g. ; [D]VRNACO[S]/AVSCRO

32- St-Vulbas (01) ; Argent frappé ; $14 \mathrm{~mm} ; 1,79 \mathrm{~g}$. ; légende non lisible

33- St-Vulbas (01) ; Argent frappé ; $15 \mathrm{~mm} ; 1,63 \mathrm{~g}$. ; DVRNACOS/[] 

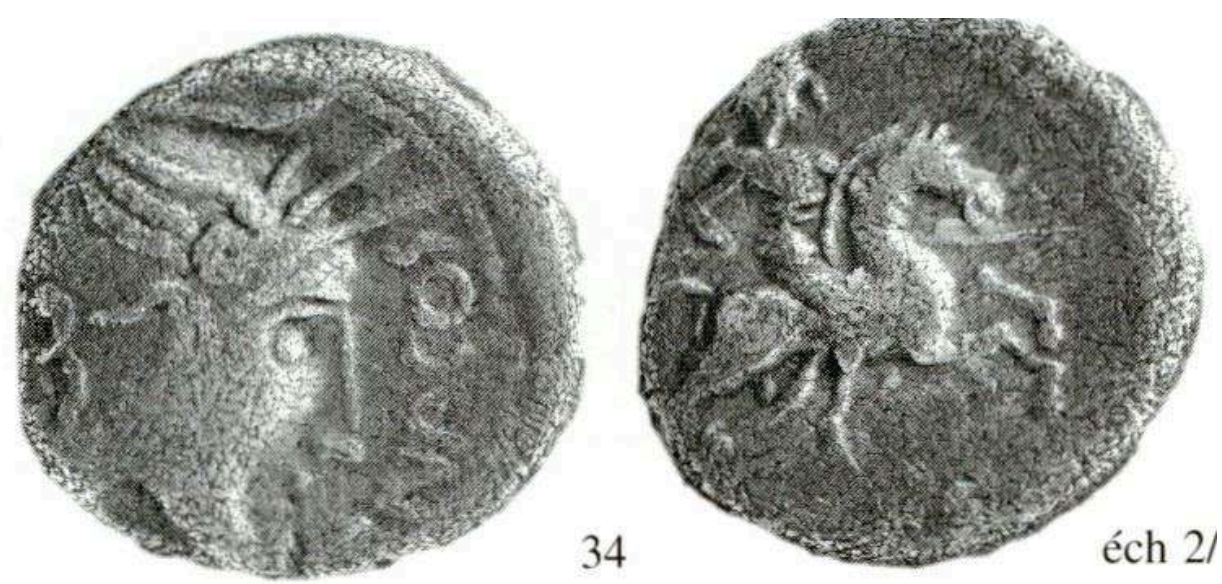

\section{Rhône DVRNACC
contemporaine,}

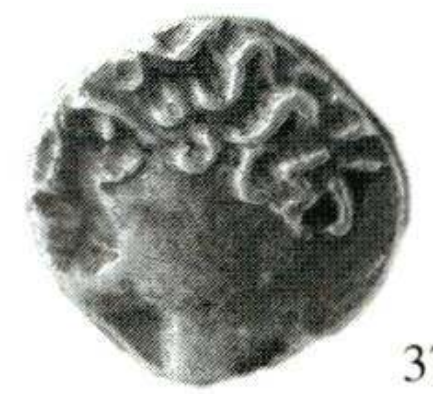

37

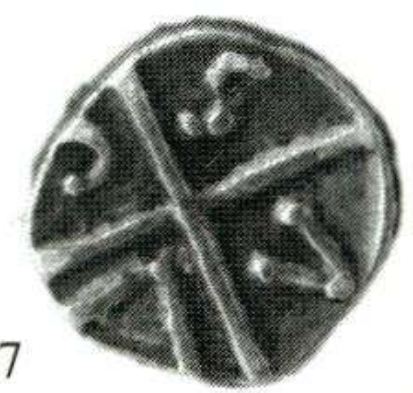

éch 2/1

\section{Série des statères imitant le monnayage de Philippe II de Macédoine, type d'Annonay}


$144 \mathrm{R} /$ Char à deux chevaux, vers la gauche, conduit par un aurige. Il semble s'agir d'une émission tardive de statères, car en or bas ou fourré. Le style du profil au droit de ces monnaies, rappelle les monnayages au bouquetin et au cheval attribués traditionnellement aux Cavares. La datation de ces monnaies, qui appartiennent probablement aux numéraires de la vallée du Rhône (Van der Wielen 1999 :72, note 101), doit se situer dans la première moitié du Ier s. av. J.-C. Une origine allobroge paraît envisageable si l'on accepte, pour le même peuple, la provenance des monnayages au bouquetin et au cheval galopant.

Réf. Lyon cat. 314 p. $73-74$; Deroc p. 24 ; LT 6067 ; BN 6066-6067.

38- Faverges commune de Crey-Mépieu-Pusignieu ; Or bas fourré ; $18 \mathrm{~mm} ; 5,04 \mathrm{~g}$.

39- Villebois (01); Or bas ; $19 \mathrm{~mm} ; 6,32 \mathrm{~g}$.

\section{Les monnaies de la Gaule Chevelue}

Les monnaies du centre et du centre-est de la Gaule

Série des potins à la grosse tête du centre-est, attribués aux Séquanes.

Potin à la grosse tête au bandeau lisse, type A4,1

A/ Tête humaine grossière à gauche; un double bandeau lisse limite la chevelure ; la calotte crânienne remplit tout l'espace entre le bandeau et le pourtour du flan, divisant ainsi l'image monétaire en deux; l'oeil est indiqué par une cavité ; le nez est séparé de la face; petit front; la lèvre supérieure est indiquée par un petit trait, la lèvre inférieure par un globule important ; le cou figuré par deux barres au relief aplati,

$\mathrm{R} /$ Quadrupède à corne, vers la gauche; une longue queue fortement recourbée audessus du dos; la patte avant formant un angle ouvert et touchant presque en son extrémité la ligne de sol créée par la patte arrière repliée à angle droit; filet périphérique. Ces monnaies sont attachées, dans de nombreux cas, à des contextes de La Tène D1. Ce type de monnayage est antérieur à 52 av. J.-C.

Réf. Geiser et Gruel 1993 type A4, 1 ; Lyon cat. 537-544 p. 91 ; Renne cat. 1283 p. 119 ; LT 5368 et 5401 ; Geiser et Gruel 1996 p. 11-15.

40- St-Vulbas (01) ; Bronze coulé ; $19 \mathrm{~mm} ; 5,46 \mathrm{~g}$.

Potin à la grosse tête à bandeau décoré, type B, 1

A/ Grosse tête à gauche; oeil en creux cerclé par un anneau; oeil et nez reliés; joue prononcée; globule en guise de lèvre inférieure; double bandeau décoré : perles ou feuilles ; un petit cou figuré par deux barres en relief aplati ; filet périphérique.

$\mathrm{R} /$ Quadrupède à corne, tourné vers la gauche, dérivé du taureau cornupète de Marseille; tête courte et fine, œil globulaire; échine redressée, la queue fortement recourbée au-dessus du dos touche presque la corne ;

Réf. Geiser et Gruel 1993 type B1 ; Geiser et Gruel 1996 p. 11-15.

41- St-Baudille-de-la-Tour « La Plaine »; monnaie n 93 ; Bronze coulé ; $19 \mathrm{~mm} ; 6,23 \mathrm{~g}$. 
Potin à la grosse tête à bandeau mixte, type B, 5

157 A/ Grosse tête à gauche; petit oeil réaliste; bouche représentée par deux petits globules ; oreille figurée par deux cercles jointifs ; double bandeau mixte représenté par une bande lisse et une bande perlée ; légende $Q$ DOCI ; filet périphérique

$\mathrm{R} /$ Quadrupède à corne, tourné vers la gauche; tête haute; queue en forme de $\mathrm{S}$ audessus du dos qui touche la corne dans certain cas.

Ce monnayage en bronze coulé, de même que son parallèle à la légende Q SAM, font partie d'un groupe comprenant les quinaires à la légende Q DOCI SAM F. L'émission de ces quinaires est antérieure à $52 \mathrm{av}$. J.-C. et ne peut remonter au-delà de 57 av. J.-C., les potins sont probablement contemporains de cette frappe.

Réf. Geiser et Gruel 1993 type B5 ; Geiser et Gruel 1996 p. 11-15 ; LT 5542 ; BN 5542-5545 ; Lyon cat. 531-532 p. 91 ; Renne cat. 1290-1291 p. 120.

42- St-Baudille-de-la-Tour « La Plaine »; monnaie n 92 ; Bronze coulé ; 18 mm ; 4,40 g. ; DOCI
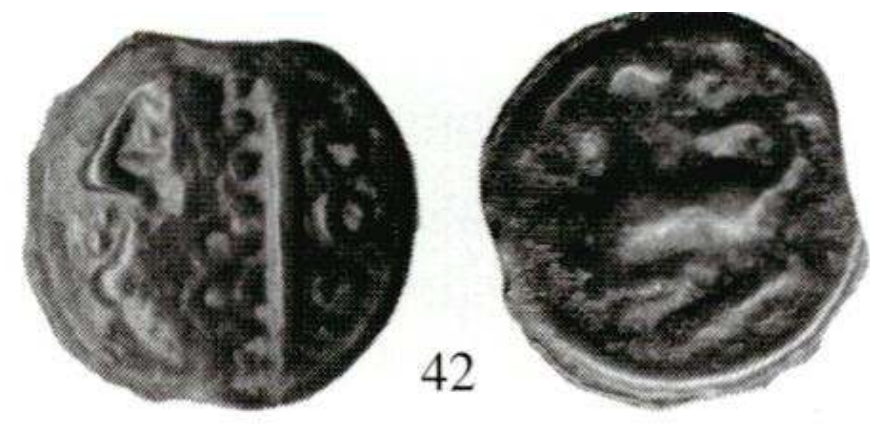

\section{$100 \%$}

\section{Potin au swastika de la Loire moyenne}

A/ Tête réduite à une silhouette et apparemment casquée, à droite ; filet périphérique

$\mathrm{R} /$ Swastika dont les bras se touchent ; filet périphérique.

La forte similitude qui existe entre le droit de ces monnaies et ceux des potins à la tête diabolique des Turones, en fait une série parallèle et sans doute contemporaine, dont l'émission, largement représentée dans la seconde moitié du Ier s. av. J.-C., ne semble pas remonter au-delà de la guerre des Gaules.

5 Réf. Lyon cat. 822-828 p. 116 ; Fischer 1983 p. 291-298 ; BN 6293-6294.

43- Optevoz « Le Couloup »; monnaie n 134 ; Bronze coulé ; 15 mm ; 2,71 g. 

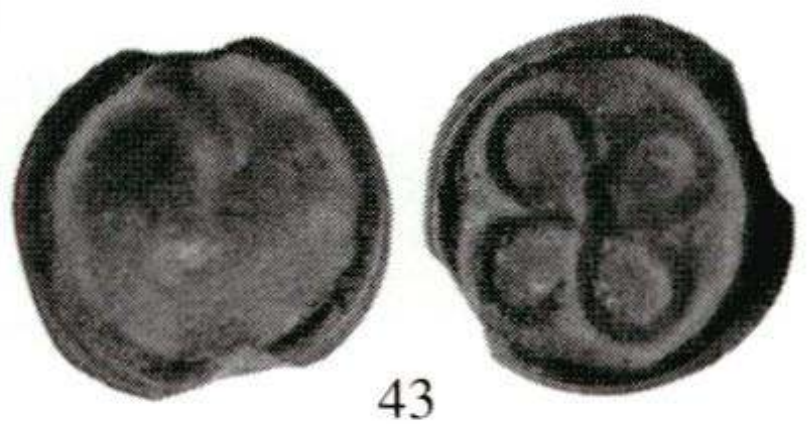

\section{$100 \%$}

Série des quinaires incertains de l'Est, à la légende SEQVANOIOTVOS Lingons, mais une origine éduenne d'une partie de ce type de monnayage n'est pas exclue. Le prototype est un denier romain au nom de P. Cornellius Sulla, émis vers 145 av. J.-C. Les émissions semblent commencées dès la fin du IIe s. av. J.-C., et se poursuivent jusqu'à la conquête romaine.

A/ Tête imberbe à gauche, chevelure représentée par deux rangées de boucles fermées ; grènetis

R/ Sanglier à gauche, les soies hérissées au-dessus du dos; autour se développe la légende SEQVANOIOTVOS

Ces monnaies se rattachent au groupe des quinaires de l'Est, notamment ceux à la légende KA ETE_OY, les plus anciens. Le début de la frappe doit se situer bien avant la guerre des Gaules (seize exemplaires ont été trouvés dans les fossés de Grésigny-SainteReine) ; l'attribution traditionnelle aux Séquanes est incertaine.

Réf. Lyon cat. 569-571 p 93 ; LT 5351 ; BN 5329-5366

44- St-Vulbas (01) ; Argent frappé ; 13 mm ; 1,78 g. ; SEQVANO[IOT-VOS]

\section{Quinaire Lingons à la légende KALETEDV (KAAETEAOY)}

A/ Tête coiffée d'un casque ailé, à gauche ; le cou ceint d'un collier perlé ; grènetis

$\mathrm{R} /$ Cheval bridé galopant vers la gauche; légende (KA^ETE $\triangle O Y$ ) sous la forme d'un monogramme qui évolue selon les émissions, ici : KA $-\mathrm{O} / \mathrm{E}-\mathrm{Y} / \mathrm{E}-\mathrm{Y}$

D'abord classées comme incertaine de l'Est ces monnaies ont été attribuées aux

Réf. Lyon cat. 464-470 p. 87-88; Renne cat. 1367-1371, cl. H, p. 125 ; RIG IV, n 92 p. 169-170.

6 45- St-Baudille-de-la-Tour «Le Cruy »; monnaie $\mathrm{n}^{\circ} 1$; Argent frappé ; $12 \mathrm{~mm}$; ? g; $\mathrm{KA} \Lambda$ - O []

46- Hières-sur-Amby ; Argent fourré, frappé ; 13 mm ; 1,74 g. ; KA - O []

47- Verna ; Argent frappé ; $11 \mathrm{~mm} ; 1,61 \mathrm{~g} .{ }^{18} ; \mathrm{KA} \Lambda$-O[]Y 


\section{Quinaire Eduens EDVIS/ORGETIRIX}
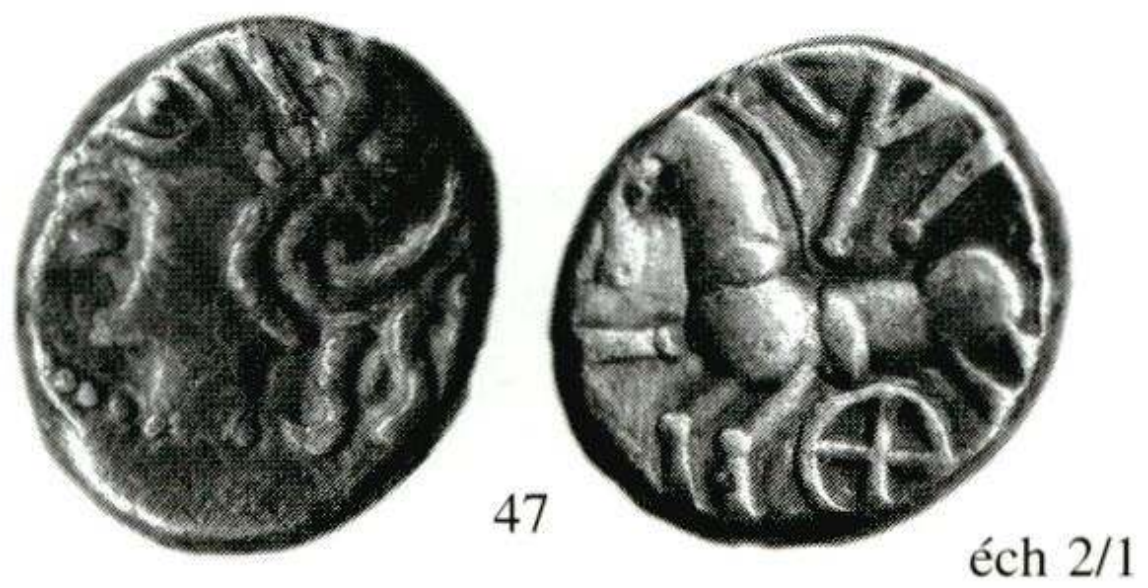

A/ Buste drapé à droite, dont la chevelure est composé de six mèches enroulées; devant EDVIS ; grènetis

R/ Ours avançant vers la droite ; à l'exergue ORGETIRIX ; grènetis Cette série reprend le même profil que «les quinaires à la lyre ", elle pourrait donc être contemporaine. Cependant leur absence des trésors monétaires datés semble indiquer leur postériorité à la guerre des Gaules. La présence de ce type de monnaies dans le trésor de Chantenay (Nièvre) indique une frappe antérieure à 36 av. J.-C.

Réf. Lyon cat. 350-351 p 77-78 ; BN 4822-4829

48- St-Baudille-de-la-Tour « Lacliou »; monnaie $\mathrm{n}^{\circ} 52$; Argent fourré, frappé ; $13 \mathrm{~mm}$; $1,41 \mathrm{~g}$. ; percée d'un trou central ; ED[VIS]/[]
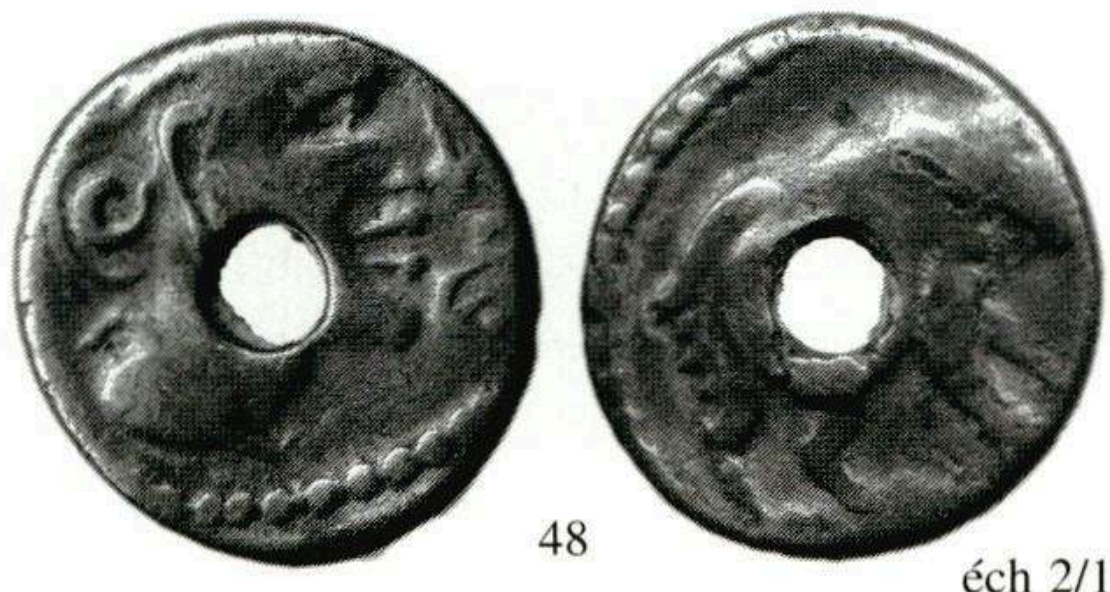

Flan en argent $\left(n^{\circ} 51\right)$

Un élément particulier, pourrait s'avérer être un flan de monnaie gauloise, ou un numéraire à part entière fonctionnant comme un moyen de paiement et/ou d'échange. Il s'agit d'un nodule d'argent, ovale, de $9 \mathrm{~mm}$ de longueur par $6 \mathrm{~mm}$ de largeur, et d'un poids de 1,72 g. $^{19}$. Celui-ci provient d'un petit habitat où les matériaux sont 
caractéristiques d'un établissement gallo-romain, et dont le mobilier permet d'envisager une occupation entre le deuxième quart du Ier s.ap. J.-C. et le troisième quart du IVe s. ap. J.-C. Mais, quelques éléments isolés attestent d'une occupation antérieure, au Ier s. av. J.-C., avec notamment la présence de tessons d'amphores Dressel $1 \mathrm{~A}$ et d'une monnaie gauloise ( $c f$. monnaie $n^{\circ} 47$ ). L'existence de ce type de flan est attestée par la découverte de moules à alvéoles ${ }^{20}$ sur plusieurs oppida, et par la présence de flan de même forme notamment sur l'oppidum de Zàvist (Bohême).

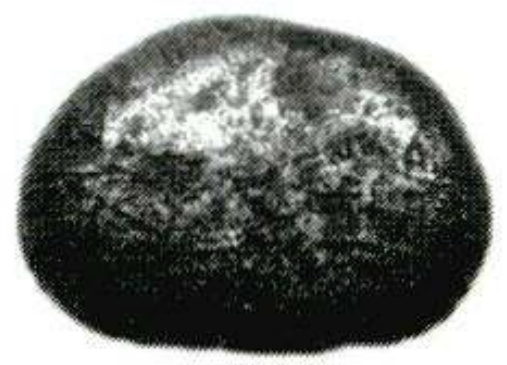

\section{NOTES}

1. Le site, au nord-est de la tombe à char, en est distant d'environ deux kilomètres. Il se manifeste principalement par la présence de tegulae, de céramique commune et de sigillée.

2. Neuf monnaies proviennent de Faverges, commune de Creys-Mépieu-Pusignieu; une de Boulieu commune de Courtenay ; dix de StBaudille-de-la-Tour ; quatre d'Optevoz; une de Hièressur-Amby ; trois de Verna, une de Porcieu-Amblagnieu, et une de La Brosse, commune de LaBalme-Les-Grottes. Ces monnaies, hors stratigraphie, sont issues principalement d'établissements isolés, dont le matériel est caractéristique de la période gallo-romaine (seuls quelques éléments isolés: amphores italiques, céramique campanienne, objets métalliques, monnaies, attestent d'une occupation de La Tène D), et de l'agglomération secondaire, antique, d'Optevoz. Seule la monnaie provenant de Hières-sur-Amby est une découverte isolée, hors contexte, dans un sentier menant du village à Larina.

3. Une monnaie provient de Serrières-de-Briord (le Point Vert); neuf de Villebois; neuf de StVulbas; une de Marcilleux, commune de St-Vulbas. Ces monnaies sont issues du même type d'établissements que précédemment.

4. Ce premier lot rassemble les types les plus anciens, sa composition permet d'envisager un enfouissement plus précoce.

5. La situation de cette région par rapport à un axe de communication entre la Gaule Narbonnaise, la Gaule Chevelue, et l'Italie, ainsi que la présence d'un passage à gué sur le Rhône, au niveau de St-Vulbas, ne sont sans doute pas étranger à ce phénomène. 
6. Ces chiffres sont à prendre avec prudence, le lot étudié n'étant pas très important. Si l'on associe ce lot à celui de Larina, les espèces massaliotes restent bien représentées, largement devant le monnayage originaire de Gaule Chevelue.

7. L'association des deux lots monétaire représente quatre-vingt-dix-sept individus identifiés, dont $23,7 \%$ sont des espèces massaliotes et $12,4 \%$ des espèces provenant de Gaule Celtique.

8. Il est entendu qu'une distribution secondaire peu être à l'origine de cette répartition du lot monétaire.

9. Rappelons qu'un coin monétaire, du revers de ce type de numéraire, avait été découvert en 1987 sur le site de Larina.

10. Brenot et Scheers 1996 ; De La Tour et Fischer 1994.

11. Pour toutes les monnaies concernant les communes de Saint-Baudille-de-la-Tour et d'Optevoz voir Carrara 2000, Vol. II.

12. Le flan de cette monnaie, par sa forme, a sans doute été coulé.

13. Depuis déjà quelques années une origine allobroge est pressentie pour ces monnaies. À la lumière des nouvelles découvertes et des travaux récents, il apparaît que ce type de monnayage soit bel et bien Allobroge (Van der Wielen 1999, Dhénin 2002)

14. Là encore, les nouvelles données disponibles et les travaux effectués ses dernières années, tendent à prouver l'origine allobroge du monnayage au cheval libre galopant. D'ailleurs les deux coins monétaires que l'on connaisse, pour ce type de numéraire, ont été découverts en territoire allobroge, et notamment un à Larina.

15. Colbert de Beaulieu et Fischer 1998.

16. Cette monnaie a la particularité d'avoir été sur-frappée ou mal frappée, provoquant ainsi un dédoublement de la légende EBVRO.

17. A Lyon, les fouilles entreprises sur "le sanctuaire de Cybèle" (sondage D2) ont permis de découvrir cinq exemplaires de ce type de monnayage, dans un contexte stratigraphique de 30/40 av. J.-C., voir 50/60 av. J.-C. (renseignements A. Desbat, A. Audra).

18. Le faible poids des exemplaires $n^{\circ} 46$ et 47 pourrait indiquer qu'il s'agit ici d'exemplaires tardifs ou d'imitations.

19. Le poids de cet élément ( 1,72 g.) est proche des standards de certaines espèces régionales, notamment les cavaliers du Rhône du type DVRNACOS/AVSCRO (1,75 g. en moyenne).

20. Des moules de ce type étaient présents parmi le mobilier de la faille de "La Chuire" à Larina. Voir également Debord 1989. 


\section{Bibliographie}

\section{Abréviations}

B.S.RF. : Bulletin de la Société Préhistorique Française

CN. : Cahier Numismatique

RAC. : Revue archéologique du Centre de la France (Tours).

RAE. : Revue archéologique de l'Est et du Centre-Est (Dijon).

RAN. : Revue archéologique de Narbonnaise (Montpellier).

RAO. : Revue archéologique de l'Ouest (Rennes).

\section{BIBLIOGRAPHIE}

Adam 1995 : ADAM (A.-M.). - À propos de quelques récipients du service funéraire étrusque (VIe-IVe s. av. J.-C.). Revue des Etudes Anciennes 97, 1-2, 1995, p. 103-113.

Adler 1993 : ADLER (W.). - Studien zur germanischen Bewaffnung. Waffenmitgabe und Kampfesweise im Niederelbegebiet und im übrigen Freien Germanien um Christi Geburt. Saarbrücker Beitrage zur Altertumskunde 58. Bonn, 1993.

Alföldi, Radnóti 1937-40 : ALFÖLDI (A.), RADNOTI (A.). - Zügelringe und Zierbeschläge von romischen Jochen und Kummeten aus Pannonien. In : Serta Hoffilleriana. Vjestnik Hrvatskoga Arh. Drustva N. S. 18-21, 1937-1940, p. 309-319.

Alfoldi et al. 1957 : ALFÖLDI (M.) et al. - Intercisa II. (Dunapentele). Geschichte der Stadt in der Romerzeit ; Archaeologica Hungarica 36 ; Budapest, 1957.

Amedick 1993 : AMEDICK (R.). - Zur Ikonographie der Sarkophage mit Darstellungen aus der Vita Privata und dem Curriculum Vitae eines Kindes. In : G. KOCH (dir.), Grabeskunst der romischen Kaiserzeit. Mainz, 1993, 143-153. 
Amy et al. 1962 : AMY (R.), DUVAL (P.-M.), FORMIGE (J.), HATT (J.-J.), PIGANIOL (A.), PICARD (CH.), PICARD (G.-CH.). - L'arc d'Orange. Paris, 1962 (Suppl. à Gallia ; 15).

Ankner, Hummel 1985 : ANKNER (D.), HUMMEL (F). - Kupferlote bzw. Verkupferung auf Eisen. Arbeitsbldtter für Restauratoren, Heft 2, 1985, Gruppe 1 (Eisen), p. 196-206.

Arcelin 1980 : ARCELIN (P.). - Nouvelles observations sur la nécropole préromaine de La Catalane aux Baux-deProvence (B.-du-Rh.). Bulletin de l'École Antique de Nîmes, 15, 1980, p. 91-110.

Arcelin 1981 : ARCELIN (P.). - Les céramiques de type celtique en Provence. R. A. E., 32, fasc. 3-4, p. 33-66.

Artaud 1846 : ARTAUD (F.). - Lyon souterrain, ou observations archéologiques et géologiques faites dans cette ville depuis 1874 jusqu'à 1836. Lyon : Montfalcon, 1846.

Babeş 1993 : BABEș (M.).-Die Poienessti-LukasevkaKultur. Ein Beitrag zur Kulturgeschichte im Raum ostlich der Karpaten in den letzten Jahrhunderten vor Christi Geburt. Saarbrücker Beitrage zur Altertumskunde 3. Bonn, 1993.

Baranowski 1973 : BARANOWSKI, (T.). - Rzasd konskiz wodzami lanncuchowymi na terenie europy srodkowej w okresie wplywow rzymskiu. Archeologia Polski 18, 1973, p. 391-477.

Baratte 1989 : BARATTE (F.). - Trois vases en argent d'époque républicaine trouvés dans la Saône à Thorey (commune de Saint-Germain-du-Plain, Saône-et-Loire). R. A. E., n 40, 1989, p. 61-74.

Baratte et al. 1984 : BARATTE (Fr.), BONNAMOUR (L.), GUILLAUMET (J.-P), TASSINARI (S.). - Vases antiques de métal au Musée de Chalon-sur-Saône. Dijon, 1984 (Suppl. à la R. A. E. ; 5).

Barrai, Colin, Luginbühl 1998 : BARRAL (P.), COLIN (A), LUGINBUHL (T.). - Les importations méditerranéennes : la vaisselle céramique. In : GRUEL (K.), VITALI (D.) dir. - L'oppidum de Bibracte : un bilan de onze années de recherche (1984-1995). Gallia 55, 1998, 1-140. p. 73-75.

Barruol, Sauzade 1969 : BARRUOL (G.), SAUZADE (G.). - Une tombe de guerrier à Saint-Laurent-des-Arbres (Gard). Contribution à l'étude des sépultures du 1er s. av. J.-C. dans la basse vallée du Rhône. Rivista di Studi Liguri 35,1969, p. 15-89.

Bataille 2001 : BATAILLE (G.). - Les agrafes de ceinturon du sanctuaire de La Villeneuve-au-Châtelot (Aube). Archäologisches Korrespondenzblatt 31, 2001, p. 443-460.

Bats 1990 : BATS (M.). - Tombes et nécropoles de Narbonnaise aux IIe-Ier s. av. J.-C. : problèmes de datation et de chronologie. In : DUVAL (A.), MOREL (J.-P), ROMAN (Y.) dir. - Gaule interne et Gaule méditerranéen aux IIe et Ier siècles avant J.-C. : confrontations chronologiques, actes de la table-ronde de Valbonne (1986). Paris : CNRS, 1990. (Suppl. à la R. A. N. ; 21).

Baumgàrtel 1937 : BAUMGÄRTEL (E.). - The Gaulish Necropolis of Filottrano in the Ancona Museum. Journal of the Royal Anthropological Institute, LXVII, 1937, p. 231-286.

Behrens 1923 : BEHRENS (G.). - Denkmaler des Wangionengebietes. Frankfurt : Germanische Denkmaler der Frühzeit 1, 1923.

Behrens 1927 : BEHRENS (G.). - Bodenurkunden aus Rheinhessen. Bilderheft zur Vor-und Frühgeschichte Rheinhessens. I. Die vorrömische Zeit. Mainz, 1927.

Berger et al. à paraître : BERGER (J.-F.), ROYET (R.), ARGANT (J.) et FOREST (V.). - Une villa gallo-romaine en milieu humide. Le Vernai à Saint-Romain-de-Jalionas (Isère) In : Actes du colloque AGER, à paraître.

Bernigaud 2002 : BERNIGAUD (N.). - Contribution à l'étude des anthroposystèmes des marais de l'ouest de l'isle Crémieu (Isère). Mémoire de DEA, Université Paris I, 2002.

Blanequaert 1998 : BLANCQUAERT (G.). - Cottévrard « La Plaine de la Bucaille » (Seine Maritime). Présentation préliminaire de la nécropole laténienne. Revue Archéologique de Picardie 1/2, 1998, p. 171-183.

Bockius 1990 BOCKIUS (R.). - Eingefriedete endlatènezeitliche Gräber bei Thür, Kr. Mayen-Koblenz. In : WEGNER (H.-H.) éd., Berichte zur Archäologie an Mittelrhein und Mosel 2, Trierer Zeitschrift Beiheft 12. Trier, 1990, p. 145-168. 
Bockius 1991 : BOCKIUS (R.). - Zu den elbgermanischen Kreisplattensporen der frühen römischen Kaiserzeit. Jahrbuch RGZM 38, 1991, p. 497-514.

Bocquet 1967-70 : BOCQUET (A.). - Catalogue des collections préhistoriques et protohistoriques. Grenoble : Musée Dauphinois, 1967-1970.

Bocquet 1969 : BOCQUET (A.). - L’Isère pré - et protohistorique. Gallia Préhistoire XII, 1969, fasc. 1, p. 121-258, 63 pl. et fasc. 2, p. 273-400, 119 pl.

Bocquet 1976 : BOCQUET (A.). - La tombe de Verna (Isère) et les mégalithiques alpins. Etudes Préhistoriques 13, 1976, p. 23-32, 10 fig.

Bocquet 1991 : BOCQUET (A.). - Nouvelles tombes gauloises en Dauphiné. In : DUVAL (A.) dir. - Les Alpes à l'âge du Fer. Paris, 1991, p. 247-253 (Suppl. à la R. A. N. ; 22).

Bocquet, Haussmann 2001 BOCQUET (A.) et HAUSSMANN (L.). - Dernières découvertes protohistoriques en Nord-Dauphiné et en Savoie. B.S.P.F.. n 98, 2001, p. 299-310

Boessneck et al. 1971 : BOESSNECK (J.), VON DEN DRIESCH (A.), MEYER-LEMPENAU (U.), WECHSLERVON OHLEN (E.). - Die Tierknochenfunde aus dem Oppidum von Manching. Die Ausgrabungen in Manching 6. Wiesbaden, 1971.

Bonnantour 1989 : BONNAMOUR (L.). - Nouvelles découvertes archéologiques en Saône sur le site du gué de Gigny/Thorey (Saône-et-Loire). R. A. E., n 40, 1989, p. 47-60.

Bonuci, Graziani 1975 : BONUCI (E.), GRAZIANI (G.) - Comparative thermogravimetric, X-ray diffraction and electron microscope. Investigations of burnt bones from recent, ancient and prehistoric age, Academia Nazionale dei Lincei, serie 8, vol. 59, fasc. 5, 1975, p. 518-533.

Bordenache Battaglia 1979 : BORDENACHE BATTAGLIA (G.). -Le ciste prenestine, I : Corpus 1. Rome, 1979.

Bottini 1993 : BOTTINI (A.). dir. -Armi. Gli strumenti della guerra in Lucania. Le mostre, i cataloghi, vol. 2. Bari, 1993.

Boucher 1986 : BOUCHER (St.). - Problèmes concernant une anse étrusque. In : Italian Iron Age Artefacts in the British Museum. Papers of the sixth British Museum Classical Colloquium. Londres, 1986, p. 107-115.

Boucher, Tassinari 1976 : BOUCHER (St.), TASSINARI (S.). - Bronzes antiques du Musée de la civilisation galloromaine à Lyon. I. inscriptions, statuaire, vaisselle. Lyon, 1976.

Boulestin, Buisson, Gomez de Soto 2002 : BOULESTIN (B.), BUISSON (J.-F.), GOMEZ DE SOTO (J.). - Tombes sans défunt, d'époque laténienne et du Haut-Empire : pour une relecture hérétique. In : GUICHARD (V.), PERRIN (F.) dir. : - L'aristocratie celte à la fin de l'âge du Fer (IIe s. av. J.C., Ier s. ap. J. C.). Actes de la table ronde des 10 et II juin 1999 (Glux-en-Glenne). Glux-en-Glenne : Centre archéologique du Mont Beuvray, 2002, p. 189-197 (Bibracte ; 5).

Bourgogne médiévale 1987 : Bourgogne médiévale, la mémoire du sol. 20 ans de recherches archéologiques. Paris, 1987.

Božič 1984 : BOZIC (D.). - O starosti konjeniškega groba št. 16 latenskega grobišča na beograjski Karaburmi. In : GUSTIN (M.), PAULI (L.), éd.. Keltske Voz. Posavski Muz. Brežice 6 : Brezice, 1984, p. 133-139.

Brenot, Scheers 1994 : BRENOT (C.), SCHEERS (S.). - Catalogue des monnaies massaliètes et des monnaies celtiques du musée des Beaux-Arts de Lyon, Leuven. In : De La TOUR (H.), FISCHER (B.), Atlas des monnaies gauloises. Paris (mise à jour de l'ouvrage paru en 1892), 1994.

Bretz-Mahler 1959 : BRETZ-MAHLER (D.). - Les torques ornithomorphes de la Tène I. B.S.P.F., $\mathrm{n}^{\circ}$ 56, 1959, p. 493-499.

Brizio 1899 : BRIZIO (E.). - Il sepolcreto gallico di Montefortino presso Arcevia. Monumenti Antichi 9, 1899, p. 616-792.

Brunaux 1999 : BRUNAUX (J.-L.), dir. - Ribemont-sur-Ancre (Somme). Bilan préliminaire et nouvelles hypothèses. Gallia 56, 1999, p. 177-283. 
Brunaux, Rapin 1988 : BRUNAUX (J.-L.), RAPIN (A.). - Gournay II. Boucliers et lances. Dépôts et trophées. Revue Archéologique de Picardie. Paris, 1988.

Brunaux et al. 1985 : BRUNAUX (J.-L.), GOGUEY (R.), GUILLAUMET (J.-P.), MÉNIEL (P.), RAPIN (A.). - Le sanctuaire celtique de Mirebeau (Côte-d'Or). In : Les Ages du Fer dans la vallée de la Saône (VIIe-Ier 5. avant notre ère). Paléométallurgie du bronze à l'âge du Fer. Actes du Septième colloque de l'AFEAF, Rully 1983. Paris, 1985, p. 79-111. (Suppl. à la R. A. E. ; 6).

Bruyère 1986 : BRUYERE (G.). - Essai de bibliographie descriptive de François Artaud (1767-1838), archéologue. Position de mémoire de maîtrise. In : Travaux de l'Institut d'Histoire de l'Art de Lyon, cahiers 10, 1986, p. 60-77, fig. 25 et 28 .

Buchsenschutz et al. 1988 : BUCHSENSCHUTZ (O.) et al. - L'évolution du canton de Levroux d'après les prospections et les sondages archéologiques. Levroux I., 1988 (Suppl. à la R. A. C. ; 1).

Cahen-Delhaye 1993 : CAHEN-DELHAYE (A.). - Reconstitution d'un char à deux roues de La Tène d'après les données de fouilles en Ardenne Belge. Première partie : analyse des données archéologiques. ArchéoSitula 17-20, 1993, p. 55-70.

Caillaud 2001 : CAILLAUD (C.). - La villa gallo-romaine de Saint-Romain-de-Jalionas (Isère) et son environnement archéologique dans la cité des Allobroges. Mémoire de maîtrise, 2 vol., Université Paris I, 2001.

Carrara 2000 : CARRARA (S.). - L'occupation antique du plateau de L'Isle Crémieu : Les communes de St-Baudillede-la-Tour et d'Optevoz. Mémoire de maîtrise, Université Lyon II, 2000.

Challet 1992 : CHALLET (V.). - Les Celtes et l'émail. Doc. Préhist. 3. Paris, 1992.

Chantre 1880 : CHANTRE (E.). - Etudes paléo-ethnologiques dans le bassin du Rhône, Premier âge du Fer, nécropoles et tumulus. Paris, Lyon : Baudry, 1880.

Chantre 1892 : CHANTRE (E.). — La nécropole gauloise de Gênas (Isère). Bulletin de la Société anthropologique de Lyon, 11, 1892, p. 124-125.

Chantre 1899 : CHANTRE (E.). - Les nécropoles gauloises du bas Dauphiné : Leyrieux, Rives et Genas. Comptes rendus de l'Académie des inscriptions et Belles-Lettres, Lyon, 1899, p.768-771.

Chantre 1900 : CHANTRE (E.). - Nouvel inventaire des monuments mégalithiques dans le Bassin du Rhône. Bulletin de la Société anthropologique de Lyon 19, 1900. p. 24-25.

Chantre 1923 : CHANTRE (E.). - Le Camp retranché de Larina, commune d'Anoisin-Chatelan, près de Crémieux (Isère). Rhodania, Congrès de Vienne, 1923, p. 64-68.

Chapotat 1970 : CHAPOTAT (G.). - Vienne gauloise : le matériel de La Tène III trouvé sur la colline de SainteBlandine. Lyon : Faculté des Lettres et Sciences humaines, 1970, 2 vol. (Publications du CERGR ; fasc. 2).

Colbert de Beaulieu 1965 : COLBERT DE BEAULIEU (J.-B. ). - Les monnaies de Caletedu et le système du denier en Gaule. C.N. 2, p. 163-180.

Colbert de Beaulieu 1966 : COLBERT DE BEAULIEU (J.-B.). - « La monnaie de Caletedu et les zones du statère et du denier en Gaule » RAC. 18, p. 101-129.

Colbert de Beaulieu 1973 : COLBERT DE BEAULIEU (J.-B.). - Traité de numismatique Celtique, I, Méthodologie des ensembles. Paris.

Colbert de Beaulieu, Fischer 1998 : COLBERT DE BEAULIEU (J.-B.), FISCHER (B.). - Recueil des Inscriptions Gauloises, Les Légendes Monétaires IV, 1998 (Suppl. à Gallia ; XLV).

Colin 1998 : COLIN (A.). - Chronologie des oppida de la Gaule non méditerranéenne. Paris : MSH (DAF ; 71).

Cordie-Hackenberg, Haffner 1997 CORDIE-HACKENBERG (R.). HAFFNER (A.). - Das keltischrömische Gräberfeld von Wederath-Belginum. 5. Teil : Gräber 1818-2472, ausgegraben 1978, 1981-1985, mit Nachträgen zu Band 1-4. Trierer Grabungen und Forschungen 6, 5. Mainz, 1997.

Crişan 1980 : CRIşAN (I. H.). - Necropola dacică de la Cugir (jud. Alba). Apulum 18, 1980, p. 81-87.

Crișan 1993 : CRIŞAN (I. H.). - Civilizatśia geto-dacilor, 1993. 
Cristofani 1985 : CRISTOFANI (M.). - I bronzi degli Etruschi. Novara, 1985.

Daicoviciu 1954 : DAICOVICIU (C.). - Cetatea daciâ de la Piatra Rossie. Monographie archeologicâ ; Bucuresti, 1954.

Dainville de 1968 : DAINVILLE de (F.). — Le Dauphiné et ses confins vus par l'ingénieur d'Henry IV, Jean de Beins. Genève : Librairie Droz, 1968.

Déchelette 1913 : DECHELETTE (J.). - La collection Millon. Antiquités préhistoriques et gallo-romaines. Paris, 1913.

Debord 1989 : DEBORD (J.). - L'atelier monétaire gaulois de Villeneuve-St-Germain. RN, 6e série, 31, 1989.

Debord 1996 : DEBORD (J.). - Le faciès monétaire de Villeneuve-Saint-Germain et ses éléments de datation. Gallia 52, 1996, p. 61-78.

Dedet et al. 1978 : DEDET (B.), MICHELOZZI (A.), PY (M.), RAYNAUD (C.), TENDILLE (C.). - Ugernum. Protohistoire de Beaucaire. Cahier de l'Association pour la recherche archéologique en Languedoc Oriental, 6 ; Caveirac, 1978.

Dedet 1992 :DEDET (B.). - Rites funéraires protohistoriques dans les garrigues languedociennes. Paris : CNRS, 1992, 413 p. (Suppl. à la R. A. N. ; 24).

Deroc 1983 : DEROC (A.). - Les monnaies d'argent de la vallée du Rhône. Paris, 1983.

Deroc et Vian 1971 : DEROC (A.), VIAN (P.-C.). - Les monnaies gauloises d'argent de la vallée du Rhône. CN. 8, p. 69-93.

Deyber et al. 1984 : DEYBER (A.), DALAUT (M.), LADIER (E.), WEISROCK (A.) — L'habitat fortifié laténien de « La Pierre d'Appel » à Étival-Clairefontaine (Vosges). Gallia 42, 1984, p. 175-217.

De Marinis 1997 : DE MARINIS (R.). - La tomba gallica di Castiglione delle Stiviere (Mantova). Notizie Arch. Bergomensis 5, 1997, p. 115-177.

Dhénin 2002 : DHENIN (M.). - Le monnayage Allobroge In : Les Allobroges : Gaulois et Romains du Rhône aux Alpes. Catalogue d'exposition du Musée Dauphinois. Grenoble : In folio, 191p.

Dicocer 2 : PY (M.), ADROHER AUROUX (A.M.), SANCHEZ (C.). - Dicocer : corpus des céramiques de l'âge du Fer de Lattes. Lattes : ADALR, 2001.2 vol. (Lattara ; 14).

Dobesch 1996 : DOBESCH (G.). - Überlegungen zum Heerwesen und zur Sozialstruktur der Kelten. In : Die Kelten in den Alpen und an der Donau. Akten des internationalen Symposions St. Pölten 1992. Archaeolingua 1. Budapest, Wien, 1996, p. 13-71.

Dohrn 1982 : DOHRN (T.). - Die etruskische Kunst im Zeitalter der griechischen Plastik. Mainz, 1982.

Dolenz 1998 : DOLENZ (H.). - Das Händlerfuhrwerk aus SH/5 und ausgewählte eiserne Wagenbestandteile. In : PICCOTTINI (G.), éd., Die Ausgrabungen auf dem Magdalensberg 1980 bis 1986. MagdalensbergGrabungsberichte 16 ; Klagenfurt, 1998, p. 529-567.

Duday 1981 : DUDAY (H.). - Etude des restes osseux provenant de la nécropole de Las Peyros à Couffoulens (Aude), groupes 1 et 2, fouilles 1977. R. A. N, n 14, 1981.

Dufourg, Antoine 1978 : DUFOURG (A.), ANTOINE (J. L.). - A propos du passé protohistorique de SaintRomain-de-Jalionas. Evocations, 1978, p. 11-14.

Dufourg 1980 : DUFOURG (A.). - Le site de Saint-Romain de-Jalionas dans son contexte archéologique. Bulletin de liaison de la DAH Rhône-Alpes 9. 1980, p. 10-27.

Dupuis 1940 : DUPUIS (J.). - Une tombe à char gauloise de La Tène I. Revue Archéologique 16, 1940, p. 62-68.

Duval, Verron 1993 : DUVAL (A.), VERRON (G.). - La tombe avec éléments de char de Marcilly-sur-Eure (Eure) La Croix du Breuil. In : Les Celtes en Normandie. Les rites funéraires en Gaule (Ille-Ier s. av. J.-C.). Rennes, 1993, p. 135-147 (Suppl. à la R. A. O. ; 6). 
Egg 1996 : EGG (M.). - Das hallstattzeitliche Fürstengrab von Strettweg bei Judenburg in der Obersteiermark. Mainz : Monographien RGZM 37, 1996.

Egg, France-Lanord 1987 : EGG (M.), FRANCE-LANORD (A.). - Le char de Vix. Mainz : Monographien RGZM $13,1987$.

Egg, Pare 1995 : EGG (M.), PARE (Ch.). - Die Metallzeiten in Europa und im vorderen Orient. Die Abteilung Vorgeschiclite im Römisch-Germanischen Zentralmuseum. Kataloge Vor-und Frühgeschichtlicher Altertümer 26. Mainz, 1995, p. 202, pl. couleur XXV, 1.

Eggers 1951 : EGGERS (H. J.). - Der römische Import im freien Germanien. Atlas der Urgeschichte vol. 1. Hamburg, 1951.

Eggers 1965 : EGGERS (H. J.). - Der Grabfund von Markkleeberg-Gautzsch bei Leipzig. In : Studien aus Alteuropa Teil II. Beiheft Bonner Jahrbücher 10/11 ; Köln, Graz, 1965, p. 201-207.

Engels 1970 ENGELS (H.-J.). - Eine bronzene Schwertscheide der Spatlatènekultur aus Ludwigshafen. Pfälzer Heimat 21, 1970, 81-84.

Engels 1974 : ENGELS (H.-J.). - Funde der Latènekultur I. Materialheft zur Vor - und Frühgeschichte der Pfalz 1 ; Speyer, 1974.

Evans 1890 : EVANS (A. J.). - On a Late-Celtic Urn-Field at Aylesford, Kent, and on the Gaulish, Illyro-Italic, and Classical Connexions of the Forms of Pottery and Bronzework there discovered. Archaeologia 52/2, 1890, p. 315-388.

Fauduet 1992 : FAUDUET (I.). - Vestiges de La Tène finale à Argentomagus. Saint Marcel (Indre). In : Actes du XIIIe colloque de l'AFEAF Le Berry et le Limousin... (1992) p. 239-244.

Ferdière, Villard 1993 : FERDIERE (A.), VILLARD (A.). - La tombe augustéenne de Fléré-la-Rivière (Indre) et les sépultures aristocratiques de la cité des Bituriges. Saint-Marcel, 1993 (Suppl. à la R. A. C. ; 7).

Feugère 1989 : FEUGERE (M.)· - Gobelet tardo-républicain en bronze provenant d'Agde (Hérault).

Archéologie en Languedoc 1989, p. 7-8.

Feugère 1994 : FEUGERE (M.). — Les Fouilles de la Mairie de Besançon (Doubs) (1989-1990). Petits objets des niveaux latèniens. In : JUD (P.), éd., Die spätkeltische Zeit am südlichen Oberrhein. Kolloquium Basel 1991. Basel, 1994. p. 146-155.

Feugère 1996 : FEUGERE (M.). - Un espace funéraire du deuxième quart du Ier s. av. J.-C. Nîmes, Gard. Gallia 52,199, p. 165-204.

Feugère, Rolley 1991 : FEUGERE (M.), ROLLEY (CL), éd. - La vaisselle tardo-républicaine en bronze. Actes de la table-ronde Lattes 1990. Dijon : Université de Bourgogne, Centre des recherches sur les techniques grécoromaines, 13, 1991.

Filow 1937 : FILOW (B.). - Die Kuppelgräber von Mezek. Bull. Inst. Arch. Bulgare 11, 1937, p. 1-116.

Fingerlin 1999 : FINGERLIN (G.). - Römische und keltische Reiter im Lager der XIX. Legion von Dangstetten am Hochrhein. Archäologische Nachrichten aus Baden 60, 1999, p. 3-18.

Fischer 1983 : FISCHER (B.) - Les Potins de type BN 6284-6294 et leurs variantes. CN. 20, p. 291-298, 1983.

Fischer 1991 : FISCHER (B.). - Les monnaies marseillaises et celtiques de Larina, (Hières-sur-Amby, Isère). In : DUVAL (A.) éd. - Les Alpes à l'âge du Fer. Actes du Xe colloque de TAFEAF, Yenne-Chambéry 8-11 mai 1986. Paris : CNRS, 1991, p. 45-55 (Suppl. à la R. A. N. ; 22).

Fischer 1959 : FISCHER (F.). - Der spatlatènezeitliche Depot-Fund von Kappel (Kreis Saulgau). Urkunden zur Vorund Frühgeschichte aus Südwürttemberg-Hohenzollern, vol. 1. Stuttgart, 1959.

Fitzpatrick 1987 : FITZPATRICK (A.). - Die Eimer vom Typus Fällanden : Ein italischer Bronzegefäßtyp des 1. Jh. v. Chr. Jahrbuch der Schweizerischen Gesellschaft für Ur-und Frühgeschichte 70, 1987, 101.112.

Fol 1991 : FOL (A.). -The Chariot-Burial at Mezek. In : The Celts. Milano. 1991. 
Fouet, Savès 1971 : FOUET (G.), SAVES (G.). - Le bronze à Vieille-Toulouse : Trouvailles anciennes. R.A.N., $\mathrm{n}^{\circ}$ 4, 1971, p. 47-92.

Frey 1976 : FREY (O.-H.). - The Chariot Tomb from Adria : Some Notes on Celtic Horsemanship and Chariotry. In : MEGAW (J. V. S.) éd. - To illustrate the monuments. Essays on archaeology presented to Stuart Piggott on the occasion of his sixty-fith birthday. London, 1976, p. 172-179.

Frey 1984 : FREY (O.-H.). - Ein seltenes Zaumzeug aus Caporetto/Kobarid. In : Preistoria del Caput Adriae. Atti Convegno Internazionale Trieste 1983 ; Udine, 1984, p. 119-129.

Frey 1986 : FREY (O.-H.). - Zeugnisse sog. thrakischer Trensen in keltischen Oppida. Nachträge zu einer Verbreitungskarte latènezeitlicher Hebelstangengebisse. In : Beiträge zur Eisenzeit. Kleine Schriften aus dem Vorgeschichtlichen Seminar Marburg vol. 19. Marburg, 1986, p. 37-42.

Froquet 1994 : FROQUET (H.). - Larina, étude typologique du matériel céramique protohistorique. Mémoire de M.S.T ; dirigé par J. Vital. Université Lyon 2.

Furger-Gunti, Berger 1980 : FURGER-GUNTI (A.), BERGER (L.). - Katalog und Tafeln der Funde aus der spätkeltischen Siedlung Basel-Gasfabrik. Baseler Beiträge zur Ur-und Frühgeschichte vol. 7 ; Derendingen, 1980.

Gabut 1894 : GABUT (F.). - Archéologie préhistorique et gallo-romaine : Larina, Marigneu. Tombeau du Général, l'Ile Barbe. In : La Construction lyonnaise. Lyon : impr. A. Rey, 1984, p. 1-52.

Galli e l'Italia 1978. - I Galli e l'Italia. Catalogue de l'exposition, Rome, 1978.

Gauer 1981 : GAUER (W.). - Ein spätarchaischer Beckengriff mit Tierkampfgruppe. In : X. Bericht über die Ausgrabungen in Olympia. Berlin, 1981, p. 111-165.

Gauer 1991 : GAUER (W.). - Die Bronzegefässe von Olympia, I. Berlin : Olympia-Forschungen XX, 1991.

Gebhard 1991 : GEBHARD (R.). - Die Fibeln aus dem Oppidum von Manching. Die Ausgrabungen in Manching 14 ; Stuttgart, 1991.

Gehrig 1981 : GEHRIG (U.). - Le trésor d'Hildesheim. Dossiers Histoire et archéologie 54. juin 1981, p. 22-37.

Gerlach 1976 : GERLACH (G.). - Dus Gräbeifeld. « Die Motte » bei Lebach. Katalog. Saarbrücker Beiträge zur Altertumskunde, vol. 16 ; Bonn, 1976.

Glodariu, Iaroslavschi 1979 : GLODARIU (I.), IAROSLAVSCHI (E.). - Civilizatia fierului la Daci. Bucarest, 1979.

Goudineau 2002 : GOUDINEAU (Chr.) - Dynasties gauloises, dynasties romaines dans les Trois Gaules. In: GUICHARD (V.), PERRIN (F.) dir. - L'aristocratie celte à la fin de l'âge du Fer (IIe s. av. J. -C, Ier s. ; ap. J.-C.). Actes de la table-ronde des 10 et 11 juin 1999 (Glux-en-Glenne - F. 58). Glux-en-Glenne : Centre archéologique européen du Mont Beuvray, 2002, p. 311-317 (Bibracte ; 5).

Gran-Aymerich 1998 : GRAN-AYMERICH (E.). - Naissance de l'Archéologie moderne, 1789-1945. Paris : CNRS Editions, 1998.

Grappin 1985 : GRAPPIN (S.). - Le site du « Verger » aux âges du Fer. Saint Romain (Côte-d'Or). In : Les âges du Fer dans la vallée de la Saône (VIIe-Ier s. avant notre ère). Paléométallurgie du bronze à l'âge du Fer. Actes du septième colloque de l'AFEAF, Rully 1983. Paris, 1985, p. 53-64, (Suppl. à la R. A. E. ; 6).

Graue 1974 : GRAUE (J.). - Die Gräberfelder von Ornavasso. Eine Studie zur Chronologie der späten Latène-und frühen Kaiserzeit. Hamburger Beiträge zur Archäologie Beiheft vol. 1 ; Hamburg, 1974.

Gruel, Geiser 1996 : GRUEL (K.), GEISER (A.). - Les potins à la grosse tête. In : Les Potins Gaulois : Typologie, Diffusion, Chronologie. Gallia 52, 1996, p. 11-20.

Gruel, Vitali 1998 : GRUEL (K.), VITALI (D.), dir. - L'oppidum de Bibracte. Un bilan de onze années de recherche (1984-1995). Gallia 55, 1998, p. 1-140.

Guadagnin 1978 : GUADAGNIN (R.). - La nécropole celtique de Bouqueval. Bulletin de la Jeunesse Préhistorique et Géologique de la France 8, 1978, p. 12-65.

Guichard et al. 1993 : GUICHARD (V.), PION (P.), MALACHER (F.), COLLIS (J.). - À propos de la circulation monétaire en Gaule chevelue aux IIe et Ier s. av. J.-C. R. A. C., n 32, 1993, p. 25-55. 
Guillaumet 1979 : GUILLAUMET (J.-P.). - La défaite des Helvètes - Histoire et archéologie. In : DUVAL (P.M.), KRUTA (V.). - Les mouvement celtiques du Ve au Ier s. avant notre ère. Actes du XXVIIIe colloque organisé à l'occasion du IXe Congrès International des Sciences Préhistoriques et Protohistoriques, Nice 1976. Paris, 1979, p. 195-200.

Guillaumet 1993 : GUILLAUMET (J.-P.). - Les fibules de Bibracte. Techniques et typologie. Dijon : Publications du Centre de Recherches sur les Techniques Gréco-Romaines 14, 1993.

Guillaumet, Szabó 1985 : GUILLAUMET (J.-P.), SZABÓ (M.). - Sur quelques vases en bronze d'importation du musée de Chalon-sur-Saône. In : Les âges du Fer dans la vallée de la Saône. VIIe colloque de l'AFEAF Rully 1983. Paris, 1985, p. 71-76.

Guštin 1981 : GUSTIN (M.). - Keltische Gräber aus Dobova, Slowenien. Archäologisches Korrespondenzblatt 11, 1981, p. 223-229.

Guštin 1984a : GUSTIN (M.). - Prazgodovinski grobovi Z vozovi na ozemlju Jugoslavije. In : GUSTIN (M.), PAULI (L.), éd. - Keltske Voz. Posavski Muz. Brežice 6 ; Brežice, 1984, p. 111-132.

Guštin 1984b : GUSTIN (M.). - Die Kelten in Jugoslawien. Ubersicht über das archäologische Fundgut. Jahrbuch RGZM 31, 1984, p. 305-363.

Guštin 1991 : GUSTIN (M.). - Posočje. Posočje in der jüngeren Eisenzeit. Kat. in Monogr. vol. 27 ; Ljubljana, 1991.

Hachmann 1990 : HACHMANN (R.). - Gundestrup-Studien. Untersuchungen zu den spätkeltischen Grundlagen der frühgermanischen Kunst. Berichte der Römisch-Germanischen Kommission 71, 1990, p. 565-903.

Haffner 1971 : HAFFNER (A.). - Das keltisch-römische Gräberfeld von Wederath-Belginum, 1. Teil : Gräber 1-428. ausgegraben 1954/55. Trierer Grabungen und Forschungen, vol. 6,1; Mainz, 1971.

Haffner 1974a : HAFFNER (A.). - Die spätlatènezeitlichen Treverergräber von Konz-Filzen, Kr. TrierSaarburg. Archäologisches Korrespondenzblatt 4, 1974, p. 53-58.

Haffner 1974b : HAFFNER (A.). - Das keltisch-römische Gräberfeld von Wederath-Belginum, 2. Teil : Gräber 429-883, ausgegraben 1956/1957. Trierer Grabungen und Forschungen, vol. 6, 2 ; Mainz, 1974.

Haffner 1978 : HAFFNER (A.). - Das keltisch-römische Gräberfeld von Wederath-Belginum, 3. Teil : Gräber 885-1260, ausgegraben 1958-1960, 1971 u. 1974. Trierer Grabungen und Forschungen, vol. 6, 3 ; Mainz, 1978.

Haffner 1989a : HAFFNER (A.), dir. - Gräber - Spiegel des Lebens. Zum Totenbrauchtum der Kelten und Römer am Beispiel des Treverer-Gräberfeldes Wederath-Belginum. Schriftenreihe Rheinischen Landesmuseums Trier vol. 2 ; Mainz, 1989.

Haffner 1989b : HAFFNER (A.). - Grab 296. Zur pars pro toto-Sitte und rituellen Zerstörung von Waffen während der Latènezeit. In : HAFFNER, (A.), dir. - Gräber - Spiegel des Lebens. Zum Totenbrauchtum der Kelten und Römer am Beispiel des Treverer-Gràberfeldes Wederath-Belginum. Schriftenreihe des Rheinischen Landesmuseums Trier, vol. 2 ; Mainz, 1989, p. 197-210.

Haffner 1989c : HAFFNER (A.). - Das spätlatènezeitliche Kriegergrab 1178 mit Feinwaage. In : HAFFNER, (A.), dir. - Gräber - Spiegel des Lebens. Zum Totenbrauchtum der Kelten und Römer am Beispiel des TrevererGräberfeldes WederathBelginum. Schriftenreihe des Rheinischen Landesmuseums Trier, vol. 2 ; Mainz, 1989, p. 173-186.

Haffner 1992 : HAFFNER (A.). - Die keltischen Fürstengräber des Mittelrheingebietes. In : Hundert Meisterwerke keltischer Kunst. Schmuck und Kunsthandwerk zwischen Rhein und Mosel. Schriftenreihe des Rheinischen Landesmuseums Trier, vol. 7 ; Trier, 1992, p. 31-61.

Haffner, Joachim 1984 : HAFFNER (A.), JOACHIM (H.-E.). - Die keltischen Wagengräber der Mittelrheingruppe. In : GUSTIN (M.), PAULI (L.), éd., Keltske Voz. Posavski Muz. Brezice 6 ; Brežice, 1984, p. 71-87.

Harnecker 1997 : HARNECKER (J.). - Katalog der Eisenfunde von Haltern aus den Grabungen der Jahre 19491994. Bodenaltertümer Westfalens, vol. 35 ; Mainz, 1997. 
Hayen 1983 : HAYEN (H.). - Handwerklich-technische Lösungen im vor-und frühgeschichtlichen Wagenbau. In : JANKUHN (H.) et al. (dir.) - Das Handwerk in vor - und frühgeschichtlicher Zeit. Teil II., Abhandlungen der Akademie der Wissenschaften Göttingen, Philologische-Historische Klasse, 3 Folge, $\mathrm{n}^{\circ}$ 123. Göttingen, 1983, p. 415-470.

Haynes 1985 : HAYNES (S.). - Etruscan Bronzes. London, 1985.

Hecht 1998 : HECHT (Y.). - Die Ausgrabungen auf dem Basler Milnsterhügel an der Rittergasse 4, 1982/6 : Spätlatènezeit und augusteische Epoche. Materialhefte zur Archäologie in Basel 16 ; Basel, 1998.

Henning 1912 : HENNING (R.). - Denkmäler der Elsässischen Altertumssammlung zu Straßburg, 1912.

Heym 1959/61 : HEYM (W.). - Drei Spätlatènegräberfelder aus Westpreußen. Offa, 17/18, 1059/61, p. 143-170.

Horn, Rüger 1979 : HORN (H. G.), RÜGER (Ch. B.), dir. - Die Numidier. Reiter und Könige nördlich der Sahara. Führer des Rheinischen Landesmuseums Bonn vol. 96 ; Köln, 1979.

Hostetter 1986 : HOSTETTER (E.). - Bronzes from Spina. I : The Figurai Classes. Mainz, 1986.

Hübener 1973 : HÜBENER (W.). - Die römischen Metallfunde von Augsburg-Oberhausen. Ein Katalog. Materialhefte zur Bayerischen Vorgeschichte vol. 28 ; Kallmünz, 1973.

Hubert 1902 : HUBERT (H.). - La collection Moreau au Musée de Saint-Germain. Revue Archéologique 41, 1902, p. 167-206.

Jacobi 1974 : JACOBI (G.). - Werkzeug und Gerat aus dem Oppidum von Manching. Die Ausgrabungen in Manching vol. 5 ; Wiesbaden, 1974.

Jahn 1913 : JAHN (M.). - Über Beziehungen zwischen Ostgermanen, Westgermanen und Kelten während der Spät-Latènezeit. Mannus 5, 1913, p. 76-95.

Jamka 1947 : JAMKA (R.). - Grób Z okresu rzymskiegoz Malkowic, pow. piczowski. Wiatowit 18, 1947, p. 179-192.

Jannot 1995 : JANNOT (J.-R.). - Peut-on parler de commerce étrusque en Gaule du Nord-Ouest ? (Façade Manche-Atlantique et arrière-pays, du 8e s. au 2e s. av. J.-C.). In : SWADDLING (J.), WALKER (S.), ROBERTS (P.) (éd.) - Italy in Europe : Economic relations 700BC-AD 50. London : British Museum Occasional Paper, 97, 1995, p. 75-91.

Joachim 1969 JOACHIM (H.-E.). - Unbekannte Wagengräber der Mittel-und Spätlatènezeit aus dem Rheinland. In : FREY (O.-H.), éd. - Marburger Beiträge zur Archäologie der Kelten. Festschrift für Wolfgang Dehn zum 60 Geburtstag am 6 Juli 1969. Bonn : Fundberichte in Hessen, Beiheft 1, 1969, p. 84-111.

Joachim 1973 : JOACHIM (H.-E.). - Ein reich ausgestattetes Wagengrab der Spätlatènezeit aus Neuwied, Stadtteil Heimbach-Weis. Bonner Jahrbuch 173, 1973, p. 1-44.

Joachim 1974 : JOACHIM (H.-E.). - Ein spätlatènezeitliches Reitergrab aus Kollig, Kreis Mayen-Koblenz. Hamburger Beiträge zur Archäologie 4, 1974, p. 159-170.

Joffroy, Bretz-Mahler 1959 : JOFFROY (R.), BRETZ-MAHLER (D.). - Les tombes à char de La Tène dans l'Est de la France. Gallia 17, 1959, p. 5-36.

Jorda, Provansal, Royet 1990 : JORDA (M.), PROVANSAL (M.), ROYET (R.). - L'histoire naturelle d'un site de l'âge du Fer sur le piémont méridional des Alpilles. Le domaine de Servanne (Bouches-du-Rhône). Gallia 47, 1990, p. 57-67.

Jurgeit 1986 : JURGEIT (F.). - Le ciste prenestine, II : Studi e contributi. 1. «Cistenfüsse », Etruskische und Praenestiner Bronzewerkstätten. Rome, 1986.

Kaenel 1983 : KAENEL (G.). - Cinq tombes du second âge du Fer à Sion. Archäologie Schweiz 6. 1983. p. 46-56.

Kaenel, Müller, Furger-Gunti 1984 : KAENEL (G.), MÜLLER (F.), FURGER-GUNTI (A.). - L'occupation celtique du Mont Terri (Jura) sur la base d'anciennes collections jurassiennes. Jahrbuch der Schweizerischen Gesellschaft fur Ur-und Frühgeschichte 67, 1984, p. 95-122. 
Keiling 1984 : KEILING (H.). - Wiebendorf. Ein Urnenfriedhof der frührömischen Kaiserzeit im Kreis Hagenow. Beiträge zur Ur-und Frtihgeschichte der Bezirke Rostock, Schwerin und Neubrandenburg 17 ; Berlin, 1984.

Kessler 1929/30 : KE $\beta$ LER (Р. T.). - Eine neuartige Grabanlage der Latène-Zeit in Wallertheim, Rheinhessen. Mainzer Zeitschrift 24/25, 1929/30, p. 125-133.

Kimmig 1990 : KIMMIG (W.). - Zu einem etruskischen Beckengriff aus Borsdorf in Oberhessen. Archöologisches Korrespondenzblatt 20, 1990, p. 75-85.

Knez 1977 : KNEZ (T.). - Keltski grobovi iz Roj pri Moravcah. In : Keltiske Studije. Posavski Muz Brežice 4 ; Brežice, 1977, p. 105-125.

Kossinna 1905 : KOSSINNA (G.). - Verzierte Eisenlanzenspitzen als Kennzeichen der Ostgermanen. Zeitschrift fur Ethnologie 37, 1905, p. 369-407.

Kotera-Feyer 1993 : KOTERA-FEYER (E.). - Die Strigilis. Frankfurt : Europäische Hochschulschriften Reihe 38 Archäologie Band 43, 1993.

Kramer 1964 KRÄMER (W.). - Latènezeitliche Trensenanhänger in Omegaform. Germania 42,1964, p. 250-257.

Krämer 1985 : KRÄMER (W.). - Die Grabfunde von Manching und die latènezeitlichen Flachgräber in Südbayern. Stuttgart : Die Ausgrabungen in Manching 9, 1985.

Krier, Reinert 1993 : KRIER (J.), REINERT (F.). - Das Reitergrab von Hellingen. Die Treverer und das römische Militär in der frühen Kaiserzeit. Luxemburg, 1993.

Kull 1996 : KULL (B.). - Ein kaiserzeitliches Zaumzeug mit Zügelketten aus Vizsoly, Komitat Borsod-AbaújZemplén (Ungarn). Germania 74, 1996, p. 415-432.

Künzl 1988 : KÜNZL (E.). - Der römische Triumph. Siegesfeiern im antiken Rom. München, 1988.

Künzl 1993 : KÜNZL éd., (E.). - Die Alamannenbeute aus dem Rhein bei Neupotz Plünderungsgut aus dem römischen Gallien. Monographien RGZM vol. 34, 1 ; Mainz, 1993.

Lambot 1989 : LAMBOT (B). - Le sanctuaire gaulois et gallo-romain da Nanteuil-sur-Aisne, lieu-dit Nepellier (Ardennes). Bulletin de la Société Archéologique Champenoise 82/4, 1989, p. 33-44.

Lambot 1998 : LAMBOT (B.). - Les morts d'Acy-Romance (Ardennes) à La Tène finale. Pratiques funéraires, aspects religieux et hiérarchie sociale. In : LEMAN-DELERIVE (G.), dir. - Les Celtes : rites funéraires en Gaule du Nord entre le VIe et le Ier siècle avant Jésus-Christ. Recherches récentes en Wallonie. Namur : Études et Documents, série Fouilles 4, 1998, p.75-87.

Lambot 2002 : LAMBOT (B.). - Noblesse, aristocratie et signes extérieurs de richesse à La Tène Finale en Champagne, In : GUICHARD (V.), PERRIN (F.) dir. -L'aristocratie celte à la fin de l'Age du Fer (IIe s. av. J.C., Ier s. ap. J.-C.). Actes de la table ronde des 10 et 11 juin 1999 (Glux-en-Glenne). Glux-en-Glenne : Centre archéologique du Mont Beuvray, 2002, p. 87-108 (Bibracte ; 5).

Lambot, Friboulet, Méniel 1994 : LAMBOT (B), FRIBOULET (M.), MENIEL (P.). - Le site protohistorique d'AcyRomance (Ardennes). II. Les nécropoles dans leur contexte régional (Thugny-Trugny et tombes aristocratiques) 1986-1988-1989. Reims : Mémoire de la Société Archéologique Champenoise 8, 1994 (Suppl. au Bulletin 2).

Landolfi 1987 : LANDOLFI (M.). - Presenze galliche nel Piceno a sud del fiume Esino. In :Celti ed Etruschi nell'Italia centro-settentrionale dal $V$ secolo a.C. alla romanizzazione. Atti del Colloquio Internazionale (Bologna 12-14 aprile 1985). Imola, 1987, p. 443-468.

Lavendhomme, Guichard 1997 : LAVENDHOMME (M.-O.), GUICHARD (V.). - Rodumna (Roanne, Loire), le village gaulois. Paris : MSH, 1997 (DAF ; 62).

Lejars 1996 : LEJARS (T.). - L'armement des celtes en Gaule du nord à la fin de l'époque gauloise. Revue Archéologique de Picardie, 1996, 3/4, p. 79-103.

Lequoy 1993 : LEQUOY (M.-C.). - Le dépôt funéraire de la Mailleraye-sur-Seine. In : Les celtes en Normandie. Les rites funéraires en Gaule (IIIe s. - Ies. av. J.C.). Actes du 14ème colloque de l'AFEAF, Evreux - mai 1990. Rennes, 1993, p. 121-133 (Suppl. à la R. A. O., 6). 
Lindenthal, Rupp 2000 : LINDENTHAL (J.), RUPP (V.). - Reiche Villengräber in der Wetterau. In : Die Romer zwischeti Alpen und Nordmeer. Zivilisatorisches Erbe einer europäischen Militärmacht. München : Schriftenreihe der Archaologischen Staatssammlung 1, 2000. p. 171-175.

Lucas 2002 : LUCAS (G.). - Les Allobroges dans les sources littéraires. In : Les Allobroges, Gaulois et Romains du Rhône aux Alpes. De l'Indépendance à la période romaine (4e siècle av.J.-C. - 2e siècle apr. J.-C.). Infolio éd., 2002, p. 26-29.

Luczkiewicz 1998 : LUCZKIEWICZ (P.). - Zu ausgewählten balkanischen Waffen der spätlatènezeitlichen Przeworsk-Kultur in Polen. Archäologisches Korrespondenzblatt 28,1998, p. 253-267.

Luik 2000 : LUIK (M.). - Die Funde aus den römischen Lagern um Numantia im Romisch-Germanischen Zentralmuseum. Kataloge vor-und frühgeschichtlicher Altertumer 31; Mainz, 2002.

Maier 1985 MAIER (R. A.). - Ein römischer Brandopferplatz bei Schwangau und andere Zeugnisse einheimischer Religion in der Provinz Ratien. In : Forschungen zur provinzialrömischen Archäologie in BayerischSchwaben. Augsburg : Schwäbische Geschichtsquellen und Forschungen 14, 1985, p. 231-256.

Mandy, Monin, Krausz 1990 : MANDY (B.), MONIN (M.), KRAUSZ (S.). - L'hôpital Sainte-Croix à Lyon : un quatrième fossé. Gallia 47, 1990, p. 79-102.

Mansel 2000 : MANSEL (K.). - Spätrepublikanische Bronzegeschirr aus der Siedlung vom Morro de Mezquitilla (Algarrobo, Malaga). Madrider Mitteilungen 41, 2000, p. 197225.

Marcadal 2000 : MARCADAL (Y.). - Les nécropoles de Mouriès. In : CHAUSSERIE-LAPREE (J.), dir. - Le temps des Gaulois en Provence. Martigues, 2000, p. 244-247.

Mariën 1961 : MARIËN (M.-E.). - La période de la Tène en Belgique. Le Groupe de la Haine. Bruxelles : Monographies d'archéologie nationale 2, 1961.

Mariën 1994 : MARIËN (M.-E.). - Quatre tombes romaines du IIIe s. Thorembais-Saint-Trond et Overhespen. Bruxelles, 1994.

Maule 1977 : MAULE (Q.). - A Near-classical Sculptural Style in Italy. American Journal of Archaeology 81, 1977, p. 487-505.

Mediolanum 1988 : Mediolanum, une bourgades gallo-romaine. 20 ans de recherches archéologiques. Dijon : Exposition, Musée archéologique de Dijon, 1988.

Méniel 1987 : MENIEL (P.). - Chasse et élevage chez les Gaulois (450-52 av. J.-C.). Paris, 1987.

Menke 1991 : MENKE (M.). - Borsdorf, Filottrano, Waldalgesheim. Germania 69, 1991, p. 389-399.

Metzler 1994 : METZLER (J.). - Treverische Reitergräber von Goeblingen-Nospelt. In : Trier. Augustusstadt der Treverer. Stadt und Land in vor-und frtihrömischer Zeit (Exposition Trier). Mainz, 1994, p. 87-99.

Metzler 1995 : METZLER (J.). - Das treverische Oppidum auf dem Titelberg (G.-H. Luxemburg). Zur Kontinuität zwischen der spätkeltischen und der frühromischen Zeit in Nord-Gallien. Luxembourg : Dossiers d'Archéologie du Musée National d'Histoire et d'Art 3, 1995.

Metzler et al. 1991 : METZLER (J.), WARINGO (R.), BIS (R.), METZLER-ZENS (N.). - Clemency et les tombes de l'aristocratie en Gaule Belgique. Luxembourg : Dossiers d'Archéologie du Musée National d'Histoire et d'Art 1, 1991.

Metzler-Zens, Metzler 1998 : METZLER-ZENS (N.), METZLER (J.). - Die spätkeltische Aristokratie in Gallien. Überlegungen zur Selbstdarstellung einer sozialen Grappe. In : Studien zur Archäologie der Kelten, Römer und Germanen in Mittel-und Westeuropa. Alfred Hajfner zum 60. Geburtstag gewidmet. Internationale Archaologie Studia Honoria 4 ; Rahden/Westf., 1998, p. 417-427.

Metzler-Zens et al. 1999 : METZLER-ZENS (N.), METZLER (J.), MÉNIEL (P.), BIS (R.), GAENG (C.), VILLEMEUR (I.). - Lamadelaine, une nécropole de l'Oppidum du Titelberg. Luxembourg : Dossiers d'Archéologie du Musée National d'Histoire et d'Art 6, 1999.

Milne 1944 : MILNE (M. J.). - A Greek Footbath in the Metropolitan Museum of Art. American Journal of Archaeology 48, 1944, p. 26-63. 
Miniero 1987 : MINIERO (P.). - Studio di un carro romano dalla villa c. d. di Arianna a Stabia. Mélanges de l'Ecole Française à Rome, Antiquité 99, Rome, 1987, p. 171-209.

Miron 1986/1987 : MIRON (A.). - Katalog der mittel-und spatlatènezeitlichen Grabfunde im Saarland. Teil 1 : Die Kreise Merzig-Wadern und Saarlouis. Berichte der Staatlichen Denkmalpflege Saarland 27/28, 1986/87, p. 103-43.

Molin 1984 : MOLIN (M.). - Quelques considérations sur le chariot des vendanges de Langres (HauteMarne). Gallia 42, 1984, p. 97-114.

Morel 1990a : MOREL (J.-P.). - Les échanges entre la Grande Grèce et la Gaule du VIIe au Ier s. av. J.-C. In : Atti del XXIX Convegno di Studi sulla Magna Grecia (Tarante 1989). Naples, 1990, p. 247-293.

Morel 1990b : MOREL (J.-P.). - La céramique à vernis noir. In : PERRIN (F.). - Un dépôt d'objets gaulois à Larina, Hières-sur-Amby, Isère. Lyon : Circonscription des Antiquités Historique Rhône-Alpes, 1990, p. 113-116, (DARA ; 4).

Morel 1997 : MOREL (J.-P.). - Céramique à vernis noir. In : LAVENDHOMME (M.-O.), GUICHARD (V.) dir. Rodumna (Roanne, Loire) : le village gaulois. Paris : MSH, 1997, p. 119-128 (DAF ; 62).

Mostra dell'Etruria 1960 : Mostra dell'Etruria padana e della città di Spina. Catalogue de l'exposition, Bologna, 1960.

Muir 2000 : MUIR (S.). - Le cheval. Sellerie \& Équipement. Paris : éditions Solar, 2000.

Müller 1913 : MÜLLER (H.). - Tombes gauloises de La Tène II, découvertes au pied des Balmes de Voreppe (Isère). Bulletin de la Société Dauphinoise d'Ethnologie et d'Archéologie, 20/2-3, 1913, p. 105-113.

Müller 1990 : MÜLLER (F.). - Der Massenfund von der Tiefenau bei Bern. Zur Deutung latènezeitlicher Sammelfunde mit Waffen. Basel : Antiqua 20, 1990.

Müller 1997 : MÜLLER (M.). - Die römischen Bronzegefäße von Haltern. Ausgrabungen und Funde in WestfalenLippe 9/A ; 1997, p. 5-40.

Müller-Karpe 1951 : MÜLLER-KARPE (H.). - Zeugnisse der Taurisker in Kärnten. Carinthia I, 141, 1951, p. 593-677.

Müller-Karpe, Millier-Karpe 1977 : MÜLLER-KARPE (A.), MÜLLER-KARPE (M.). - Neue latènezeitliche Funde aus dem Heidetränk-Oppidum im Taunus. Germania 55, 1977, p. 33-63.

Nahrgang 1967 : NAHRGANG (K.). - Die Bodenfunde der Ur-und Frühgeschichte im Stadt-und Landkreis Offenbach am Main. Frankfurt, 1967.

Nantes 1999 : Nos ancêtres les Gaulois aux marges de l'Armorique. Exposition Musée Dobrée. Nantes, 1999.

Neth 2000 : NETH (A.). - Zum Fortgang der Ausgrabungen in der zweiten Viereckschanze bei Nordheim, Kreis Heilbronn. Archäologische Ausgrabungen in Baden-Württemberg, 1999 (2000), p. 75-79.

Neugebauer 1992 : NEUGEBAUER (J.-W.), dir. - Die Kelten im Osten Österreichs. Wissenschaftliche Schriftenreihe Niederösterreich 92/93/93 ; St. Pölten, Wien, 1992.

Nothdurfter 1979 : NOTHDURFTER (J.). - Die Eisenfunde von Sanzeno im Nonsberg. Mainz : RömischGermanische Forschungen 38, 1979.

Normandie 1990 : Les Celtes en Normandie. Exposition Evreux 1990 ; Paris, 1990.

Nuber 1972 : NUBER (U.). — Ein römischer Grabfund aus Ludwigshafen-Maudach. Mitteilungen des Historischen Vereins der Pfalz 70, 1972, p. 111-137.

Oesterwind 1989 : OESTERWIND (B.-C.). - Die Spätlatènezeit und die frühe Römische Kaiserzeit im Neuwieder Becken. Bonn : Bonner Hefte zur Vorgeschichte 24, 1989.

Oesterwind 1996 : OESTERWIND (B.-C.). - Der Niedergang der keltischen Macht. Kriegswesen und Bewaffnung im Spiegel der mittelrheinischen Grabfunde des letzten vorchristlichen Jahrhunderts. In : SCHÄFER (K.), éd., Caesar am Mittelrhein. Begleitheft zur Sonderausstellung im Stadtmuseum Andernach. Andernacher Beiträge 12 ; Andernach, 1996, p. 83-100. 
Pare 1992 : PARE (Ch. F. E.). - Wagons and Wagon-Graves of the Early Iron Age in Central Europe. Oxford : Oxford University Commitee for Archaelogy Monograph 35, 1992.

Pauli 1993 : PAULI (J.). - Die latènezeitliche Besiedlung des Kelheimer Beckens. Materialhefte zur Bayerischen Vorgeschichte A 62 ; Kallmünz. 1993.

Pautreau 1991 : PAUTREAU (J.-P.). - Sépulture aristocratique augustéenne à Antran (Vienne), note préliminaire. Archäologisches Korrespondenzblatt 21, 1991, p. 271-282.

Pautreau 1999 : PAUTREAU (J.-P), dir. -Antran (Vienne). Un ensemble aristocratique du premier siècle. Poitiers, 1999.

Peacock 1977 : PEACOCK (D.S.P.). - Recent discoveries of Roman kilns in Italy. Antiquaries Journal 57, 1977, p. 262-269.

Pélatan 1986 : PELATAN (J.-P). - L’oppidum de Larina (Isère). Revue archéologique Sites, hors série ; 31, 1986, $102 \mathrm{p}$.

Pelletier 1982 : PELLETIER (A.), Vienne antique : De la conquête romaine aux invasions alamanniques (IIe av.-IIIe ap. J.-C.). Roanne : Horwarth.

Pernice 1925 : PERNICE (E.). - Gefässe und Geräte aus Bronze. Die Hellenistische Kunst in Pompeji IV. Berlin, Leipzig, 1925.

Perrin 1990 : PERRIN (F.). - Un dépôt d'objets gaulois à Larina, Hières-sur-Amby, Isère. Lyon : Circonscription des Antiquités Historique Rhône-Alpes, 1990 (DARA ; 4).

Perrin, Bellon 1992 : PERRIN (F.), BELLON (C.). - Mobilier d'origine et de filiation méditerranéennes dans la moyenne vallée du Rhône entre Alpes et Massif Central. In : Marseille grecque et la Gaule. Aix-en-Provence : A.D.A.M. éd., Etudes massaliètes 3, 1992, p. 419-430.

Perrin, Decourt 2002 : PERRIN (F.), DECOURT (J.-C.). - L'aristocratie celte dans les sources littéraires. In : GUICHARD (V.), PERRIN (F.) dir. - L'aristocratie celte à la fin de l'Age du Fer (Ile s. av. J.-C., Ier s. ap. J.-C.). Actes de la table ronde des 10 et 11 juin 1999 (Glux-en-Glenne). Glux-enGlenne : Centre archéologique du Mont Beuvray, 2002, p. 337412 (Bibracte ; 5).

Petersen 1888 : PETERSEN (H.). -Vognfundene i Dejbjerg Proestegaardsmose ved Ringkjøbing 1881 og 1883 et bidrag til oplysning om den forromerske jernalder i Danmark. Kjøbenhavn, 1888.

Pič 1906 : PIC (J. L.). - Le Hradischt de Stradonitz en Bohême. Leipzig, 1906.

Piette 1989 : PIETTE (J.). - Notes sur le sanctuaire du Sute des Grèves à La Villeneuve-au-Châtelot (Aube). In : Pré-et Protohistoire de l'Aube. Vertus, 1989, p. 247-255.

Pinet 1972 : PINET (R.). - Saint-Romain-de-Jalionas, Gisement archéologique d'une tuilerie antique au lieudit "le Terreau". Evocations, 1972, p. 102-111.

Provost 1983 : PROVOST (M.). - Une anse de chaudron étrusque. Gallia 41, 1983, p. 209-215.

Py 1981 : PY (M.). - Recherches sur Nîmes préromaine. Paris, 1981 (Suppl. à Gallia 41).

Quilling 1903 : QUILLING (F.). - Die Nauheimer Funde der Hallstatt-und Latène-Periode in den Museen zu Frankfurt a. M. und Darmstadt. Frankfurt, 1903.

Quinn 1995 : QUINN (D.). - La Nécropole de la Croix du Rebout, Commune de Saint-Léger-sous-Beuvray, Saône-et-Loire). R. A. E. nº 46, 1995, p. 240-245.

Raddatz 1967 : RADDATZ (K.). - Das Wagengrab der jüngeren vorrömischen Eisenzeit von Husby, Kreis Flensburg. Offa-Bücher 20 ; Neumünster, 1967.

Raddatz 1969 : RADDATZ (K.). - Die Schatzfunde der iberischen Halbinsel vom Ende des dritten bis zur Mitte des ersten Jahrhunderts vor Chr. Geb. Untersuchungen zur hispanischen Toreutik. Madrider Forschungen 5 ; Berlin, 1969.

Raftery 1988 : RAFTERY (B.). - Hollow two-piece metal rings in La Tène Europe. Marburger Studien zur Vor-und Frühgeschichte 11 ; Marburg, 1988. 
Rasch 1994 : RASCH (M.). - The appearance of weapon graves in Scandinavia around the Birth of Christ Some interpretations in the light of weapon graves from Öland. In : CARNAP-BORNHEIM (C. von) dir. Beiträge zu römischer und barbarischer Bewaffnung in den ersten vier nachchristlichen Jahrhunderten. Akten des 2. Internationalen Kolloquiums Marburg 1994 ; Veröffentlichungen des Vorgeschichtlichen Seminars Marburg Sonderband 8 ; Lublin, Marburg, 1994, p. 223-231.

Rathgen 1924 : RATHGEN (F.). - Die Konservierung von Altertumsfunden, II. Teil, Metalle und Metallverbindungen. Berlin, Leipzig, 1924.

Raynard 1819 : RAYNARD (M.). - Recherche sur un mors de bride antique. In : Compte rendu des Travaux de la Société Royale d'Agriculture, Histoire Naturelle et Arts Utiles de Lyon. 1819, p. 192-197.

Rémy 1970 : REMY (B.). - Les limites de la cité des Allobroges. Cahiers d'Histoire, 15, p. 195-213.

Rémy 2000 : REMY (B.). - A propos du Rhône comme limite de la cité de Vienne au Haut Empire (en amont de Lyon). RAN., $\mathrm{n}^{\circ} 33,2000$ p. 55-61.

Rybovâ, Motyková 1983 : RYBOVA (A.), MOTYKOVA (K.). - Der Eisendepotfund der Latènezeit von Kolín. Pamatky archeologické 74, 1983, p. 96-174.

Rieckhoff 1998 : RIECKHOFF (S.). - Ein « keltisches Symposium ». Spatrepublikanisches Bronzegeschirr vom Mont Beuvray als wirtschaftlicher und gesellschaftlicher Faktor. In : Studien zur Archäologie der Kelten, Römer und Germanen in Mittel-und Westeuropa. Alfred Haffner zum 60. Geburtstag gewidmet. Internationale Archäologie Studia Honoria 4 ; Rahden/Westfalen, 1998, p. 489-517.

Riedel 1993/94 : RIEDEL (G.). - Fundchronik der Jahre 1993 und 1994/1 für den Altlandkreis Ingolstadt (Stadt Ingolstadt, Landkreise Eichstätt und Pfaffenhofen). Sammelblatt des Historischen Vereins Ingolstadt 102/103, 1993/94, p. 231-254.

Ritterling 1912 : RITTERLING (E.). - Das frührömische Lager bei Hofheim im Taunus. Nassauische Annalen 40, 1912, p. 1-416.

Rolley 1982 : ROLLEY (C.). - Un problème idéologique : est-ce que les Illyriens se lavaient les pieds ? Revue des Etudes Grecques 95, 1982, p. 24-25.

Rolley 1983 : ROLLEY (Cl.). - Les bronzes grecs. Fribourg, Paris, 1983.

Rolley 1987 : ROLLEY (Cl). - Deux notes de chronologie celtique. II. La situle de Waldalgesheim et la date du Style végétal continu. R. A. E., 38, 3-4, 1987, p. 416-419.

Rolley 1991 : ROLLEY (Cl.). - Bronzes en Messapie. In : I Messapi. Atti del $30^{\circ}$ convegno di studi sulla Magna Grecia (Taranto 1990). Tarente, 1991, p. 185-207.

Römer 2000 : Die Römer zwischen Alpen und Nordmeer. Zivilisatorisches Erbe einer europäischen Militärmacht. (Exposition Rosenheim). Schriftenreihe der Archaologischen Staatssammlung 1 ; München, 2000.

Ronke 1987 : RONKE (J.). - Magistratische Reprasentation im romischen Relief. Studien zu standes-und statusbezeichnenden Szenen. BAR International Series 370 ; Oxford, 1987.

Röring 1983 : RÖRING (Ch. W.). - Untersuchungen zu romischen Reisewagen. Koblenz, 1983.

Roualet et al. 1982 : ROUALET (P.), RAPIN (A.), FLUZIN (P.), URAN (L.). - Sépultures du Crayon, à Écury-leRepos (Marne). Mémoires de la Société d'agriculture, commerce, sciences et art du département de la Marne 97, 1982, p. 25-44.

Royet 1986 : ROYET (R.). - La villa de Saint-Romain-de-Jalionas et son environnement dans l'Antiquité. Mémoire de maîtrise, 2 vol., Université d'Aix-Marseille I, 1986.

Royet 2002 : ROYET (R.). - Un palais rural et son système domanial : Saint-Romain-de-Jalionas, Le Vernai (Isère). In : Les Allobroges, Gaulois et Romains du Rhône aux Alpes, Infolio éd. 2002, p. 82-85.

Royet, Verdin 2000 : ROYET (R.), VERDIN (F.). - Servanes à Mouriès ou les faubourgs de l'oppidum des Caisses de Saint Jean. In : LEVEAU (P.), SAQUET (J.-P.) dir. - Milieu et société dans la Vallée des Baux, Etudes présentées au colloque de Mouriès. Montpellier, 2000, (Suppl. à la R. A. N., ; 31). 
Royet, Faucher 2002 : ROYET (R.), FAUCHER (F.). - La villa de Sainte Colombe et le soit-disant vicus de Travers à La Balme les Grottes (Isère). Réinterprétation des données archéologiques. R. A. N., n 35, 2002, p. 259-271.

Roymans 1990 : ROYMANS (N.). - Tribal Societies in Northern Gaul. An anthropological perspective. Cingula 12 ; Amsterdam, 1990.

Rvbová, Motykovâ 1983 : RYBOVA (A.), MOT YKOVA (K.). - Der Eisendepotfund der Latènezeit von Kolín. Pamatky archeologické 74, 1983, p. 96-174.

Salzani 1983/84 : SALZANI (L.). - Contributi per la conoscenza della tarda età del Ferro nel Veronese. Atti e Mem. Accademia Agricoltura Scienze e Lettre Verona Ser. 6, 35, 1983/84, p. 353-381.

Salzani 1995 : SALZANI (L.). - La necropoli gallica di Valeggio sul Mincio. Documenti di Archeologia 5 ; Mantova, 1995.

Salzani 1998 : SALZANI (L.). - La necropoli gallica di Casalandri a Isola Rizza (Verona). Documenti di Archeologia 14, Mantova, 1998.

Sandoz 1989 : SANDOZ (G.). - Céramiques domestiques, fibules, monnaies et mobilier divers. In : GOUDINEAU (Chr.) dir. - Aux origines de Lyon. Lyon : Circonscription des Antiquités historiques, 1989, p. 67-76 (DARA ; 2).

Sassatelli 1987 : SASSATELLI (G.). - Un « nuovo » candelabra etrusco da Spina. Aspetti ellenizzanti nella cultura dell'Etruria padana. In : Celti ed Etruschi nell'Italia centro-settentrionale dal V secolo a.C. alla romanizzazione. Atti del Colloquio Internazionale (Bologna 12-14 aprile 1985). Imola, 1987, p. 61-87.

Scarpinelli 1979 : SCARPINELLI (D.). - Materiale protostorico del Compito. Studi Romagnoli 30, 1979, p. 357-379.

Schaaff 1985 : SCHAAFF (U.). - Studien zur keltischen Bewaffnung. Jahrbuch RGZM 32, 1985, p. 695-699.

Schaaff 1988 : SCHAAFF (U.). - Keltische Helme. In : Antike Helme. Sammlung Lipperlieide und andere Bestände des Antikenmusums Berlin. Mainz : Monographien RGZM 14, 1988, p. 293-317.

Schindler 1971 : SCHINDLER (R.). - Ein Kriegergrab mit Bronzehelm der Spätlatènezeit aus Trier-Olewig. Zum Problem des vorrömischen Trier. Trierer Zeitschrift 34, 1971, p. 43-82.

Schönberger 1952 : SCHÖNBERGER (H.). - Die Spätlatènezeit in der Wetterau. Saalburg-Jahrbuch 11, 1952, p. 21-130.

Schönfelder 2000a : SCHÖNFELDER (M.). - Das spätkeltische Wagengrab von Boé (Dép. Lot-et-Garonne). Studien zu Wagen und Wagengräbern der jüngeren Latènezeit. Thèse soutenue à Marburg, le 14 février 2000 ; http :// archiv.ub.uni-marburg.de/diss/z 2000/0093/.

Schönfelder 2000b : SCHÖNFELDER (M.). - Le mobilier métallique de la tombe à char tardo-celtique de Boé (Lot-et-Garonne). Aquitania 17, 2000, p. 59-81.

Schönfelder 2002 : SCHÖNFELDER (M.). - Das spätkeltische Wagengrab von Boé (dép. Lot-et-Garonne). Studien zu Wagen und Wagengräbern der jüngeren Latènezeit; avec résumé français. Mainz : Monographien RGZM 54, 2002.

Schulten 1929 : SCHULTEN (A.). - Die Loger bei Renieblas. Numantia. Die Ergebnisse der Ausgrabungen 19051912, vol. 4 ; München. 1929.

Schumacher 1911 : SCHUMACHER (K.). - Grabfund der sog. Mittel-La Tène-Zeit von Dühren (Bez.-A. Sinsheim, Grossh. Baden). Alterthümer unserer heidnischen Vorzeit 5 ; Mainz, 1911, p. 73-81, pl. 15.

Schumacher 1999 : SCHUMACHER (F.-J.). - Bewaffnung und Kampfesweise der Bevölkerung Nordgalliens in der Spätlatènezeit und frührömischen Zeit. Thèse Saarbrücken 1987 ; Saarbrücken, 1999.

Sehested 1878 : SEHESTED (F.). - Fortidsminder og Olsager fra Egnen om Broholm, 1878.

Sievers 1995 : SIEVERS (S.). - Die Waffen. In : REDDÉ (M.). - Fouilles et recherches nouvelles sur les travaux de César devant Alésia (1991-1994). Berichte der Römisch-Germanischen Kommission 76, 1995, p. 135-157. 
Simonnet, Quibqueton, Laborde 1983 : SIMMONET (L.), QUIBQUETON (A.), LABORDE (A.). - Aspects du gisement de La Tène de Marcilhac (commune de Saint-Paulien, HauteLoire). In : COLLIS (J.), DUVAL (A.), PÉRICHON (R.) (éd.). Le deuxième âge du fer en Auvergne et en Forez et ses relations avec les régions voisines. Université de Sheffield, Centre d'Eudes foréziennes, Saint Etienne, 1983, p. 97-105.

Spagnolo Garzoli 1999 : SPAGNOLO GARZOLI (G.) (dir.). - Conubia Gentium : la necropoli di Oleggio e la romanizzazione dei Vertamocori. Torino, 1999.

Stead 1967 : STEAD (I.-M.). - A La Tène III Burial at Welwyn Garden City. Archaeologia 101, 1967, 1-62.

Stümpel 1954 : STÜMPEL (B.). - Spätlatènekeramik in Rheinhessen. Eine Untersuchung zur Wangionenfrage. Thèse Mainz, non-publiée,1954.

Tarpin 2002 : TARPIN (M.) - Les pagi des Allobroges et l'organisation du territoire. In : Les Allobroges, Gaulois et Romains du Rhône aux Alpes. De l'indépendance à la période romaine (4e siècle av. J.-C. - 2e siècle apr. J.-C.). Infolio éd., 2002, p. 99-101.

Tassinari 1996 : TASSINARI (S.). - Bouillir, mijoter et cuire, porter et mesurer : le rôle de la vaisselle métallique dans les cuisines de Pompei. In : Les céramiques communes de Campanie et de Narbonnaise (Ier s. av. J.C. - IIe s. ap. J.C.). La vaisselle de cuisine et de table. Actes des Journées d'étude Naples 1994 ; Naples, 1996, p. 113-119.

Tendille 1988 : TENDILLE (C.). - Objets métalliques de la protohistoire au musée archéologique de Nîmes. Nîmes : Cahiers des musées et monuments de Nîmes 5, 1988.

Teodor 1980 : TEODOR (S.). - Das Werkzeugdepot von Lozna (Kr. Botosani). Dacia 24, 1980, p. 133-150.

Thierrin-Michael 1992 : THIERRIN-MICHAEL (G.). - Römische Weinamphoren : Mineralogische und chemische Untersuchungen zur Klärung ihrer Herkunft und Herstellungsweise. Freiburg : Universität Freiburg, Dissertation nr. 977, 1992.

Thill 1967 : THILL (G.). - Die Metallgegenstände aus vier spätlatènezeitlichen Brandgräbern bei Goeblingen-Nospelt. Hémecht 19, 1967, p. 87-98.

Thoma 1993 : THOMA (M.). - Das reich ausgestattete Brandgrab 1726 der Spätlatènezeit aus Wederath. Eine antiquarische, soziologische und historische Analyse. Thèse Kiel, publiée en microfiche, 1993.

Timpel 1980 : TIMPEL (W.). - Das altthüringische Wagengrab von Erfurt-Gispersleben. Altthüringen 17, 1980, p. 181-238.

Tizzoni 1981 : TIZZONI (M.). - La cultura tardo La Tène in Lombardia. Bergamo : Studi Archeologici 1, 1981.

Tizzoni 1982 : TIZZONI (M.). - I materiali della tarda etá del Ferro al civico museo di Lecco. Rassegna Stud. Civico Mus. Arch. Milano 29/30, 1982, p. 43-57.

Todorovic 1972 : TODOROVIC (J.). - Praistorijska Karaburma I. Nekropola mladteg gvozdenog doba. Diss, et Monogr. 8 ; Beograd, 1972.

Ulbert 1960 : ULBERT (G.). - Ein spätlatènezeitlicher Bronzebecher aus Manching, Ldkr. Ingolstadt. Bayerische Vorgeschichtsblätter 25, 1960, p. 69-75.

Ulbert 1984 : ULBERT (G.). - Cáceres el Viejo. Ein spätrepublikanisches Legionslager in Spanisch-Extremadura. Madrider Beiträge 11 ; Mainz, 1984.

Ulysse, Guillaume 1991 : ULYSSE (J.), GUILLAUME (M.). - Tombe à incinération d'un guerrier gaulois à Sigoyer (Hautes-Alpes). In : Archéologie dans les Hautes-Alpes. Gap, 1991, p. 217-221.

Unz, Deschler-Erb 1997 : UNZ (Ch.). DESCHLER-ERB (E.). - Katalog der Militaria aus Vindonissa. Militärische Funde, Pferdegeschirr und Jochteile. Veröffentlichungen der Gesellschaft Pro Vindonissa 14 ; Brugg, 1997.

Vaireaux 1996 : VAIREAUX (Fr.). - Saint-Vulbas. In : LE NEZET-CELESTIN (M.), VAIREAUX (Fr.), VICHERD (G.). - Izernore et Saint-Vulbas. Deux bourgades antiques de l'Ain. Cahiers René de Lucinge, n 31, Paris, 1996, p. $61-122$. 
Van der Wielen 1999 : VAN DER WIELEN (Y.). - Les monnaies des Allobroges. In : Monnayages Allobroges. Lausanne : Cahiers Romans de numismatique 6, 1999.

Van Endert 1991 : VAN ENDERT (D.). - Die Bronzefunde aus dem Oppidum von Manching. Kommentierter Katalog. Die Ausgrabungen in Manching 13 ; Stuttgart, 1991.

Vercingétorix 1994 : Vercingétorix et Alésia. Musée des Antiquités nationales Saint-Germain-en-Laye, Paris, 1994.

Verger 1996 : VERGER (S.). - Une tombe à char oubliée dans l'ancienne collection Poinchy de Richbourg. Mélanges de l'École Française Rome Antiquité 108/2, 1996, p. 641-691.

Verger. Guillaumet 1988 : VERGER (S.), GUILLAUMET (J.-P.). - Les tumulus de Saint-Romain-de-Jalionas (Isère). Premières observations. In : Les princes celtes et la Méditerranée. Paris : Rencontres de l'Ecole du Louvre. La Documentation Française, 1988, p. 231-240.

Versnel 1970 : VERSNEL (H. S.). - Triumphus. An Inquiry into the origin, development and meaning of the roman triumph. Leiden, 1970.

Vidal 1991 : VIDAL (M.). - La vaisselle tardo-républicaine en Gaule du Sud-Ouest. In : FEUGERE (M.), ROLLEY (CL), éd., - La vaisselle tardo-républicaine en bronze. Actes de la table-ronde Lattes 1990. Dijon : Université de Bourgogne, Centre des recherches sur les techniques gréco-romaines 13, 1991, p. 169-191.

Vierrädrige Wagen 1987 : Vierrädrige Wagen der Hallstattzeit. Untersuchungen zu Geschichte und Technik. Mainz : Monographien RGZM 12, 1987.

Visy 1993 : VISY (Z.). - Wagen und Wagenteile. In : KÜNZL (E.). éd., - Die Alamannenbeute aus dem Rhein bei Neupotz. Plünderungsgut aus dem romischen Gallien. Mainz : Monographien RGZM 34, 1, 1993, p. 257-327.

Völling 1992 : VÖLLING (Th.). - Dreikreisplattensporen - Bemerkungen zu einem Spornfund aus Hopferstadt, Ldkr. Würzburg. Archäologisches Korrespondenzblatt 22, 1992, p. 393-402.

Vouga 1885 : VOUGA (E.). - Les Helvètes à La Tène. Neuchâtel. 1885.

Wamser 1982 : WAMSER (L.). - Spätkeltische Einzelfunde aus der Uferzone eines Altsees am Fuße des Schwanbergs in der Gemarkung Großlangheim, Landkreis Kitzingen, Unterfranken. Das archäologische Jahr in Bayern 1982, p. 83-86.

Wamser 1984 : WAMSER (L.). - Eine thüringischfränkische Adels-und Gefolgschaftsgrablege des 6./7. Jahrhunderts bei Zeuzleben, ein Vorbericht. Mainfränkisches Jahrbuch für Geschichte und Kunst 36, 1984, p. $1-22$.

Wegewitz 1982 : WEGEWITZ (W.). - Ein Bronzeeimer der Spätlatènezeit vom Greifensee. Helvetia Archaeologica 49, 1982, 39-48.

Wehrberger, Wieland 1999 : WEHRBERGER (K), WIELAND (G.). - Ein weiteres Knollenknaufschwert und eine Aylesford-Pfanne aus der Donau bei Ulm. Überlegungen zu spätkeltischen und frührömischen Gewässerfunden von der oberen Donau. Archäologisches Korrespondenzblatt 29, 1999, 237-256.

Werner 1953 : WERNER (J.). - Keltisches Pferdegeschirr der Spätlatènezeit. Saalburg-Jahrbuch 12, 1953, p. 42-52.

Wieland 1996 : WIELAND (G.). - Die Spätlatènezeit in Württemberg. Forschungen zur jüngeren Latènekultur zwischen Schwarzwald und Nordlinger Ries. Forschungen und Berichte zur Vor-und Frühgeschichte in BadenWürttemberg vol. 63 ; Stuttgart, 1996.

Wielowiejski 1985 : WIELOWIEJSK1 (J.). - Die spätkeltischen und romischen Bronzegefäße in Polen. Berichte der Römisch-Germanischen Kommission 66, 1985, p. 123-320.

\section{RAPPORTS DE FOUILLES}

Convert 1994 : CONVERT (R. ). - Contribution à la carte archéologique du Nord Dauphiné. Rapport multigraphié, S.R.A. Rhône-Alpes, 1994. 
Fichtl, Adam 2000 : FICHTL (St.), ADAM (A.-M.).- L'oppidum médiomatrique du Fossé des Pandours au Col de Saverne (Bas-Rhin). Université Marc Bloch - UPR SA 7044 : Rapport intermédiaire 2000.

Guillaumet, Porte 1986 - GUILLAUMET (J.-R), PORTE (P.). - Redécouverte d'une tombe à char à Verna/SaintRomain de Jalionas. Xe colloque de TAFEAF, Yenne-Chambéry, mai 1986, dactylographié.

Hénon 1999 : HENON (P.). - Sondages archéologiques effectués dans la parcelle Verdolini à St-Romain-deJalionas (Isère) - Parcelles 43, 48. et 49, D.F.S. multigraphié, S.R.A. Rhône-Alpes, 1999.

Hettiger 1990 : HETTIGER (S.). - Tumulus et structures funéraires des âges du Fer dans le Nord-Isère (communes de Saint-Romain-de-Jalionas, Leyrieu, Verna). Rapport de fouille multigraphié, D.A.H. RhôneAlpes, 1990.

Porte 1992 : PORTE (P.) (dir.). — Le site archéologique de Larina de l'Antiquité Tardive au haut Moyen Age (Hières-sur Amby Isère). Rapport de fouille triennal 1989-1992, multigraphié, 2 vol., D.A.H. Rhône Alpes, 1992.

Rebillart 1971 : REBILLART (L). - Rapport de prospection aérienne 1971. Rapport d'activité multigraphié. D.A.P. Rhône-Alpes, 1971.

Revellin 1974 : REVELLIN (Ch.). - Le tumulus du Peillard à Saint-Romain-de-Jalionas (Isère). Rapport de fouille multigraphié, D.A.P. Rhône-Alpes, 1974.

Rieckhoff, Fleischer 2001 : RIECKHOFF (S.), FLEISCHER (F.). - Reconnaissance de l'angle nord-ouest de l'îlot dit « des grandes forges ». Rapport annuel d'activité 2001 du Centre archéologique européen du Mont Beuvray ; Glux-en-Glenne 2001, p. 91-98.

Royet et al. 2001 : ROYET (R.) BERGER (J.-Fr.), ROYET (E.).- Le Vernai à Saint-Romain-de-Jalionas (Isère). Rapport de synthèse 2001. DFS multigraphié, 6 vol, SRA Rhône-Alpes.

Verger, Guillaumet 1987 : VERGER (S.), GUILLAUMET (J.-P.). - Rites funéraires à l'âge du Fer dans le NordIsère, Bilan 1987. Rapport de fouille multigraphié., DAH Rhône-Alpes.

Vital, Chastel, De Klijn 1986 : VITAL (J.), CHASTEL (J.), DE KLIJN (J.). - Le site protohistorique et galloromain du Pré de la Cour à Montagnieu. Rapport de fouille multigraphié, 2 vol., DAH Rhône-Alpes, 1986. 


\section{Crédits illustrations}

\section{Clichés photographiques}

fig. 1 : Robert Royet.

fig. 2 à 6,52 et 1 (annexe 1): Collection privée.

fig. 7 : J.-R Guillaumet.

fig. 8 : Bibliothèque municipale de Lyon (Rhône).

fig. 12 et cliché de couverture : Solenne Paul, (Musée Dauphinois, Isère).

fig. 14, 23b et c, 26a, 38, 44, 51, 66. 67, 71, 76, 79, 90 : A. Maillier (CAE du Mont Beuvray, Bibracte).

Les clichés des monnaies de l'annexe ont été effectués par P. Plattier.

Quatrième de couverture : Académie des Sciences Belles-Lettres et Arts de Lyon

(Ms 104).

\section{Dessins}

La grande majorité des dessins ont été effectués par M.-N. Baudrand sauf mention contraire :

fig. 9, 10, 11 : R. Royet.

fig. 89 : V. Guichard.

fig. 59 : RGZM. 